\title{
ILLUMINATING THE STRUCTURE OF BORIDES THROUGH $X$-RAY ABSORPTION SPECTROSCOPY
}

Sebastiaan P. Huber

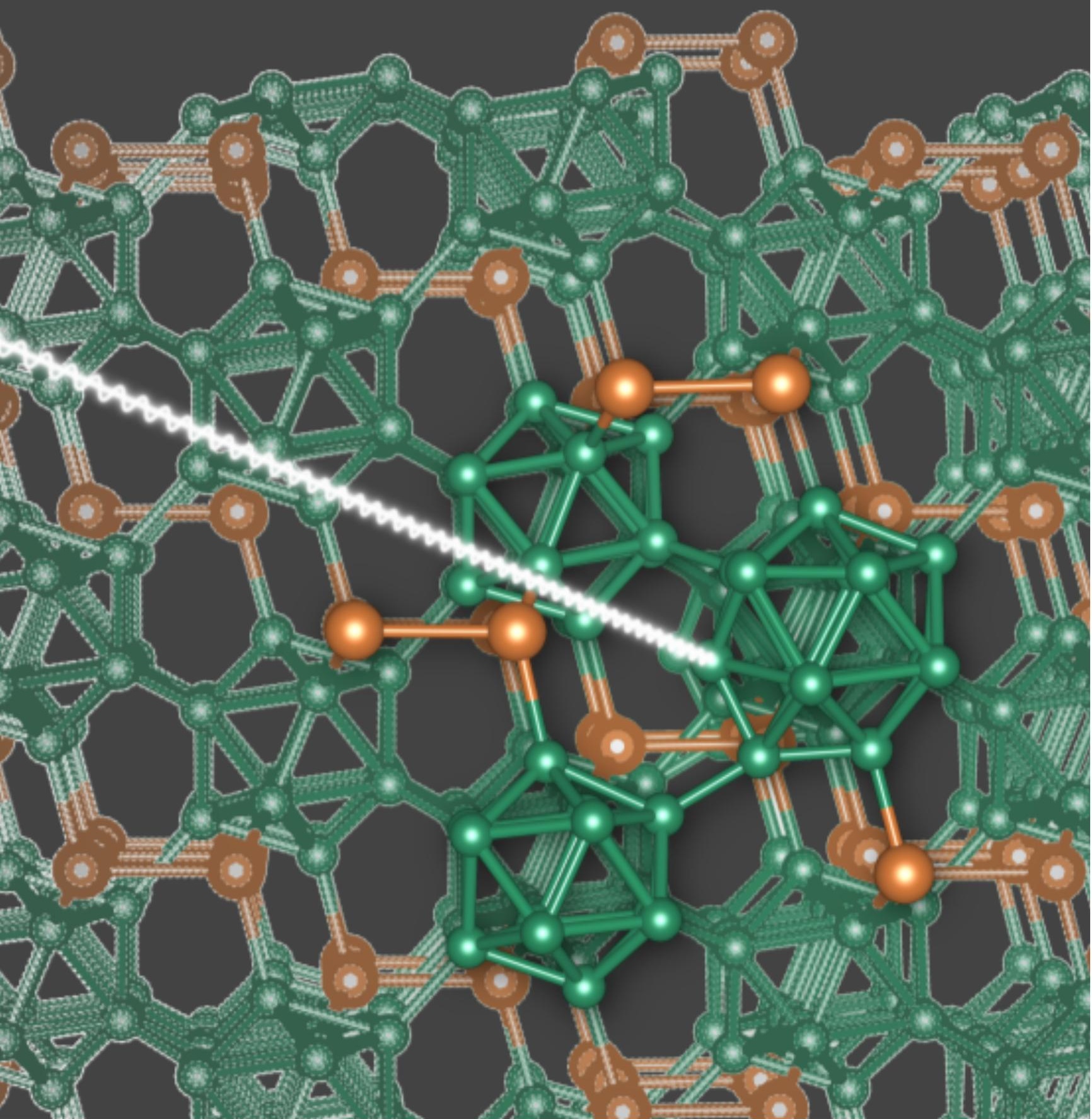





\title{
ILLUMINATING THE STRUCTURE OF BORIDES THROUGH X-RAY ABSORPTION SPECTROSCOPY
}

\author{
PROEFSCHRIFT \\ ter verkrijging van \\ de graad van doctor aan de Universiteit Twente, \\ op gezag van de rector magnificus \\ prof. dr. H. Brinksma, \\ volgens besluit van het College voor Promoties, \\ in het openbaar te verdedigen \\ op donderdag 20 oktober 2016 des middags te 13.00 uur \\ door

\section{Sebastiaan Philippe Huber}

geboren op 17 oktober 1986

te Voorburg 
Promotor: $\quad$ Prof. dr. F. Bijkerk

Co-promotor: Dr. ir. R. W. E. van de Kruijs

Committee: Prof. dr. ir. J. W. M. Hilgenkamp (voorzitter/secretaris)

Dr. D. Prendergast

Prof. dr. T. T. M. Palstra

Prof. dr. B. J. Thijsse

Prof. dr. P. J. Kelly

Prof. dr. W. J. Briels

Dit proefschrift is goedgekeurd door de promotor en co-promotor

Copyright: 2016 (C) Sebastiaan P. Huber

ISBN: $\quad 978-90-365-4182-4$

DOI: $\quad 10.3990 / 1.9789036541824$ 


\section{ILLUMINATING THE STRUCTURE OF BORIDES THROUGH X-RAY ABSORPTION SPECTROSCOPY}

Sebastiaan Philippe Huber

September 26, 2016 
This work is part of the research programme of FOM (Stichting voor Fundamenteel Onderzoek der Materie), which is part of NWO (Nederlandse Organisatie voor Wetenschappelijk Onderzoek) and is supported by NanoNextNL, a micro and nanotechnology programme of the Dutch Government and 130 partners. The author also acknowledges the support of the Industrial Focus Group XUV Optics enabled by the University of Twente, the MESA + Institute for Nanotechnology, the Province of Overijssel, ASML, Carl Zeiss SMT AG, PANalytical, DEMCON, SolMateS, as well as FOM and NWO through the Industrial Partnership Programme CP3E. 


\section{CONTENTS}

Contents $\quad$ v

List of Publications $\quad$ ix

1 General introduction $\quad 1$

1.1 The fifth element . . . . . . . . . . . . . . . . . 3

1.1.1 Hexagonal boron nitride . . . . . . . . . . . . . . . 3

1.1.2 Icosahedral borides . . . . . . . . . . . . . . . . . 4

1.1.3 Cubic boron phosphide . . . . . . . . .... 5

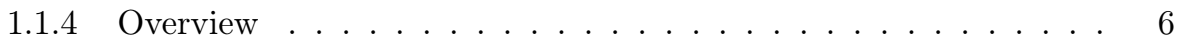

1.2 X-ray absorption near-edge spectroscopy . . . . . . . . . . . . . 6

1.3 Density functional theory . . . . . . . . . . . . . . . . . . . 9 9

1.4 X-ray absorption spectroscopy from first principles . . . . . . . . . . . 10

1.4.1 Fermi's golden rule . . . . . . . . . . . . . . . . . . . 10

1.4.2 The pseudopotential plane wave implementation . . . . . . . . . 11

1.4.3 Excited electron core-hole approach . . . . . . . . . . . . . 12

1.5 Experimental x-ray absorption spectroscopy . . . . . . . . . . . . . . 12

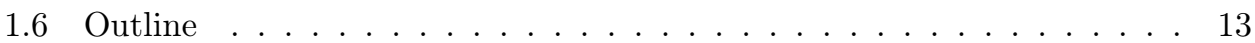

$\begin{array}{ll}\text { References } & 15\end{array}$

2 Computational method $\quad 19$

2.1 Crystal structure definition . . . . . . . . . . . . . . . . 21

2.2 Ab-initio molecular dynamics . . . . . . . . . . . . . . . . . . 22

2.3 Calculating the electronic structure . . . . . . . . . . . . . 23

2.4 Optimized basis sets . . . . . . . . . . . . . . . . . 24

2.5 Post-processing of computed absorption spectrum . . . . . . . . . . . . 24

$\begin{array}{ll}\text { References } & 27\end{array}$ 
3 Oxygen-stabilized triangular defects in hexagonal boron nitride 29

3.1 Introduction . . . . . . . . . . . . . . . . 31

3.2 Experimental details . . . . . . . . . . . . . . . . . . . . . . . . . . . . . . . . . . . . . . . . . 32

3.3 Computational method . . . . . . . . . . . . . . . . . 32

3.3.1 Structural relaxation and molecular dynamics . . . . . . . . . . 32

3.3 .2 Formation energies . . . . . . . . . . . . . . . 33

3.3 .3 X-ray absorption spectroscopy . . . . . . . . . . . . 33

3.4 Results . . . . . . . . . . . . . . . . . . . 34

3.4.1 Structure of triangular voids . . . . . . . . . . . . . . 34

3.4.2 Triangular defect formation energy . . . . . . . . . . . . . . 37

3.4.3 X-ray absorption spectroscopy of hexagonal boron nitride . . . . 39

3.4.4 Oxygen defects form triangular voids . . . . . . . . . . . . 41

3.4.5 X-ray absorption spectroscopy of oxygen defects . . . . . . . . 41

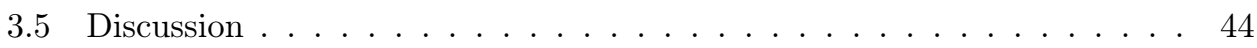

3.6 Conclusions . . . . . . . . . . . . . . . . . . 45

$\begin{array}{ll}\text { References } & 47\end{array}$

4 Self-healing in $\mathrm{B}_{12} \mathrm{P}_{2}$ through mediated defect recombination $\quad 49$

4.1 Introduction . . . . . . . . . . . . . . . . . . 51

4.2 Computational Method . . . . . . . . . . . . . . . 53

4.2.1 Structural relaxation . . . . . . . . . . . . . . . . . . . 53

4.2 .2 Nudged elastic band . . . . . . . . . . . . . . . . . . . . 53

4.2 .3 Molecular dynamics . . . . . . . . . . . . . . . . . . . 54

4.2 .4 Charged defects . . . . . . . . . . . . . . . 54

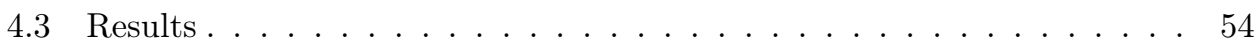

4.3.1 Crystal structure . . . . . . . . . . . . . . 54

4.3.2 Interstitial and vacancy defects . . . . . . . . . . . . . 56

4.3.3 Frenkel defect recombination pathways . . . . . . . . . . . . 59

4.3 .4 Vacancy diffusion . . . . . . . . . . . . . . . . . . . 59

4.3 .5 Interstitial diffusion . . . . . . . . . . . . . . . . . 62

4.3.6 Frenkel defect recombination . . . . . . . . . . . . . . . 66

4.3.7 Frenkel recombination dynamics . . . . . . . . . . . . . . . . . 72

4.3.8 Frenkel defect charge localization . . . . . . . . . . . . . . 78

4.3 .9 Charged Frenkel defects . . . . . . . . . . . . . . . . . 80

4.4 Conclusions . . . . . . . . . . . . . . . . . . . 82

$\begin{array}{lr}\text { References } & 84\end{array}$

5 Detection of defect populations in superhard semiconductor boron subphosphide $\mathbf{B}_{12} \mathbf{P}_{2}$ through x-ray absorption spectroscopy $\quad \mathbf{8 7}$

5.1 Introduction . . . . . . . . . . . . . . . . . . . . . 89

5.2 Experimental details . . . . . . . . . . . . . . . . . . . 90 
5.3 Computational method . . . . . . . . . . . . . . . . . . . . 90

5.3.1 Structural relaxations . . . . . . . . . . . . . . . . . 90

5.3 .2 Formation energies . . . . . . . . . . . . . . . . . . . . . . 91

5.3 .3 X-ray absorption spectroscopy . . . . . . . . . . . . . 91

5.4 Experimental results . . . . . . . . . . . . . . . . . . . . . . . . . . . . . . . . . . . . . . . .

5.4 .1 Sample deposition . . . . . . . . . . . . . . . . 92

5.4 .2 X-ray absorption spectroscopy . . . . . . . . . . . . . . . 93

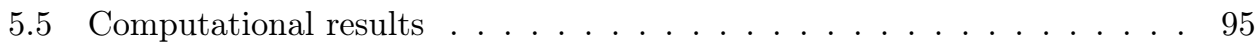

5.5 .1 Crystal structure . . . . . . . . . . . . . . . . 95

5.5.2 Formation energies of point defects . . . . . . . . . . . . . 95

5.5.3 Density of states . . . . . . . . . . . . . . . . . . . . . 97

5.5.4 Simulated x-ray absorption spectroscopy . . . . . . . . . . . . . . 98

5.5.5 Defect states and spectral alignment . . . . . . . . . . . . . 106

5.5.6 Point defect x-ray absorption spectroscopy . . . . . . . . . . 110

5.6 Conclusions . . . . . . . . . . . . . . . . . . . . . . . . . . . . . 113

References

6 Determining crystal phase purity in cubic boron phosphide through $\begin{array}{lr}\text { x-ray absorption spectroscopy } & 117\end{array}$

6.1 Introduction . . . . . . . . . . . . . . . . . . . . . 119

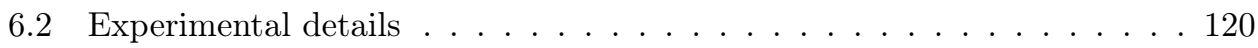

6.2.1 Cubic boron phosphide sample deposition . . . . . . . . . . . 120

6.2.2 Boron and boron nitride sample deposition . . . . . . . . . . . . 120

6.2.3 Total electron yield x-ray absorption spectroscopy . . . . . . . . 121

6.3 Computational method . . . . . . . . . . . . . . . . 121

6.3.1 X-ray absorption spectroscopy . . . . . . . . . . . . . 121

6.3.2 Structural optimizations . . . . . . . . . . . . . . . . . . . . 122

6.3.3 Crystal cell definitions and molecular dynamics . . . . . . . . . . 122

6.3.4 Simulation of amorphous structures . . . . . . . . . . . . 123

6.4 Experimental results . . . . . . . . . . . . . . . . . . . . . 123

6.4.1 X-ray absorption spectroscopy . . . . . . . . . . . . 123

6.5 Computational results . . . . . . . . . . . . . . . . . . 125

6.5.1 X-ray absorption spectroscopy of point defects . . . . . . . . 126

6.5.2 X-ray absorption spectroscopy of different crystal phases . . . . . 128

6.5.3 X-ray absorption spectroscopy of amorphous phases . . . . . . . 132

6.5.4 Formation enthalpies of boron compounds . . . . . . . . . . . . . 139

6.5.5 X-ray absorption spectroscopy of amorphous boron . . . . . . . . 140

6.6 Conclusions . . . . . . . . . . . . . . . . . . 142

$\begin{array}{lr}\text { References } & 144\end{array}$

$\begin{array}{llr}7 & \text { Valorization } & 147\end{array}$ 
viii

7.1 Goals and specifications . . . . . . . . . . . . . . . . . . . . . . . . . . . . . . . . . .

7.2 Global architecture and design . . . . . . . . . . . . . . . . . 149

7.2.1 Decoupling computation and data presentation . . . . . . . . 150

7.2.2 Communication protocol . . . . . . . . . . . . 151

7.3 Conclusions . . . . . . . . . . . . . . . . . . . 152

$\begin{array}{ll}\text { Bibliography } & 155\end{array}$

$\begin{array}{ll}\text { Acknowledgments } & 161\end{array}$

$\begin{array}{ll}\text { Nederlandse samenvatting } & 164\end{array}$ 


\section{LIST OF PUBLICATIONS}

The work presented in this thesis is based on the following publications

[1] S. P. Huber, E. Gullikson, R. W. E. van de Kruijs, F. Bijkerk, and D. Prendergast, "Oxygen-stabilized triangular defects in hexagonal boron nitride", Phys. Rev. B 92 (2015) 10.1103/physrevb.92.245310.

[2] S. P. Huber, E. Gullikson, J. Meyer-Ilse, C. D. Frye, J. H. Edgar, R. W. E. van de Kruijs, F. Bijkerk, and D. Prendergast, "Self-healing in $\mathrm{B}_{12} \mathrm{P}_{2}$ through mediated defect recombination", Submitted to Chemistry of Materials.

[3] S. P. Huber, E. Gullikson, C. D. Frye, J. H. Edgar, R. W. E. van de Kruijs, F. Bijkerk, and D. Prendergast,

"Detection of defect populations in superhard semiconductor boron subphosphide $\mathrm{B}_{12} \mathrm{P}_{2}$ through x-ray absorption spectroscopy", Submitted to Chemistry of Materials.

[4] S. P. Huber, V. V. Medvedev, E. Gullikson, B. Padavala, J. H. Edgar, R. W. E. van de Kruijs, F. Bijkerk, and D. Prendergast, "Determining crystal phase purity in $c$-BP through x-ray absorption spectroscopy", Submitted to Phys. Rev. B.

[5] S. P. Huber, R. W. E. van de Kruijs, A. E. Yakshin, E. Zoethout, K.-J. Boller, and F. Bijkerk,

"Subwavelength single layer absorption resonance antireflection coatings", Opt. Express 22, 490 (2014).

The latter has been completed during this work but is not described in this thesis 


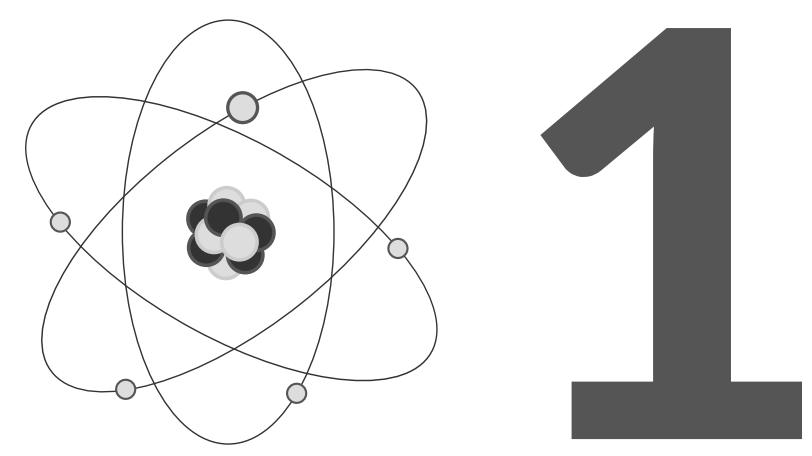

GENERAL INTRODUCTION 


\section{ABSTRACT}

This introductory chapter serves to set the stage for the research that will be presented in this thesis. It will answer the question why boron and its compounds are of particular interest and discuss the applications in which they can be used. The choice for x-ray absorption spectroscopy, as the main method of investigating the structure of borides on an atomic scale, will also be motivated. Finally, fundamental theoretical concepts will be introduced that are vital to the modeling of x-ray absorption spectroscopy as performed in this work. 


\subsection{THE FIFTH ELEMENT}

Boron, the fifth element in the periodic table of elements, forms the backbone of the work that will be presented in this thesis. As the lightest constituent of the group III elements, it is often classified as a metalloid, which is a broad term used to describe elements that are in between metals and non-metals in terms of the electrical properties of the compounds they tend to form. Despite its simple electronic configuration of two core electrons and three valence electrons, it is an extremely chemically versatile element, that will form compounds with electronic structures ranging from semimetals, through semiconductors all the way to large band gap insulators. The same electronic configuration also forms the foundation for the rich variety of crystal structures found in borides. From a simple hexagonal structure, as in the case of hexagonal boron nitride, to a complex network of boron icosahedra (a polyhedron with 12 vertices and 20 faces) and its derivatives in $\beta$-rhombohedral boron, a complex rhombohedral allotrope of boron, whose exact structure, with its partially occupied sites, is still not fully elucidated[6]. This wide range of structural and electronic properties, found in the different boron allotropes and boron containing compounds, have caused boron to be used in a broad spectrum of applications.

\subsubsection{HEXAGONAL BORON NITRIDE}

In the wake of the fame and glory of atomically thin layers of graphite, better known as graphene, whose discovery[7] was awarded the Nobel prize in physics in 2010, hexagonal boron nitride $h$-BN, being a direct structural analog, has seen a wealth of research. Many studies of its properties and applications have been published since the early 2000's, making it perhaps the most well-known and thoroughly studied boron containing material. Both graphite and $h$-BN share interesting material properties such as a high thermal conductivity[8,9] and chemical stability[10]. From an electronic perspective, however, they could not be more different, as graphite is a conductor[7] and $h$-BN is a wide gap insulator[11]. Ever since the first successful synthesis of monolayer hexagonal boron nitride $g$-BN[12], it has been suggested to be used in conjunction with graphene in novel nanoscale electronics. Their high structural commensurability yet contrasting electronic properties, might prove ideal to create atomically thin electronical components[13].

These types of applications typically require materials with a high degree of crystallinity, since structural defects often degrade the desirable material properties. However, many experimental studies that have grown $h$-BN, have reported the observation of triangular voids in synthesized samples, as shown in Fig. 3.1, and the nature of these defects is not yet fully known. In Chapter 3, the origin and character of these triangular shaped defects will be discussed and it will be shown that oxygen plays an important role in the formation of these defects. The newly gained knowledge will prove valuable 

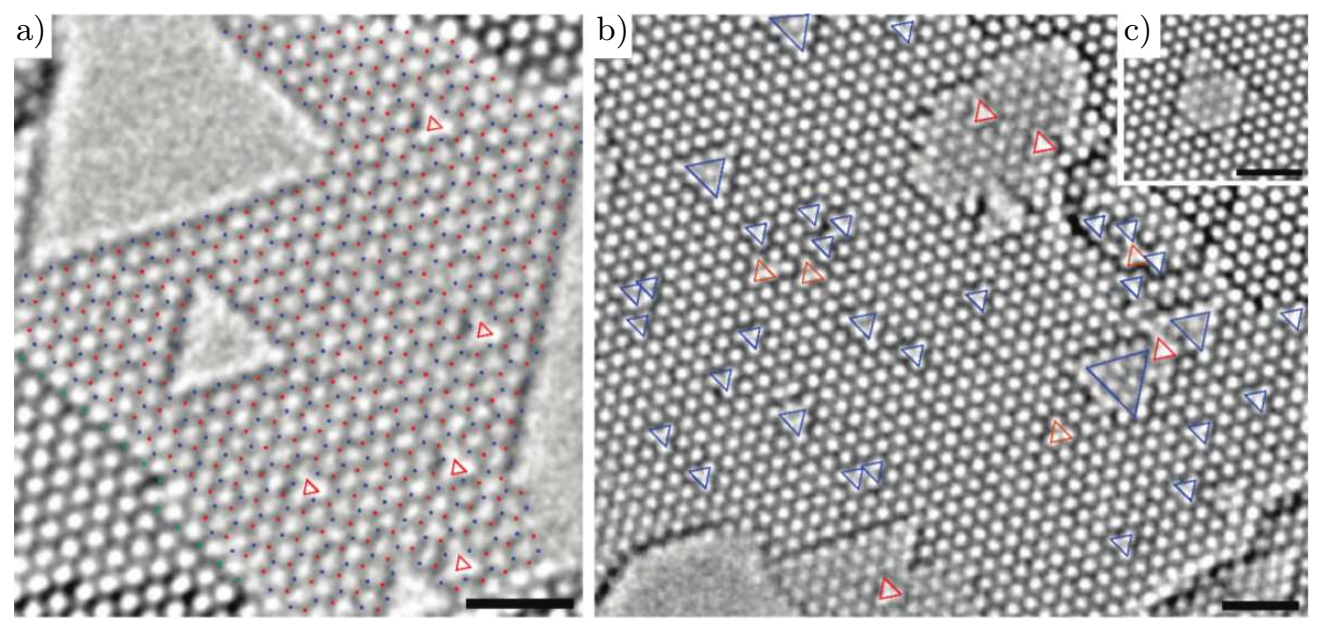

Figure 1.1: Transmission electron microscopy recording of a $h$-BN membrane with the characteristic triangular voids indicated by the red and blue triangles[14].

in future studies that aim to control the abundance of defects in $h$-BN, which will bring its application in next-generation electronics closer to reality.

\subsubsection{ICOSAHEDRAL BORIDES}

In stark contrast with the simple hexagonal crystal lattice of boron nitride, the allotropes of elemental boron are significantly more complicated. As a direct result of its electronic configuration, having three valence electrons available to form bonds, boron atoms tend to cluster in the shape of a regular icosahedron and these boron icosahedra, each containing 12 boron atoms, form the fundamental building blocks for many boron rich solids. The simplest boron allotrope, $\alpha$-rhombohedral, is defined by a rhombohedral arrangement of boron icosahedra and was long thought to be the most stable form of boron, although recent research, has shown that $\beta$-rhombohedral boron may in fact be more energetically favorable[6]. The $\alpha$-rhombohedral crystal structure is also known to form the basis of many other boron-rich solids such as $\mathrm{B}_{12} \mathrm{P}_{2}, \mathrm{~B}_{12} \mathrm{As}_{2}$, $\mathrm{B}_{12} \mathrm{O}_{2}, \mathrm{~B}_{13} \mathrm{C}_{2}$ and $\mathrm{B}_{4} \mathrm{C}$. These icosahedral borides share many interesting structural properties such as a high melting temperature, chemical inertness and a high neutron capture cross-section. The two pnictide variations $\mathrm{B}_{12} \mathrm{P}_{2}$ and $\mathrm{B}_{12} \mathrm{As}_{2}$, pnictogens being elements from group 15 of the periodic table, have also been hypothesized to exhibit a form of "self-healing"[15]. Even after prolonged exposure to highly energetic particles, these materials showed little to no structural damage [see Fig. 1.2], despite the fact that under the radiative conditions, each boron atom would have had to been displaced at least several times. The hypothesis is that these materials have the ability to automatically restore structural damage, created by the displacement of atoms. 
Before

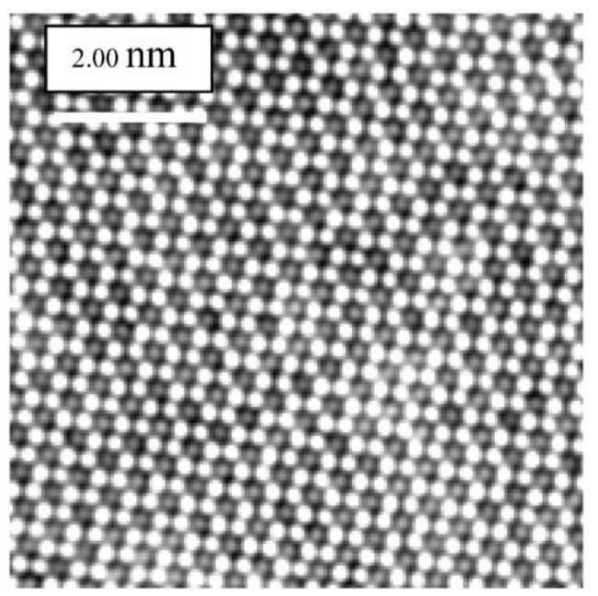

After

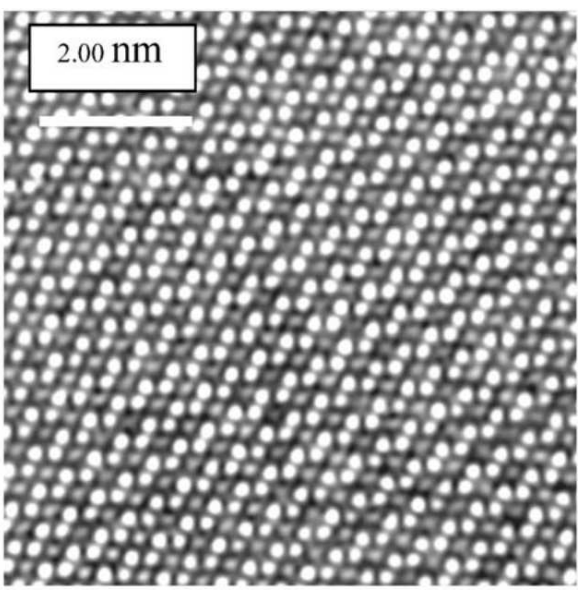

Figure 1.2: Transmission electron microscopy recordings of a $\mathrm{B}_{12} \mathrm{P}_{2}$ sample, before and after intense irradiation by highly energetic electrons[16]. In stark contrast with other borides, such as the diboride $\mathrm{TiB}_{2}$ and the octahedral borides $\mathrm{LaB}_{6}$ and $\mathrm{CaB}_{6}$, which were heavily damaged under similar conditions, $\mathrm{B}_{12} \mathrm{P}_{2}$ seemed almost undamaged by the radiation exposure.

The high radiation hardness, as a result of this "self-healing" mechanism, makes these materials interesting for a variety of applications such as beta-voltaic devices[16] and, combined with the relatively high neutron scattering cross-section of boron, neutron detectors[17, 18]. However, the real mechanism that underlies the intriguing damage restoring property is not yet understood and in order to maximize the potential of the radiation resistant borides in these applications, a better fundamental knowledge of the "self-healing" mechanism is required. In Chapter 4, the restorative qualities of $\mathrm{B}_{12} \mathrm{P}_{2}$ will be studied through calculations from first-principles, which will reveal the origins of the "self-healing" phenomenon. In Chapter 5, first steps are made to verify the theoretical predictions, made in the preceding chapter, through the study of x-ray absorption spectrosopy of various point defects. These spectroscopic defect "fingerprints" could be used in future experiments that will monitor the creation and recombination of defects through x-ray absorption spectroscopy in real time.

\subsubsection{CUBIC BORON PHOSPHIDE}

Borides have also found their way in several optical applications, for example they have been proposed as candidates for use in multilayer optics in next generation photolithography tools. The optical properties of borides around $188 \mathrm{eV}$, shaped primarily by the presence of the absorption $K$-edge, are used to create highly reflective multilayer optics out of alternating thin layers of lanthanum and a boride, targeted to operate at a wavelength around $6.5 \mathrm{~nm}[19-21]$. A more recent proposal considered the use of cubic 
boron phosphide $c$-BP to create multilayer mirrors that would operate near the absorption $K$-edge of $\mathrm{P}$ at $130 \mathrm{eV}$, which would allow for optics that operate efficiently at wavelengths near $10 \mathrm{~nm}[22]$. This enables an additional choice of operation wavelength, which increases the flexibility of the wavelength selection process in the development of next-generation photolithography. Various methods have been developed to grow cubic boron phosphide[23, 24], which now require methods to analyze the resulting crystal structures. Changes in the structure of $c$-BP may affect the optical properties of the material near the absorption edges, which will affect the optical properties of its applied optics and therefore will be of great relevance. In Chapter 6 , the quality and structure of synthesized cubic boron phosphide crystals will be studied, the results of which can be used to improve existing synthesis procedures and will show how optical properties can be affected by the structural properties of the material.

\subsubsection{OVERVIEW}

This short overview highlights the versatility of the boron element and the wide range of material properties its solid state compounds possess, as well as the broad spectrum of applications they can be used for as a result. Additionally, it briefly discussed where the current knowledge of the structure of borides is still lacking and how an improved understanding may benefit the use of borides in various applications. To enable further development of borides and its applications, one has to fully unravel and understand the origins of its desirable properties, and therewith the material's crystal and electronic structure on an atomic level, in which they are founded. An experimental technique that is exceptionally sensitive to exactly those qualities, the local chemical and structural environment of an atom, is x-ray absorption near-edge spectroscopy. Compared to other techniques such as x-ray photo-electron spectroscopy (XPS) for example, it has the advantage that it does not only provide information through the binding energy of the core electron, but it also maps the density of states near the conduction band, which can provide a wealth of information. The local character of the technique also ensures that information about atomic coordination can be determined even in the absence of long range order, which would not be possible with diffractive methods such as x-ray diffraction (XRD). The work presented in this thesis is centered around experimental x-ray absorption spectroscopy of various borides and the analysis thereof through modeling the spectroscopy from first principles. The results provide new insights in the structural and chemical properties of these borides, that improve the understanding of their functionality in their respective applications.

\subsection{X-RAY ABSORPTION NEAR-EDGE SPECTROSCOPY}

X-ray absorption near-edge spectroscopy (XANES), also referred to as near-edge $\mathrm{x}$ ray absorption fine structure (NEXAFS) spectroscopy, is a spectroscopic method that 
studies the characteristic absorption of photons through excitation of core-level electrons, as a function of the photon energy. The process is visualized schematically in four steps in Fig. 1.3.

An incident photon, given enough energy to overcome the binding energy of the core electron, can excite the electron into an unoccupied orbital, leaving behind a core hole in that atomic core level, see Fig. 1.3(b). These electron binding energies range from several tens of $\mathrm{eV}$ for the core levels of small elements and the semi-core levels of heavier elements, all the way to tens of thousands of $\mathrm{eV}$ for the core levels of the heaviest elements[25]. The excited electron-hole pair can decay through either an Auger process [see Fig. $1.3(\mathrm{c})$ ], where an electron fills the core-hole and a secondary electron is ejected from the atom, or a fluorescence process [see Fig. 1.3(d)], in which the electron-hole recombination is paired with the emission of a fluorescent photon. In typical XANES experiments, it is the resultant electrons and photons from these two decay channels that are collected, referred to as total electron yield (TEY) and fluorescence yield (FLY), respectively, and their spectrum is a direct measure of the photon absorption.

Due to the direct dependence of the core electron excitation process on the binding energy, which is core level specific, XANES has an extremely high element specificity. As a result, XANES enables the extraction of element specific information of atomic minority concentrations, even in the presence of large background signals. The nomenclature for specific absorption edges is based on the core level that is involved in the excitation and for the $K$ - and $L_{2,3}$-edges discussed in this work, they correspond to excitations of $1 s$ and $2 p$ electrons, respectively. In addition to the element specificity of XANES, the features of the absorption spectrum directly after the absorption edge, also contain a lot of information about the local chemistry and structure of the excited species. The probability of the excitation of a core electron by a photon into a specific unoccupied orbital, is not just dependent on the binding energy of the core level, but also on the character of the electronic state into which the electron is excited. The character of these unoccupied states is strongly influenced by the local atomic structure and chemistry in which the excited atom finds itself. As a result the characteristic x-ray absorption spectrum contains information about the local structure and chemistry of the excited atoms.

If the experimentally collected x-ray absorption spectroscopy can be succesfully simulated, through the modeling of the crystal structure, local structural and chemical quantities of the analyzed sample, such as oxidation states and coordination numbers, can be determined. This is the main procedure followed in this work, in order to answer open questions about the structural properties of various borides. To simulate the $\mathrm{x}-$ ray absorption spectrum for any given structural model, one requires full knowledge of its electronic structure. A method that has been particularly successful in calculating electronic structures for a whole range of materials and has seen an exponential growth in its use in the last two decades, is density functional theory. 
a) Incident photon
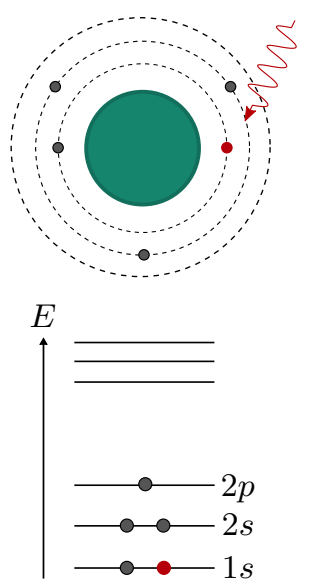

b) Excitation
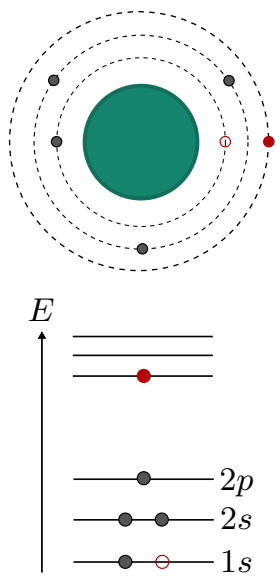

c) Auger decay
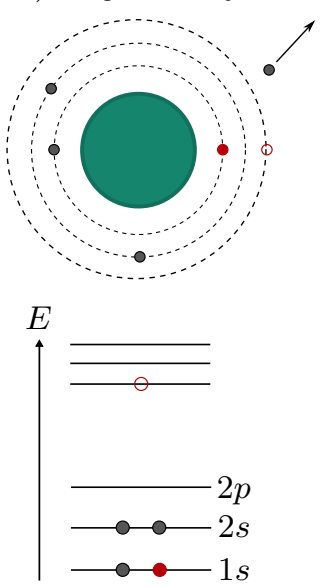

d) Fluorescent decay
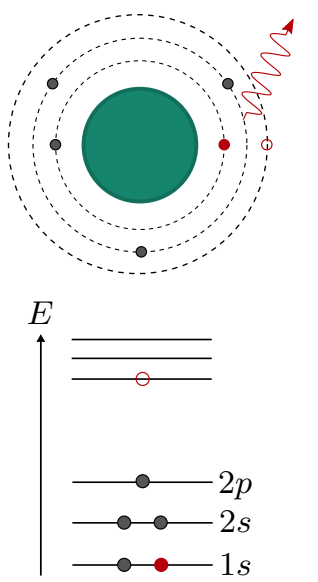

FiguRE 1.3: Going from left to right, there are four panels schematicaly representing the steps in the x-ray absorption spectroscopy process, specifically for the $K$-edge of a boron atom. For each panel, the top half represents the boron atom: the big circle indicating the nucleus, the dotted circles indicating the electronic orbitals and the small circles representing the electrons. The bottom half is a different representation of the electronic structure of the boron atom, where the electrons and their corresponding core levels are plotted along an energy scale. Each horizontal line represents a single electronic state that can be occupied by up to two electrons. (a) The initial condition shows a boron atom with its ground state electron configuration $1 s^{2} 2 s^{2} 2 p^{1}$ and an incident x-ray photon. (b) Given enough energy, the $\mathrm{x}$-ray photon is absorbed by one of the $1 s$ electrons and excited in an available empty state, leaving a hole behind in the core level. (c) The excited electron can decay into the core hole, transferring the accompanying release of energy to another electron which subsequently gets ejected. The intensity of ejected secondary electrons is the measured quantity in the total electron yield (TEY) mode of XANES. (d) Alternatively, the energy difference in the excited electron decay process can be released in the form of a fluorescence photon. The intensity of fluorescence photons is the measured quantity in the fluorescence yield (FLY) mode of XANES. 


\subsection{DENSITY FUNCTIONAL THEORY}

In order to describe the electronic structure of a given system, one has to venture into the domain of quantum mechanics. In quantum mechanics, any many-body system is described in full by its many-body wavefunction $\Psi$, the time-evolution of which is given by a famous linear partial differential equation, known as the Schrödinger equation[26$28]$

$$
i \hbar \frac{\partial}{\partial t} \Psi(\mathbf{r}, t)=\hat{H} \Psi(\mathbf{r}, t),
$$

where the Hamiltonian $\hat{H}$ is an operator that acts on the wave function and is determined by the many-body system itself. Determining the exact solution to this equation for a given system, would give access to all the properties of said system. In the world of theoretical physics then, quantum mechanical many-body problems form an interesting class of problems, those where the necessary equations to obtain an exact answer are known. However, the uncomplicated appearance of the Schrödinger equation is deceptive, and particle interactions in the many-body system make solving the equation analytically intractable for all but the smallest of systems.

During the late twenties of the twentieth century, in an attempt to make solving the Schrödinger equation for many-body systems tractable, Thomas and Fermi published a model where the properties of a system were described by its electronic density, replacing the many-body wave function as the central variable[29]. In their model, the total energy of the system was described as a functional of the electron density and hence came to be known as the density functional formalism. The model suffered from many deficiencies[30] and it was not until the work of Hohenberg and Kohn in the sixties, that the principle of the electronic density as a basic variable in many-body problems, was placed onto a sound theoretical foundation, which would come to be known as density functional theory (DFT).

In their seminal 1964 paper, Hohenberg and Kohn published two theorems that form the foundation of DFT[31]. The first theorem states, that for an inhomogeneous interacting electron gas in an external potential $V(\mathbf{r})$, the potential $V(\mathbf{r})$ is a unique functional of the ground state electronic density $n(\mathbf{r})$ of the electron gas, which was proven following a reductio ad absurdum argument. The assumption of the existence of a different external potential $V^{\prime}(\mathbf{r})$, having a different ground state $\Psi^{\prime}$, yet giving rise to the same electronic density $n(\mathbf{r})$, was shown to lead to inconsistencies. Combining the conclusion that $V(\mathbf{r})$ is a unique functional of $n(\mathbf{r})$ and the fact that $V(\mathbf{r})$ fixes the Hamiltonian $\hat{H}$ in Eq. 1.1, it follows that the full many-body wave function is a unique functional of the charge density $n(\mathbf{r})$.

The second Hohenberg and Kohn theorem states that an energy functional can be defined for the system, which is minimized by the correct ground state electron density. Obtaining the ground state electron density is still an intractable many-body problem 
due to the interacting nature of the electrons in the static external potential. A transformation, devised by Kohn and Sham[32], of the fully interacting electron system in the real potential, onto a system of non-interacting electrons in an effective potential, transforms the many-body Schrödinger equation for a system with $N$ particles, into $N$ independent single particle Kohn-Sham equations. The tractability of solving this fictitious system of decoupled equations is greatly improved and the real ground state density can be reconstructed from its solutions. This gives DFT a strong advantage over for example all-electron approaches that are simply too computationally intensive to be applied practically to system sizes required for real world examples.

The work of Hohenberg, Kohn and Sham is nowadays widely considered to form the foundation of modern DFT, however its future success was certainly not realized at the time of its conception[30]. It was not until the eighties, that Kohn-Sham density functional theory (KS-DFT) saw a sudden explosive growth in research activity, with an average of less than 100 publications per year just before the nineties[33], growing to almost 17000 publications in 2014[30]. The total amount of notable DFT publications is conservatively estimated to already exceed 150000 [34] and when ranking the most cited papers across all scientific disciplines, no less than twelve DFT publications rank in the top hundred[35]. With a comparable explosive growth in available computing power, made possible by continuous advances in the field of photolithography, producing ever faster computer microprocessors, model systems that can reasonably be treated within the DFT framework, have seen an increase in size, from just a handful of atoms to as many as a few thousand atoms. As further innovations in raw computational power and algorithm efficiency keep pushing the limiting boundaries forward, an ever increasing collection of real world systems can be studied successfully within the density functional theory framework.

\subsection{X-RAY ABSORPTION SPECTROSCOPY FROM FIRST PRINCIPLES}

\subsubsection{FERMI'S GOLDEN RULE}

As described in Section 1.2, the process that is studied in x-ray absorption spectroscopy, is the characteristic interaction between an x-ray photon of a given energy and a core electron of a certain element. Incoming photons have a probability of exciting core electrons into an empty state, creating electron-hole pairs, and subsequent measurements of the intensity of fluorescent photons or secondary electrons, that accompany the decay process of the excited states, are an indirect measure of that excitation probability. The transition rate of a core level electron from an initial state $|i\rangle$ into a final excited state $|f\rangle$ due to the absorption of a photon of energy $\hbar \nu$, is given by Fermi's golden rule:

$$
\Gamma_{i \rightarrow f} \propto \frac{2 \pi}{\hbar}|\langle f|\hat{H}| i\rangle|^{2} \delta\left(E_{f}-E_{i}-\hbar \nu\right)
$$


where $\hat{H}$ is the perturbing Hamiltonian that describes the interaction process and the delta function ensures that energy is conserved by requiring that the energy difference between the final and initial state $E_{f}-E_{i}$ is equal to the photon energy $\hbar \nu$. In the case of electron-photon interaction, the perturbing Hamiltonian takes the form of a dot product between the momentum operator and the vector potential of the electromagnetic field, allowing Eq. 1.2 to be rewritten to

$$
\Gamma_{i \rightarrow f} \propto \frac{2 \pi}{\hbar}\left|\left\langle f\left|\hat{\mathbf{p}} \cdot \vec{e} e^{-i \vec{k} \cdot \vec{r}}\right| i\right\rangle\right|^{2} \delta\left(E_{f}-E_{i}-\hbar \nu\right)
$$

where $\hat{\mathbf{p}}$ is the momentum operator, $\vec{e}$ is the polarization vector of the electric field and $\vec{k}$ is the photon momentum. In the dipole approximation, the exponential term can be expanded and in the zeroth order approximation, where $\vec{k} \cdot \vec{r} \gg 1$, the definition can be simplified to

$$
\Gamma_{i \rightarrow f} \propto \frac{2 \pi}{\hbar}|\langle f|\hat{\mathbf{r}} \cdot \vec{e}| i\rangle|^{2} \delta\left(E_{f}-E_{i}-\hbar \nu\right)
$$

The dipole approximation holds for regimes where the wavelength of the photon is much larger than the length scale of the atom. This condition holds for all the core level absorption processes considered in this work, where the incident photons typically have an energy of a few hundred $\mathrm{eV}$.

It is now clear, that in order to compute the excitation probability, one needs to evaluate the matrix elements $|\langle f|\hat{\mathbf{r}} \cdot \vec{e}| i\rangle|^{2}$, which requires knowledge of the initial and final states of the electron. Section 1.3 described how density functional theory can solve the electronic structure problem, which could therefore be used to provide the initial state and every potential final state that one could be interested in.

\subsubsection{THE PSEUDOPOTENTIAL PLANE WAVE IMPLEMENTATION}

The density functional theory formalism can in principle be implemented in a variety of ways, regarding the representation of for example the wave functions and the Coulomb potentials of the atomic nuclei. Typical choices for basis sets into which the wave functions are expanded, are Gaussian orbitals, local atomic orbitals and plane waves. Since the systems considered in this work are all solid state crystalline systems, the most logical choice for the basis set functions are plane waves.

A downside of the plane wave approach is that wave functions of core and valence electrons often have rapid oscillations near the nucleus. To describe these high frequency oscillations accurately a large basis set, i.e. a large number of plane waves is required, which significantly increases the computational time required of solving the electronic structure problem. One possible approach to alleviate this problem is the pseudopotential approach, which replaces the explicit description of the atomic nucleus and the core electrons with an effective potential, under the assumption that the character of core states is largely independent of the environment of the atom. The combination 
of the plane wave and pseudopotential approach is used in this work for all the x-ray absorption spectroscopy calculations.

With the pseudopotential plane wave framework, the electronic structure problem can be solved efficiently and the electronic density, from which the x-ray absorption spectroscopy is calculated, can be determined. However, the electronic density as found by DFT, corresponds by definition to the ground state density, whereas the final state in the absorption process with a core electron in an excited state, is clearly not a ground state of the system. Any changes that the system would undergo in the presence of the excited core electron, are therefore not captured by the electronic density as determined from DFT and the influence of the excited state is known to be significant in many cases, with borides forming no exception.

\subsubsection{EXCITED ELECTRON CORE-HOLE APPROACH}

The method used in this work, to model the Coulomb interaction of the excited electron with the created core-hole and the rest of the system, is called the excited electron corehole $(\mathrm{XCH})$ approach[36]. In the pseudopotential plane wave framework, the core hole is simulated directly by creating a pseudopotential in which the relevant core electron is removed. The core electron is then placed in the first available empty orbital and under constraints of those electron occupations, the electronic density is solved selfconsistently.

This simple approach has been shown to be very successful in capturing electron-hole interactions in the core level spectroscopy of first-row elements in a wide range of systems: from the description of x-ray absorption spectroscopy of water and ice[36], to the structure of the electronic double layer of water at a gold interface[37]. From the spectroscopy of aqueous boron oxides[38] and carbon dioxides[39], to the structure of polysulfide radicals in lithium batteries[40] and the structure of complex metalloorganic frameworks used in gas capture applications[41]. Due to the success of the DFT$\mathrm{XCH}$ approach in modeling the x-ray absorption near-edge spectroscopy for these light elements, it has been selected to describe electron-hole interactions in the theoretical modeling of x-ray spectroscopy of the solid state borides, described in this work.

\subsection{EXPERIMENTAL X-RAY ABSORPTION SPECTROSCOPY}

All experimental x-ray absorption spectroscopy measurements presented in this work, were carried out at beam line 6.3.2 of the Advanced Light Source at Lawrence Berkeley National Laboratory, which has been described in detail elsewhere[42, 43]. This beam line is usually used as a reflectometer but it also has the capabilities to measure the current generated through secondary electrons with a GaAsP photodiode. Due to the low atomic numbers of the elements studied, all experiments were conducted in 
the total electron yield mode, as the fluorescence decay channel is relatively weak for low- $Z$ elements.

The beam line has a wavelength range from $25 \mathrm{eV}$ to $1300 \mathrm{eV}$, which includes the energies of the boron $K$-edge and the phosphorus $L_{2,3}$-edge at $188 \mathrm{eV}$ and $130 \mathrm{eV}$, respectively, studied in this work. The synchrotron radiation is passed through an array of optical elements, including a grating to select the required wavelength and an order suppressor to reduce intensity from higher orders, resulting in a monochromatic and linearly polarized beam with a spectral purity of approximately $99.98 \%$. Before each measurement the photon energy of the beam was calibrated with respect to the absorption edges of calibrated silicon and boron filters, installed at the beam line. With a wavelength precision of approximately $0.01 \%$, the beam line gives an energy resolution of approximately $0.02 \mathrm{eV}$, which is more than sufficient for the application required in this work.

After each measurement, the dark current, intrinsic to the photodiode in the absence of incident photons, and the direct beam spectrum, to account for fluctuations in intensity of the photon beam as a function of photon energy, are measured. The dark current is subtracted from the collected spectrum and is subsequently normalized with respect to the direct beam. The final intensity resolution is approximately $0.1 \%$, which allows the setup to detect defect populations down to the order of approximately $0.5 \%$. For the work presented in this thesis, this resolution is more than sufficient as will be discussed in more details in the following chapters.

\subsection{OUTLINE}

In Chapter 2, the computational method employed in this work, which focuses on the DFT-XCH method, will be described in detail. The described method will be applied in the following chapters to describe the structure and chemistry of various borides.

In Chapter 3, the DFT-XCH method will be applied to study the boron $K$-edge of hexagonal boron nitride, a material whose x-ray spectroscopy has been studied extensively in literature, and therefore serves as a perfect benchmark to determine the efficacy of DFT-XCH in predicting boron $K$-edge absorption spectroscopy. In addition to calibrating the DFT-XCH method, we will present novel details about the properties and origins of structural defects often found in $h$-BN, through the study of the measured and simulated absorption spectroscopy. The results will show that oxygen plays an integral role in the formation of triangular voids in the hexagonal boron nitride crystal structure.

With the proposed computational method successfully benchmarked, we move on to a different boride, namely $\mathrm{B}_{12} \mathrm{P}_{2}$, to try and unveil the origins of the mysterious "selfhealing" property found in this material. Before the x-ray absorption spectroscopy of 
$\mathrm{B}_{12} \mathrm{P}_{2}$ is discussed, firstly the properties of crystalline point defects are described in Chapter 4. This is a purely theoretical investigation that employs nudged elastic band (NEB) calculations to investigate which defects are likely to form in $\mathrm{B}_{12} \mathrm{P}_{2}$ and subsequently, how created antisite and vacancy defects may recombine to restore structural damage. The results will show that due to the icoshedral boron units in $\mathrm{B}_{12} \mathrm{P}_{2}$, the crystal structure remains stable even in the presence of point defects and that the activation energy for defect recombination can be as low as $3 \mathrm{meV}$. The suprisingly shallow barriers explain how any created defects can appear to be restoring themselves automatically, explaining the experimentally observed "self-healing" in icosahedral borides.

In Chapter 5, the x-ray absorption spectroscopy of icosahedral boron phosphide samples will be presented. Theoretical modeling of spectroscopic signatures of various possible point defects in the $\mathrm{B}_{12} \mathrm{P}_{2}$ crystal structure, reveals that the studied samples have a low amount of boron vacancies, which is perfectly congruent with the defect recombination mechanism discovered and described in Chapter 4. The predicted defect spectroscopic signatures serve as defect fingerprints to be used in future studies that would monitor defect creation and recombination in situ.

Chapter 6 describes the work performed on the cubic phase of boron phosphide $c$-BP. The experimentally collected x-ray absorption spectroscopy for samples synthesized with chemical vapor deposition is modeled with the DFT-XCH method. A perfect $c$-BP crystal is found to accurately model the $\mathrm{P} L_{2,3}$-edge, however fails to reproduce the unusually broad and gradual absorption onset of the experimental B $K$-edge spectrum. Various model refinements are discussed, from which it follows that the most likely candidate for the broad absorption onset is the presence of amorphous boron clusters within the cubic boron phosphide network. These new insights provide valuable information to improve synthesis methods that aim to produce high quality boron phosphide crystals.

The final chapter is a valorization chapter, in which the design and development of a graphical user interface called WEBXS is described. WEBXS was built to give users of the Molecular Foundry user facility easy access to the academic software developed and used in this thesis work to simulate x-ray absorption spectroscopy from first principles. Academic codes are often characterized by an enormous potential of generating valuable novel insights at the cost of a steep learning curve and the requirement of a variety of skills to operate them. WEBXS makes this software available to non-experts with relative ease, giving access to powerful theoretical analysis of experimentally collected x-ray absorption spectroscopy data with relatively little to no formal training. 


\section{REFERENCES}

[6] T. Ogitsu, F. Gygi, J. Reed, Y. Motome, E. Schwegler, and G. Galli, J. Am. Chem. Soc. 131, 1903 (2009).

[7] K. S. Novoselov, Science 306, 666 (2004).

[8] C. Zhi, Y. Bando, C. Tang, H. Kuwahara, and D. Golberg, Adv. Mater. 21, 2889 (2009).

[9] L. Song, L. Ci, H. Lu, P. B. Sorokin, C. Jin, J. Ni, A. G. Kvashnin, D. G. Kvashnin, J. Lou, B. I. Yakobson, and P. M. Ajayan, Nano Letters 10, 3209 (2010).

[10] Y. Chen, J. Zou, S. J. Campbell, and G. Le Caer, Applied Physics Letters 84, 2430 (2004).

[11] A. Zunger, A. Katzir, and A. Halperin, Phys. Rev. B 13, 5560 (1976).

[12] K. K. Kim, A. Hsu, X. Jia, S. M. Kim, Y. Shi, M. Hofmann, D. Nezich, J. F. Rodriguez-Nieva, M. Dresselhaus, T. Palacios, and J. Kong, Nano Letters 12, 161 (2012).

[13] C. R. Dean, A. F. Young, I. Meric, C. Lee, L. Wang, S. Sorgenfrei, K. Watanabe, T. Taniguchi, P. Kim, K. L. Shepard, and J. Hone, Nature Nanotech 5, 722 (2010).

[14] J. C. Meyer, A. Chuvilin, G. Algara-Siller, J. Biskupek, and U. Kaiser, Nano Letters 9, 2683 (2009).

[15] M. Carrard, D. Emin, and L. Zuppiroli, Phys. Rev. B 51, 11270 (1995).

[16] D. Emin, Journal of Solid State Chemistry 179, 2791 (2006).

[17] J. C. Lund, F. Olschner, F. Ahmed, and K. S. Shah, MRS Proc. 162 (1989) $10.1557 /$ proc-162-601. 
[18] T. P. Viles, B. A. Brunett, H. Yoon, J. C. Lund, H. Hermon, D. Buchenauer, K. McCarty, M. Clifft, D. Dibble, and R. B. James, MRS Proc. 487 (1997) 10.1557/proc-487-585.

[19] Y. Y. Platonov, L. Gomez, and D. Broadway, in X-ray mirrors, crystals, and multilayers II, edited by A. K. Freund, A. T. Macrander, T. Ishikawa, and J. L. Wood, (Dec. 2002).

[20] T. Tsarfati, R. van de Kruijs, E. Zoethout, E. Louis, and F. Bijkerk, Thin Solid Films 518, 1365 (2009).

[21] I. A. Makhotkin, E. Zoethout, R. van de Kruijs, S. N. Yakunin, E. Louis, A. M. Yakunin, V. Banine, S. Müllender, and F. Bijkerk, Opt. Express 21, 29894 (2013).

[22] V. Medvedev, A. Yakshin, R. van de Kruijs, and F. Bijkerk, Optical Materials Express 5, 1450 (2015).

[23] B. Padavala, C. D. Frye, X. Wang, Z. Ding, R. Chen, M. Dudley, B. Raghothamachar, P. Lu, B. N. Flanders, and J. H. Edgar, Crystal Growth \& Design 16, 981 (2016).

[24] B. Padavala, C. Frye, X. Wang, B. Raghothamachar, and J. Edgar, Journal of Crystal Growth (2016) 10.1016/j.jcrysgro.2016.05.031.

[25] J. A. Bearden, and A. F. Burr, Reviews of Modern Physics 39, 125 (1967).

[26] E. Schrödinger, Phys. Rev. 28, 1049 (1926).

[27] E. Schrödinger, Ann. Phys. 384, 489 (1926).

[28] E. Schrödinger, Ann. Phys. 385, 437 (1926).

[29] L. H. Thomas, Math. Proc. Camb. Phil. Soc. 23, 542 (1927).

[30] R. O. Jones, Reviews of Modern Physics 87, 897 (2015).

[31] P. Hohenberg, and W. Kohn, Phys. Rev. 136, B864 (1964).

[32] W. Kohn, and L. J. Sham, Phys. Rev. 140, A1133 (1965).

[33] K. Burke, The Journal of Chemical Physics 136, 150901 (2012).

[34] A. D. Becke, The Journal of Chemical Physics 140, 18A301 (2014).

[35] R. V. Noorden, B. Maher, and R. Nuzzo, Nature 514, 550 (2014).

[36] D. Prendergast, and G. Galli, Phys. Rev. Lett. 96, 215502 (2006).

[37] J.-J. Velasco-Velez, C. H. Wu, T. A. Pascal, L. F. Wan, J. Guo, D. Prendergast, and M. Salmeron, Science 346, 831 (2014).

[38] A. M. Duffin, C. P. Schwartz, A. H. England, J. S. Uejio, D. Prendergast, and R. J. Saykally, The Journal of Chemical Physics 134, 154503 (2011).

[39] A. H. England, A. M. Duffin, C. P. Schwartz, J. S. Uejio, D. Prendergast, and R. J. Saykally, Chemical Physics Letters 514, 187 (2011). 
[40] T. A. Pascal, C. D. Pemmaraju, and D. Prendergast, Phys. Chem. Chem. Phys. 17, 7743 (2015).

[41] W. S. Drisdell, R. Poloni, T. M. McDonald, J. R. Long, B. Smit, J. B. Neaton, D. Prendergast, and J. B. Kortright, J. Am. Chem. Soc. 135, 18183 (2013).

[42] J. Underwood, and E. Gullikson, Journal of Electron Spectroscopy and Related Phenomena 92, 265 (1998).

[43] E. M. Gullikson, S. Mrowka, and B. B. Kaufmann, Recent developments in euv reflectometry at the advanced light source, 2001. 


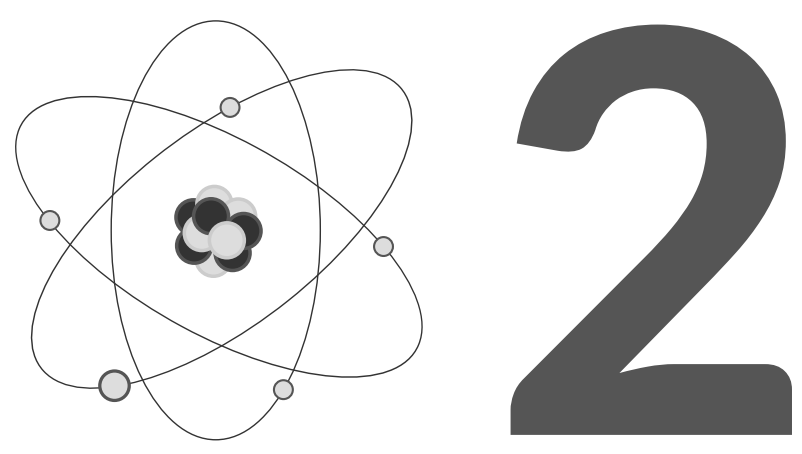

COMPUTATIONAL METHOD 


\section{ABSTRACT}

A global description of the complete computational method to compute the x-ray absorption spectrum for a given material, as used in this work, will be presented in this chapter. As an example, the boron $K$-edge for hexagonal boron nitride $(h$-BN) will be calculated, which will recurr and be analyzed in greater detail in Chapter 3. 


\subsection{CRYSTAL STRUCTURE DEFINITION}

The first requirement for any DFT-XCH calculation, is a description of the atomic or crystallographic structure of the material or system for which one wants to compute the x-ray absorption spectrum (XAS). In the case of $h$-BN this means a definition of its crystal structure, which is defined by a hexagonal unit cell that contains 2 boron and 2 nitrogen atoms. First, the unit cell parameters and atomic positions need to be optimized, by minimizing the total energy of the system with respect to these parameters. All structural optimizations and atomic relaxations are performed within the DFT framework with the Vienna ab initio simulation package VASP[44-47].

The DFT-XCH method employs periodic boundary conditions to simulate a solid that stretches out into infinity in all directions, from a simple finite structure definition. However, this leads to artificial interactions between structural anomalies and their own mirror images reflected in the periodic boundary conditions. An example of such an anomaly is a core excited atom, as in the case of x-ray absorption simulations. To minimize these artifical self-interactions, the dimensions of the unit cell that defines the structure, need to be sufficiently large.

In our example of $h$-BN, the unit cell of 4 atoms is definitely too small and would be prone to self-interaction errors and therefore a supercell needs to be created. A schematic representation of a fully optimized and relaxed $h$-BN supercell structure is shown in Fig. 2.1 (a). A crystal structure is optimized, by minimizing the total energy of the structure as a function of the crystal lattice parameters. After the crystal cell is optimized, the structure is relaxed by minimizing the forces that act on the individual atoms as a function of their position within the crystal lattice. The dimensions of the supercell in Fig. 2.1 are sufficiently large, such that any fictitious effects introduced by the periodic boundary conditions are minimal and the computed x-ray absorption spectrum is converged.

To simulate the B $K$-edge absorption spectrum for this crystal structure, one first needs to create an excited configuration by replacing a single boron atom with a core excited atom that has a hole in the $1 s$ core level. The choice of which boron atom to excite is arbitrary in this particular case, since the structure is represented by the perfect crystal structure and the symmetry of the hexagonal crystal lattice dictates that all boron atoms are symmetrically equivalent. Therefore one only has to compute the absorption spectrum for a single excited configuration as all possible configurations would be symmetrically equivalent and yield identical absorption spectra. The computed XAS represents the spectrum for the static structure and therefore effectively simulates the structure's spectroscopy at an effective temperature of absolute zero. However, experimental x-ray absorption spectroscopy measurements are typically not conducted at absolute zero and they sample a statistical average over time of a large collection of illuminated atoms, while they are displaced from their equilibrium crystal 
a) $\quad h$-BN static $T=0 \mathrm{~K}$

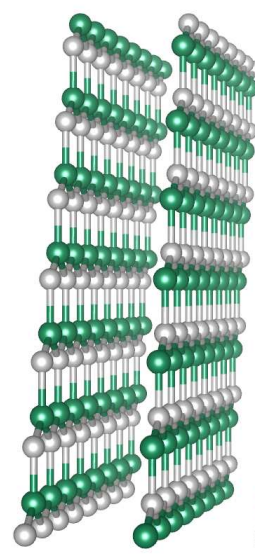

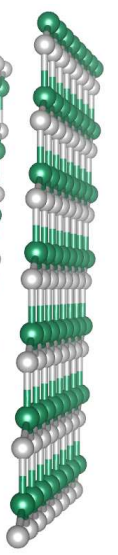

Boron

Nitrogen b) $\quad h$-BN thermal $T=300 \mathrm{~K}$

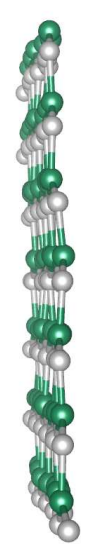

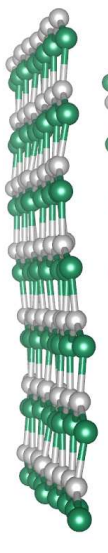

Figure 2.1: (a) A representation of a fully optimized and relaxed $h$-BN supercell structure at a temperature of $0 \mathrm{~K}$. Boron and nitrogen atoms are represented by green and light gray colored spheres, respectively. Boron nitrogen bonds are drawn up to a distance of $1.6 \AA$. (b) A single snapshot taken from a molecular dynamics trajectory of the same boron nitride supercell, equilibrated at a temperature of $300 \mathrm{~K}$. Notice how the overall crystal structure is maintained, but all atoms are locally slightly displaced from their optimal crystal lattice position.

positions by thermally induced lattice vibrations. The DFT-XCH method can not directly capture these dynamic processes as it solves the time-independent Schrödinger equation, however the thermal effects on the structure can be modeled separately, for example by means of molecular dynamics.

\subsection{AB-INITIO MOLECULAR DYNAMICS}

The divergence of atomic positions from their equilibrium crystal lattice positions due to thermally induced lattice vibrations, can be simulated with ab-initio molecular dynamics. For a given structure, the forces acting on each atom can be derived quantum mechanically from the electronic density as obtained from DFT, which can subsequently be used to solve Newton's equation of motion classically. Using a simple Verlet algorithm, the atomic equations of motion can be integrated for a certain length of time, called the timestep, in order to find the new atomic positions. It is important to note that during the movement of the atoms, the forces acting on them are not being updated, but rather remain constant and therefore too long a timestep will lead to errors in the atomic motions. For light elements like boron atoms, typical appropriate timestep values are of the order of 0.1 fs to $1 \mathrm{fs}$. After extensive testing, by monitoring the drift in total energy over time as a function of the used time step, a time step of 
$0.2 \mathrm{fs}$ was used throughout the entirety of this work, which guarantees an energy drift below $0.05 \mathrm{meV} \mathrm{ps}^{-1}$ per atom.

The temperature during the molecular dynamics can be regulated by coupling the system to a thermostat. In this work, we employ a Noseé-Hoover thermostat, which will control the temperature of the system by adding or extracting kinetic energy to the system, by exchanging atoms with a reservoir. By removing or adding atoms with a velocity above the effective temperature, the temperature of the system can be lowered or increased, respectively. The specific temperature that is required, depends on the experimental conditions that are to be simulated.

Like all structural optimizations, all molecular dynamics simulations in this work, are carried out using VASP. Figure 2.1 (b) shows a graphic representation of the structure of a $h$-BN supercell, taken at a random point in time, during a molecular dynamics simulation,where the system was equilibrated at a temperature of $300 \mathrm{~K}$. Such a structural configuration, taken from a molecular dynamics trajectory, representing the dynamic supercell structure frozen in time, is commonly referred to as a "snapshot". Notice how, compared to the perfect crystal structure depicted in Fig. 2.1 (a), the overall crystal structure is maintained, but every atom is slightly displaced from its original equilibrium position. Consequently, in stark contrast with the perfect crystal structure described in the previous section, the boron atoms are no longer perfectly symmetrically equivalent. To compute the total x-ray absorption spectrum for this snapshot, each boron atom should be replaced separately by a core-excited atom, creating multiple excited configurations for which the x-ray absorption spectrum is calculated. Averaging the resulting individual atomic spectra will yield the total spectrum of the snapshot.

In repeating this process for multiple snapshots, each taken from different points in time during the molecular dynamics simulation, an average spectrum for multiple snapshots, each having a slightly different atomic configuration, can be computed. This method of modeling the distortion of atomic positions due to thermal vibrations and the indirect effect it has on their individual atomic absorption spectrum over multiple snapshots, simulates the statistical averaging over multiple excited atoms and time, that naturally takes place in experimental spectroscopy measurements. As a result, the simulated "thermal" spectroscopy often describes experiment better than the simple "static" spectroscopy simulated for the perfect crystal structure in the absence of thermal effects. However, this comes at an expense as the computational cost is increased significantly by a factor equal to the number of snapshots, multiplied by the number of atoms in each snapshot.

\subsection{CALCULATING THE ELECTRONIC STRUCTURE}

With a structural configuration of the system of interest in hand, be it a relaxed crystal structure or a snapshot from a molecular dynamics trajectory, its electronic 
structure can now be computed within the framework of density functional theory. We employ the plane wave and pseudopotential based DFT implementation QUANTUM EsPrEsso[48]. The pseudopotential of the atom that is to be excited is replaced by a pseudopotential with the relevant core electron explicitly removed; sticking to our example of the simulation of the boron $K$-edge, one of the $1 s$ electrons is removed. The removed electron is placed in the lowest energy unoccupied orbital in order to simulate the excited electron. Under the constraint of fixed electronic occupancies of this initial condition, the electronic density is now solved self-consistently with a standard selfconsistent field calculation. This calculation can be performed with just a small number of empty states to speed up the convergence of the calculation. After convergence is reached, the computed charge density can be used for a non self-consistent field calculation with an increased number of empty states, which will give the electronic structure of the system.

\subsection{OPTIMIZED BASIS SETS}

From the solved electronic structure, it is in principle now possible to compute the transition probability of an electron from state $|i\rangle$ to $|f\rangle$ by evaluating Fermi's golden rule in the dipole approximation, as defined in Eq.1.4. To that end, the transition probability matrix elements that enter in that equation have to be computed, which requires the diagonalization of the matrix

$$
\hat{H} \equiv\langle f|\hat{\mathbf{r}} \cdot \vec{e}| i\rangle
$$

For a large system with many empty electronic states and a dense plane wave basis set, diagonalizing this matrix can be computationally prohibitive. To reduce the computational cost significantly, we employ the method devised by Shirley, which determines a smaller subset of periodic basis functions, that still adequately spans the space spanned by the original plane wave basis set[49]. By solving Schrödinger's equation at a select few high symmetry $k$-points in the first Brillouin zone, one obtains an optimal basis set for all electron states that can be used for integration anywhere in the Brillouin zone. After obtaining the optimal basis set, the Hamiltonian $\hat{H}$ is projected onto this new basis set and diagonalized to obtain the transition probability matrix elements. From the computed matrix elements, Eq. 1.4 can be used to compute the transition probability for a set of desirable final states $|f\rangle$.

\subsection{POST-PROCESSING OF COMPUTED ABSORPTION SPECTRUM}

An example of a computed absorption spectrum is given for the perfect crystal structure of $h$-BN, shown in Fig. 3.3(a). The computed spectrum is defined by a set of delta functions labeled "raw spectrum", representing the transition probability of the 
core electron into the final state as a function of the final states relative energy. To arrive at the final spectrum shown in red, the delta functions of the raw spectrum are convoluted with Gaussian funtions. The resulting broadening can be controlled by selecting the width of the Gaussian functions used in the convolution. To compensate for the infamous intrinsic underestimation of the band gap by the generalized-gradient approximation in density functional theory, the energy scale can be dilated by a certain factor to align with experimental results if necessary.

The eigenvalues of electronic states obtained within DFT are purely relative energy values, due to the absence of an absolute energy reference point, like in all-electron calculations. This also implies that the relative energy scales of spectra obtained for different excited configurations from the same structural snapshot, are not necessarily aligned and can not be compared directly. To provide a common reference point, we adopt an energy alignment scheme, which references all computed eigenvalues with respect to the relative differences between the total energies of the system and the isolated excited atom in both its ground and excited state[39]. For a given structural configuration $\Sigma$ with an excited atom of type $X$, the energy $E$ of a particular excited state $f$ is derived from its Kohn-Sham eigenvalue $\epsilon_{f}$ through the following relation

$$
E=\epsilon_{f}-\epsilon_{0}+\left(E_{\Sigma}^{\mathrm{XCH}}-E_{X}^{\mathrm{XCH}}\right)-\left(E_{\Sigma}^{\mathrm{GS}}-E_{X}^{\mathrm{GS}}\right),
$$

where $\epsilon_{0}$ is the Kohn-Sham eigenvalue of the first unoccupied state that will be occupied by the excited electron, $E_{\Sigma}^{\mathrm{XCH}}$ and $E_{X}^{\mathrm{XCH}}$ are the total energies of the total system $\Sigma$ and an isolated atom of type $X$, respectively, when in the excited state. The same defintion applies to $E_{\Sigma}^{\mathrm{GS}}$ and $E_{X}^{\mathrm{GS}}$, but for the system and the atom in their ground state. This approach works well in general, but can fail in certain situations. This approach does not correct for self-interaction errors, however if the error is similar for all systems, the error will cancel out. For many systems this is the case, however for systems containing defects for example, the self-interaction error can differ drastically which may cause the alignment scheme to fail. This problem is encountered and addressed in detail in Chapter 5.

The spectrum shown in Fig. 3.3 (a) is an example of a computed spectrum that has been aligned according to previously described scheme and consequently it can now be compared meaningfully with computed spectra for different structural configurations that also have been aligned using the same procedure. However, the energy alignment is still relative and lacks an absolute reference point, as can be seen from the fact that the spectrum which is supposed to represent the boron $K$-edge at approximately $192 \mathrm{eV}$, has its absorption edge at around $0 \mathrm{eV}$. The computed spectrum needs one final spectral aligment, by shifting it by a value $\Delta E$, in order to align it to an experimentally obtained absorption spectrum. The computed spectrum of Fig. 3.3 (a) was shifted by a value of $191.5 \mathrm{eV}$ to align it with the experimental B $K$-edge spectrum as shown in Fig. 3.3 (b). Is important to note, that this experimental alignment value $\Delta E$ is kept constant for all computed spectra of structural configurations of the same type. As an 

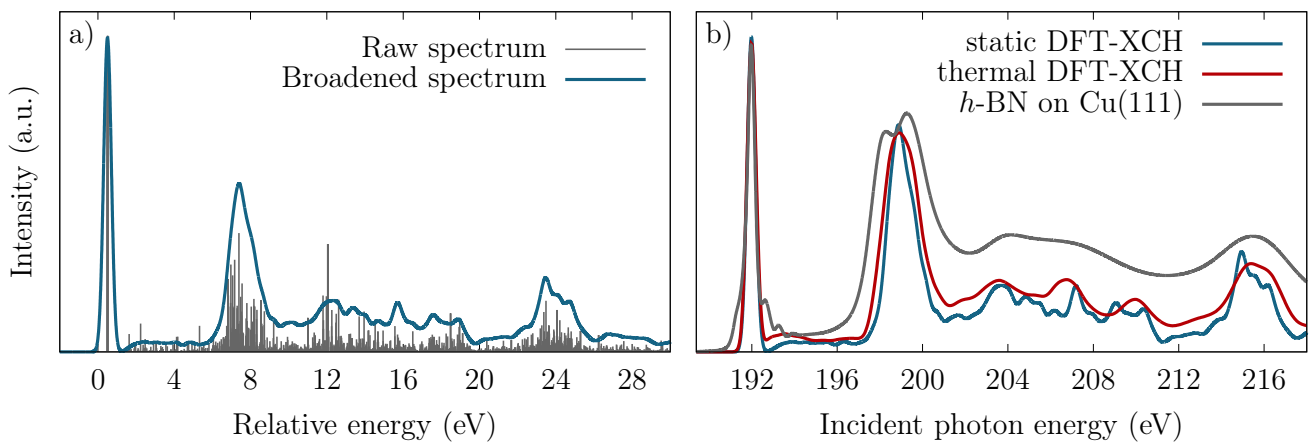

Figure 2.2: (a) The raw "stick" spectrum, indicated by the vertical grey lines, and the Gaussian convoluted broadened spectrum, represented by the solid red line, as calculated for the perfect crystal lattice of $h$-BN at a temperature of $0 \mathrm{~K}$. (b) The solid red line is identical to the broadened spectrum from panel (a) and the solid green line represents the averaged spectrum of multiple snapshots taken from a molecular dynamics trajectory of the same $h$-BN structure equilibrated at a temperature of $300 \mathrm{~K}$. The dashed grey line is an experimental B $K$-edge x-ray absorption spectrum collected for a $h$-BN sample, details of which are discussed in Chapter 3.

example, the solid grey line in Fig. 3.3 (b) labeled "thermal DFT-XCH", is an average spectrum computed from 5 different snapshots of a trajectory of a $h$-BN supercell equilibrated at a temperature of $300 \mathrm{~K}$. All the individual atomic spectra for each excited configuration of each snapshot, have been aligned according to Eq. 2.2 and subsequently shifted by $191.5 \mathrm{eV}$. As described in the last paragraph of Section 2.2 , the agreement of the thermal spectrum with experiment is improved compared to the static spectrum. Due to the indirect inclusion of thermal effects, the spectrum is broadened and smoothed, replicating the natural broadening found in experiment due to lattice vibrations.

There are still noticeable differences between the experimental and theoretical spectrum, but this should not come as a surprise as the thermalized perfect crystal structure is still an overly simplified model for what would be the real structure. Further improvements to the structural model such as the inclusion of crystalline point defects, grain boundaries or even amorphous phases will further improve the agreement between the measured and simulated spectrum. A potential source of inaccuracy in the simulated spectrum that can not be attributed to an incomplete structural model, is the employed exchange-correlation functional, which is an approximation inherent in the Kohn-Sham density functional theory framework. These approximation errors have to be evaluated for each specific case, but for the boride structures studied in this work, these errors do not play a significant role due to the lack of strongly correlated electrons. 


\section{REFERENCES}

[39] A. H. England, A. M. Duffin, C. P. Schwartz, J. S. Uejio, D. Prendergast, and R. J. Saykally, Chemical Physics Letters 514, 187 (2011).

[44] G. Kresse, and J. Hafner, Phys. Rev. B 47, 558 (1993).

[45] G. Kresse, and J. Hafner, Phys. Rev. B 49, 14251 (1994).

[46] G. Kresse, and J. Furthmüller, Computational Materials Science 6, 15 (1996).

[47] G. Kresse, and J. Furthmüller, Physical Review B 54, 11169 (1996).

[48] P. Giannozzi, S. Baroni, N. Bonini, M. Calandra, R. Car, C. Cavazzoni, D. Ceresoli, G. L. Chiarotti, M. Cococcioni, I. Dabo, A. D. Corso, S. de Gironcoli, S. Fabris, G. Fratesi, R. Gebauer, U. Gerstmann,

C. Gougoussis, A. Kokalj, M. Lazzeri, L. Martin-Samos, N. Marzari, F. Mauri, R. Mazzarello, S. Paolini, A. Pasquarello, L. Paulatto, C. Sbraccia, S. Scandolo, G. Sclauzero, A. P. Seitsonen, A. Smogunov, P. Umari, and R. M. Wentzcovitch, Journal of Physics: Condensed Matter 21, 395502 (2009).

[49] E. L. Shirley, Phys. Rev. B 54, 16464 (1996). 


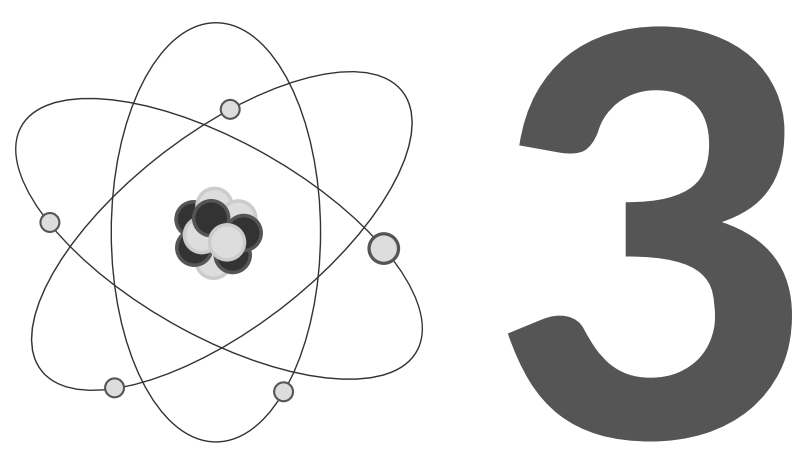

OXYGEN-STABILIZED TRIANGULAR DEFECTS IN HEXAGONAL BORON NITRIDE 


\section{ABSTRACT}

Recently several experimental transmission electron microscopy (TEM) studies have reported the observation of nanoscale triangular defects in mono- and multilayer hexagonal boron nitride $(h$-BN). First-principles calculations are employed to study the thermodynamical stability and spectroscopic properties of these triangular defects and the chemical nature of their edge termination. Oxygen-terminated defects are found to be significantly more stable than defects with nitrogen-terminated edges. Simulated x-ray absorption spectra of the boron $K$-edge for oxygen-terminated defects show excellent agreement with experimental x-ray absorption near-edge spectroscopy (XANES) measurements on defective $h$-BN films with oxygen impurities. Finally we show that the structural model for oxygen defects in $h$-BN as deduced from the simulated corelevel spectroscopy is intrinsically linked to the equilateral triangle shape of defects as observed in many recent electron microscopy measurements. 


\subsection{INTRODUCTION}

Hexagonal boron nitride $\left(h\right.$-BN) is an $s p^{2}$-bonded planar material and an isoelectronic structural analog of graphite with very similar lattice parameters. Like its carbon-based counterpart, $h$-BN has many interesting properties, such as high in-plane mechanical strength and thermal conductivity[8,9], and has been shown to have an even higher chemical stability compared to graphite[10]. Despite the many structural similarities between $h$-BN and graphite, however, there are also significant differences in material properties. Unlike the semimetal graphite, $h$-BN is a wide gap insulator[11], which allows it to be used as an ultraviolet emitter in optoelectronics[50]. Recently monolayer boron nitride $g$-BN has been successfully synthesized[12] and makes a great candidate for use in conjunction with graphene in novel electronics due to their structural commensurability but contrasting electronic properties[13].

A high degree of structural quality and integrity is crucial for these applications[51], however, recently several studies on structural defects in $h$-BN have been published[14, 52-54] that observe the formation of voids of various sizes with a very distinctive equilateral triangular shape. The methods employed to study these triangular structural imperfections and their formation are electron-microscopy based techniques such as annular dark field (ADF) imaging and transmission electron microscopy (TEM). The high spatial resolution of these techniques allows for a very accurate analysis of the structural and geometrical properties of the observed defects, but the marginal chemical sensitivity limits the capabilities to study chemical properties, specifically of the atoms located at the edge of the void created by the defects. In an effort to gain more insight into the chemical nature of the defects and their edge termination, recent studies have conducted scanning transmission electron microscopy electron energy loss spectroscopy (STEM-EELS) measurements to elucidate the spectroscopic signature of triangular voids at the nitrogen[54] et al. and boron $K$-edge[55]. The results show very characteristic features in the respective core-level spectra and indicate that spectroscopic methods are ideal candidates to investigate the true chemical nature of triangular defects in $h$-BN, which is still open to debate.

The early TEM studies report almost exclusively boron centric vacancies with nitrogenterminated edges, as confirmed by the STEM-EELS work of Suenaga[54], with the origin of this asymmetry being attributed to the lower knock-on threshold value of boron compared to that of nitrogen under the influence of the electron beam of the TEM measurement itself[56], while nitrogen centric vacancies have since also been shown to exist under certain experimental conditions[55]. In stark contrast, x-ray absorption near edge spectroscopy (XANES) studies on defective $h$-BN thin films have exclusively observed nitrogen voids[57] or oxygen impurity defects[58-60], as indicated by very prominent and distinguishing features in the boron $K$-edge spectrum. In this work we provide a solution for this apparent contradiction in literature by means of theoretical calculations from first-principles on the thermodynamic stability, chemical 
nature, and spectroscopic properties of triangular defects that apply to both bulk and monolayer $h$-BN. Our results reveal a direct link between the geometric properties of the experimentally observed vacancy-based defects and distinctive spectral features observed in core-level spectroscopy measurements.

\subsection{EXPERIMENTAL DETAILS}

All x-ray absorption spectroscopy measurements were carried out at beamline 6.3.2 of the Advanced Light Source (ALS) synchrotron at Lawrence Berkeley National Laboratory (LBNL). A detailed description and characterization of the beamline and measurement chamber can be found elsewhere[42, 43]. X-ray absorption measurements of the boron $K$-edge were collected in total electron yield (TEY) mode, from commercially available samples (Graphene Supermarket) of chemical vapor deposited (CVD) grown thin-films of $h$-BN on $\mathrm{Cu}(111)$ foil[51]. The $p$-polarized incident soft x-ray beam had an angle of incidence of $1.5^{\circ}$ with respect to the sample surface normal. Energy calibration was performed by comparing to absolute absorption edges of Si and B filters installed at the beamline. The collected spectra have the dark current signal subtracted to account for the systematic error and noise in the collector electronics. Subsequently, the spectra are normalized by a spectrum collected by a photodiode to account for the intensity fluctuations in the x-ray beam as a function of photon energy. Since TEY is a surface-sensitive technique and the $h$-BN films were $13 \mathrm{~nm}$ thick, the resulting spectra did not have to be corrected for collected electrons originating from the copper substrate.

\subsection{COMPUTATIONAL METHOD}

\subsubsection{STRUCTURAL RELAXATION AND MOLECULAR DYNAMICS}

All structural optimizations and molecular dynamics have been carried out within the DFT framework using the Vienna ab initio simulation package vASP[47]. Core electrons are replaced by ultrasoft pseudopotentials within the projector augmented wave (PAW) method[61, 62], and the $2 p$ and $2 s$ electrons for boron, nitrogen, and oxygen are treated as valence electrons. The generalized gradient approximation (GGA) as formulated by Perdew-Burke-Ernzerhof (PBE) [63] is employed for the exchange-correlation energy. A kinetic cutoff energy of $400 \mathrm{eV}$ was used for the plane waves. For the non-defective bulk calculations a $4 \times 4 \times 2$ supercell with a total of 128 atoms was constructed and the Brillouin zone was sampled at the $\Gamma$ point. For the defect calculations the supercell was increased to a $11 \times 11 \times 1$ for the bulk calculations to ensure the isolation of the defect from its periodic image. A $6 \times 6 \times 2$ cell with $10 \AA$ of vacuum along the $c$-axis of the cell was used for the surface cell calculations. van der Waals interactions were accounted for 
by applying the corrective scheme of Tkatchenko and Scheffler[64] as implemented in VASP. The supercell structures were optimized by minimizing the Hellmann-Feynman forces acting on the nuclei below the threshold value $0.02 \mathrm{eV}^{-1}$. Thermally induced structural distortions of the bulk $h$-BN crystal lattice were simulated by sampling the canonical ensemble (NVT) at a finite temperature of $300 \mathrm{~K}$ regulated by a Nosé-Hoover thermostat. The time step of integration was set to $0.2 \mathrm{fs}$. The system was thermally equilibrated for $50 \mathrm{ps}$, after which the micro canonical ensemble $(N V E)$ was sampled for an additional $10 \mathrm{ps}$, maintaining a temperature of $320 \mathrm{~K}$. From the last $10 \mathrm{ps}$ of the simulated trajectory, five snapshots, each separated by $2 \mathrm{ps}$, were taken to represent a statistical average of the structure at finite temperature.

\subsubsection{FORMATION ENERGIES}

The formation energy $E^{f}$ of a defect $X$ in charge state $q$ is commonly estimated by the equation[65]

$$
E^{f}\left[X^{q}\right]=E\left[X^{q}\right]+E_{\text {corr }}^{q}-E[\text { host }]-\sum n_{i} \mu_{i}+q\left(\epsilon_{F}+\epsilon_{v}+\Delta v\right),
$$

where $E\left[X^{q}\right]$ and $E[$ host $]$ are the total energies of the defective and pristine host structures, respectively, as derived from supercell calculations. The amount of particles of type $i$, both native and impure, that are added or removed is given by $n_{i}$, which is positive for a net amount of particles added and negative otherwise. The native and impurity atoms are assumed to be exchanged with a reservoir at a chemical potential given by $\mu_{i}$. The chemical potentials for oxygen, nitrogen, hydrogen, and boron are computed from the Gibbs free energy from an $\mathrm{O}_{2}, \mathrm{~N}_{2}$, and $\mathrm{H}_{2}$ molecule in the gas phase and rhombohedral boron in the solid phase, respectively. The charge state $q$ is included explicitly in the last term and describes the cost of adding or removing electrons from a reservoir at a potential set by the Fermi energy $\epsilon_{F}$ referenced with respect to the energy of the valence band maximum $\epsilon_{v}$ of the bulk reference cell. In general, in charged supercell calculations, due to periodic boundary effects, the reference potentials in the bulk and the defective supercell structures are not equal and need to be aligned with a term $\Delta v[66]$. Additionally, an image-charge correction term $E_{\text {corr }}^{q}$ has to be added to account for spurious long-range Coulomb interactions between the defect charge, its periodic images, and the neutralizing background charge[65].

\subsubsection{X-RAY ABSORPTION SPECTROSCOPY}

X-ray absorption spectroscopy (XAS) was simulated within the density functional theory excited core-hole (DFT-XCH) approach[36] where the photo excited atom is modeled by removing a core electron from the pseudopotential and placing it in the first available empty state. The electronic structure problem of the system that now includes 
the core-hole is then solved self-consistently under constrained occupations while employing the Shirley interpolation scheme[49] to generate optimal basis sets in order to reduce computational cost. The absorption spectrum is computed by evaluating the transition probability between the initial and final state as given by Fermi's golden rule within the dipole approximation. The resulting spectrum is broadened uniformly with a Gaussian of $0.2 \mathrm{eV}$ at FWHM. To correct for the well-known underestimation of the band gap by the PBE functional, the energy scale is stretched uniformly by a factor of 1.04. Due to the lack of an absolute energy reference inherent in the pseudopotential method, an energy alignment scheme was employed to yield comparably meaningful relative energies for structurally and chemically different systems[67]. Finally, the entire spectrum is shifted by a single value, which is kept constant for all computed spectra, to align with the experimental data.

\subsection{RESULTS}

\subsubsection{STRUCTURE OF TRIANGULAR VOIDS}

To investigate the structural and thermodynamic stability of equilateral triangular defects in $h$-BN, we created periodic supercell models of defects with varying edge termination and size in a crystalline host. We considered defects in both bulk and surface slab supercells and defect sizes originating from just a single vacancy with three edge atoms up to a void created by removing 16 atoms, yielding 12 edge atoms in total. In addition to the experimentally observed N- and B-terminated edges, we also investigate the possibility of oxygen-terminated edges and defects with $\mathrm{N}$ - or B-terminated edges passivated with hydrogen atoms. Additionally, we considered N-terminated defects with passivated dangling bonds by electron doping instead of hydrogen. A schematic representation of a subset of the relaxed supercells used in the calculations is displayed in Fig. 3.1.

All but the O-terminated defects suffered from a large distortion of the crystal lattice, especially in the vicinity of the created triangular void for all considered sizes. The distortion of the crystal lattice after relaxing the O-terminated defects was minimal, with oxygen atoms displacing slightly away from the center of the void. In the case of B-terminated edges shown in Fig. $3.1(\mathrm{~d})$, the boron atoms at the edge of the void are undercoordinated and have a dangling bond which leads to the contraction of the void where the corners of the defect almost form a pentagon. Similar behavior is observed for the neutral N-terminated defects depicted in Fig. 3.2(a), but the deformation is even stronger compared to B-terminated defects.

By doping the N-terminated defect structures with additional electrons (one for each $\mathrm{N}$ edge atom) the dangling bonds are passivated and the triangular shape of the void is largely restored as seen in Fig. 3.2(c). Doping the structure with electrons does 

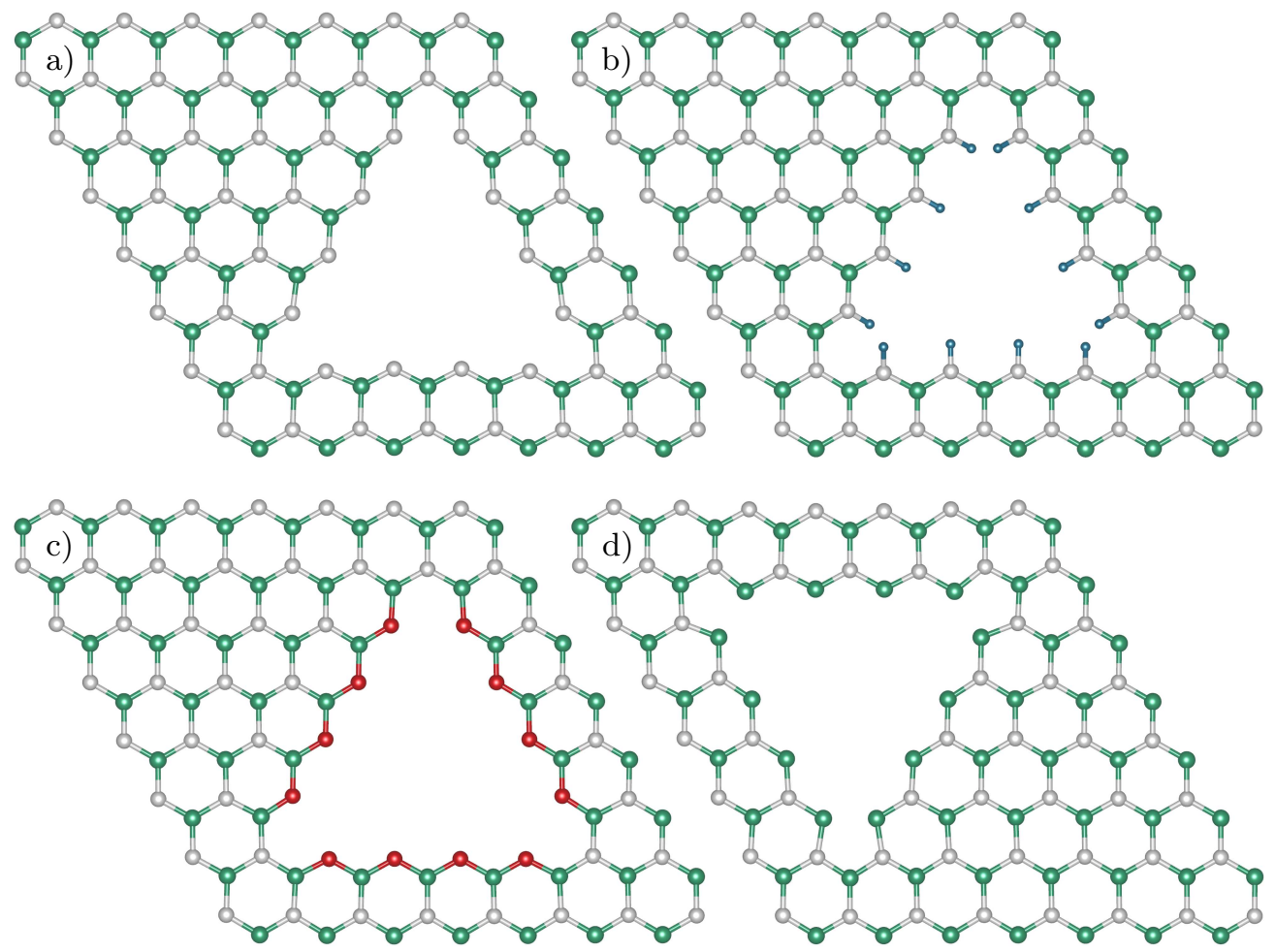

Figure 3.1: Partial images of $h$-BN crystalline supercell including triangular defect with four out of six different edge terminations investigated in this study: (a) electrondoped nitrogen-terminated $\mathrm{V}_{\mathrm{B}_{10} \mathrm{~N}_{6}}^{\mathrm{N}}$, (b) hydrogen-passivated nitrogen-terminated $\mathrm{V}_{\mathrm{B}_{10} \mathrm{~N}_{6}}^{\mathrm{N}: \mathrm{H}}$, (c) oxygen-terminated $\mathrm{V}_{\mathrm{B}_{10} \mathrm{~N}_{6}}^{\mathrm{O}}$ and $(\mathrm{d})$ boron-terminated $\mathrm{V}_{\mathrm{B}_{6} \mathrm{~N}_{10}}^{\mathrm{B}}$. The structures shown are all relaxed under neutral charge conditions with the exception of the nitrogen-terminated case, where one electron was added for each under coordinated edge atom. Hydrogen, nitrogen, boron and oxygen atoms are colored blue, gray, green, and red, respectively. 


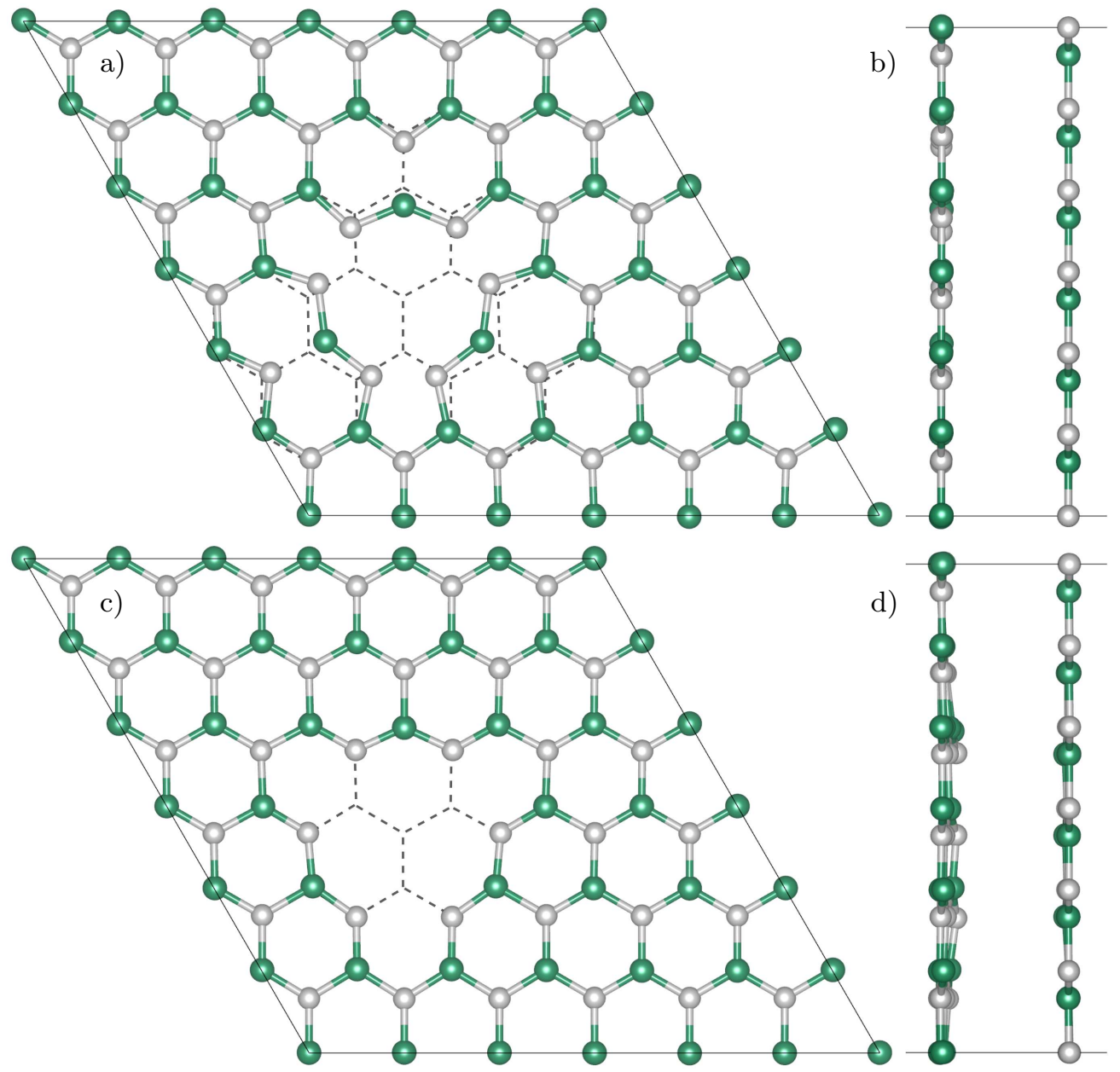

Figure 3.2: Top view of part of the surface plane of the $V_{\mathrm{B}_{3} \mathrm{~N}_{1}}^{N}$ supercell model for the (a) neutral and (c) charged supercell surface slab model. The side views (b) and (d) correspond to the top two layers of the neutrally and negatively charged slab, respectively, with the leftmost plane being the surface plane. The dashed lines indicate the unperturbed crystal lattice. 


\begin{tabular}{lcccc}
\hline & \multicolumn{2}{c}{ Boron:H } & \multicolumn{2}{c}{ Nitrogen:H } \\
\hline Defect size & Average & Max & Average & Max \\
\hline$V_{\mathrm{B}_{1}}$ & 1.09 & 1.30 & 0.69 & 1.02 \\
$V_{\mathrm{B}_{3} \mathrm{~N}_{1}}$ & 0.95 & 1.12 & 0.59 & 0.72 \\
$V_{\mathrm{B}_{6} \mathrm{~N}_{3}}$ & 0.64 & 1.00 & 0.49 & 0.77 \\
$V_{\mathrm{B}_{10} \mathrm{~N}_{6}}$ & 0.65 & 0.98 & 0.47 & 0.79 \\
\hline$V_{\mathrm{B}_{1}}$ & 1.08 & 1.09 & 0.83 & 1.35 \\
$V_{\mathrm{B}_{3} \mathrm{~N}_{1}}$ & 0.97 & 1.38 & 0.61 & 0.74 \\
\hline
\end{tabular}

TABle 3.1: Average and maximum vertical planar displacement in $\AA$ of hydrogen atoms in hydrogen passivated B- and N-terminated defects.

however result in the deformation of the planar symmetry as shown in Fig. 3.2(d). This planar deformation of the defective plane occurs only in the asymmetric surface slab calculation for a charged supercell and this effect was not observed in the neutral surface slab configuration as shown in Fig. 3.2 (b) nor in the charged bulk supercells. In the charged bulk supercells similar distorting effects are present but, mirrored symmetrically on both sides of the defective plane, these are canceled out.

Defect structures with a B- and N-terminated edge passivated by hydrogen also showed significant structural deformation around the defect void. The hydrogen atoms in $\mathrm{H}-$ passivated defects [see Fig. 3.1 (b) in the case of the passivated N-terminated edge] are all oriented perpendicular with respect to their parent edge and, along with the nitrogen or boron atoms they bond to, undergo heavy buckling out of the crystalline plane. The average and maximum planar displacement of hydrogen atoms in H-passivated B- and N-terminated edges are presented in Table 3.1.

For the smaller defects the vertical displacement can be as much as $1.38 \AA$, and as the defect grows the average displacement seems to reach an equilibrium value. This can be understood from the fact that at a vertex of a triangular defect two passivating hydrogens atoms are at closest proximity in plane and therefore have to displace further out of plane to maintain the appropriate distance from each other. For the smallest defect all three passivating hydrogen atoms are at the triangle's vertex, but for increasing defect size hydrogen atoms appear that are on the triangle side. The boron-terminated defects exhibited even stronger buckling compared to the N-terminated defects, which is reflected by the larger average displacement of hydrogen atoms.

\subsubsection{TRIANGULAR DEFECT FORMATION ENERGY}

The thermal stability of defects can be quantified by calculating the formation energies given by Eq. 5.1. Schemes to calculate the charge correction terms $E_{\text {corr }}^{q}$ and $q \Delta v$ only exist for point defects and not for the multidefect systems that we investigate in this 
work. Given that the electron-doped N-terminated defects are the only charged cells and that the charge corrections become less prominent for increasing supercell sizes and the supercells used for these calculations are quite large, it is reasonable to neglect these corrections as we have done in our calculations. Computed formation energies for $\epsilon_{F}=0$ in electronvolts per edge atom for the various defect structures are shown in Table 3.2. Note that the difference between surface and bulk formation energies is almost negligible within the accuracy of the calculations for almost all size and termination types. The most apparent conclusion that can be drawn instantly is that the O-terminated defects have the lowest formation energy and are therefore energetically most favorable for all defect sizes considered, which perfectly mirrors the minimal crystal distortion observed for O-terminated defects after relaxation as discussed in the previous section. The electron-doped N-terminated defects have the largest formation energies closely followed by the unpassivated N- and B-terminated edges. As described in the previous section, the added electrons reduce the in-plane deformation but also cause an interaction with neighboring planes which in the asymmetric surface case leads to planar deformation (see Fig. 3.2). An increased Fermi level will reduce the formation energy for the electron passivated N-terminated defects but a value of $7 \mathrm{eV}$ is required to approach the formation energies of the O-terminated defects which is larger than the material band gap and is highly unlikely to occur. Passivating the dangling bonds of the under coordinated edge atoms for B- and N-terminated defects with hydrogen atoms reduces the formation energy significantly but is still less favorable compared to the O-terminated defects. The difference in formation energies between hydrogen passivated and unpassivated defects is smaller for B-terminated defects compared to the N-terminated defects and can be explained by the fact that even though the addition of hydrogen passivates the dangling bonds and reduces the formation cost, this comes at the expense of planar deformation around the defect edge which is larger for B-terminated defects.

Defects that are created in situ during TEM measurements, due to the high-energy electron beam, are likely to be N-terminated and, in agreement with our results, have been observed to be unstable[52, 54]. Sample preparation methods that employ plasma to etch a multilayer down to a monolayer can introduce contaminants like oxygen into the sample prior to the measurement[53]. We find that, in contrast with the highly unstable N-terminated edges, O-terminated edges are very stable from a thermodynamic point of view and should be taken into account as a potential cause of triangular voids when studying defects in $h$-BN. To the best of our knowledge, O-terminated triangular voids in $h$-BN have not yet been considered in the TEM literature but, as mentioned in the Introduction, the presence of oxygen defects in bulk or thin film $h$-BN has been proposed in several x-ray absorption spectroscopy studies[58-60]. 


\begin{tabular}{lcccccc}
\hline & \multicolumn{6}{c}{ Edge termination and/or passivation } \\
\hline Defect size & $\mathrm{B}$ & $\mathrm{B}: \mathrm{H}$ & $\mathrm{N}$ & $\mathrm{N}: \mathrm{H}$ & $\mathrm{N}: \mathrm{e}$ & $\mathrm{O}$ \\
\hline$V_{\mathrm{B}_{1}}$ & 2.48 & 1.78 & 3.33 & 0.91 & 5.83 & -1.36 \\
$V_{\mathrm{B}_{3} \mathrm{~N}_{1}}$ & 2.26 & 1.79 & 3.26 & 1.13 & 6.23 & -1.02 \\
$V_{\mathrm{B}_{6} \mathrm{~N}_{3}}$ & 3.16 & 2.06 & 3.88 & 1.39 & 6.49 & -0.63 \\
$V_{\mathrm{B}_{10} \mathrm{~N}_{6}}$ & 3.81 & 2.38 & 4.39 & 1.76 & 6.59 & -0.23 \\
\hline$V_{\mathrm{B}_{1}}$ & 2.46 & 1.53 & 3.31 & 0.72 & 5.73 & -1.33 \\
$V_{\mathrm{B}_{3} \mathrm{~N}_{1}}$ & 2.36 & 1.77 & 3.41 & 1.11 & 6.49 & -0.95 \\
\hline
\end{tabular}

TABlE 3.2: Computed formation energies in electronvolts per edge atom, for triangular defects in $h$-BN for different sizes with boron (B), nitrogen (N) or oxygen (O) terminated edges. Formation energies of defects with boron- and nitrogen-terminated edges passivated by hydrogen are denoted by $\mathrm{B}: \mathrm{H}$ and $\mathrm{N}: \mathrm{H}$ respectively. The column marked N:e represents the formation energies of defects with nitrogen-terminated edges with one doped electron per edge atom. The upper half of the table are computed values for defects in the bulk supercell whereas the lower half of the table represents defect formation energies in the surface layer of a slab supercell. The Fermi level with respect to the valence band maximum in the bulk was set to $0 \mathrm{eV}$.

\subsubsection{X-RAY ABSORPTION SPECTROSCOPY OF HEXAGONAL BORON NITRIDE}

We have experimentally collected the boron $K$-edge absorption spectrum of a typical defective multilayer $h$-BN sample (see the Appendix for experimental details), displayed as a dotted black line in Figs. 3.3 (a) and 3.3 (b).

The main feature at $192 \mathrm{eV}$ is typical for pristine $h$-BN and originates from an excitonic state with $\pi^{*}$ character located in the band gap. A visual representation of the wavefunction corresponding to this state is plotted in Figs. 3.4 (a) and 3.4 (b) and its highly localized character explains the high intensity of the corresponding feature in the x-ray absorption spectrum. The three additional lower-intensity features on the high-energy side of the $\pi^{*}$ observed in the spectrum of the defective thin film are experimentally not observed for pristine $h$-BN[57]. We have computed the x-ray absorption spectrum within the density functional theory framework for pristine $h$-BN at a temperature of $0 \mathrm{~K}$ and $320 \mathrm{~K}$ [shown in Fig. 3.3 (a) and 3.3 (b) as the dashed gray and solid red line respectively] and the absence of the triplet of $\pi^{*}$ transitions in either spectrum confirms that these transitions can not be associated with pristine $h$-BN nor by structural changes induced by thermal effects. The relative intensity of the triplet of $\pi^{*}$ features with respect to the main $\pi^{*}$ transition has been shown to be positively correlated with the total oxygen content of the film and therefore it has been hypothesized that the satellite peaks originate from boron atoms whose local $\mathrm{BN}_{3}$ coordination is altered by the substitution of up to three nitrogen atoms with oxygen atoms, where the higher energy peak corresponds to a boron atom coordinated to three oxygen atoms[58-60]. 

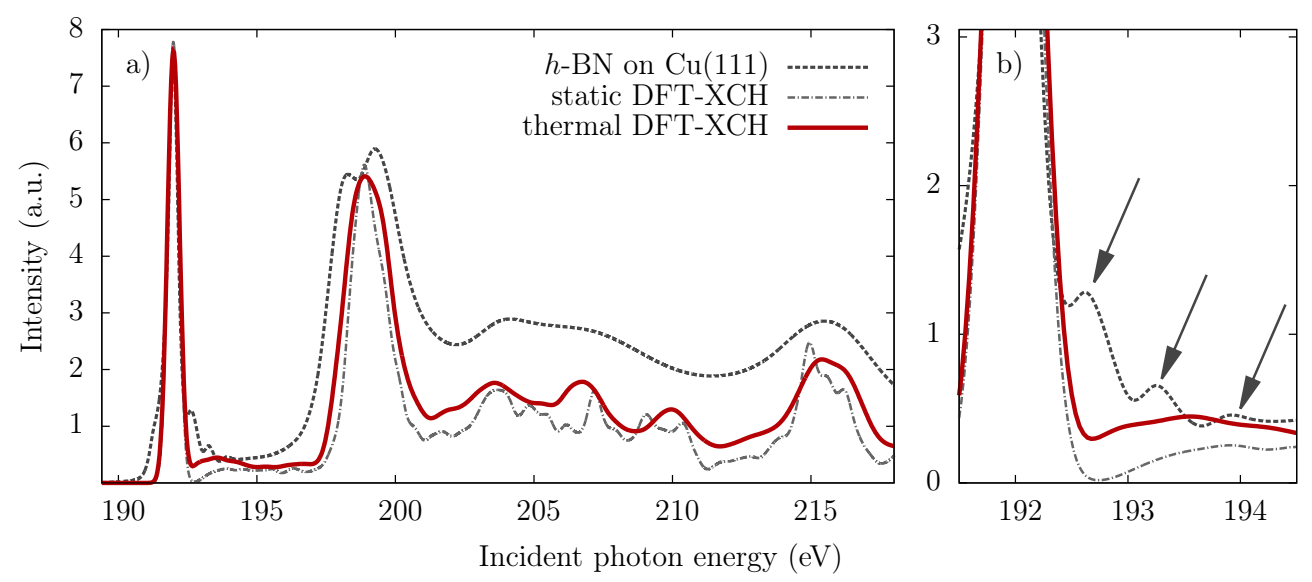

Figure 3.3: (a) Measured and simulated x-ray absorption spectrum of the boron $K$-edge in $h$-BN. The dotted black line is the measured spectrum for the $13 \mathrm{~nm}$ thin-film on the $\mathrm{Cu}(111)$ substrate. The simulated spectra for the static structure at a temperature of $0 \mathrm{~K}$ and the thermally equilibrated structure are shown in a dashed gray and solid red line respectively.

(b) Close up of main $\pi^{*}$ and three satellite features indicated by arrows.

a)

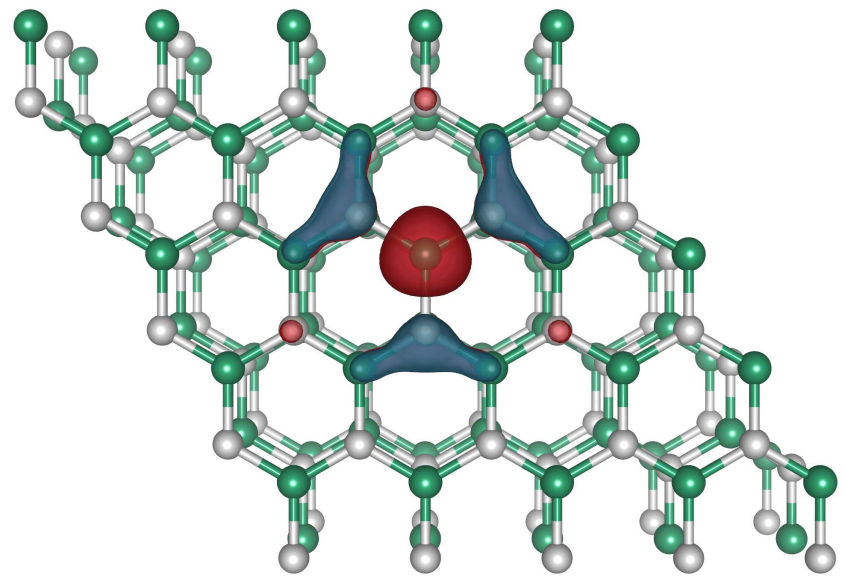

b)

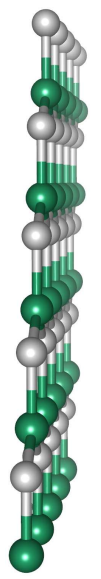

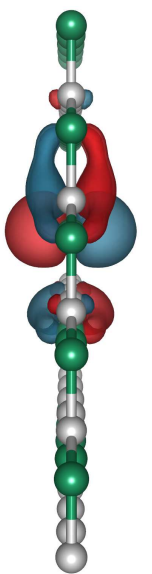

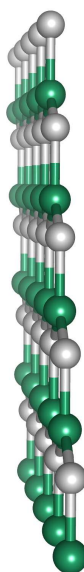

Figure 3.4: (a) Top and (b) side view of a $5 \times 5 \times 1 h$-BN supercell with an isosurface representation of the squared wavefunction $|\psi(\vec{r})|^{2}$ of the excitonic state corresponding to the intense transition in the B $K$-edge spectrum at $192 \mathrm{eV}$. The excitonic state is highly localized and has distinct antibonding $p$-like character. 
A rigorous theoretical study on the direct effect of substitutional oxygen defects on the x-ray absorption spectrum of $h$-BN, however, has not yet been carried out.

\subsubsection{OXYGEN DEFECTS FORM TRIANGULAR VOIDS}

To verify the proposed origin of the $\pi^{*}$ satellites as a result of oxygen defects, we first need to determine the structural model with which oxygen atoms are incorporated in the hexagonal boron nitride crystal lattice. Schematic representations of the three increasingly oxygen coordinated environments are shown in Figs. 3.5 (a) and 3.5 (c) which henceforth are referred to as $\mathrm{BN}_{2} \mathrm{O}, \mathrm{BNO}_{2}$ and $\mathrm{BO}_{3}$ respectively. Creating any one of these $\mathrm{BN}_{3-x} \mathrm{O}_{x}(1 \leq x \leq 3)$ defect environments in a crystalline $h$-BN supercell, quickly reveals an issue with this structural model, proposed in previous experimental literature[58-60]. By simply replacing nitrogen with oxygen atoms, the oxygen atoms in the final defect structure will be over coordinated. The structure of the most stable crystalline boron oxide $\mathrm{B}_{2} \mathrm{O}_{3}$ reveals that unlike the trigonally bonded boron and nitrogen in $h$-BN, a twofold coordination is more favorable for oxygen.

Attempts to relax the defect structures with trigonally coordinated oxygen atoms resulted in a strong distortion of the planar symmetry and for $\mathrm{BO}_{3}$ the central boron atom was fully ejected out of the plane. This strongly reflects the tendency for the oxygen atoms to attain twofold coordination. To construct substitutional oxygen defects that yield $\mathrm{BN}_{3-x} \mathrm{O}_{x}$ local environments within the crystalline $h$-BN host, additional vacancies have to be created to ensure the twofold coordination of the oxygen atoms. Defective supercell structures that support each of the three local environments $\mathrm{BN}_{2} \mathrm{O}$, $\mathrm{BNO}_{2}$ and $\mathrm{BO}_{3}$ are shown in Figs. $3.5(\mathrm{~d})$ and $3.5(\mathrm{f})$, respectively. It is crucial to realize that this immediately reveals the intrinsic connection between oxygen defects and triangular voids in $h$-BN, as they form in parallel, directly as a result of the preferred coordination difference between oxygen and nitrogen.

\subsubsection{X-RAY ABSORPTION SPECTROSCOPY OF OXYGEN DEFECTS}

Computed x-ray absorption spectra for excited boron atoms in each of the four possible oxygen coordination environments are shown in Fig. 3.6. The four features observed in the experimental spectrum, labeled $\pi_{0}^{*}, \pi_{1}^{*}, \pi_{2}^{*}$ and $\pi_{3}^{*}$ are hypothesized to correspond to excitations from boron atoms that have a local $\mathrm{BN}_{3}, \mathrm{BN}_{2} \mathrm{O}, \mathrm{BNO}_{2}$ and $\mathrm{BO}_{3}$ local environment, respectively.

In agreement with the hypothesis, the simulated spectra show an increasing blue shift of the main absorption feature for an increasing oxygen coordination of the boron atom. The blue shift arises due to two competing processes. Oxygen is more electronegative compared to nitrogen and so the partial charge on a boron atom will become more positive for an increasing oxygen coordination. An increase in the partial positive 

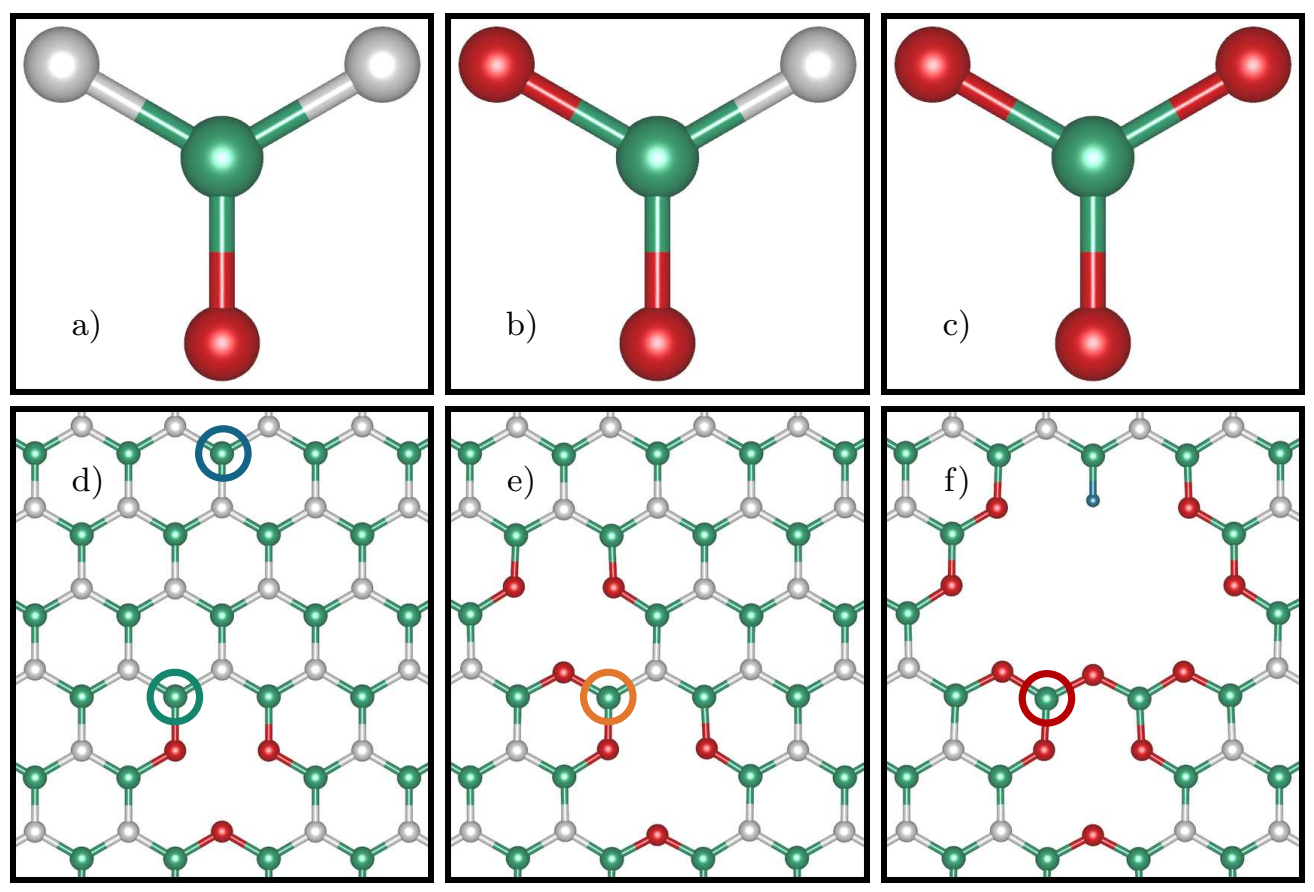

Figure 3.5: $(\mathrm{a}-\mathrm{c})$ Schematic representation of the $\mathrm{BN}_{3-x} \mathrm{O}_{x}(1 \leq x \leq 3)$ substitutional oxygen defect environments of boron atoms in $h$-BN. (d-f) Defect structures in a $h$-BN host crystal, that hosts a boron atom with $\mathrm{BN}_{2} \mathrm{O}, \mathrm{BNO}_{2}$ and $\mathrm{BO}_{3}$ environment respectively, while maintaining twofold coordination for the oxygen atoms and threefold coordination for nitrogen and boron atoms. The colors of the circles around selected boron atoms correspond to spectra in Fig. 3.6. 


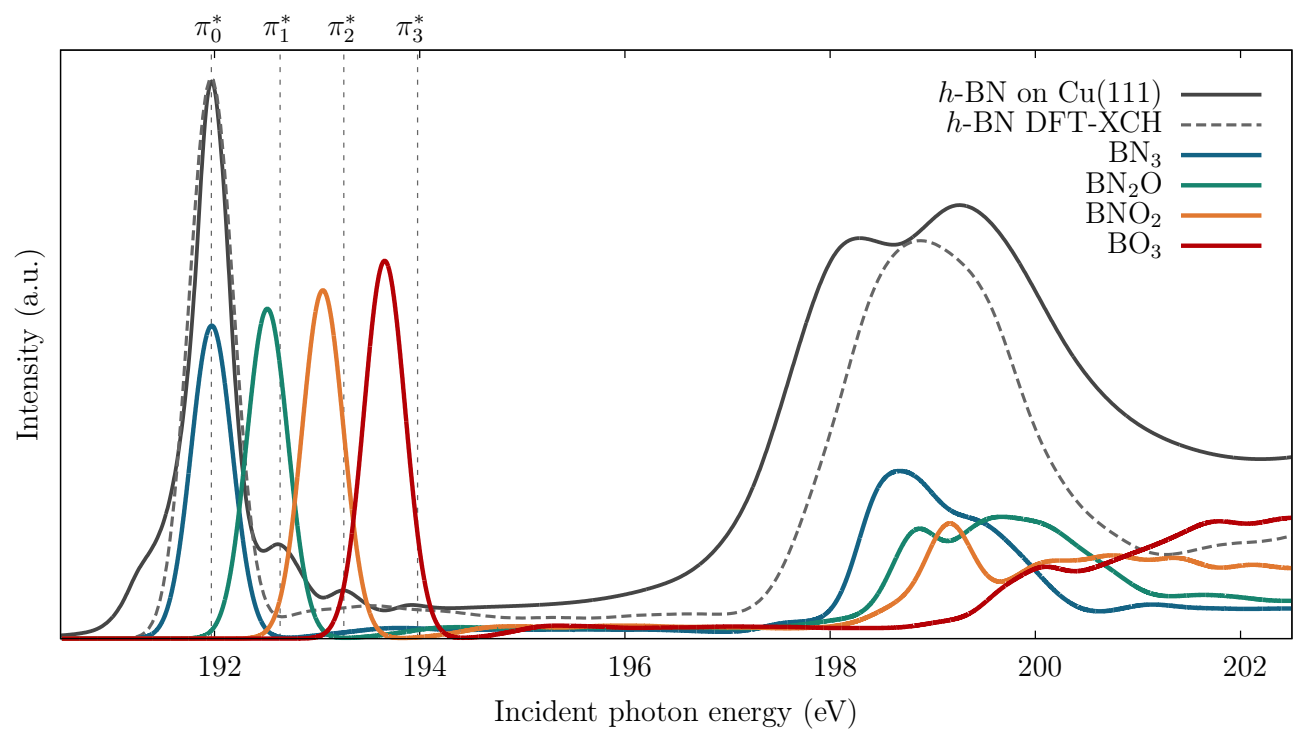

Figure 3.6: Measured and simulated B $K$-edge x-ray absorption spectra for hexagonal boron nitride. The solid black line corresponds to the measured spectrum for multilayer $h$-BN on a $\mathrm{Cu}(111)$ substrate and the dashed gray line is the thermally averaged simulation. The four colored spectra with the first peak increasing from lower to higher energy, represent the simulated spectrum of a single boron atom with a local $\mathrm{BN}_{3}, \mathrm{BN}_{2} \mathrm{O}, \mathrm{BNO}_{2}$ and $\mathrm{BO}_{3}$ environment, respectively. The labels $\pi_{0}^{*}-\pi_{3}^{*}$ and corresponding vertical dashed lines denote the energy position of the main $\pi^{*}$ and satellite peaks as observed in experiment. 


\begin{tabular}{lcccc}
\hline & \multicolumn{4}{c}{ Local environment of excited atom } \\
\hline & $\mathrm{BN}_{3}$ & $\mathrm{BN}_{2} \mathrm{O}$ & $\mathrm{BNO}_{2}$ & $\mathrm{BO}_{3}$ \\
\hline$h$-BN & +2.21 & - & - & - \\
Cluster 1 & +2.21 & +2.24 & - & - \\
Cluster 2 & +2.21 & +2.25 & +2.30 & - \\
Cluster 3 & +2.21 & +2.25 & +2.30 & +2.34 \\
\hline
\end{tabular}

TABLE 3.3: Average partial charges for core-hole excited boron atoms with varying local configuration in bulk $h$-BN and the three structures with substitutional oxygen defects. Clusters 1 through 3 correspond to supercell structures as depicted in Figs. 3.5 (d) and 3.5 (f), respectively.

charge will reduce the possible screening of core-excitations, thereby deepening the core-level binding energies, effectively increasing the energy separation between the $1 \mathrm{~s}$ level and the first available empty state into which the core electron is excited. The oxygen defects also influence the density of states of the material, changing the relative energy separation of the unoccupied orbitals with respect to the core states. Unlike the screening of core states effect, this change in the unoccupied density of states does not necessarily have to result in a blue shift but could also cause a red shift. It is important to note that the character of the excitonic state does not change as the oxygen coordination of the excited boron atom changes and remains a highly localized state with anti-bonding $p$-character. The electron density is mostly located on the excited atom itself and the bonds between its first and second nearest neighbors [see Figs. 3.4 (a) and 3.4(b)]. These bonds are broken when oxygen atoms are introduced and effectively the excitonic state becomes even more localized on the remaining bonds. This increased localization also increases the overlap with the $1 s$ core state which explains the increase in intensity of the $\pi^{*}$ feature for an increasing oxygen coordination.

\subsection{DISCUSSION}

To investigate the effect of the deepening of the core-levels we have computed the local electron population on the boron atoms through Bader analysis[68] for the various possible oxygen coordinations as shown in Table 3.3.

The Bader charge analysis confirms that indeed for an increasing oxygen coordination, the partial charge on the boron atom becomes more positive. As described before, this reduces the screening of core electrons which increases the energy separation between the $1 s$ core-level state and the lowest unoccupied molecular orbital (LUMO), resulting in a blue shift of the $\pi^{*}$ feature in the XAS. The dependency between the oxygen coordination of the boron atom and its computed Bader charge is perfectly linear within 
the accuracy of the calculations. The same linear dependence is observed in the position of the $\pi^{*}$ transitions as a function of oxygen coordination, both in experiment and simulation, with the exception of the highest energy satellite peak in experiment. The measured energy separations between the first three peaks are very consistent at $0.63 \mathrm{eV}$ but the separation between the two peaks highest in energy is significantly higher at $0.75 \mathrm{eV}$, in agreement with previous experimental work[57, 58,60]. It is interesting to note that the increase in energy separation for the two higher-energy peaks is not observed in our simulations and a potential cause might be that boron atoms with $\mathrm{BO}_{3}$ coordination actually lose their planar geometry inherited from the $h$-BN crystal structure and obtain a more three-dimensional orientation reminiscent of the crystalline structure found in $\mathrm{B}_{2} \mathrm{O}_{3}$. When a significant $\mathrm{BO}_{3}$ peak is observed, the corresponding amount oxygen is more likely to be located in small $\mathrm{B}_{2} \mathrm{O}_{3}$ clusters rather than as substitutional defects in the $h$-BN lattice. X-ray absorption simulations of the crystalline $\mathrm{B}_{2} \mathrm{O}_{3}$ system (not shown) indeed show a $\pi^{*}$ transition at $194.0 \mathrm{eV}$ which overlaps exactly with observations from experiment $[58,60]$. The remaining underestimation of the computed blue shift can be attributed to the fact that the energy alignment scheme does not account for self-interaction errors introduced by the quasiparticle approach. The computed spectra are aligned by comparing the energy of the ground state to the excited system with the core-hole and the self-interaction energy of the photo excited electron is not corrected for.

\subsection{CONCLUSIONS}

In conclusion, we have presented a theoretical estimate of the thermodynamic stability of triangular defects in $h$-BN as observed in multiple TEM studies for various edge terminations. Nitrogen-terminated defects are highly unstable in agreement with experimental TEM literature and are slightly stabilized by injecting additional electrons to passivate any dangling bonds. Oxygen-terminated edges are found to be the most stable edge termination of the types considered and we suggest that there is a large probability for the existence of stable triangular defects with O-terminated edges in $h$ BN. It should be noted that this does not necessarily imply that defects investigated in the experimental TEM literature as discussed in this work were in fact O-terminated. As these defects are created in-situ under the influence of the high energy electron beam during the measurement, they are most likely natively terminated by boron or nitrogen. However this work does show that any work that studies defects in $h$-BN should consider the presence of oxygen defects as a significant possibility.

Additionally, we provide first-principles calculations of boron $K$-edge x-ray absorption spectra to confirm the hypothesis that a commonly observed triplet of $\pi^{*}$ transitions in the experimental XAS of defective $h$-BN films is due to substitutional oxygen defects that decorate the excited boron atom[58-60]. As a photo excited boron atom is increasingly oxygen coordinated its core-levels deepen and the strong $\pi^{*}$ excitonic 
transition is increasingly blue shifted. The structural model that is solely capable of explaining the structural stability and the additional defect features observed in the spectroscopy is that of oxygen atoms replacing nitrogen atoms in the hexagonal crystal lattice with the stringent constraint that the oxygen atoms are twofold coordinated. The difference between the twofold coordination of the substitutional oxygen and the threefold coordination of the boron and nitrogen directly causes the formation of equilateral shaped voids in the direct vicinity of the oxygen defects. These simulations provide indirect proof that any $h$-BN film that exhibits the triplet of $\pi^{*}$ features in the boron $K$-edge will have triangular voids with O-terminated defects. This can be verified by a STEM-EELS experiment that actively looks for the predicted spectral signature in the boron $K$-edge spectrum.

Insights into the nature of defects in $h$-BN as presented in this work will prove valuable in future work that aims to minimize defect concentrations. Additionally, the predicted exceptional stability of O-terminated triangular defects may enable novel applications of purposefully created defects as in, for example, nanosieves.

\section{ACKNOWLEDGMENTS}

This work is supported by NanoNextNL, a micro and nanotechnology programme of the Dutch Government and 130 partners. We acknowledge the support of the Center for X-ray Optics of Lawrence Berkeley Laboratory and the Industrial Focus Group XUV Optics at the MESA+ Institute for Nanotechnology at the University of Twente, notably the partners ASML, Carl Zeiss SMT GmbH, and the Foundation FOM. All the computational work was performed at the Molecular Foundry which is supported by the Office of Science, Office of Basic Energy Sciences, of the United States Department of Energy under Contract No. DE-AC02-05CH11231. 


\section{REFERENCES}

[8] C. Zhi, Y. Bando, C. Tang, H. Kuwahara, and D. Golberg, Adv. Mater. 21, 2889 (2009).

[9] L. Song, L. Ci, H. Lu, P. B. Sorokin, C. Jin, J. Ni, A. G. Kvashnin, D. G. Kvashnin, J. Lou, B. I. Yakobson, and P. M. Ajayan, Nano Letters 10, 3209 (2010).

[10] Y. Chen, J. Zou, S. J. Campbell, and G. Le Caer, Applied Physics Letters 84, 2430 (2004).

[11] A. Zunger, A. Katzir, and A. Halperin, Phys. Rev. B 13, 5560 (1976).

[12] K. K. Kim, A. Hsu, X. Jia, S. M. Kim, Y. Shi, M. Hofmann, D. Nezich, J. F. Rodriguez-Nieva, M. Dresselhaus, T. Palacios, and J. Kong, Nano Letters 12, 161 (2012).

[13] C. R. Dean, A. F. Young, I. Meric, C. Lee, L. Wang, S. Sorgenfrei, K. Watanabe, T. Taniguchi, P. Kim, K. L. Shepard, and J. Hone, Nature Nanotech 5, 722 (2010).

[14] J. C. Meyer, A. Chuvilin, G. Algara-Siller, J. Biskupek, and U. Kaiser, Nano Letters 9, 2683 (2009).

[36] D. Prendergast, and G. Galli, Phys. Rev. Lett. 96, 215502 (2006).

[42] J. Underwood, and E. Gullikson, Journal of Electron Spectroscopy and Related Phenomena 92, 265 (1998).

[43] E. M. Gullikson, S. Mrowka, and B. B. Kaufmann, Recent developments in euv reflectometry at the advanced light source, 2001.

[47] G. Kresse, and J. Furthmüller, Physical Review B 54, 11169 (1996).

[49] E. L. Shirley, Phys. Rev. B 54, 16464 (1996).

[50] K. Watanabe, T. Taniguchi, and H. Kanda, Nat Mater 3, 404 (2004). 
[51] K. K. Kim, A. Hsu, X. Jia, S. M. Kim, Y. Shi, M. Dresselhaus, T. Palacios, and J. Kong, ACS Nano 6, 8583 (2012).

[52] C. Jin, F. Lin, K. Suenaga, and S. Iijima, Phys. Rev. Lett. 102, 195505 (2009).

[53] N. Alem, R. Erni, C. Kisielowski, M. D. Rossell, W. Gannett, and A. Zettl, Phys. Rev. B 80, 155425 (2009).

[54] K. Suenaga, H. Kobayashi, and M. Koshino, Phys. Rev. Lett. 108, 075501 (2012).

[55] O. Cretu, Y.-C. Lin, M. Koshino, L. H. G. Tizei, Z. Liu, and K. Suenaga, Phys. Rev. Lett. 114, 075502 (2015).

[56] S. Azevedo, J. R. Kaschny, C. M. C. de Castilho, and F. de Brito Mota, Nanotechnology 18, 495707 (2007).

[57] I. Jiménez, A. F. Jankowski, L. J. Terminello, D. G. J. Sutherland, J. A. Carlisle, G. L. Doll, W. M. Tong, D. K. Shuh, and F. J. Himpsel, Phys. Rev. B 55, 12025 (1997).

[58] M. Niibe, K. Miyamoto, T. Mitamura, and K. Mochiji, Journal of Vacuum Science \& Technology A 28, 1157 (2010).

[59] I. Caretti, and I. Jiménez, Journal of Applied Physics 110, 023511, (2011).

[60] K. Simonov, N. Vinogradov, M. Ng, A. Vinogradov, N. Mårtensson, and A. Preobrajenski, Surface Science 606, 564 (2012).

[61] P. E. Blöchl, Physical Review B 50, 17953 (1994).

[62] G. Kresse, and D. Joubert, Physical Review B 59, 1758 (1999).

[63] J. P. Perdew, K. Burke, and M. Ernzerhof, Phys. Rev. Lett. 77, 3865 (1996).

[64] A. Tkatchenko, and M. Scheffler, Phys. Rev. Lett. 102, 073005 (2009).

[65] H.-P. Komsa, T. T. Rantala, and A. Pasquarello, Physical Review B 86, 045112 (2012).

[66] C. G. V. de Walle, J. Appl. Phys. 95, 3851 (2004).

[67] A. H. England, A. M. Duffin, C. P. Schwartz, J. S. Uejio, D. Prendergast, and R. J. Saykally, Chemical Physics Letters 514, 187 (2011).

[68] W. Tang, E. Sanville, and G. Henkelman, Journal of Physics: Condensed Matter 21, 084204 (2009). 

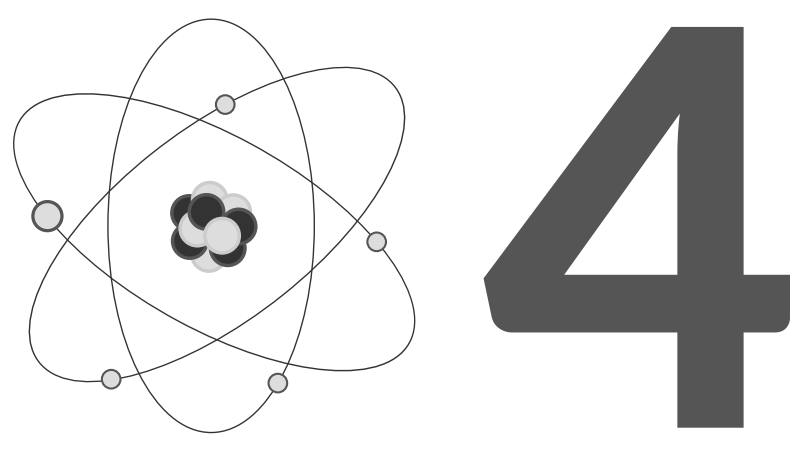

SELF-HEALING IN $B_{12} \mathrm{P}_{2}$ THROUGH MEDIATED DEFECT RECOMBINATION 


\section{ABSTRACT}

The icosahedral boride $\mathrm{B}_{12} \mathrm{P}_{2}$ has been reported to exhibit "self-healing" properties, after transmission electron microscopy recordings of sample surfaces, that were exposed to highly energetic particle beams, revealed little to no damage. In this work, employing calculations from first-principles within the density functional theory (DFT) framework, the structural characteristics of boron interstitial and vacancy defects in $\mathrm{B}_{12} \mathrm{P}_{2}$ are investigated. Using nudged elastic band simulations, the diffusion properties of interstitial and vacancy defects and their combination, in the form of Frenkel defect pairs, are studied. We find that boron icosahedra maintain their structural integrity even when in a degraded state in the presence of a vacancy or interstitial defect and that the diffusion activation energy for the recombination of an interstitial vacany pair, can be as low as $3 \mathrm{meV}$, in line with the previously reported observation of "self-healing". 


\subsection{INTRODUCTION}

The semiconductor $\mathrm{B}_{12} \mathrm{P}_{2}$, also referred to as icosahedral boron phosphide, is part of a group of boron-rich solids that form a class of superhard materials [69, 70] that, at their core, largely share the crystalline structure of $\alpha$-rhombohedral boron. The fundamental building block of these borides, is a structural unit of 12 boron atoms, whose crystallographic sites are defined by the 12 vertices of a convex regular icosahedron. The simplest crystalline boron allotrope, $\alpha$-rhombohedral boron, is formed by an orthorhombic arrangement of these boron icosahedra. Icosahedral boron phosphide, is one of the many boron-rich solids that is a direct derivative of $\alpha$-rhombohedral boron. In these solids, the icosahedral network is interspersed by dimers of phosphorus, arsenic or oxygen atoms, as in $\mathrm{B}_{12} \mathrm{P}_{2}, \mathrm{~B}_{12} \mathrm{As}_{2}$ or $\mathrm{B}_{12} \mathrm{O}_{2}$ respectively, or trimers of a combination of boron and carbon atoms, as in $\mathrm{B}_{4} \mathrm{C}$ and $\mathrm{B}_{13} \mathrm{C}_{2}$.

Due to their strongly related crystal structures, with the boron icosahedron at its foundation, these boron-rich materials share many interesting structural properties such as a high melting temperature and radiation hardness, making them ideal candidates for radiation resistant applications. In particular, the borides with pnictide inter-icosahedral dimers, $\mathrm{B}_{12} \mathrm{P}_{2}$ and $\mathrm{B}_{12} \mathrm{As}_{2}$, have been found to be extremely resistant to damage induced by high energy particle radiation[15]. Samples were irradiated by highly energetic electrons for several hours at room and lowered temperatures, however, post-irradiation transmission electron microscopy measurements of the sample surfaces revealed little to no signs of structural degradation. Under the conditions of the experiment, it was estimated that each boron atom, on average, was displaced at least several times for the duration of the exposure. A schematic depiction of a boron atom displacement as a result of a collision with a high energy particle is displayed in Fig. 4.1. Combined with the observed lack of structural damage, the conclusion was drawn that there must be a mechanism of "self-healing", that restores damage caused by the displacement of boron atoms and is active even in low thermal energy systems.

The high radiation hardness, as a result of this "self-healing" mechanism, makes these materials interesting for a variety of applications such as beta-voltaic devices[16] and, combined with the relatively high neutron capturing cross-section of boron, neutron detectors[17, 18]. However, to the best of the authors' knowledge, there has been no further experimental or theoretical research on the underlying mechanisms that give rise to this extraordinary "self-healing" property. The most obvious suggestion is that any boron vacancy and interstitial defect pair, commonly referred to as a Frenkel pair or Frenkel defect, created by a high energy collision, has both a high energy cost for creation and a low activation energy for recombination.

In this work, we will discuss the diffusion behavior of boron vacancy and interstitial defects, and combinations of the two to form Frenkel defect pairs, from first principles within the density functional theory (DFT) framework. Our results will show that 


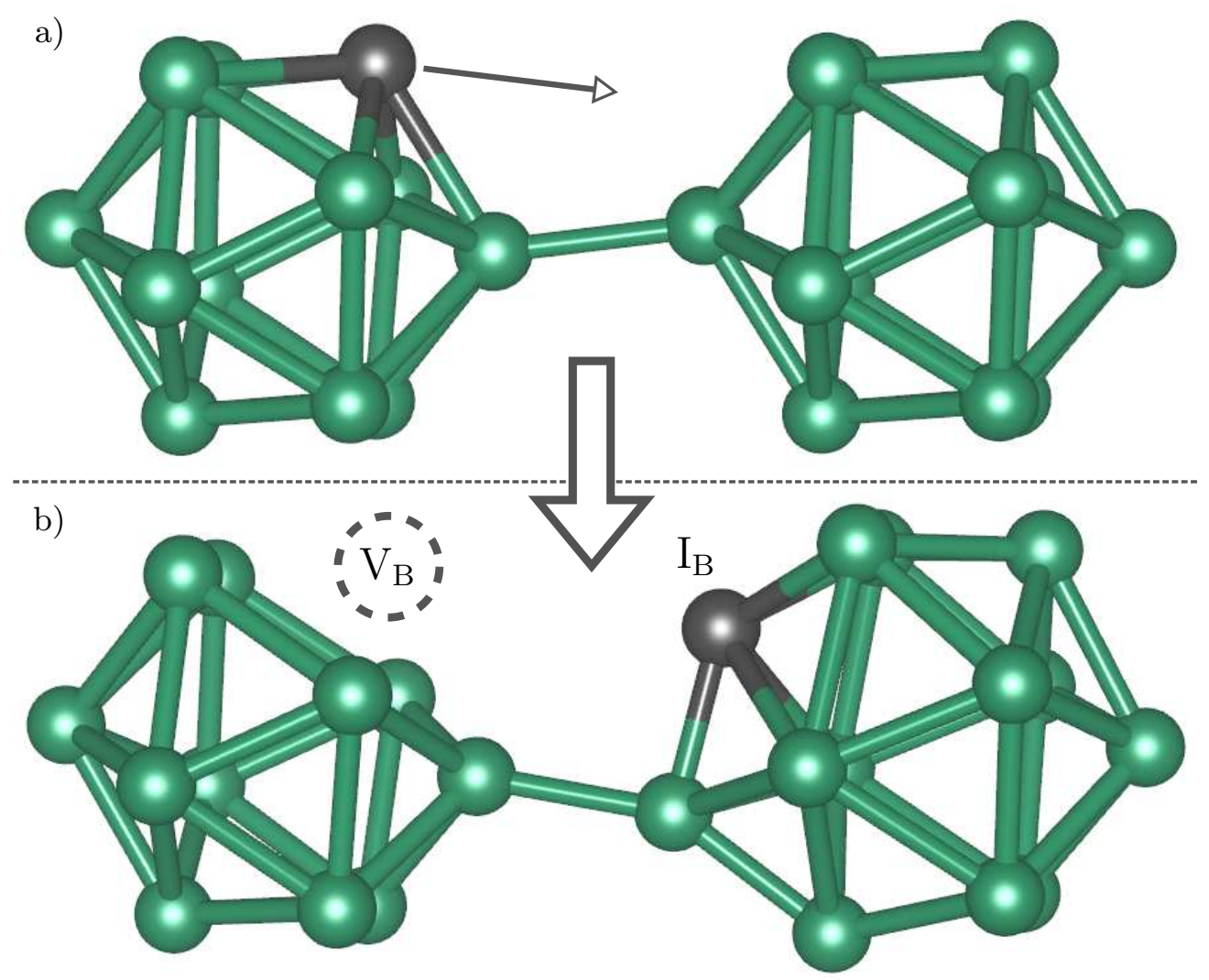

FiguRE 4.1: Schematic representation of boron Frenkel defect creation in an icosahedral boride. The top panel (a) represents two unperturbed boron icosahedra where the boron atom marked by a black sphere just received a large momentum in the direction of the arrow, transferred by a high energy particle in an inelastic scattering event. The bottom panel (b) shows the situation after the inelastic scattering event where a boron atom is displaced, forming an interstitial defect $I_{B}$ while leaving a vacancy defect $V_{B}$ behind. 
boron icosahedra are highly resilient atomic building blocks and maintain structural integrity even when in a degraded state in the presence of either a boron vacancy or interstitial defect. Employing nudged elastic band (NEB) calculations, we have investigated potential intra-icosahedral diffusion paths of vacancy and interstitial defects and calculated the diffusion activation energies. Combining a vacancy and interstitial defect into a Frenkel defect, NEB simulations are employed to determine potential recombination pathways, which will reveal that activation energies for the recombination of the Frenkel defect, separated on different icosahedra, can be as low as $3 \mathrm{meV}$, potentially explaining the experimentally observed radiation hardness.

\subsection{COMPUTATIONAL METHOD}

\subsubsection{STRUCTURAL RELAXATION}

All structural optimizations have been carried out within the DFT framework using the Vienna $a b$ initio simulation package VASP[47]. Core electrons are replaced by ultrasoft pseudopotentials within the projector augmented wave (PAW) method[61, 62] where the $2 s$ and $2 p$ electrons for boron and the $3 s$ and $3 p$ electrons for phosphorus are treated as valence electrons. The generalized gradient approximation (GGA) as formulated by Perdew-Burke-Ernzerhof (PBE) [63] is employed for the exchange-correlation energy. A $2 \times 2 \times 2$ supercell from the $\mathrm{B}_{12} \mathrm{P}_{2}$ unitcell was used for all calculations, totalling 288 boron and 48 phosphorus atoms for the perfect crystalline supercell. Plane waves were expanded up to an energy of $400 \mathrm{eV}$ and the Brillouin zone was sampled at the $\Gamma$ point, unless stated otherwise. All structures, with and without structural defects, were optimized by minimizing the Hellmann-Feynman forces acting on the nuclei below the threshold value $0.02 \mathrm{eV}^{-1}$.

\subsubsection{NUDGED ELASTIC BAND}

Nudged elastic band (NEB) calculations employed the climbing image method in combination with an improved tangent definition[71, 72], implemented as an extension of the previously mentioned software package VASP. Initial and final images for any NEB calculation were relaxed until all atomic forces were below the threshold value of $0.02 \mathrm{eV}^{-1}$. The initial guess for the NEB pathway was generated from the relaxed initial and final image, by linearly interpolating the atomic positions, where all resulting images are equidistant in terms of their relative Cartesian distance, unless explicitly stated otherwise. The relaxation threshold value, during the relaxation of all the images along the pathway under constraint of the imposed elastic band, was increased to $0.03 \mathrm{eV} \AA^{-1}$. During the relaxation process, the Brillouin zone was sampled at the $\Gamma$ point, but to compute the total energies of the relaxed images, the $k$-point density 
was increased to a $2 \times 2 \times 1$ Monkhorst-Pack mesh[73], in order to ensure convergence better than $2 \times 10^{-2} \mathrm{meV}$ per atom.

\subsubsection{MOLECULAR DYNAMICS}

All molecular dynamics have been carried out with VASP with identical values for the relevant DFT parameters described in section 4.2 .1 on the method of structural relaxation. For the simulation of the dynamics of Frenkel defect recombination, a $2 \times 2 \times$ $2 \mathrm{~B}_{12} \mathrm{P}_{2}$ supercell including a single boron interstitial of type $\mathrm{I}_{\mathrm{B}}^{\mathrm{A}}$, was first equilibrated at the target temperature by coupling the system to a Nosé-Hoover thermostat and sampling the canonical ensemble $(N V T)$. The time step was set to $0.2 \mathrm{fs}$. After an equilibration period of $3 \mathrm{ps}$, the thermostat was decoupled and the microcanonical ensemble $(N V E)$ was sampled for another 2 ps. From that point in time, in intervals of $1 \mathrm{ps}$, a vacancy was introduced at positions described in greater detail in the main text, and the system, that now includes a full Frenkel defect, was sampled for 1 ps.

\subsubsection{CHARGED DEFECTS}

Periodic boundary conditions provide an effective method of simulating pristine crystals within density functional theory. However, this approach has the downside that it introduces spurious interactions between local aperiodicities, such as structural defects, and their periodic images, unless the simulated cell is sufficiently large to render these interactions negligible. Additionally, structural defects in solid state materials can exist in various charge states and the long-ranged Coulomb interaction between the localized charge density and its periodic image can lead to significant inaccuracies in computed energies[65]. Correcting for these spurious interactions is non-trivial and several methods have been proposed[74-78], however no single rigorous correction method is currently available. This work will also consider charged defects but the calculated total energies will not explicitly be corrected for the fictitious charge induced terms. However, since only energy differences between systems with an identical charge state are used, the introduced error cancels out and the omission of an explicit correction is justified.

\subsection{RESULTS}

\subsubsection{CRYSTAL STRUCTURE}

A schematic representation of the crystal structure of $\mathrm{B}_{12} \mathrm{P}_{2}$ is shown Fig. 5.2. In this work, we consider the hexagonal unit cell, which contains 36 boron and 6 phosphorus atoms. The optimized lattice parameters $a$ and $c$ are $5.998 \mathrm{~nm}$ and $11.843 \mathrm{~nm}$, 

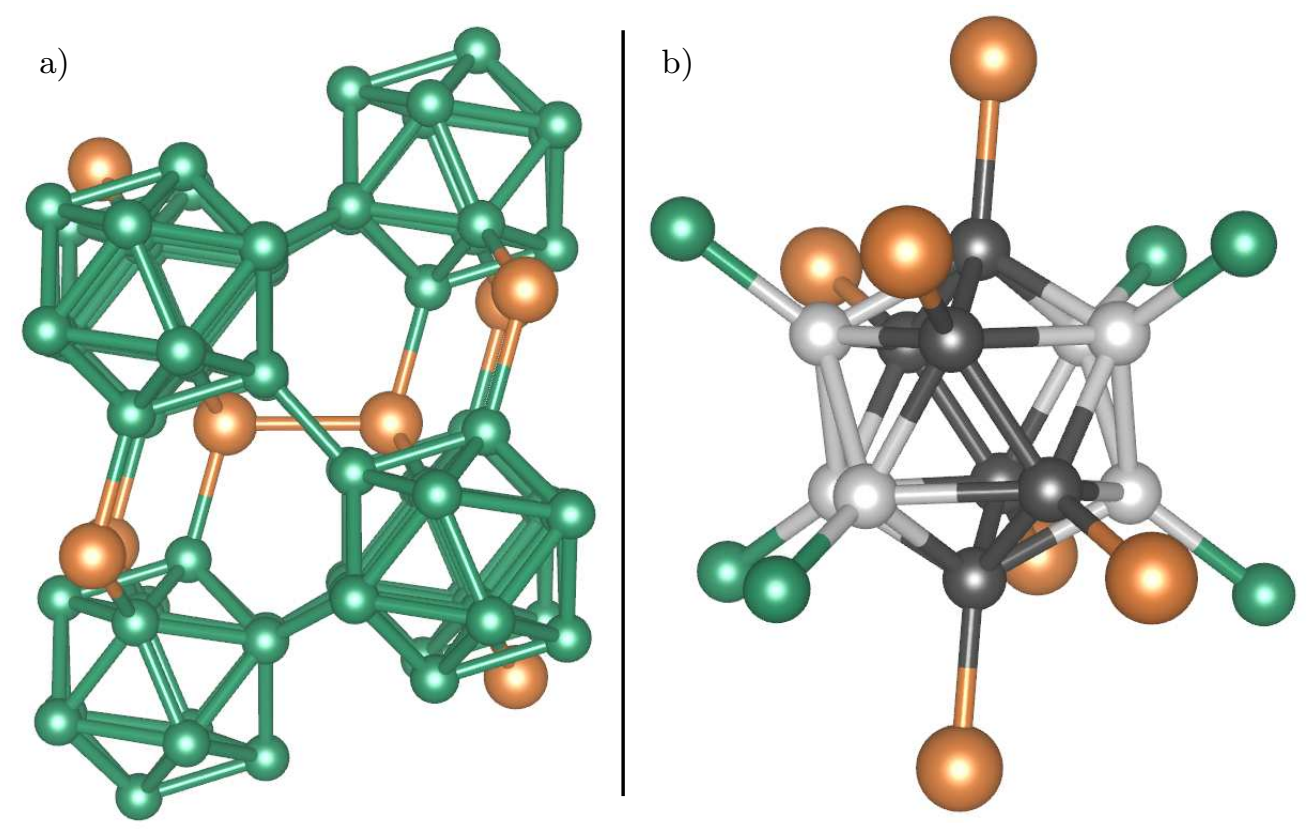

Figure 4.2: (a) A schematic depiction of the crystal structure of $\mathrm{B}_{12} \mathrm{P}_{2}$, where orange spheres represent phosphorus atoms and green spheres represent boron atoms. This depiction shows six complete boron icosahedra in an orthorhombic formation with one complete phosphorus dimer positioned in the center. Panel (b) depicts a single complete icosahedron with twelve external $\mathrm{B}$ and $\mathrm{P}$ atoms it is bound to. Boron atoms bound to an external boron atom (B-bound) are indicated by a light grey sphere, whereas boron atoms bound to an external phosphorus atom (P-bound) are indicated by black spheres. Boron-boron, boron-phosphorus and phosphorus-phosphorus bonds are drawn up to a maximum of 2,2 and $3 \AA$, respectively.

respectively, which compare well to the experimental values of $5.987 \mathrm{~nm}$ and $11.847 \mathrm{~nm}$ reported in literature[79]. As mentioned in the introduction, the structure is formed by boron icosahedra that form an orthorhombic lattice with phosphorus dimers in between. The crystal structure has only three symmetrically unique crystal lattice sites, a phosphorus site, a boron site externally bound to a boron atom from another icosahedron and a boron site bound to a phosphorus atom. These different boron sites will be referred to as P-bound and B-bound boron sites and are shown in Fig. 5.2 (b) as black and light gray spheres, respectively. Note that the B-bound boron sites are organized in two groups of three on opposing sides of the icosahedron and the P-bound boron atoms form a connected chain along the icosahedron, and these distinct groups of icosahedral atoms will be referred to as the poles and equator of the icosahedron, respectively. This asymetry will prove to play an important role in the diffusion characteristics of interstitial defects, which will be discussed later in this work. 


\subsubsection{INTERSTITIAL AND VACANCY DEFECTS}

To study the diffusion characteristics of interstitial and vacancy point defects, one first needs to determine their equilibrium positions within the crystalline host cell. The two unique boron lattice sites in the $\mathrm{B}_{12} \mathrm{P}_{2}$ crystal structure, give a total of 2 symmetrically unique boron vacancy defects, a B-bound boron vacancy $\mathrm{V}_{\mathrm{B}(\mathrm{B})}$ and a P-bound vacancy $\mathrm{V}_{\mathrm{B}(\mathrm{P})}$. In stark contrast with vacancy point defects, which occupy lattice sites of the crystal host by definition, the locations of interstitial point defects are not known $a$ priori. A reasonable initial guess, are positions within the crystal unit cell that are farthest removed from all occupied lattice sites, as positions that are too close to other atoms are unlikely to be thermodynamically stable. To determine these positions for $\mathrm{B}_{12} \mathrm{P}_{2}$, an interstitial boron atom was placed randomly within the unit cell, which was subsequently moved by a simplex algorithm to maximize its first-nearest neighbor distance. This process was repeated many times and a histogram of the optimized firstnearest neigbor distances, for all the randomly sampled starting interstitial positions, is shown in Fig. 4.3 (a). The positions within the crystalline unit cell, correspoding to the four most abundant first-nearest neighbor distances, labeled $A-D$ in Fig. 4.3 (a), are shown in Fig. 4.3 (b) and 4.3 (c).

Interstitial positions $A-C$ all have six symmetrically equivalent positions in the unit cell and interstitial position $D$ is located in the center of the icosahedron. A $2 \times 2 \times 2$ supercell was constructed with a single interstitial boron atom for each of the four different starting positions shown in Fig. 4.3, and the atomic positions were optimized by minimizing the Hellman-Feynman forces, acting on every individual atom, below $0.02 \mathrm{eV}^{-1}$. Parts of the relaxed structures, including the interstitial boron atom, are shown in Fig. 4.4.

The interstitials $A$ and $D$ relax to the same final structure, shown in Figs. 4.4 (a) and 4.4 (c), where the interstitial boron atom is fully incorporated in the icosahedron. The interstitial atom is located in the center of the triangle formed by the three B-bound boron atoms at the pole of the icosahedron, and is fully fivefold coordinated with icosahedral boron atoms, but does not form a bond with any extra-icosahedral atoms. To accommodate the interstitial, the B-bound atom indicated by the black sphere in Figs. $4.4(\mathrm{a})$ and $4.4(\mathrm{c})$, is significantly displaced to the point where it is no longer fivefold but fourfold coordinated with icosahedral atoms.

Interstitial $B$ and $C$ also relax to an identical configuration, as shown in Figs. 4.4 (b) and $4.4(\mathrm{~d})$. The interstitial boron atom is located next to an icosahedron, to which it is twofold coordinated. The directly adjacent phosphorus dimer maintains its orientation along the $\langle 001\rangle$ direction, but is significanly stretched to almost $3 \AA$ to allow both $\mathrm{P}$ atoms to coordinate with the interstitial. The interstitial and $\mathrm{P}$ dimer form an almost perfect isosceles triangle with the boron-phosphorus bonds, with length $1.87 \AA$, as the legs and a vertex angle $\angle \mathrm{PBP}$ of $104.8^{\circ}$. 

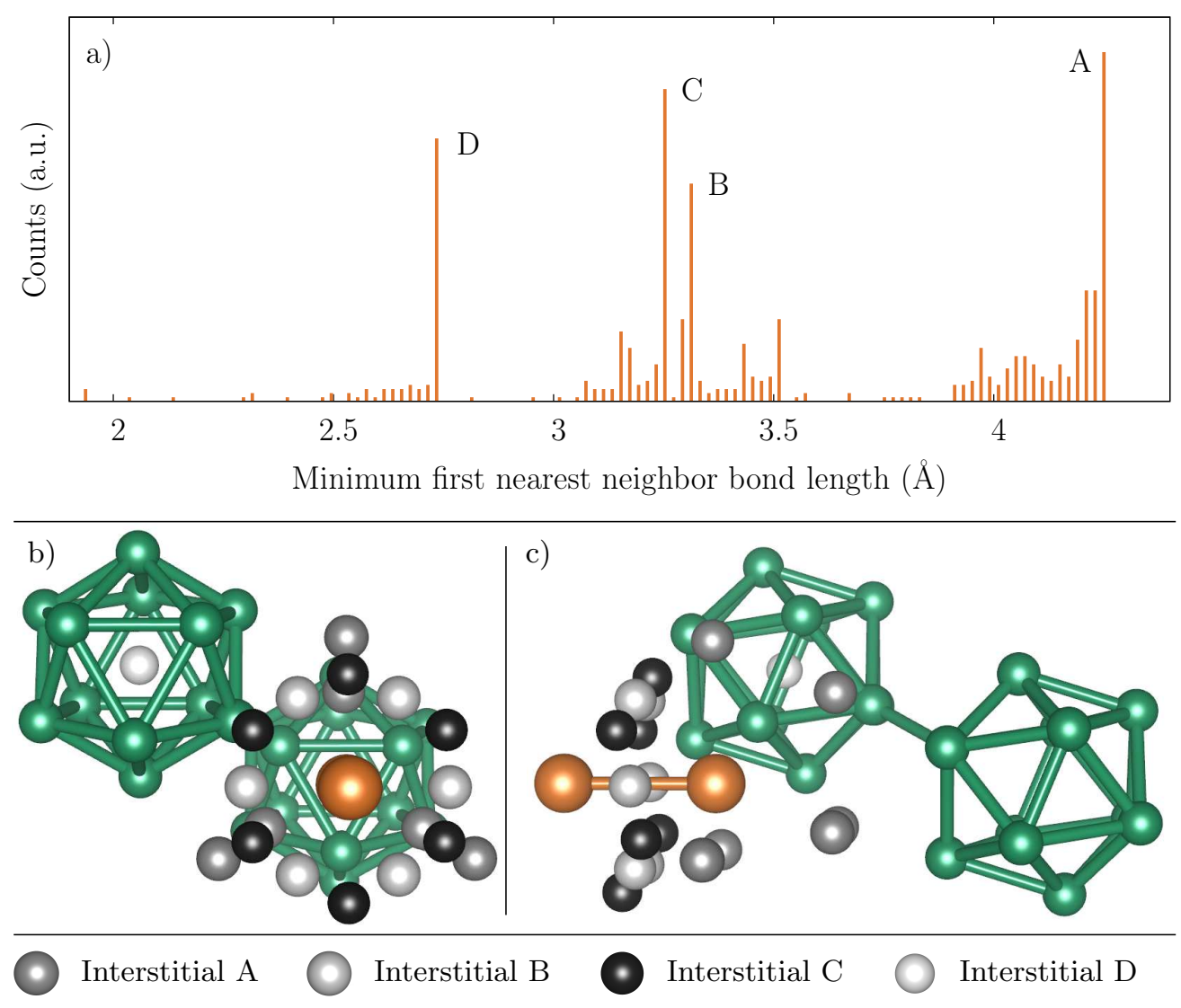

Figure 4.3: Histogram of optimized minimum nearest-neighbor distance of a boron interstitial for thousand randomly sampled starting positions. The most abundant optimized distances, labeled $A-D$, have the corresponding position within the unit cell shown (b) and (c). Site $A$ has 6 symmetrically identical positions within the unit cell. Sites $B$ and $C$ both have 6 symmetrically equivalent positions forming a buckled hexagon with the phosphorus dimer oriented along its center axis. Site $D$ is located at the center of the icosahedron. 

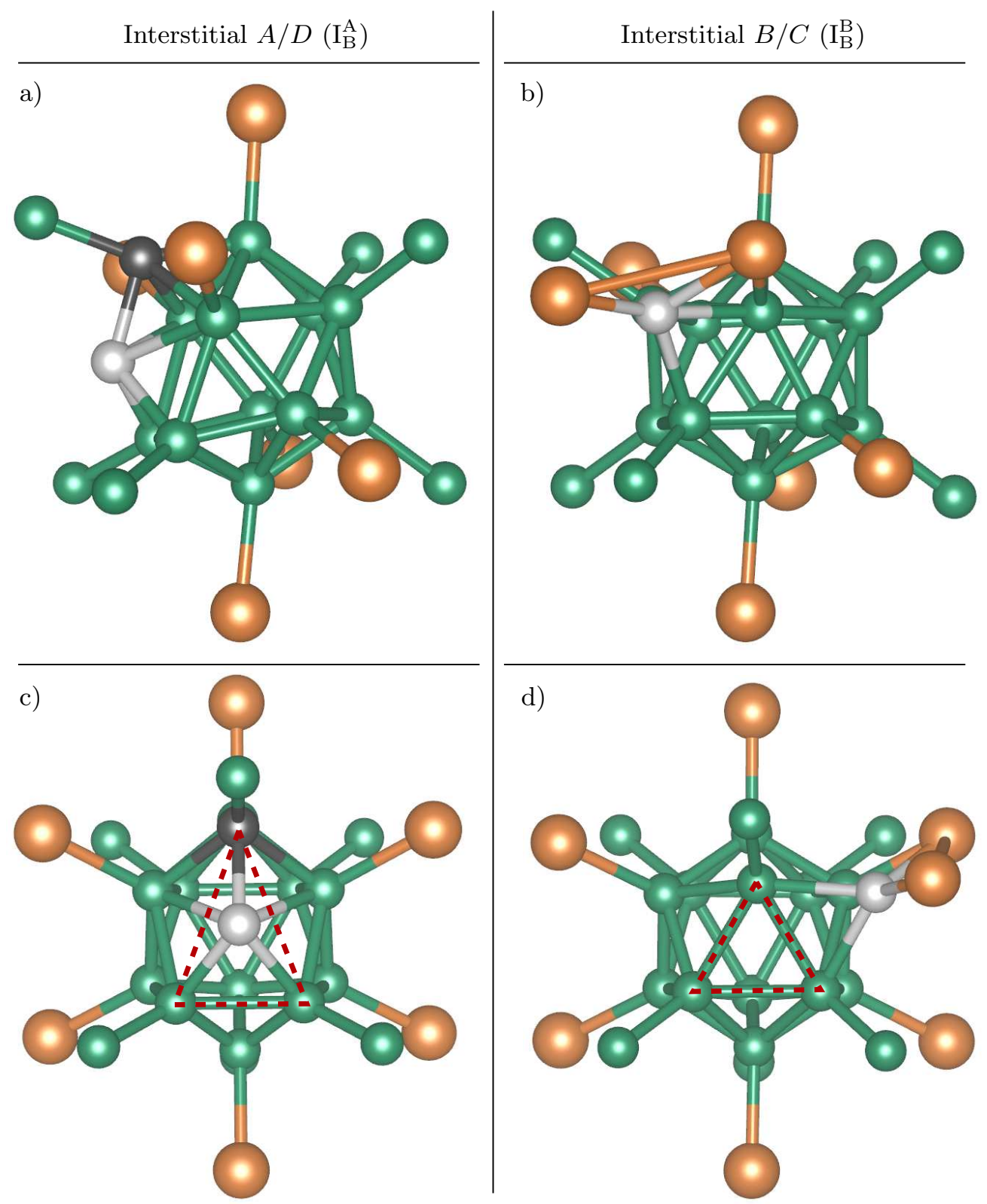

Figure 4.4: Side $(\mathrm{a}, \mathrm{b})$ and front $(\mathrm{c}, \mathrm{d})$ views of part of the relaxed supercells containing an interstitial boron atom. Interstitials $A / D$ and $B / C$ relax to the structures shown in (a,c) and (b,d), respectively, where the labels $A-D$ refer to the starting position of the interstitial atom before relaxation and correspond to the labels in Fig. 4.3. The light gray sphere indicates the interstitial atom and the black sphere in (a) and (c) indicates the undercoordinated B-bound atom. 
The two interstitial boron configurations as described in this section will be referred to as interstitial $A\left(\mathrm{I}_{\mathrm{B}}^{\mathrm{A}}\right)$ and interstitial $B\left(\mathrm{I}_{\mathrm{B}}^{\mathrm{B}}\right)$, respectively, throughout the rest of this work, where the subscript indicates the elemental type of the defect and the superscript is a label. We have created each one of the two interstial and vacancy defects, in a $2 \times 2 \times 2 \mathrm{~B}_{12} \mathrm{P}_{2}$ supercell and the formation energies of the relaxed defects $\mathrm{I}_{\mathrm{B}}^{\mathrm{A}}$ and $\mathrm{I}_{\mathrm{B}}^{\mathrm{B}}$, are $4.84 \mathrm{eV}$ and $5.06 \mathrm{eV}$, respectively. The vacancy defects $\mathrm{V}_{\mathrm{B}(\mathrm{B})}$ and $\mathrm{V}_{\mathrm{B}(\mathrm{P})}$, have very similar formation energies at $5.02 \mathrm{eV}$ and $5.45 \mathrm{eV}$, respectively. All of the defect structures relaxed with only minor deformations of the defective icosahedron or any of the directly neighboring icosahedra, which shows that a boron icoshedron maintains structural integrity even when in a degraded state in the presence of vacancy or interstitial defect.

\subsubsection{FRENKEL DEFECT RECOMBINATION PATHWAYS}

Now that we have obtained the equilibrium positions of vacancy and interstitial defects within the $\mathrm{B}_{12} \mathrm{P}_{2}$ unit cell, we can start to investigate potential recombination pathways of a vacancy and interstitial defect pair, often referred to as a Frenkel defect or Frenkel pair. In this work, we assume the existence of such a Frenkel defect and do not presuppose anything about the event that created the defect and as such our starting situation is a perfect $\mathrm{B}_{12} \mathrm{P}_{2}$ crystal with a single boron vacancy defect and a single boron interstitial, that are not necessarily located on the same icosahedron. Due to computational resource and time constraints, we limit the investigation to Frenkel defects with an interstitial defect of type $\mathrm{I}_{\mathrm{B}}^{\mathrm{A}}$. The formation energies indicate that this interstitial is thermodynamically more stable compared to $\mathrm{I}_{\mathrm{B}}^{\mathrm{B}}$, and therefore one can make the assumption that Frenkel recombination involving $\mathrm{I}_{\mathrm{B}}^{\mathrm{A}}$ interstitials, will serve as an upper limit for diffusion barrier energies of Frenkel defect recombination in general. Even when limiting the discussion to Frenkel defects consisting of an $\mathrm{I}_{\mathrm{B}}^{\mathrm{A}}$ interstitial and one of the two distinct boron vacancies, computing all combinations and potential recombination pathways would be computationally prohibitive. Instead, the full recombination process can be split into three subprocesses: (1) the diffusion of the vacancy, (2) the diffusion of the interstitial and finally (3) the diffusion of the interstitial to the vacancy and the recombination of the Frenkel pair.

\subsubsection{VACANCY DIFFUSION}

In the process of vacancy diffusion, we can distinguish between inter-icosahedral and intra-icosahedral vacancy diffusion, which correspond to migration of the vacancy site between two different icosahedra or within an icosahedron, respectively. For intraicosahedral diffusion of a boron vacancy to a directly neighboring lattice site, there are three distinct possibilities: $\mathrm{V}_{\mathrm{B}(\mathrm{B})}$ to $\mathrm{V}_{\mathrm{B}(\mathrm{B})}, \mathrm{V}_{\mathrm{B}(\mathrm{B})}$ to $\mathrm{V}_{\mathrm{B}(\mathrm{P})}$ and $\mathrm{V}_{\mathrm{B}(\mathrm{P})}$ to $\mathrm{V}_{\mathrm{B}(\mathrm{P})}$, where the notation $\mathrm{V}_{\mathrm{B}(\mathrm{P})}$ indicates a vacancy at a $\mathrm{P}$-bound boron site. The diffusion pathways 


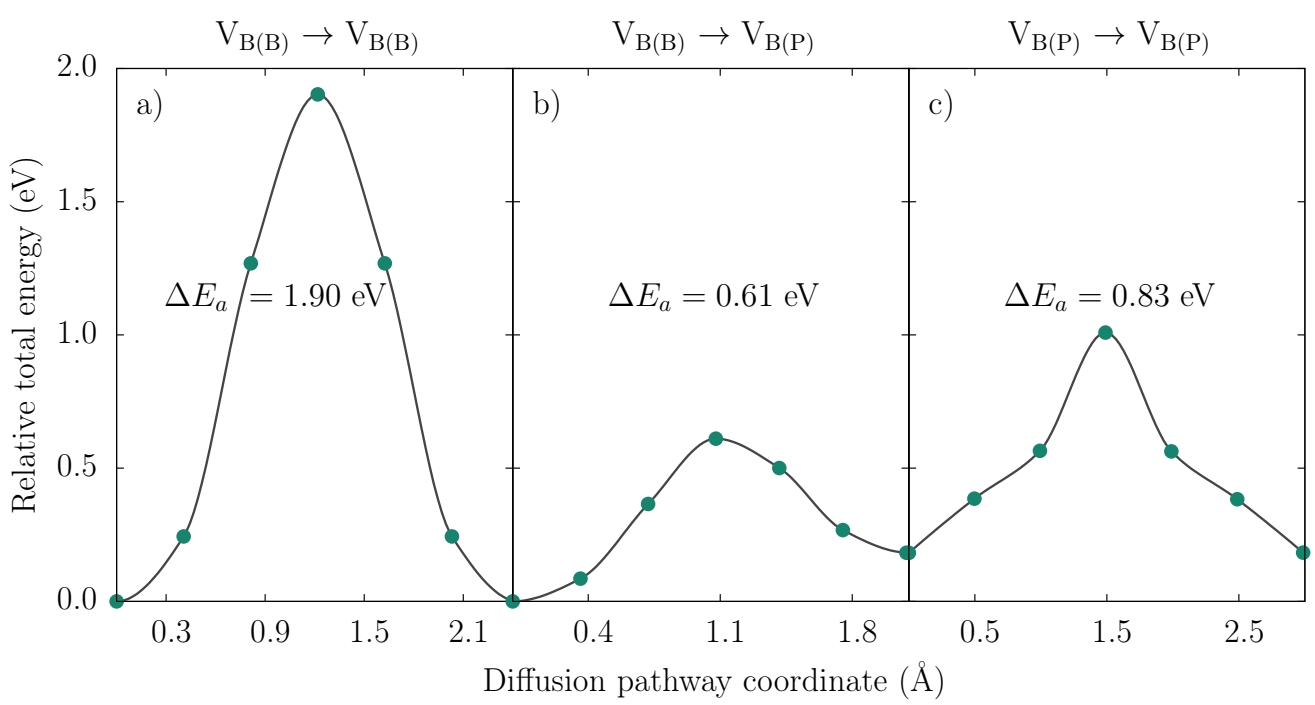

FiguRE 4.5: Diffusion pathways of a boron vacancy to a neighboring position on the same icosahedron from a (a) boron- to boron-bound site, (b) boron- to phosphorus-bound site and (c) a phosphorus- to phosphorus-bound site. The green points represent the total energy of the image along the diffusion pathway relative to the initial image and the solid line, computed by a piecewise cubic hermite interpolating polynomial, serves as a guide to the eye. The reaction coordinate of each image represents its total Cartesian distance relative to the initial image.

for each one of these three vacancy diffusion combinations have been computed using a nudged elastic band calculation, with 5 intermediate images along the pathway between the initial and final image, and the energy barrier profiles are shown in Fig. 4.5.

The diffusion activation energy $\Delta E_{a}$ is defined as

$$
\Delta E_{a}=E_{m}-E_{i}
$$

where $E_{i}$ is the total energy of the initial image and $E_{m}$ is the maximum energy along the diffusion pathway. Intra-icosahedral boron vacancy diffusion has the lowest activation energy when the initial and final vacancy site are boron sites bound to opposite elements, as can be seen in Fig. 4.5 (b), where diffusion of a boron- to phosphorusbound vacancy has a diffusion barrier of $0.61 \mathrm{eV}$, and $0.43 \mathrm{eV}$ in the opposite direction. The diffusion barrier for the $\mathrm{V}_{\mathrm{B}(\mathrm{P})}$ to $\mathrm{V}_{\mathrm{B}(\mathrm{P})}$ pathway is, at $0.83 \mathrm{eV}$, only slighly larger, but contrastingly, the $\mathrm{V}_{\mathrm{B}(\mathrm{B})}$ to $\mathrm{V}_{\mathrm{B}(\mathrm{B})}$ diffusion barrier is significantly larger at $1.90 \mathrm{eV}$. This strong asymmetry in diffusion activation energies is somewhat suprising, as at a first glance the defect structures along the different pathways look similar. Upon closer inspection, differences can be found however and certain structural parameters that capture the asymmetry are displayed in Table 4.1. 


\begin{tabular}{lcccc}
\hline Defect & $d_{\text {ico }}(\AA)$ & $d_{\text {ext }}(\AA)$ & $d_{\text {tot }}(\AA)$ & $l(\mathrm{~B}-\mathrm{B})(\AA)$ \\
\hline $\mathrm{V}_{\mathrm{B}(\mathrm{B})} \rightarrow \mathrm{V}_{\mathrm{B}(\mathrm{B})}$ & 0.63 & 0.56 & 1.20 & 1.67 \\
& & & & 1.68 \\
$\mathrm{~V}_{\mathrm{B}(\mathrm{B})} \rightarrow \mathrm{V}_{\mathrm{B}(\mathrm{P})}$ & 0.35 & 0.62 & 0.97 & 1.79 \\
$\mathrm{~V}_{\mathrm{B}(\mathrm{P})} \rightarrow \mathrm{V}_{\mathrm{B}(\mathrm{P})}$ & 0.52 & 0.54 & & 1.06 \\
\hline
\end{tabular}

TABLE 4.1: Structural parameters for the image along the diffusion pathway where the total relative energy is at a maximum for the three different types of vacancy diffusion. The second and third column show the summed distance in $\AA$ traveled by atoms, with respect to their position at the start of the diffusion pathway, from the defective icosahedron (excluding the diffusing atom itself) and from atoms that are a direct neighbor to the defective icosahedron, respectively, and the fourth column is the sum of these two distances. The final column shows the boron bond lengths in $\AA$ of the diffusing atom.

The columns labeled $d_{\text {ico }}$ and $d_{\text {ext }}$ represent the displacement of atoms in the image with the highest energy with respect to the initial image (i.e. the image in the middle of the diffusion pathway), for atoms of the icosahedron containing the vacancy and its directly neighboring external atoms, respectively. This value for the icosahedral atoms, is a direct measure of the distortion of the icosahedron under the influence of the diffusing vacancy and is a factor of two larger for the $V_{\mathrm{B}(\mathrm{B})} \rightarrow \mathrm{V}_{\mathrm{B}(\mathrm{B})}$ pathway compared to the $\mathrm{V}_{\mathrm{B}(\mathrm{B})} \rightarrow \mathrm{V}_{\mathrm{B}(\mathrm{P})}$ pathway. The reason for the increased icosahedral distortion can be traced back to the strongly contracted boron bond lengths of the diffusing boron atom (compared to the equilibrium boron-boron bond length of $1.79 \AA$ in the perfect crystal) as displayed in the last column of Table 4.1. In the image with the highest energy along the pathway, the diffusing boron atom has exactly two neighboring boron atoms with which it forms a bond and therefore each row in the last column of Table 4.1 contains two values, corresponding to the lengths of those boron bonds. The increased icosahedral distortion, due to contracted boron bond lengths of the diffusing boron atom, explains the significant energy barrier difference between the three different vacancy diffusion paths.

With a diffusion barrier of almost $2 \mathrm{eV}$, the $\mathrm{V}_{\mathrm{B}(\mathrm{B})} \rightarrow \mathrm{V}_{\mathrm{B}(\mathrm{B})}$ diffusion pathway is unlikely to occur, however the $\mathrm{V}_{\mathrm{B}(\mathrm{B})} \rightarrow \mathrm{V}_{\mathrm{B}(\mathrm{P})}$ pathway has an energy barrier that is certainly surmountable. Combined with the fact that a vacancy at any vertex on the icosahedron can reach all other vertices, solely by $\mathrm{V}_{\mathrm{B}(\mathrm{B})} \rightarrow \mathrm{V}_{\mathrm{B}(\mathrm{P})}$ diffusion, we find that a vacancy can diffuse intra-icosahedrally at a cost of approximately $0.55 \mathrm{eV}$ per step.

For inter-icosahedral vacancy diffusion, we consider the same three variations as discussed for inter-icosahedral diffusion in the previous section, and we will limit the discussion to lattice position pairs with the shortest possible distance. The results are shown in Fig. 4.6. 


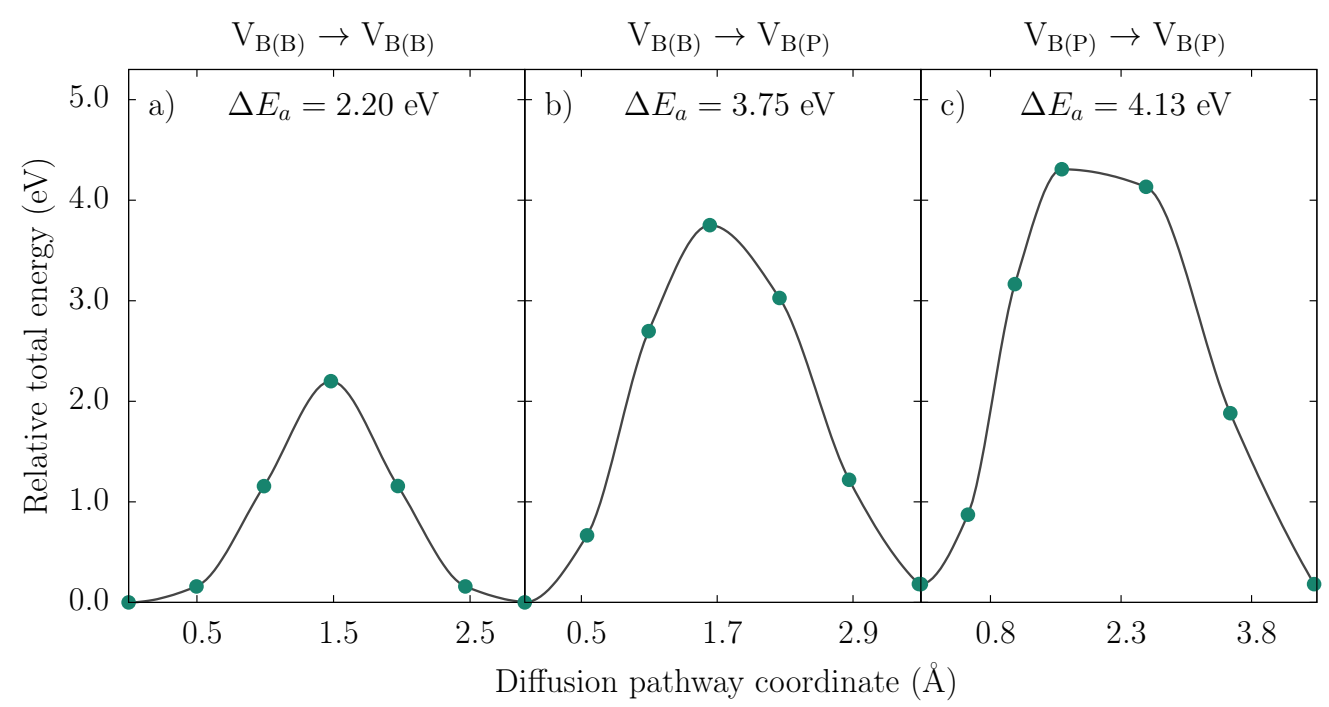

FIGURE 4.6: Diffusion pathways of a vacancy to a position on a neighboring icosahedron from a (a) boron- to boron-bound site, (b) boron- to phosphorus-bound site and (c) a phosphorusto phosphorus-bound site. The green points represent the total energy of the image along the diffusion pathway relative to the initial image and the solid line, computed by a piecewise cubic hermite interpolating polynomial, serves as a guide to the eye. The reaction coordinate of each image represents its total Cartesian distance relative to the initial image.

The activation barriers for all considered inter-icosahedral vacancy diffusion pathways are significantly larger, compared to their corresponding intra-icosahedral analogs. At multiple $\mathrm{eV}$ at least, these diffusion events are highly unlikely to occur spontaneously or due to thermally activated processes. Even for the shortest diffusion pathway $V_{B(B)}$ $\rightarrow \mathrm{V}_{\mathrm{B}(\mathrm{B})}$, which models the diffusion of a polar boron atom to the closest polar position on its directly neighboring icosahedron, an activation barrier of $2.2 \mathrm{eV}$ ensures that the probability of this diffusion process occuring is negligible.

\subsubsection{INTERSTITIAL DIFFUSION}

In this section, we will discuss interstitial diffusion, which we will limit to intraicosahedral interstitial diffusion, since we have seen from inter-icosahedral vacancy diffusion, that the migration of boron atoms between icosahedra is costly and unlikley to occur. In section 4.3.2, we described the interstitial boron positions in the $\mathrm{B}_{12} \mathrm{P}_{2}$ unit cell. When discussing the diffusion of interstitial defects, in the context of a Frenkel defect and its recombination, we only consider the most thermodynamically stable interstitial $\mathrm{I}_{\mathrm{B}}^{\mathrm{A}}$. In this interstitial configuration, one could consider the interstitial atom to be either the added atom without any extra-icosahedral bonds, or the displaced atom with a fourfold intra-icosahedral undercoordination, shown as light and 


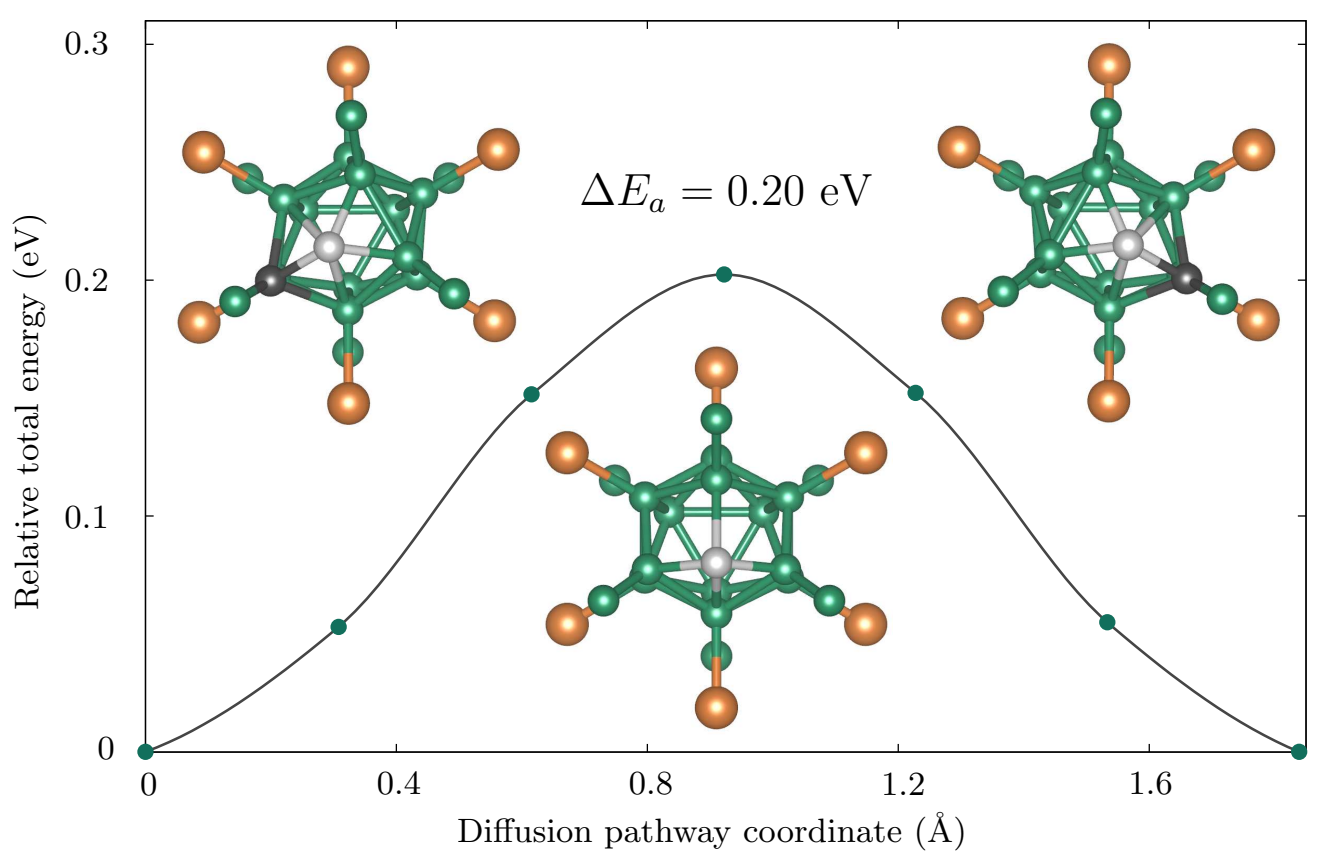

Figure 4.7: Diffusion barrier for polar interstitial diffusion with the total energy of each image, relative to the initial image, plotted as a function of the coordinate of the image along the diffusion pathway. The solid line serves as a guide to the eye. The structural configurations from left to right correspond to the initial, middle and final image along the diffusion pathway, respectively.

dark gray spheres in Fig. 4.4 (a) and (c), respectively. A more useful interpretation is to consider both these atoms as an interstitial pair and to study their combined intraicosahedral diffusion behavior. We recall the pattern found in the elemental types of directly neighboring atoms of the boron icoshedron [see Fig. 5.2 (b)], where the B-bound atoms are grouped into two triangles at the poles of the icosahedron and the P-bound boron atoms form a connected ring along the equator. The interstitial pair is formed by one of the atoms of the polar triangle and an atom in the center of the triangle, and symmetry dictates that there are six symmetrically equivalent configurations of this interstitial pair.

Interstitial diffusion can then be defined as the process where the interstitial pair transitions between any of these six configurations, which again due to symmetry can be reduced to two distinct diffusion pathways: (1) diffusion within the same polar triangle (polar diffusion) or (2) diffusion from one pole, across the icosahedral equator to the opposing pole (cross-equatorial diffusion). The diffusion pathway for polar interstitial diffusion as calculated with the NEB method, is shown in Fig. 4.7. The structural configurations of the initial, middle and final image along the diffusion pathway show 


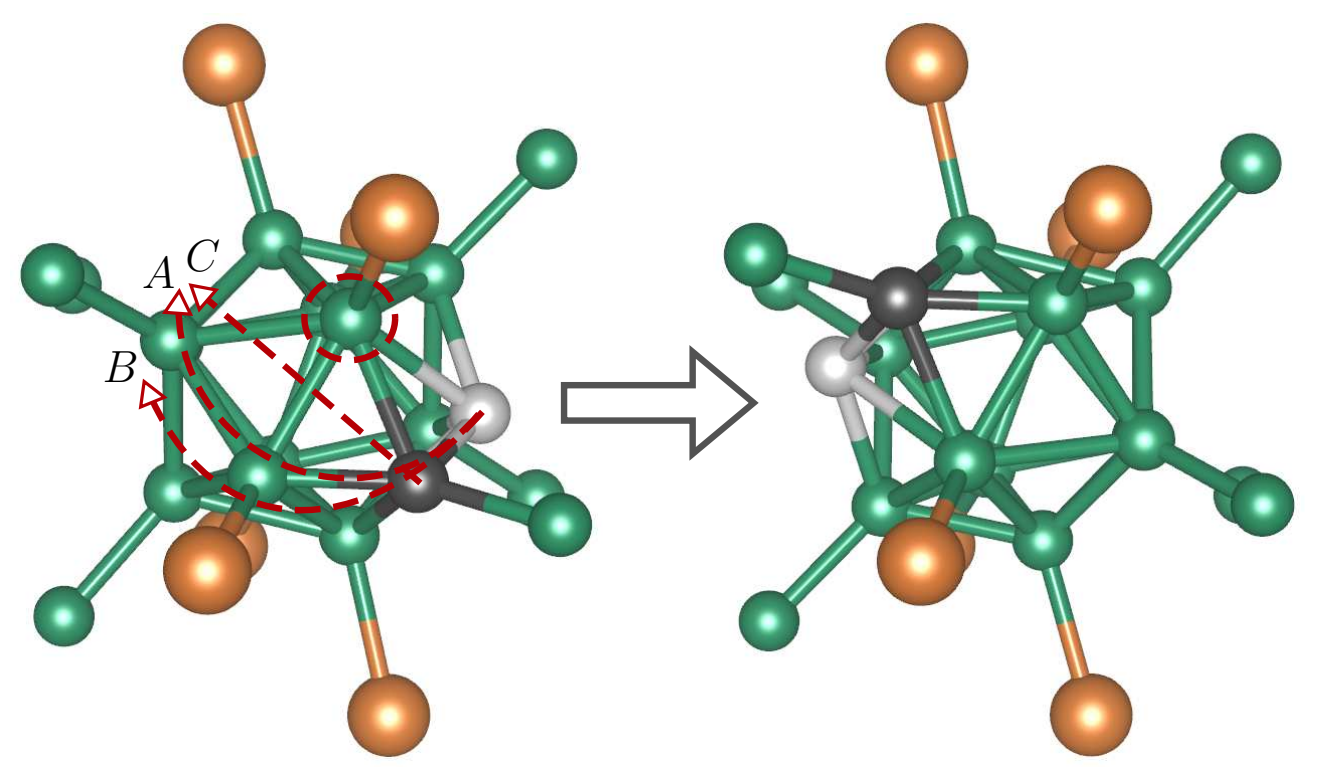

FIGURE 4.8: From left to right, the initial and final image for cross-equatorial interstitial diffusion. The atoms belonging to the interstitial pair are represented by light and dark gray spheres as in all previous figures. The red arrows labeled, $A, B$ and $C$ represent a schematic pathway along which the atoms diffuse. For pathways $A$ and $B$ both atoms of the interstitial pair move along the direction of the arrow, pushing neighboring boron atoms along the direction of the arrow, across the icosahedron equator. The arrow labeled $C$ indicates a diffusion pathway where only the interstitial atom, marked by the dark gray sphere, diffuses directly across the equator.

that the relative ordering of the atoms is maintained and the distortion of the polar triangle, due to the presence of the interstitial atom, is merely transfered to the other vertex of the triangle by a skewing motion. As such, the required atomic displacement for the diffusion of the interstitial pair is minimal and the diffusion activation energy, at $0.20 \mathrm{eV}$, is relatively low compated to vacancy diffusion. Through this skewing motion, the interstitial pair can thus reach any of the two other configurations on the same pole, and with that, reach half the space bordering the icosahedron, at a minimal energy cost.

In the case of cross-equatorial interstitial diffusion, the interstitial pair has to diffuse from one pole of the icosahedron to the other, crossing the icosahedron's equator. The initial and final image of such an interstitial diffusion pathway are shown in Fig. 4.8.

A migration of the interstitial atom directly through the center of the icosahedron will have too large an energy barrier and will be a highly unlikely event. Alternatively, the interstitial pair can migrate over the surface of the icosahedron, effectively by moving over to the directly adjacent occupied icosahedral vertex, pushing its current 


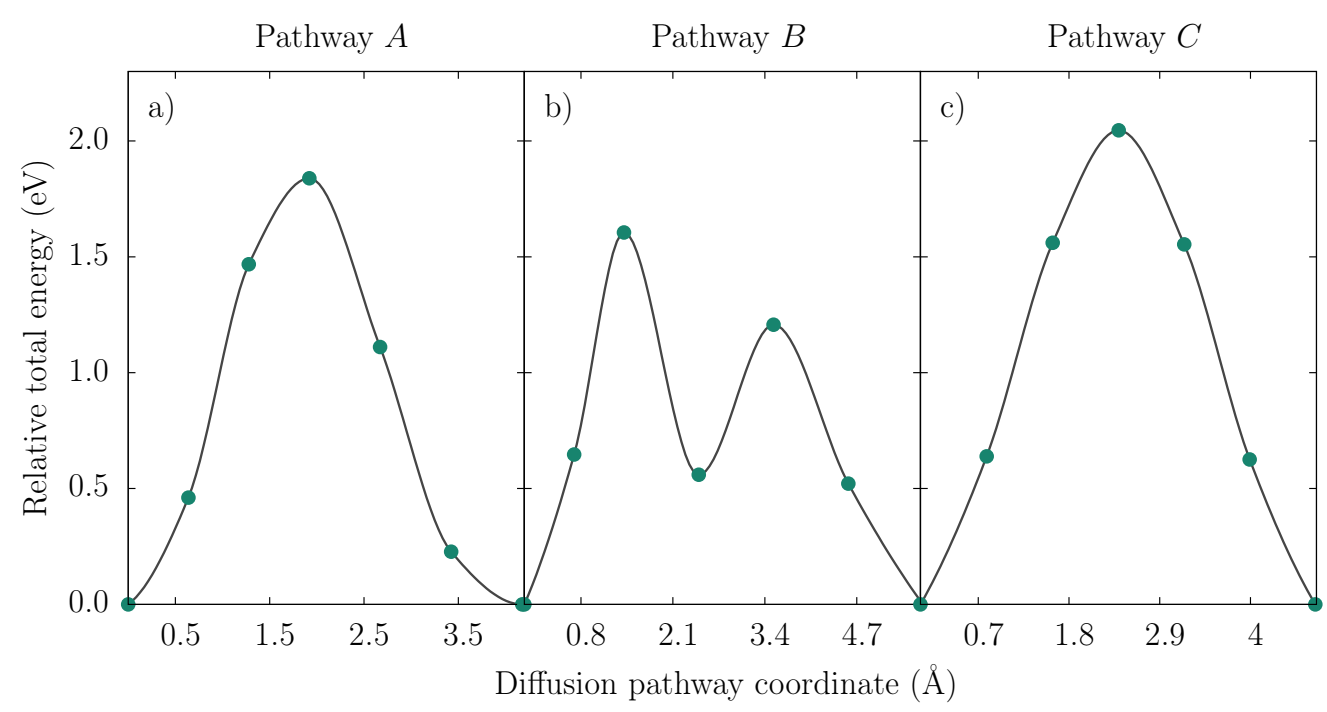

Figure 4.9: Equatorial interstitial diffusion barriers for pathways (a) $A$, (b) $B$ and (c) $C$ from Fig. 4.8. The green points indicate the total energy of the image relative to the initial image as a function of the pathway coordinate of the image. The solid curve serves as a guide to the eye.

occupant towards the other side of the equator. Two of such pathways, labeled $A$ and $B$, have been schematically indicated by red arrows in Fig. 4.8, where the diffusing atoms will move roughly along the direction of the arrow. It is important to note that the atoms of the interstitial pair in the initial image, indicated by the light and dark gray spheres, do not actually move to the other pole themselves but rather they move along the direction of the arrow, pivoting around the P-bound atom highlighted by a red circle, pushing atoms along their way across the equator. Contrastingly, the arrow labeled $C$, represents a diffusion pathway where the dark gray atom directly crosses the equator. The diffusion barriers for the three different pathways $A, B$ and $C$ are shown in Fig. $4.9(\mathrm{a}-\mathrm{c})$, respectively.

Compared to the polar interstitial diffusion, the energy barriers for cross-equatorial interstitial diffusion are significantly higher at $1.6 \mathrm{eV}$ to $2.1 \mathrm{eV}$. This is understandable as for cross-equatorial diffusion the total atomic displacement along the pathway is significantly larger and during diffusion bonds are broken. The structure of the system that causes the local minimum in the middle of the diffusion path of pathway $B$ [see Fig. 4.9 (b)], corresponds directly to the configuration of interstitial $\mathrm{I}_{\mathrm{B}}^{\mathrm{B}}$, as shown in Fig. 4.4. These three diffusion pathways have the smallest diffusion energy barrier possible for cross-equatorial interstitial diffusion and therefore it is likely that, once an interstitial of type $\mathrm{I}_{\mathrm{B}}^{\mathrm{A}}$ is formed on a certain pole of an icosahedron, it is most likely trapped on that side of the equator. 
In summary, we find that cross-equatorial diffusion of an interstitial pair is unlikely due to a high diffusion activation energy, which is in stark contrast with polar diffusion which, with a diffusion barrier of a mere $0.20 \mathrm{eV}$ is more likely to occur. Despite the icosahedral equator formed by P-bound boron atoms acting like a barrier for interstitial diffusion, preventing an interstitial pair from easily diffusing to the opposing pole, it can still reach half of the space directly adjacent to the icosahderon through polar interstitial diffusion.

\subsubsection{FRENKEL DEFECT RECOMBINATION}

With the analysis of the diffusion behavior of vacancy and interstitial defects completed, we can now turn to the final part of Frenkel defect diffusion; the recombination of the interstitial with the vacancy. The most likely diffusion paths of the interstitial atom will be those where the vacancy lies on the face of an icosahedron directly facing the pole of the interstitial pair. For any other paths, the interstitial will have to diffuse around or through icosahedra or phosphorus dimers, and would therefore be energetically costly and unlikely to occur.

We consider an interstitial defect of type $\mathrm{I}_{\mathrm{B}}^{\mathrm{A}}$, which has three directly neighboring icosahedra, two of which have a symmetrically identical orientation with respect to the boron interstitial pair. There are three boron atoms on the interstitial pole that have a symmetrically unique orientation with respect to neighboring icosahedra, indicated by dark gray spheres in Fig. 4.10, and labeled $I_{a}, I_{b}$ and $I_{c}$. Potential vacancy defect sites are sites on the icosahedral faces that are directly adjacent to the interstitial defect, marked by light gray spheres in Fig. 4.10 and labeled $V_{a}$ through $V_{l}$. The labels $V_{a}$ through $V_{l}$ and $I_{a}$ through $I_{c}$ merely serve as indices to indentify their corresponding crystal lattice sites. Recombination pathways are considered for Frenkel defects where the interstitials $I_{a}, I_{b}$ and $I_{c}$ are paired up with a vacancy from the groups $V_{a}-V_{f}$, $V_{a}-V_{l}$ and $V_{g}-V_{l}$, respectively, as indicated by the arrows in Fig. 4.10, giving a total of 24 Frenkel pairs.

For every Frenkel pair combination, the initial and final image of its corresponding recombination pathway, are the cell containing a Frenkel defect and the perfect crystal, respectively. The initial recombination pathway was constructed with six linearly interpolated images between the fully relaxed initial and final image, giving a total of 8 images labeled 0 through 7 , where 0 corresponds to the initial image. A requirement of the NEB method, is that both the initial and final image, from which the intermediate pathway is constructed, are fully relaxed. In this case, relaxing the final image is trivial, since that always constitutes the perfect crystal where the Frenkel defect has fully recombined. Contrastingly, there is no guarantee that the initial image containing the Frenkel defect has a (meta)-stable state, which as it turns out is the case for 4 out of the 24 Frenkel defect configurations that we considered. For Frenkel defects with a vacancy at either $V_{d}$ or $V_{j}$, no stable state exists and the defect always spontaneously 
recombined and therefore a meaningful recombination pathway could not be computed and the activation barrier for the recombination of these Frenkel defects is effectively zero. This is not wholly surprising, considering that the boron atoms at vacancy positions $V_{d}$ and $V_{j}$ are the interconnecting atoms to the interstitial containing icosahedron, and are the closest to that icosahedron out of all the considered vacancy positions.

Interestingly, all other Frenkel defects do have a (meta)-stable state and the initial image could be fully relaxed without spontaneous recombination, and an attempt to find a relaxed diffusion pathway through a NEB calculation could be made. This shows that boron icosahedra are remarkably robust and remain stable, maintaining their structural integrity, even in the presence of a boron vacancy or interstitial defect and that due to the stability of these Frenkel defects, the diffusion pathway now has an activation energy that needs to be overcome, for recombination to occur. With the exception of four Frenkel defect pairs, $I_{b} \rightarrow V_{a}, I_{b} \rightarrow V_{b}, I_{b} \rightarrow V_{k}$ and $I_{b} \rightarrow V_{l}$, a relaxed diffusion recombination pathway could be found, from which an activation energy barrier could be extracted, as shown in Table 4.2. The reason that no relaxed solution could be found for the four aforementioned Frenkel recombination pathways is directly related to the fact that the corresponding vacancy positions are the furthest removed from the interstitial atom [see Fig. 4.10]. Moreover, the direct diffusion pathway from the interstitial to these vacancy positions is blocked by the boron-boron bond that interconnects the two involved icosahedra, which act as a barrier to the interstitial atom.

When constructing an initial guess for the recombination pathway of a Frenkel pair, the most straightforward assumption is that the interstitial atom diffuses directly to the position of the vacancy. However, this pathway is not necessarily the only possible pathway nor the most energetically favorable. This is best illustrated by an example, so we consider the recombination of Frenkel pair $I_{c} \rightarrow V_{g}$. The initial guess of the directly diffusing pathway will have interstitial $I_{c}$ diffuse directly to the vacancy at position $V_{g}$. However, one could also imagine a pathway where the boron atom at $V_{j}$ will diffuse to the vacancy at $V_{g}$, after which interstitial $I_{c}$ will diffuse to the newly created vacancy at $V_{j}$. The final images of these two scenarios are completely indistinguishable, however their recombination pathways, with activation energies of $1.41 \mathrm{eV}$ and $0.39 \mathrm{eV}$, respectively, are distinctively unique. These two types of pathways, where either the interstitial atom diffuses directly, or the recombinatorial diffusion is mediated by one or more other boron atoms, will be referred to as "direct" and "mediated" diffusive recombination, respectively.

The column labeled "Mediated" in Table 4.2 indicates whether the given activation energy corresponds to a mediated or direct diffusion recombination pathway. For some Frenkel pairs, both a direct and a mediated pathway could be found, in which case the one with the lowest activation energy is listed. In general, when pathways of both types were found, the mediated pathway had a lower activation energy, with the noticeable exception of $I_{c} \rightarrow V_{k}$ and $I_{c} \rightarrow V_{l}$, for which the mediated activation energy 


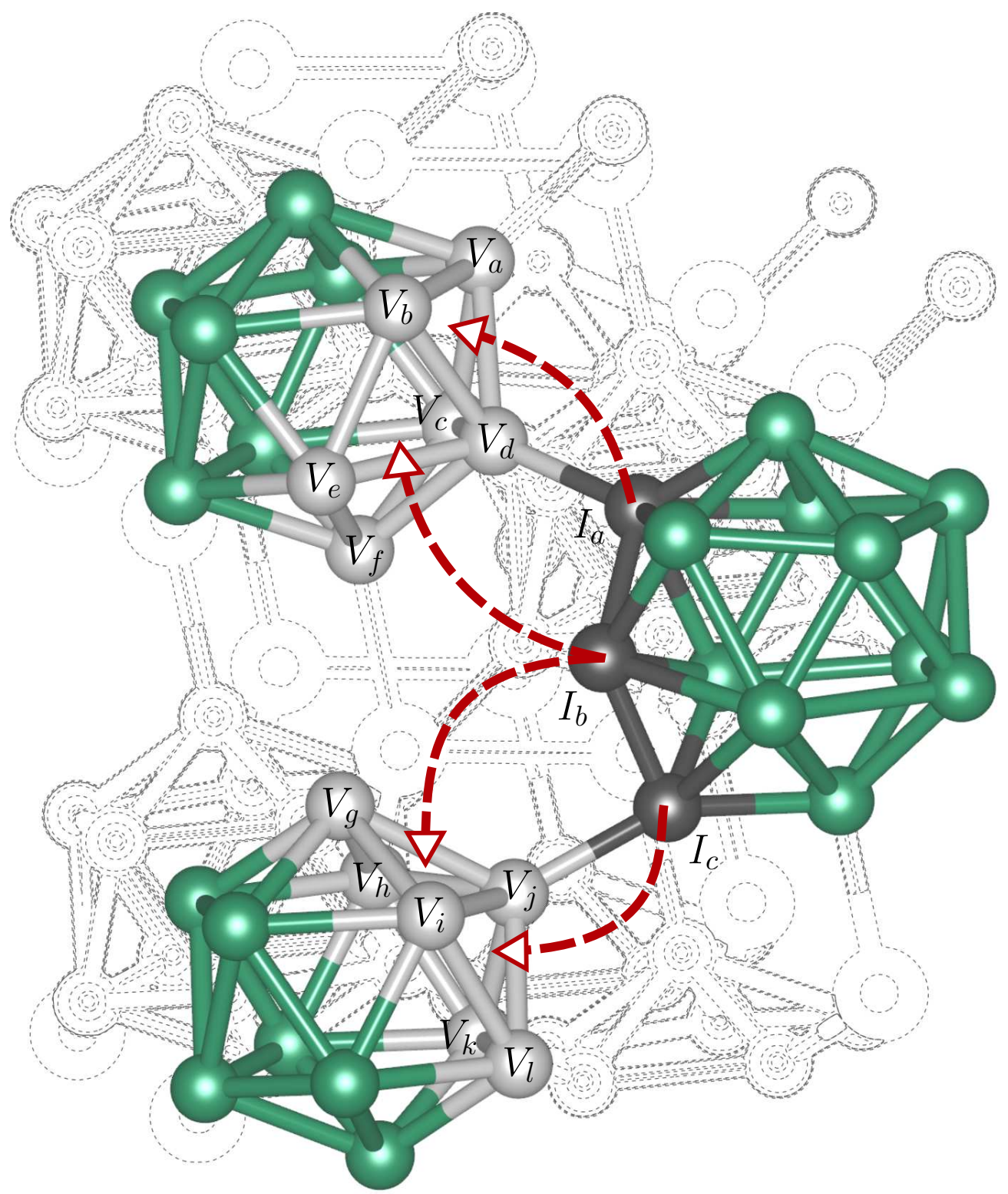

FIGURE 4.10: Atomic positions considered for the interstitial and vacancy defects of Frenkel pairs, indicated by dark gray and light gray spheres and labeled $I_{a}-I_{c}$ and $V_{a}-V_{l}$, respectively. The red arrows indicate the pairing up of the interstitials $I_{a}, I_{b}$ and $I_{c}$ with vacancies from the groups $V_{a}-V_{f}, V_{a}-V_{l}$ and $V_{g}-V_{l}$, respectively. 


\begin{tabular}{lccccc}
\hline Pair & $\Delta E_{a}$ & Mediated & Pair & $\Delta E_{a}$ & Mediated \\
\hline$I_{a} \rightarrow V_{a}$ & 0.04 & Yes & $I_{b} \rightarrow V_{a}$ & - & - \\
$I_{a} \rightarrow V_{b}$ & 0.003 & Yes & $I_{b} \rightarrow V_{b}$ & - & - \\
$I_{a} \rightarrow V_{c}$ & 0.24 & Yes & $I_{b} \rightarrow V_{c}$ & 0.25 & Yes \\
$I_{a} \rightarrow V_{d}$ & $\times$ & $\times$ & $I_{b} \rightarrow V_{d}$ & $\times$ & $\times$ \\
$I_{a} \rightarrow V_{e}$ & 0.31 & Yes & $I_{b} \rightarrow V_{e}$ & 0.31 & Yes \\
$I_{a} \rightarrow V_{f}$ & 0.38 & Yes & $I_{b} \rightarrow V_{f}$ & 0.40 & No \\
$I_{c} \rightarrow V_{g}$ & 0.39 & Yes & $I_{b} \rightarrow V_{g}$ & 0.02 & No \\
$I_{c} \rightarrow V_{h}$ & 0.16 & Yes & $I_{b} \rightarrow V_{h}$ & 0.31 & No \\
$I_{c} \rightarrow V_{i}$ & 0.16 & Yes & $I_{b} \rightarrow V_{i}$ & 0.31 & No \\
$I_{c} \rightarrow V_{j}$ & $\times$ & $\times$ & $I_{b} \rightarrow V_{j}$ & $\times$ & $\times$ \\
$I_{c} \rightarrow V_{k}$ & 0.23 & No & $I_{b} \rightarrow V_{k}$ & - & - \\
$I_{c} \rightarrow V_{l}$ & 0.23 & No & $I_{b} \rightarrow V_{l}$ & - & - \\
\hline
\end{tabular}

TABLE 4.2: Diffusion activation energies $\Delta E_{a}$ of recombination pathways of various Frenkel pairs in $\mathrm{eV}$. The interstitial and vacancy labels of the Frenkel pairs directly correspond to the labels in Fig. 4.10. Frenkel pairs without a stable state and therefore without a meaningful activation energy for their recombination are marked by $\times$. Frenkel pairs with a (meta)-stable state but for which no relaxed recombination pathway was found through the NEB algorithm are indicated by a dash.

is significantly larger at $0.68 \mathrm{eV}$, compared to the $0.23 \mathrm{eV}$ of the direct recombination pathway.

A schematic represenation of a mediated recombination pathway is shown in Fig. 4.11, which depicts four images of the $I_{a} \rightarrow V_{b}$ recombination pathway. The leftmost panel in Fig. 4.11, represents the initial image which contains a Frenkel defect, with the vacancy at position $V_{b}$ and the interstitial atom $I_{a}$, whereas the rightmost panel shows the final image where the Frenkel defect is recombined and the structure is a perfect crystal once again. The structures in Figs. 4.11 (b) and 4.11 (c) represent images along the pathway between the initial and final image. Interstitial atom $I_{a}$ is shown in black and the boron atom at position $V_{d}$ is indicated as a light gray sphere, which highlights that the diffusion of interstitial $I_{a}$ to the vacancy containing icosahedron, is in fact mediated by the diffusion of the atom at $V_{d}$ to the vacancy at $V_{b}$. Effectively then, this Frenkel recombination process is a summation of two separate diffusive processes, an intra-icosahedral vacancy- and an inter-icosahedral interstitial diffusion, that operate synchronously. The seamless coordination of these two diffusive processes in the case of $I_{a} \rightarrow V_{b}$, culminates in a negligible activation energy barrier for the recombination of the Frenkel defect.

The energy profile of a recombination pathway can be defined as the total energy of every image along the relaxed recombination pathway, relative to the initial image, as a function of the total cartesian distance of the image with respect to the initial image. 

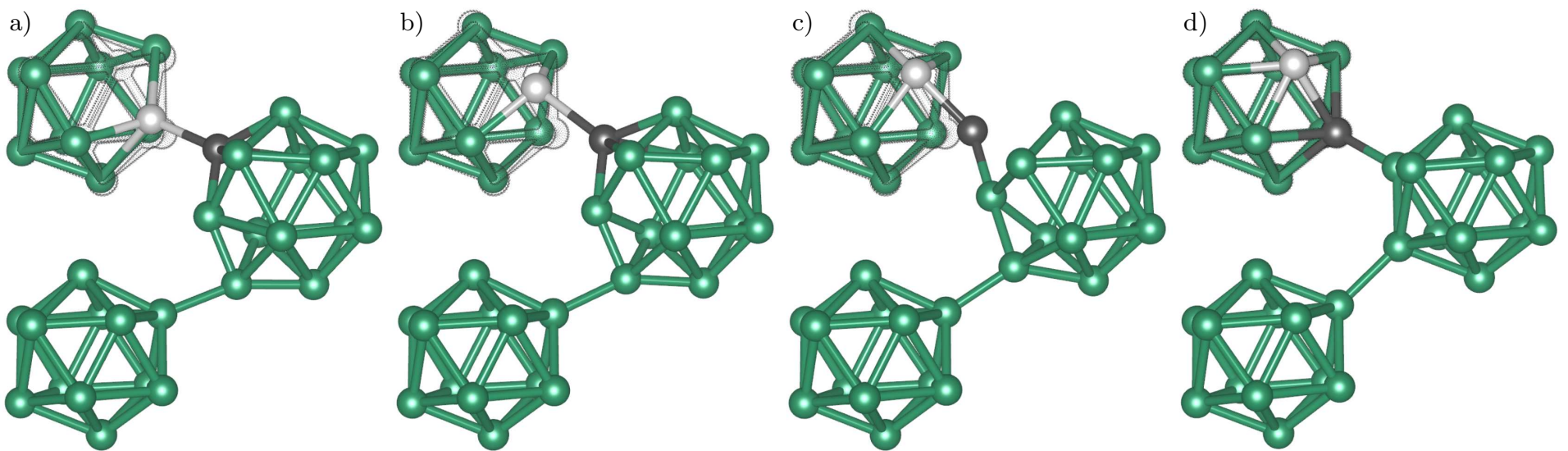

Figure 4.11: (a-d) Partial structures of the initial, second, fourth and final image, respectively of the relaxed recombination pathway for the $I_{a} \rightarrow V_{b}$ Frenkel defect. The interstitial atom $I_{a}$ is colored black and the mediating atom at position $V_{d}$ is colored in light gray. All four images show a black mesh outline over the top left vacancy containing icosahedron, which traces the atoms of the icosahedron in its relaxed configuration in the final image. This serves as a guide to the eye, to indicate the relaxed atomic positions that the diffusing atoms will end up in. 


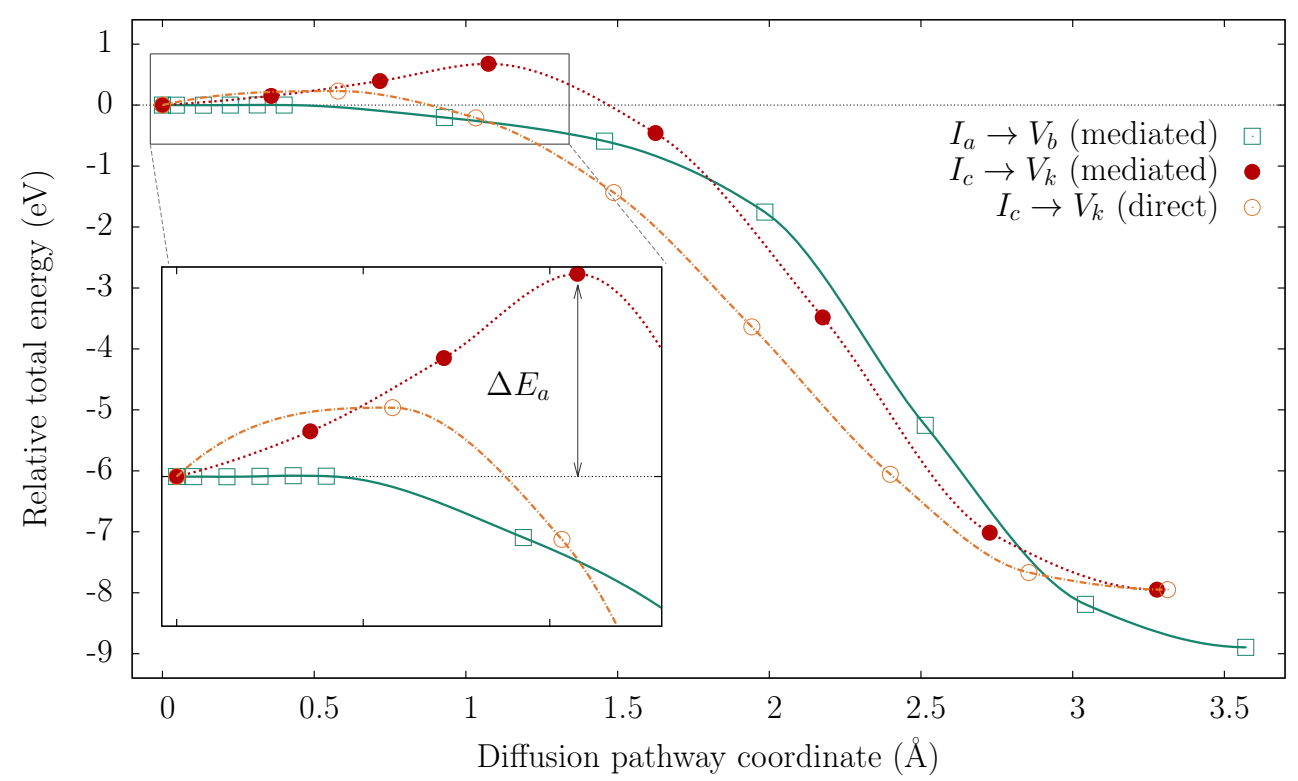

Figure 4.12: Energy profiles for three Frenkel recombination pathways. The symbols represent the total energies of the images along the pathway in $\mathrm{eV}$, relative to the initial image, as a function of the image's total Cartesian distance in $\AA$, with respect to the initial image. The solid line serves as a guide to the eye and the inset shows a close up of the region where the total energy of the pathways reaches a maximum.

Three examples, including the energy profile for the $I_{a} \rightarrow V_{b}$ recombination pathway that has just been discussed, are shown in Fig. 4.12.

The activation energy, indicated by $\Delta E_{a}$ in Fig. 4.12, is clearly visible for both the $I_{c} \rightarrow V_{k}$ pathways, but is almost indiscernible for $I_{a} \rightarrow V_{b}$. As mentioned previously, for the majority of Frenkel defects, the mediated recombination pathway often had a smaller activation energy compared to the direct recombination pathway. The pathways for $I_{c} \rightarrow V_{k}$ (and identically for $I_{c} \rightarrow V_{l}$ which is not shown) are the exception, where the activation energy is significantly larger for the mediated recombination pathway. In general, all the relaxed recombination pathways for the Frenkel pairs considered, have an energy profile with a similar shape. An initial monotonic incline in energy, which represents the activation energy barrier for the recombination, followed by a steep monotonic decline in energy, reaching a minimum for the final image. However, for the Frenkel defects $I_{a} \rightarrow V_{b}$ and $I_{b} \rightarrow V_{g}$, the entire energy profile of the recombination pathway was descending. Therefore a value for the activation energy could not be extracted, even though it is certain that the pathway has a non-negligble barrier, given that the defect was initially stable. For these Frenkel defects, the relaxed recombination pathway was used as a starting point for a second iteration, where additional images were added between the first and second image and the NEB method was used to relax 
this new pathway. The inset in Fig. 4.12 shows the additional images for the $I_{a} \rightarrow V_{b}$ Frenkel defect, from which the actual recombination activation energy of $3 \mathrm{meV}$ was determined.

The total energy of the final image is equal for all recombination pathways, as it is the total energy of the perfect crystal. The fact that the relative energies for the final images of the pathways, plotted in Fig. 4.12, are not equal, is due to the fact that these are relative to the total energy of the initial image of their respective Frenkel defect, which varies slightly between the different Frenkel pair configurations, but is typically approximately $8.9 \mathrm{eV}$ higher compared to the perfect crystal. This energy difference corresponds directly to the formation energy of the Frenkel pair and this is also the energy that will be released into the system upon the recombination of the Frenkel defect. The effect on the crystal structure of the sudden release of such an amount of energy, will certainly not be negligible and will be interesting for further study, however the simulation of these dynamics are beyond the scope of this work. The study of the dynamics of a system with a Frenkel defect prior to its recombination, however, is possible and of interest. Moreover, entropic effects due to the vibrational modes of the system are not properly described in the NEB method, however, these are explicitly accounted for in a molecular dynamics approach, provided that the system is sampled over sufficiently long time scales. The molecular dynamics approach therefore, though computationally expensive, provides a robust and valuable method of verifying the predicted free energy landscape by means of the NEB method.

\subsubsection{FRENKEL RECOMBINATION DYNAMICS}

For the 24 Frenkel defect configurations discussed in the previous section, the nudged elastic band method determined that 4 Frenkel defects do not have a stable initial state, for 4 Frenkel defects no relaxed combination pathway could be found and for the remaining 16 defects, a recombination activation energy between $0.003 \mathrm{eV}$ and $0.4 \mathrm{eV}$ was found. A drawback of the NEB method is that the final relaxed pathway, if one can be found, is dependent on the initial guess for the pathway. In this work, the initial guess was a simple linear interpolation between the initial and final image, with optional atomic index swapping, but other than that the pathway was restricted to that initial guess within certain margins, through the constraints of the NEB method. Consequently, if the initial guess is too far off from what would be the true optimal recombination pathway, the wrong pathway may be found or no path may be found at all where one does exist. To verify the conclusions made from the NEB analysis, we studied the molecular dynamics of Frenkel defects prior to their recombination, which does not suffer from the bias introduced by the initial guess of the recombination process, which is inherent in the NEB method.

The starting configuration for all Frenkel defect recombination dynamics simulations, is a $\mathrm{B}_{12} \mathrm{P}_{2}$ supercell with a single $\mathrm{I}_{\mathrm{B}}^{\mathrm{A}}$ defect, equilibrated at the target temperature $T$ 
as explained in detail in Section 4.2.3. After equilibration, whose point in time is set to be the origin $t=0$, in intervals of $1 \mathrm{ps}$, for each of the 12 potential locations labeled $V_{a}-V_{l}$ in Fig. 4.10, a single vacancy was created and the micro-canonical ensemble was sampled for a further 1 ps. The time of the creation of the vacancy with respect to $t=0$ is denoted as $t_{0}$.

If, during the 1 ps of sampling after the introduction of the vacancy defect, the Frenkel defect recombines, a release of approximately $8.9 \mathrm{eV}$ of energy accompanying the recombination event, will be converted into kinetic energy, by virtue of conservation of energy imposed by the micro-canonical ensemble. This increase in kinetic energy will raise the temperature of the system significantly. An example of the evolution of the temperature of the system as a function of time, for one of the simulated Frenkel defects, is shown in Fig. 4.13. The temperature of the interstitial containing system fluctuates around an average of approximately $325 \mathrm{~K}$, during the equilibration period. At $t_{0}$ the vacancy is introduced, creating a Frenkel defect. Approximately $400 \mathrm{fs}$ later, the temperature increases rapidly as a direct result of the recombination of the Frenkel defect. The inflection point of the temperature increase, i.e. the point where the recombination process is at its halfway point is labeled $t_{r}$. We then define the defect lifetime of the Frenkel defect to be $\tau=t_{r}-t_{0}$. To extract this value from the temperature evolution, we define the temperature of the system as a function of time as

$$
T(t)=T_{0}+A e^{\left(t-t_{r}\right) / \sigma} .
$$

Since there are high frequency temperature fluctuations in the raw data, we first take a moving average with a window size of 250 step, or $50 \mathrm{fs}$ and fit Eq.4.2 to the resulting data, using a least-squares optimization algorithm. The computed defect lifetimes for all the simulated Frenkel defects, defined by their vacancy position $V_{x}$, time of creation $t_{0}$ and temperature $T$, are listed in Table 4.3, where a dash indicates that the defect did not recombine throughout the duration of the simulation.

Literature reports high energy particle exposure experiments not just for various boride and energetic particle combinations, such as lithium ion bombarded $\mathrm{B}_{4} \mathrm{C}[80]$ and electron exposed $\mathrm{B}_{12} \mathrm{P}_{2}[15]$, but also for different experimental operating temperatures of $12 \mathrm{~K}[81], 91 \mathrm{~K}$ and $300 \mathrm{~K}[15]$. All these experiments report a similar lack of defect agglomerations and structural damaging, even at lower temperatures, suggesting only a small amount of thermal energy is required to activate the recombination mechanism. Therefore we have performed the Frenkel defect recombination dynamics both at $12 \mathrm{~K}$ and $300 \mathrm{~K}$.

At temperatures of $12 \mathrm{~K}$ and $300 \mathrm{~K}$, just 4 out of 12 Frenkel defects recombine within the $1 \mathrm{ps}$ of the simulation. The recombination pathways corresponded to $I_{a} \rightarrow V_{b}$, $I_{a} \rightarrow V_{d}, I_{b} \rightarrow V_{g}$ and $I_{c} \rightarrow V_{j}$ and did not change their character between runs. The Frenkel defects with a vacancy at either $V_{j}$ or $V_{d}$, consistently recombine within the first $120 \mathrm{fs}$, which confirms the results from the NEB simulations that found that these 


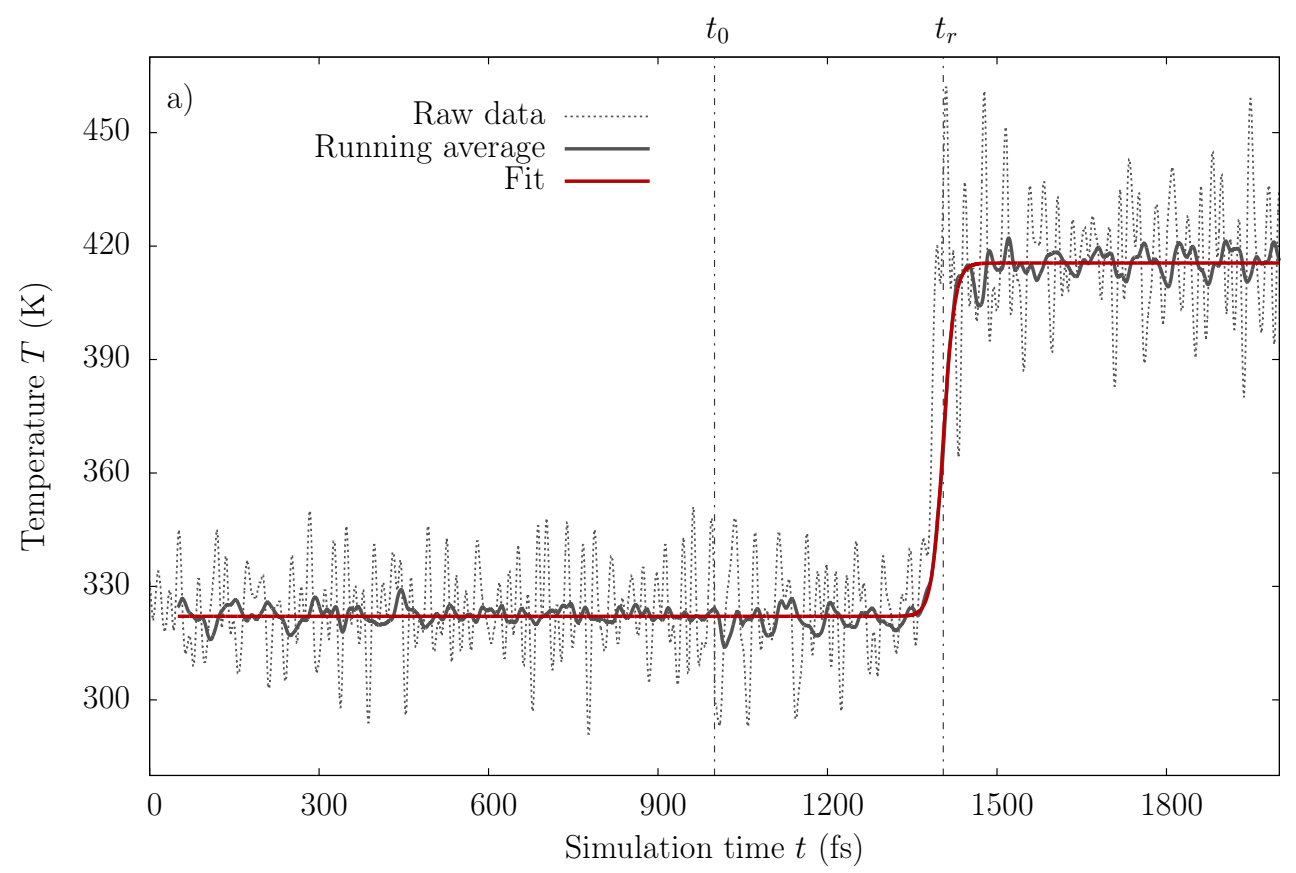

Figure 4.13: Evolution of the temperature during the micro-canonical ensemble sampling of a $\mathrm{B}_{12} \mathrm{P}_{2}$ system that initially includes a single interstitial boron atom. The dashed black line represents the raw temperature as taken from the molecular dynamics trajectory, whereas the solid black line represents a moving average of the former, with a window width of 250 steps, which corresponds to $50 \mathrm{fs}$. The solid red line is a fit of the function defined in Eq. 4.2 to the running average temperature. The first $1000 \mathrm{fs}$ represent the final stage of the equilibration stage with just the interstitial defect, after which at $t_{0}$, marked by a vertical dashed line, a boron vacancy is introduced. The system that now contains a full Frenkel defect is sampled further and after $1400 \mathrm{fs}$ the temperature sharply increases as a direct result of the recombination of the Frenkel defect. The inflection point of the temperature increase, labeled $t_{r}$ and marked by a vertical dashed line, is defined as the time of recombination of the Frenkel defect. 


\begin{tabular}{cccccccccccccc}
\hline & & $V_{a}$ & $V_{b}$ & $V_{c}$ & $V_{d}$ & $V_{e}$ & $V_{f}$ & $V_{g}$ & $V_{h}$ & $V_{i}$ & $V_{j}$ & $V_{k}$ & $V_{l}$ \\
\hline$t_{0}$ & $T$ & & & & \multicolumn{6}{c}{ Defect lifetime $\tau(\mathrm{fs})$} \\
\hline 1 & 12 & - & 202 & - & 114 & - & - & 373 & - & - & 52 & - & - \\
2 & 12 & - & 214 & - & 114 & - & - & 377 & - & - & 53 & - & - \\
3 & 12 & - & 212 & - & 115 & - & - & 370 & - & - & 52 & - & - \\
4 & 12 & - & 304 & - & 117 & - & - & 386 & - & - & 52 & - & - \\
5 & 12 & - & 254 & - & 113 & - & - & 391 & - & - & 52 & - & - \\
\hline 1 & 300 & - & 162 & - & 104 & - & - & 405 & - & - & 58 & - & - \\
2 & 300 & - & 162 & - & 134 & - & - & 335 & - & - & 56 & - & - \\
3 & 300 & - & 927 & - & 125 & - & - & 487 & - & - & 57 & - & - \\
4 & 300 & - & 186 & - & 110 & - & - & 195 & - & - & 65 & - & - \\
5 & 300 & - & 159 & - & 130 & - & - & 152 & - & - & 57 & - & - \\
\hline 1 & 600 & - & 178 & - & 177 & 134 & 199 & 103 & 205 & 236 & 94 & - & - \\
2 & 600 & - & - & - & 121 & - & 183 & - & 214 & - & 143 & - & - \\
3 & 600 & - & - & - & 146 & - & 179 & - & 336 & - & 145 & - & - \\
4 & 600 & - & - & - & 205 & - & 380 & - & 277 & - & 147 & - & - \\
5 & 600 & - & - & - & 127 & - & 413 & - & 373 & - & 129 & - & - \\
\hline
\end{tabular}

TABLE 4.3: The time $\tau$ in fs between the creation of the vacancy defect $t_{0}$ and the recombination of the Frenkel defect for the twelve potential vacancy positions $V_{a}-V_{l}$, where the micro-canonical ensemble of the system is sampled at a temperature $T$ in $\mathrm{K}$. The vacancy creation time $t_{0}$ in ps is referenced with respect to $t=0$ which marks the end of the equilibration of the system containing just the interstitial boron defect.

particular Frenkel defects are not stable and recombine spontaneously. Frenkel defects with their vacancy defect at position $V_{b}$ and $V_{g}$ remained stable considerably longer, but both still recombined well within 1 ps of their creation. This observation confirms the NEB results discussed in the previous section, which predicted a negligible energy barrier for exactly these and only these Frenkel defect recombination pathways.

The NEB method also determined many Frenkel defect recombination pathways with a non-negligible activation energy, all of which did not recombine during the 1 ps of micro-canonical sampling. This is partly due to the fact that the thermal energy $k_{B} T$ at a temperature of $325 \mathrm{~K}$ is approximately $28 \mathrm{meV}$, which is lower than a significant subset of predicted activation energies tabulated in Table 4.2. However, it can also be ascribed to the short simulation time of 1 ps which statistically may prevent the recombination event from being sampled. To illustrate this, we have repeated the same Frenkel defect recombination dynamics at an elevated temperature of $600 \mathrm{~K}$. For the first run, all but 4 out of 12 defects recombine within the first few hundredths of femtoseconds of the simulation, showing that at elevated temperature more defects may recombine in a comparable time frame. However, we only found an increase in the number of Frenkel defects that recombined within the $1 \mathrm{ps}$ of the simulation, for one 
of the runs at $600 \mathrm{~K}$, which highlights that it is not just the available thermal energy but also the sampling duration that determines whether the recombination event will be sampled.

A remarkable difference in defect lifetimes, between the first run and the subsequent four runs at $600 \mathrm{~K}$, is that for the latter, the Frenkel defects with a vacany at position $V_{b}$ and $V_{g}$ and a finite lifetime at lower temperatures, no longer recombined. Similarly, defects with the vacancy at $V_{f}$ and $V_{h}$, which never recombined at $12 \mathrm{~K}$ and $300 \mathrm{~K}$, all of a sudden had a finite lifetime smaller than $1 \mathrm{ps}$. The explanation for this observation is not that the elevated temperature enable a new recombination mechanism, but rather, during the equilibration period at $600 \mathrm{~K}$, the system underwent polar diffusion of the interstitial complex. We described this process in Section 4.3.5, where the icosahedral pole containing the interstitial boron atom, changes its orientation with respect to its neighboring icosahedra. The activation energy of this skewing motion is relatively low at $0.2 \mathrm{eV}$ [see Fig. 4.7] and a closer inspection of the equilibration trajectory of the system at $600 \mathrm{~K}$ revealed that the polar interstitial diffusion occurred roughly every picosecond. At the moment that the vacancies were introduced for the first run at $600 \mathrm{~K}$, the system was just transitioning from the original interstitial orientation, on which the labeling in Fig. 4.10 is based, to another orientation. The apparent change in which defects recombine is therefore merely a result of the changed orientation of the interstitial pole that transforms the original labeling, which can be undone by a symmetrical transformation. The recombination pathways for runs 2 to 5 at $600 \mathrm{~K}$ with a vacancy at the positions $V_{f}$ and $V_{h}$, correspond to the mediated $I_{a} \rightarrow V_{b}$ pathway visualized in Fig. 4.11. Similarly, defects labeled $V_{d}$ and $V_{j}$ had a recombination pathway that can be mapped to $I_{a} \rightarrow V_{d}$ on the original labeling. The fact that the system was in a structural transition at the moment of the first run at $600 \mathrm{~K}$, also explains why more Frenkel defects recombined, since the structure was temporarily even further removed from its equilibrium position, opening access to other recombination channels.

Generally, the findings of the simulated dynamics of the Frenkel defects agree with the predicted activation energies for their recombination, presented in Section 4.3.6. Defects with a negligible activation energy all recombined within 1 ps of the molecular dynamics simulation and the other defects all remained in tact, in agreement with their predicted non-negligible activation energy and the relatively short simulation time of 1 ps. However, there is one particular defect that stands out. The NEB method predicted an activation energy of only $40 \mathrm{meV}$ for the $I_{a} \rightarrow V_{a}$ recombination pathway, yet in none of the molecular dynamics simulations did this defect recombine. This defect then provides the perfect example to reiterate that it is not just the activation energy that determines whether a predicted recombination pathway will be sampled, but rather the specific motion required by that pathway may be unlikely to be sampled. Like the recombination of $I_{a} \rightarrow V_{b}$, as illustrated in Fig. 4.11, the recombination pathway for $I_{a} \rightarrow V_{a}$ is a mediated recombination. However, unlike in the case of 
a)

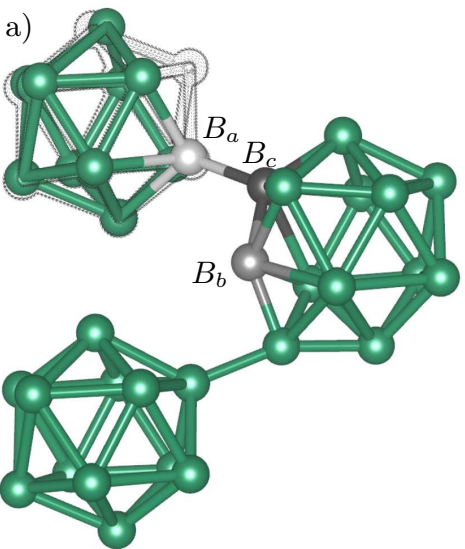

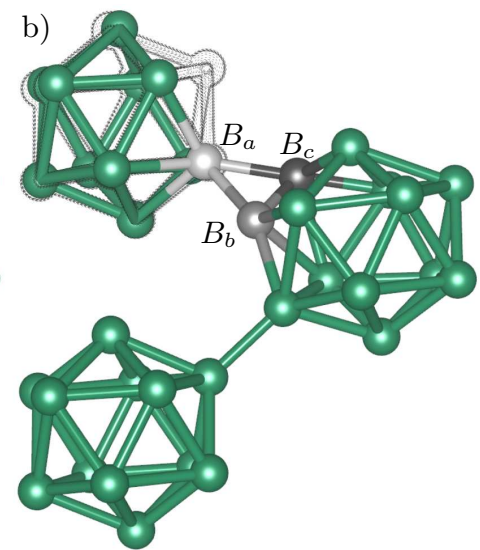

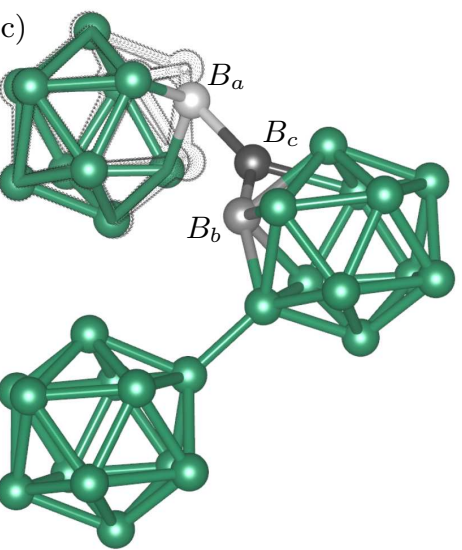

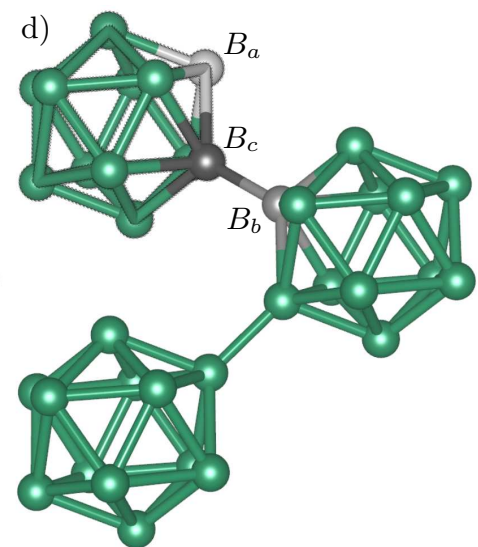

Figure 4.14: (a-d) Partial structures of the initial, third, fifth and final image, respectively of the relaxed recombination pathway for the $I_{a} \rightarrow V_{a}$ Frenkel defect. The boron atoms that are the main participants in the diffusive recombination are labeled $B_{a}, B_{b}$ and $B_{c}$, and are represented by grey spheres, going from light to dark, respectively. All four images show a black mesh outline over the top left vacancy containing icosahedron, which traces the atoms of the icosahedron in its relaxed configuration in the final image. This serves as a guide to the eye, to indicate the relaxed atomic positions that the diffusing atoms will end up in. 
$I_{a} \rightarrow V_{b}$, where the diffusive motion is limited to just two atoms that move in a similar direction for the entire pathway, the NEB determined recombination pathway for $I_{a} \rightarrow V_{b}$ hinges on the collaborative movement of at least three atoms, whose motion changes direction multiple times. This coordinated movement of multiple atoms in the predicted recombination pathway $I_{a} \rightarrow V_{a}$ is visualized in Fig. 4.14.

For this recombination to occur, first atom $B_{b}$ has to move a significant distance towards $B_{a}$ and $B_{c}$ to form a threefold coordinated boron complex. Then a vacancy diffusion is required where $B_{a}$ diffuses to the position $V_{a}$, while $B_{b}$ and $B_{c}$ remain mostly stationary. The recombination is completed finally by a motion of $B_{c}$ that is directly perpendicular to the vacancy diffusion of $B_{a}$. The rather complicated nature of this sequence of events, characterized by motions that are mostly perpendicularly directed and nonsimultaneous, explains why the pathway that is defined by it is not necessarily sampled, despite having a low activation energy.

\subsubsection{FRENKEL DEFECT CHARGE LOCALIZATION}

To determine the charge density localization associated with the presence of a Frenkel defect, we have performed Bader analysis on the 10 stable Frenkel defects, which will yield an effective charge for each atom in the cell[68]. The computed effective valencies for the atoms on the vacancy containing icosahedron and the atoms on the interstitial containing icosahedral pole, are tabulated in Table 4.4 .

\begin{tabular}{lcccccc}
\hline Vacancy & $Q_{\text {vac }}$ & $Q_{a}$ & $Q_{b}$ & $Q_{c}$ & $Q_{d}$ & $Q_{\text {int }}$ \\
\hline$V_{a}$ & 2.86 & 2.80 & 3.66 & 3.17 & 2.80 & 3.11 \\
$V_{b}$ & 2.88 & 2.69 & 4.02 & 2.90 & 2.89 & 3.12 \\
$V_{c}$ & 2.87 & 2.83 & 4.01 & 2.88 & 2.81 & 3.13 \\
$V_{e}$ & 2.89 & 2.80 & 3.34 & 3.63 & 2.79 & 3.14 \\
$V_{f}$ & 2.89 & 2.82 & 3.88 & 3.08 & 2.79 & 3.14 \\
$V_{g}$ & 2.90 & 2.94 & 2.84 & 3.97 & 2.95 & 3.17 \\
$V_{h}$ & 2.91 & 2.87 & 3.35 & 3.41 & 2.91 & 3.14 \\
$V_{i}$ & 2.91 & 2.91 & 3.34 & 3.41 & 2.88 & 3.13 \\
$V_{k}$ & 2.86 & 2.91 & 2.99 & 3.71 & 2.87 & 3.12 \\
$V_{l}$ & 2.86 & 2.86 & 3.00 & 3.74 & 2.91 & 3.13 \\
\hline
\end{tabular}

TABLE 4.4: Effective number of valence electrons for various boron atoms in a supercell containing a stable Frenkel defect, as obtained from Bader analysis. The column labeled $Q_{\mathrm{vac}}$ gives the average valency of all boron atoms on the vacancy containing icosahedron. The columns labeled $Q_{a}$ through $Q_{d}$ represents the valency of the boron atoms on the interstitial containing icosahedron, labeled $I_{a}$ through $I_{d}$ in Fig. 4.15, respectively. The final column $Q_{\text {int }}$ corresponds to the average of the four columns directly preceding it. 


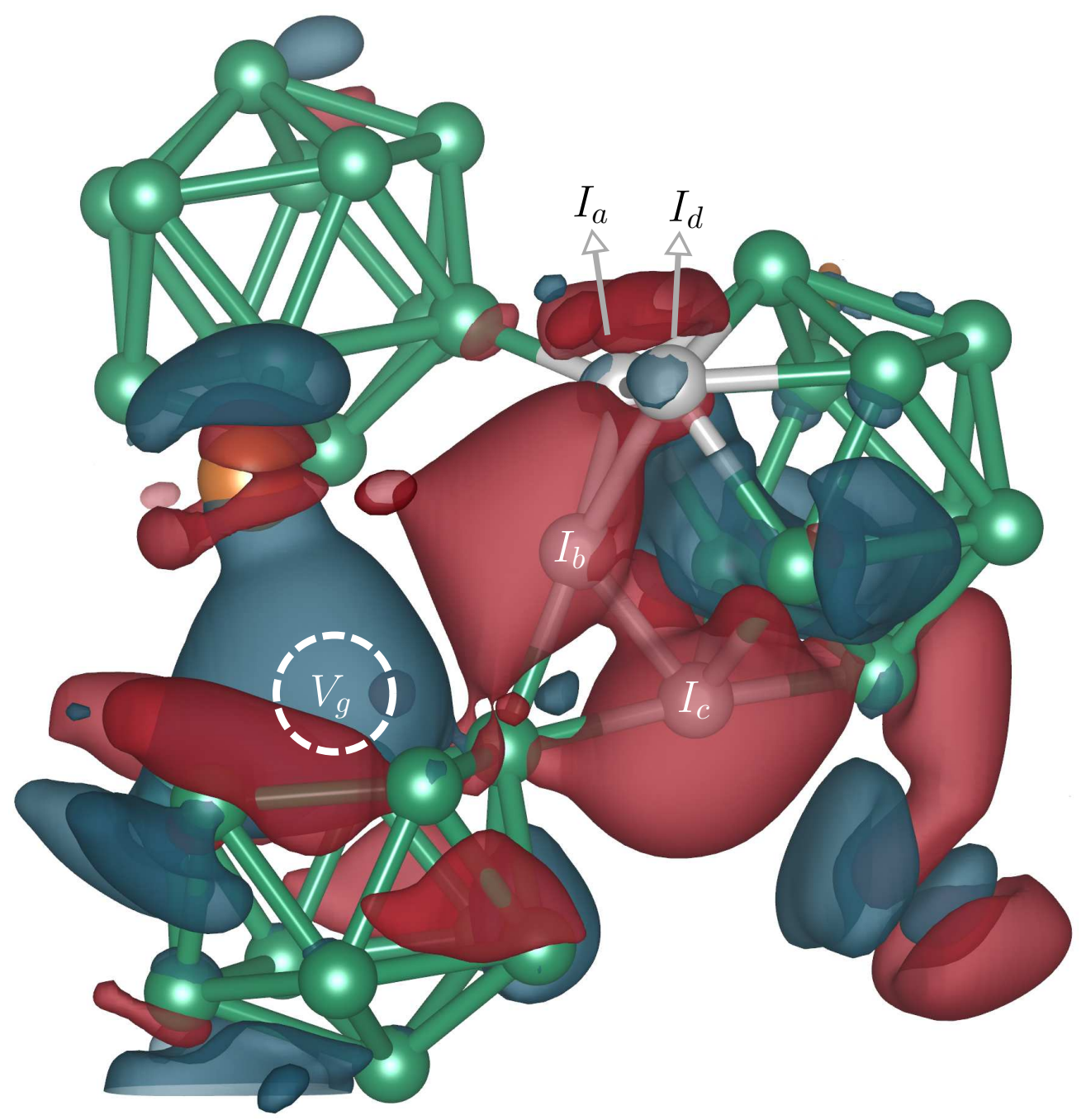

FiguRE 4.15: Isosurface visualization of the electron density difference between the $\mathrm{B}_{12} \mathrm{P}_{2}$ supercell containing a Frenkel defect with a vacancy at position $V_{g}$ and the perfect crystal. The red and blue colors of the isosurfaces indicate where the electron density of the defective cell is larger and smaller compared to the bulk, respectively. In other words, locations in red (blue) show regions of the defective cell that have a net larger (smaller) electronic density compared to the bulk and therefore have a net negative (positive) charge. The specific Frenkel defect pictured has a vacancy at position $V_{g}$, indicated by the dashed white circle. The boron atoms on the icosahedron containing the interstitial defect are labeled $I_{a}$ through $I_{d}$, whose corresponding effective valence charges as derived from Bader analysis, are displayed in Table 4.4 under the columns labeled $Q_{a}$ through $Q_{d}$, respectively 
Bader analysis shows that the boron atoms on the vacancy containing icosahedron will have a net charge of approximately +0.1 , resulting in an overall +1 charge for the corresponding icosahedron. The electrons given up by the vacancy containing icosahedron are mostly transferred to the boron atoms of and directly adjacent to the interstitial defect. When the vacancy resides on the bottom left icosahedron in Fig. 4.10, atom $I_{c}$ has the largest net electronic charge varying from -0.97 to -0.40 . Conversely, for a Frenkel defect with its vacancy on the upper left icosahedron in Fig. 4.10, it is the atom labeled $I_{b}$ that garners the largest charge ranging from -1.02 to -0.34 . In general, for any Frenkel defect, there is a large charge transfer from the vacancy containing icosahedron to the interstitial containing icosahedral pole, where the former and latter icosahedron effectively carry a total charge of +1 and -1 . To visualize this charge transfer, we have computed the charge density difference between the supercell containing a Frenkel defect and the perfect crystal. An isosurface representation of the charge density difference for the $V_{g}$ Frenkel defect is shown in Fig. 4.15. The combination of an large effective charge difference of approximately $2 e$ over a short distance, leads to a large Coulomb interaction which is the driving force behind the recombination process.

\subsubsection{CHARGED FRENKEL DEFECTS}

The previous section highlighted that a Frenkel defect is accompanied by an intrinsic charge transfer from the vacancy containing icosadron to the interstitial containing icosahedron, but the overall charge of the system was maintained to be neutral, as it has been for all calculations discussed up till now. In reality however, structural defects, especially in semiconductors like $\mathrm{B}_{12} \mathrm{P}_{2}$, can exist in various charge states, which could potentially change the described defect recombination behaviour. Repeating all the calculations presented so far, for all possible charge states of the defect, would be an enormouns and inefficient undertaking and moreover beyond the scope of this work. However, we can not leave the discussion about the potential influence of charged defects on the presented defect recombination mechanism untouched.

As a compromise, we have repeated the dynamics simulations, as presented for the neutral system in Section 4.3.7, for various charge states of the system at a temperature of $300 \mathrm{~K}$. Defects created in semiconductors due to electron irradiation, as in the case of the experiment whose observation of self-healing we are trying to model[15], are usually left in a positive charge state due to an overall depletion of electrons[82]. Therefore, we consider the 12 possible Frenkel defects as described in Section 4.3.7, where the entire cell has an effective charge of $+1,+2$ or +3 . We reduced the number of runs for each unique configuration from 5 to 3 . The computed recombination times from the molecular dynamics trajectories are shown in Table 4.5, which also include the corresponding results of the neutral cell of Table 4.3 at the same temperature for comparison. 


\begin{tabular}{cccccccccccccc}
\hline & & $V_{a}$ & $V_{b}$ & $V_{c}$ & $V_{d}$ & $V_{e}$ & $V_{f}$ & $V_{g}$ & $V_{h}$ & $V_{i}$ & $V_{j}$ & $V_{k}$ & $V_{l}$ \\
\hline$t_{0}$ & $Q$ & & & & \multicolumn{6}{c}{ Defect lifetime $\tau(\mathrm{fs})$} \\
\hline 1 & 0 & - & 162 & - & 104 & - & - & 405 & - & - & 58 & - & - \\
2 & 0 & - & 162 & - & 134 & - & - & 335 & - & - & 56 & - & - \\
3 & 0 & - & 927 & - & 125 & - & - & 487 & - & - & 57 & - & - \\
\hline 1 & +1 & - & 258 & - & 106 & - & - & 127 & - & - & 57 & 562 & 222 \\
2 & +1 & - & 684 & - & 142 & - & - & 357 & - & 391 & 56 & - & - \\
3 & +1 & - & - & - & 133 & - & - & 150 & - & - & 58 & 362 & - \\
\hline 1 & +2 & 364 & - & - & 128 & - & - & 121 & - & 230 & 59 & 98 & 95 \\
2 & +2 & 325 & 779 & - & 224 & - & - & 392 & - & 485 & 59 & 113 & 110 \\
3 & +2 & 301 & - & - & 178 & - & - & 136 & - & 509 & 62 & 131 & 121 \\
\hline 1 & +3 & 306 & - & - & 189 & - & - & 147 & 506 & 938 & 62 & 100 & 97 \\
2 & +3 & 786 & - & - & 858 & - & - & 494 & 987 & - & 62 & 114 & 111 \\
3 & +3 & 333 & - & - & 179 & - & - & 457 & 606 & - & 64 & 135 & 124 \\
\hline
\end{tabular}

TABLE 4.5: The time $\tau$ in fs between the creation of the vacancy defect $t_{0}$ and the recombination of the Frenkel defect for the twelve potential vacancy positions $V_{a}-V_{l}$, where the micro-canonical ensemble of the system is sampled at a temperature of $300 \mathrm{~K}$ and the overall cell has an effective charge $Q$. The vacancy creation time $t_{0}$ in ps is referenced with respect to $t=0$ which marks the end of the equilibration of the system containing just the interstitial boron defect.

The recombination times of three of the four defects which consistently recombined within $1 \mathrm{ps}$ in a charge neutral system, i.e. $V_{d}, V_{g}$ and $V_{j}$, remained comparable when a net positive charge was introduced to the system, indicating that the overall charge of the cell had little influence on the recombination dynamics. Contrastingly, the defect with a vacancy at $V_{b}$, which consistently recombined for a neutral system, remained stable for some of the runs of the positively charged cell. Interestingly, the exact opposite behavior was observed for defect $V_{a}$, which shares similaries with $V_{b}$ in terms of the orientation of the interstitial defect with respect to the vacancy. Additionally, several defects, $V_{h}, V_{i}, V_{k}$ and $V_{j}$, which were rarely observed to recombine for $Q=0$, now all regularly recombined for $Q>0$. In the way we verified the predicted recombination pathways through NEB calculations by explicitly running molecular dynamics in the first part of this work, we now reverse that process and further investigate the newly observed recombination dynamics of certain Frenkel defects by explicitly mapping the recombination pathway with the NEB method.

Since the vacancy pairs $V_{h}-V_{i}$ and $V_{k}-V_{l}$ are symmetrically equivalent, we have limited computing the recombination pathway for Frenkel defects in a charged cell with a vacancy at $V_{a}, V_{i}$ or $V_{k}$. The molecular dynamics trajectories showed that all recombination events were mediated recombinations of $I_{a}$ or $I_{c}$, except for $V_{i}$ where the 


\begin{tabular}{lcccc}
\hline & \multicolumn{3}{c}{$\Delta E_{a}$} \\
\hline Pair & $Q=0$ & $Q=+1$ & $Q=+2$ & $Q=+3$ \\
\hline$I_{b} \rightarrow V_{i}$ & 0.31 & 0.11 & 0.07 & 0.06 \\
$I_{c} \rightarrow V_{i}$ & 0.16 & 0.15 & 0.02 & $0.00^{*}$ \\
$I_{a} \rightarrow V_{a}$ & 0.04 & 0.05 & $0.00^{*}$ & 0.009 \\
$I_{c} \rightarrow V_{k}$ & 0.68 & 0.03 & - & - \\
\hline
\end{tabular}

TABLE 4.6: Diffusion activation energies $\Delta E_{a}$ of recombination pathways of various Frenkel pairs in $\mathrm{eV}$. The interstitial and vacancy labels of the Frenkel pairs directly correspond to the labels in Fig. 4.10. The activation energies equal to zero (marked by an asterisk) correspond to recombination pathways that were monotonically decreasing in energy for the original number of images. Since a meta-stable initial image was found for these Frenkel configurations, the energy maximum had to be located between the first two images, however, even after adding up to 8 images in between these first two images, a well-defined energy maximum still could not be found. This implies that the true activation energy for these recombination pathways is on the order of $1 \mathrm{meV}$.

direct recombination through $I_{b}$ was also observed. Therefore we have computed the recombination pathways $I_{b} \rightarrow V_{i}, I_{c} \rightarrow V_{i}, I_{a} \rightarrow V_{a}$ and $I_{c} \rightarrow V_{k}$ for systems with a net charge $Q$ of +1 to +3 . The obtained activation energies for the recombination pathways are shown in Table 4.6.

For all recombination pathways, the activation energy, almost unilaterally, decreases as the net charge of the system becomes more positive. This decrease in activation energy for these particular recombination pathways, explains why the defects recombined in the molecular dynamics simulations of the positively charged cells, but remained stable in the neutral case. In conclusion, the charge state of Frenkel defects does clearly affect the recombination dynamics, however, the nature of the recombination mechanism, the mediated diffusion of vacancy and interstitial boron defects, remains unchanged compared to neutral defects. The charge state of the defect merely changes the local free energy landscape which determines which recombination pathways are energetically accessible.

\subsection{CONCLUSIONS}

In this work, we have investigated the static structural properties of boron vacancy and interstitial point defects in the icosahedral boride $\mathrm{B}_{12} \mathrm{P}_{2}$ and found that degraded icosahedra that contain either a vacancy or an interstitial defect, remain stable and maintain their icosahedral structure. Using nudged elastic band calculations, the mobility of vacancy and interstitial defects was studied, which revealed that the majority 
of intra-icosahedral diffusion pathways of both defect types have a high activation energy barrier and are unlikely to occur, with the notable exception of the polar diffusion of an interstitial boron defect of type $\mathrm{I}_{\mathrm{B}}^{\mathrm{A}}$.

Following the study of vacancy and interstitial mobility individually, the mobility of a combination of these two point defects in the form of a Frenkel defect pair, was investigated. We have shown that all Frenkel configurations have a (meta-)stable state, except when the vacancy is located on the boron site that is directly bound to icosahedral face containing the interstitial boron atom, in which case the Frenkel defect will spontaneously recombine. For all other considered Frenkel pairs, various possible recombination pathways were modeled with the nudged elastic band method, which showed that certain Frenkel defects had an activation energy for recombination as low as $3 \mathrm{meV}$. The surprisingly low energy barrier is the cumulative result of two diffusion processes operating synchronously, where the inter-icosahedral diffusion of the interstitial boron is mediated by the intra-icosahedral vacancy diffusion of another boron atom. The Frenkel defect recombination pathways determined with the NEB method, have been verified by explicitly running molecular dynamics for the various defect configurations. The extracted lifetimes from these trajectories agreed to a high degree with the predicted activation energies.

We have also shown that the particular charge state of the defect does affect the recombination behavior, however, it does not alter the fundamental mediating character underlying the recombination mechanism. The charge state of the defect merely influences the local free energy landscape that makes certain recombination pathways energetically more easily accessible, while making others energetically more costly, increasing the Frenkel defect's effective lifetime. The demonstration of the mediated recombination mechanism for Frenkel defects, with activation energies of the order of just a few $\mathrm{meV}$, corroborate earlier experimental results on the "self-healing" properties of $\mathrm{B}_{12} \mathrm{P}_{2}$.

\section{ACKNOWLEDGMENTS}

This work is supported by NanoNextNL, a micro and nanotechnology programme of the Dutch Government and 130 partners. We acknowledge the support of the Center for X-ray Optics of Lawrence Berkeley Laboratory and the Industrial Focus Group XUV Optics at the MESA+ Institute for Nanotechnology at the University of Twente, notably the partners ASML, Carl Zeiss SMT GmbH, and the Foundation FOM. All the computational work was performed at the Molecular Foundry which is supported by the Office of Science, Office of Basic Energy Sciences, of the United States Department of Energy under Contract No. DE-AC02-05CH11231. 


\section{REFERENCES}

[15] M. Carrard, D. Emin, and L. Zuppiroli, Phys. Rev. B 51, 11270 (1995).

[16] D. Emin, Journal of Solid State Chemistry 179, 2791 (2006).

[17] J. C. Lund, F. Olschner, F. Ahmed, and K. S. Shah, MRS Proc. 162 (1989) 10.1557/proc-162-601.

[18] T. P. Viles, B. A. Brunett, H. Yoon, J. C. Lund, H. Hermon, D. Buchenauer, K. McCarty, M. Clifft, D. Dibble, and R. B. James, MRS Proc. 487 (1997) 10.1557/proc-487-585.

[47] G. Kresse, and J. Furthmüller, Physical Review B 54, 11169 (1996).

[61] P. E. Blöchl, Physical Review B 50, 17953 (1994).

[62] G. Kresse, and D. Joubert, Physical Review B 59, 1758 (1999).

[63] J. P. Perdew, K. Burke, and M. Ernzerhof, Phys. Rev. Lett. 77, 3865 (1996).

[65] H.-P. Komsa, T. T. Rantala, and A. Pasquarello, Physical Review B 86, 045112 (2012).

[68] W. Tang, E. Sanville, and G. Henkelman, Journal of Physics: Condensed Matter 21, 084204 (2009).

[69] Q. An, and W. A. Goddard, Chemistry of Materials 27, 2855 (2015).

[70] Q. An, K. M. Reddy, H. Dong, M.-W. Chen, A. R. Oganov, and W. A. Goddard, Nano Letters (2016) 10.1021/acs.nanolett.6b01204.

[71] G. Henkelman, B. P. Uberuaga, and H. Jónsson, The Journal of Chemical Physics 113, 9901 (2000).

[72] G. Henkelman, and H. Jónsson, The Journal of Chemical Physics 113, 9978 (2000).

[73] H. J. Monkhorst, and J. D. Pack, Phys. Rev. B 13, 5188 (1976).

[74] P. A. Schultz, Phys. Rev. Lett. 84, 1942 (2000). 
[75] M. Leslie, and N. J. Gillan, J. Phys. C: Solid State Phys. 18, 973 (1985).

[76] G. Makov, and M. C. Payne, Phys. Rev. B 51, 4014 (1995).

[77] S. Lany, and A. Zunger, Phys. Rev. B 78 (2008) 10.1103/physrevb.78.235104.

[78] C. Freysoldt, J. Neugebauer, and C. G. V. de Walle, Phys. Rev. Lett. 102 (2009) 10.1103/physrevlett.102.016402.

[79] T. L. Aselage, D. R. Tallant, and D. Emin, Phys. Rev. B 56, 3122 (1997).

[80] T. Stoto, N. Housseau, L. Zuppiroli, and B. Kryger, J. Appl. Phys. 68, 3198 (1990).

[81] T. Stoto, L. Zuppiroli, and J. Pelissier, Radiation Effects 90, 161 (1985).

[82] O. Cretu, Y.-C. Lin, and K. Suenaga, Micron 72, 21 (2015). 


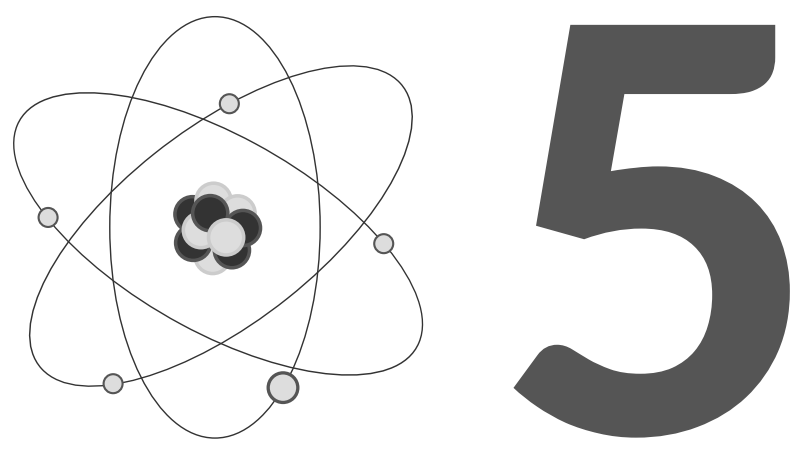

DETECTION OF DEFECT POPULATIONS IN SUPERHARD SEMICONDUCTOR BORON SUBPHOSPHIDE $\mathrm{B}_{12} \mathrm{P}_{2}$ THROUGH X-RAY ABSORPTION SPECTROSCOPY 


\section{ABSTRACT}

To study the quality of synthetic samples of the superhard semiconductor boron subphosphide, the x-ray spectroscopic signatures of potential defect populations in $\mathrm{B}_{12} \mathrm{P}_{2}$ samples are predicted and compared with measured x-ray absorption spectra. The boron $K$-edge and phosphorus $L_{2,3}$-edge spectroscopy is collected experimentally for a set of $\mathrm{B}_{12} \mathrm{P}_{2}$ samples deposited under varying experimental conditions. Employing calculations from first-principles, the experimentally collected spectra are simulated within the density functional theory framework. Spectroscopic fingerprinting of potential crystallographic point defects, permits the determination of the presence of certain defects. Our results find an increasing propensity of phosphorus vacancy defects for samples deposited at lower temperatures but no evidence for comparable populations of boron vacancies in all the samples that have been studied. The absence of large amounts of boron vacancies may be connected to the reported "self-healing" properties of $\mathrm{B}_{12} \mathrm{P}_{2}$. 


\subsection{INTRODUCTION}

The boron-rich solid $\mathrm{B}_{12} \mathrm{P}_{2}$, also known as boron subphosphide or icosahedral boron phosphide, has been the subject of a variety of research studies, due to its interesting properties including high (radiation) hardness[83], chemical inertness and neutron capture cross-section[17]. The combination of these properties make it an ideal candidate for applications in for example radiation shielding, neutron detectors and betavoltaic cells[16-18]. One of the more intriguing properties of this material, is the alledged observation of a non-thermally driven "self-healing" mechanism. Transmission electron microscopy of $\mathrm{B}_{12} \mathrm{P}_{2}$ samples, after prolonged exposure to high energy particles, showed no visible damage patterns[15]. Under the conditions of the experiment, in terms of radiation energy and exposure time, it was estimated that each and every boron atom had to have been displaced on the order of 10 times, which, following the lack of observed structural damage, led to the suggestion of an intrinsic "self-healing" property of the material[15, 16]. The lack of structural damage was observed in experiments conducted both at room and lowered temperatures of $12 \mathrm{~K}$, suggesting a non-thermally driven mechanism underlying the restorative quality of $\mathrm{B}_{12} \mathrm{P}_{2}$.

In a recent publication[84], the proposed mechanism was investigated theoretically, where the static structural properties of vacancy and interstitial defects and the dynamical process of defect recombination were simulated within the density functional theory (DFT) framework. The calculations revealed that the boron icosahedra, that form the cornerstone of the $\mathrm{B}_{12} \mathrm{P}_{2}$ crystal structure, remain structurally stable, even in the presence of a vacancy or interstitial defect. However, for certain configurations of an interstitial and vacancy defect pair (a Frenkel defect), the activation barrier for defect recombination can be almost negligible. This surprisingly low energy barrier would be surmountable even at low temperatures and could potentially explain the experimentally observed "self-healing" in $\mathrm{B}_{12} \mathrm{P}_{2}$.

An experimental verification, of the theoretically proposed static defect characteristics and the properties of the dynamic recombination process, is now required. $\mathrm{X}$ ray absorption near-edge spectroscopy (XANES) is sensitive to structural defects in borides $[1,57-60]$ and could potentially be used to monitor defect formation and defect abundances during sample irradiation. However, to the best of the authors' knowledge, $\mathrm{x}$-ray spectroscopy data for $\mathrm{B}_{12} \mathrm{P}_{2}$ has not yet been reported in literature and therefore a reference signature of pristine $\mathrm{B}_{12} \mathrm{P}_{2}$ is not available. We will present experimental x-ray absorption spectroscopy measurements of the boron $K$-edge and phosphorus $L_{2,3}$-edge for several $\mathrm{B}_{12} \mathrm{P}_{2}$ samples deposited with chemical vapor deposition under varying conditions.

A theoretical analysis of the collected spectra is conducted from first-principles within the DFT framework, which provides a spectroscopic fingerprint for crystalline $\mathrm{B}_{12} \mathrm{P}_{2}$. Additionally, first-principles calculations are employed to simulate the spectroscopic signatures of potential point defects in $\mathrm{B}_{12} \mathrm{P}_{2}$, which are used to discuss the abundances 
of these defects present in the analyzed samples. These defect spectroscopy signatures could be utilized in future studies collecting x-ray absorption spectroscopy measurements of samples during irradiation by high energy particles, to observe the evolution of defect populations in situ. The experimental spectroscopic results presented in this work, indicate a lack of boron vacancies, in line with the findings of earlier work on the "self-healing" of $\mathrm{B}_{12} \mathrm{P}_{2}$, which would predict a lack of boron vacancies by definition.

\subsection{EXPERIMENTAL DETAILS}

All x-ray absorption spectroscopy measurements were carried out at beamline 6.3 .2 of the Advanced Light Source (ALS) synchrotron at Lawrence Berkeley National Laboratory (LBNL). A detailed description and characterization of the beamline and measurement chamber can be found elsewhere[42, 43]. X-ray absorption measurements of the boron $K$-edge and phosphorus $L_{2,3}$ edge, were collected in total electron yield (TEY) mode. The linearly polarized incident soft x-ray beam was oriented parallel to the sample surface normal. Energy calibration was performed by comparing to absolute absorption edges of $\mathrm{Si}$ and $\mathrm{B}$ filters installed at the beamline. The collected spectra have the dark current signal subtracted to account for the systematic error and noise in the collector electronics. Subsequently, the spectra are normalized by a spectrum collected by a photodiode to account for the intensity fluctuations in the x-ray beam as a function of photon energy.

\subsection{COMPUTATIONAL METHOD}

\subsubsection{STRUCTURAL RELAXATIONS}

All structural optimizations have been carried out within the DFT framework using the Vienna $a b$ initio simulation package VASP[47]. Core electrons are replaced by ultrasoft pseudopotentials within the projector augmented wave (PAW) method[61, 62] where $2 s$ and $2 p$ electrons for boron and, $3 s$ and $3 p$ electrons for phosphorus are treated as valence electrons. The generalized gradient approximation (GGA) as formulated by Perdew-Burke-Ernzerhof[63] (PBE) is employed for the exchange-correlation energy. A $2 \times 2 \times 2$ supercell from the $\mathrm{B}_{12} \mathrm{P}_{2}$ unitcell was used for all calculations, totalling 336 boron and 48 phosphorus atoms for the perfect crystalline supercell. Plane waves were expanded up to an energy of $400 \mathrm{eV}$ and the Brillouin zone was sampled at the $\Gamma$

point. In calculations of total energies, for the purpose of computing defect formation energies, the $k$-point density was increased to a $2 \times 2 \times 1$ Monkhorst-Pack mesh[73], to ensure convergence. In density of states calculations the Brillouin zone integration was performed with the tetrahedron method[85], over a $8 \times 8 \times 4$ Monkhorst-Pack mesh 
and, where applicable, for screened hybrid functionals the HSE06 functional was employed[86]. Thermally induced structural distortions of the bulk $\mathrm{B}_{12} \mathrm{P}_{2}$ crystal lattice were simulated by sampling the canonical ensemble $(N V T)$ at a finite temperature of $300 \mathrm{~K}$ regulated by a Nosé-Hoover thermostat. The time step of integration was set to $0.2 \mathrm{fs}$. The system was thermally equilibrated for $5 \mathrm{ps}$, after which the sampling was switched to the micro canonical ensemble $(N V E)$ maintaining a temperature of $300 \mathrm{~K}$. From this trajectory three snapshots were taken at intervals of $2.5 \mathrm{ps}$ for which the average $\mathrm{x}$-ray absorption spectrum was calculated, to represent a statistical average of the structure at finite temperature.

\subsubsection{FORMATION ENERGIES}

As an estimate for the likelihood of formation of any of the considered point defects we compute the formation energy $E^{f}$, which for a defect $X$ in charge state $q$ is commonly estimated by the equation[65]

$$
\begin{aligned}
E^{f}\left[X^{q}\right]=E\left[X^{q}\right]+E_{\mathrm{corr}}^{q} & -E[\text { host }]-\sum n_{i} \mu_{i} \\
& +q\left(\epsilon_{F}+\epsilon_{v}+\Delta v\right),
\end{aligned}
$$

where $E\left[X^{q}\right]$ and $E[$ host $]$ are the total energies of the defective and pristine host structures, respectively, as derived from supercell calculations. The amount of particles of type $i$, both native and impure, that are added or removed is given by $n_{i}$, which is positive for a net amount of particles added and negative otherwise. Assuming thermodynamic equilibrium, the native and impurity atoms are assumed to be exchanged with a reservoir at a chemical potential given by $\mu_{i}$ which can be related through an equilibrium definition and the chemical potentials of a boron and phosphorus solid phase. In the limit of a boron- or phosphorus rich environment, the corresponding chemical potential can then be computed from the Gibbs free energy from the boron $\alpha$-rhombohedral or $\mathrm{B}_{12} \mathrm{P}_{2}$ bulk phase, respectively, thereby fixing the chemical potential of the other elemental species one through the equilibrium definition. All defect calculations were performed for the neutral charge state and therefore the image-charge correction term $E_{\text {corr }}^{q}$ and the potential alignment term $\Delta v$ can be ignored[65, 66].

\subsubsection{X-RAY ABSORPTION SPECTROSCOPY}

X-ray absorption spectroscopy (XAS) was simulated within the density functional theory excited core-hole (DFT-XCH) approach[36] where the photo excited atom is modeled by removing a core electron from the pseudopotential and placing it in the first available empty state. The electronic structure problem of the system that now includes the core-hole is then solved self-consistently under constrained occupations while employing the Shirley interpolation scheme[49] to generate optimal basis sets in order to reduce computational cost. The absorption spectrum is computed by evaluating the 
transition probability between the initial and final state as given by Fermi's golden rule within the dipole approximation. The resulting spectrum is broadened uniformly with a Gaussian of $0.2 \mathrm{eV}$ at FWHM. To correct for the well-known underestimation of the band gap by the PBE functional, the energy scale is stretched uniformly by a factor of 1.08 for the boron $K$-edge and 1.06 for the phosphorus $L_{2,3}$-edge. Due to the lack of an absolute energy reference inherent in the pseudopotential method, an energy alignment scheme was employed to yield comparably meaningful relative energies for structurally and chemically different systems[67]. Finally, the entire spectrum is shifted by a single value, which is kept constant for all computed spectra, to align with the experimental data. In the case of a $L_{2,3}$-edge transition, electrons from either the $2 p_{3 / 2}$ or $2 p_{1 / 2}$ core level can be excited, which, due to spin-orbit coupling, are energetically split. Under the assumption that excitations from $2 p_{3 / 2}$ or $2 p_{1 / 2}$ core levels are equally probable, in this work, this effect is accounted for by adding the spectrum to itself, after shifting it by an energy $\Delta E$ equal to the spin-orbit splitting of the relevant element, and properly weighting the two spectra by a ratio of $2: 1$ that reflects the population ratio of the $2 p_{3 / 2}$ and $2 p_{1 / 2}$ core levels.

\subsection{EXPERIMENTAL RESULTS}

In this section, we will report on the experimental details of sample deposition, experimental sample characterization and boron $K$-edge and phosphorus $L_{2,3}$-edge x-ray absorption spectroscopy, performed at beamline 6.3.2 at the Advanced Light Source (ALS).

\subsubsection{SAMPLE DEPOSITION}

Multiple samples of $\mathrm{B}_{12} \mathrm{P}_{2}$ were grown on $4 H$-SiC substrates in a cold-walled, inductively heated chemical vapor deposition (CVD) reactor, a detailed description of which can be found elsewhere[87]. The substrates were placed in a TaC-coated graphite susceptor, where they were heated and etched in a $\mathrm{H}_{2}$ carrier gas for 20 min. Sample L1 was etched at a temperature of $1650^{\circ} \mathrm{C}$ and the other samples were etched at a lower temperature of $1250{ }^{\circ} \mathrm{C}$. The carrier gas was maintained at $3500 \mathrm{sccm}$ for all experiments. After the etching process was completed, the substrates were brought to the deposition temperature, which ranged from $1050{ }^{\circ} \mathrm{C}$ to $1250{ }^{\circ} \mathrm{C}$. The $\mathrm{B}_{2} \mathrm{H}_{6}\left(1 \% \mathrm{~B}_{2} \mathrm{H}_{6}\right.$ in $\mathrm{H}_{2}$ balance) flow rate was held constant at 40 or $60 \mathrm{sccm}$ and the undiluted $\mathrm{PH}_{3}$ was set at either 2 or $6 \mathrm{sccm}$. A summary of the five samples and the experimental conditions in which they were deposited is shown in Table 6.1. 


\begin{tabular}{ccccccc}
\hline ID & $\mathrm{B}_{2} \mathrm{H}_{6}$ & $\mathrm{PH}_{3}$ & $\mathrm{H}_{2}$ & $p$ (torr) & $T\left({ }^{\circ} \mathrm{C}\right)$ & $t(\min )$ \\
\hline L1 & 80 & 6 & 3500 & 100 & 1250 & 15 \\
L2 & 40 & 2 & 3500 & 100 & 1250 & 15 \\
L3 & 40 & 2 & 3500 & 100 & 1150 & 15 \\
L4 & 40 & 2 & 3500 & 100 & 1050 & 15 \\
L5 & 40 & 6 & 3500 & 100 & 1050 & 15 \\
\hline
\end{tabular}

TABlE 5.1: Experimental conditions during deposition of the five different samples studied in this work, with partial pressures of precursor gases in sccm, total pressure $p$ in torr, temperature $T$ in ${ }^{\circ} \mathrm{C}$ and deposition time $t$ in min.

\subsubsection{X-RAY ABSORPTION SPECTROSCOPY}

For each of the samples listed in Table 6.1, the x-ray absorption spectrum, at both the boron $K$-edge and the phosphorus $L_{2,3}$-edge, was collected in the total electron yield (TEY) mode, as shown in Fig. 6.1. The area under the curves has been normalized to unity to allow for meaningful comparison of relative changes in intensity of certain features. In this section we will focus the attention on the differences between the spectra collected for the different samples. Later in this article, we will provide analysis of the origin of these differences and the features themselves, using calculations from first principles.

For both edges, the spectra of samples deposited at a lower temperature are generally smoother than for samples deposited at higher temperatures; the latter have features that are more strongly defined. Typically, smoothened absorption features can be associated with distortions of the perfect crystalline lattice[1], due to thermally induced deformation of the crystalline network or localized amorphous phases. This is consistent with the notion that the likelihood of regions of the crystal growing amorphously, increases for decreasing deposition temperatures.

In the phosphorus $L_{2,3}$-edge spectrum, shown in Fig. 6.1 (a), we highlight four features $A, A^{\prime}, B$ and $C$ that are of particular interest. Unlike in the case of $K$-edge transitions, due to the energy splitting of the $2 p$ core level states by spin-orbit coupling, every unoccupied orbital that contributes to the overall absorption spectrum, will appear twice, separated by an energy of $\Delta E$, which in the case of phosphorus is approximately $0.85 \mathrm{eV}$. A good example of this can be seen at feature $A$ for sample L1, that has its corresponding spin-orbit coupled transition at feature $A^{\prime}$. There is a strong correlation between the decrease in intensity of feature $A$ and the decreasing deposition temperature of the sample which we will address in detail in the following sections, where we explain the origin of the feature and what structural defect causes its relative intensity to decrease. Features $B$ and $C$ are asymmetric in intensity for samples L1 and L2 but attain equal intensity for the lower temperature samples. 

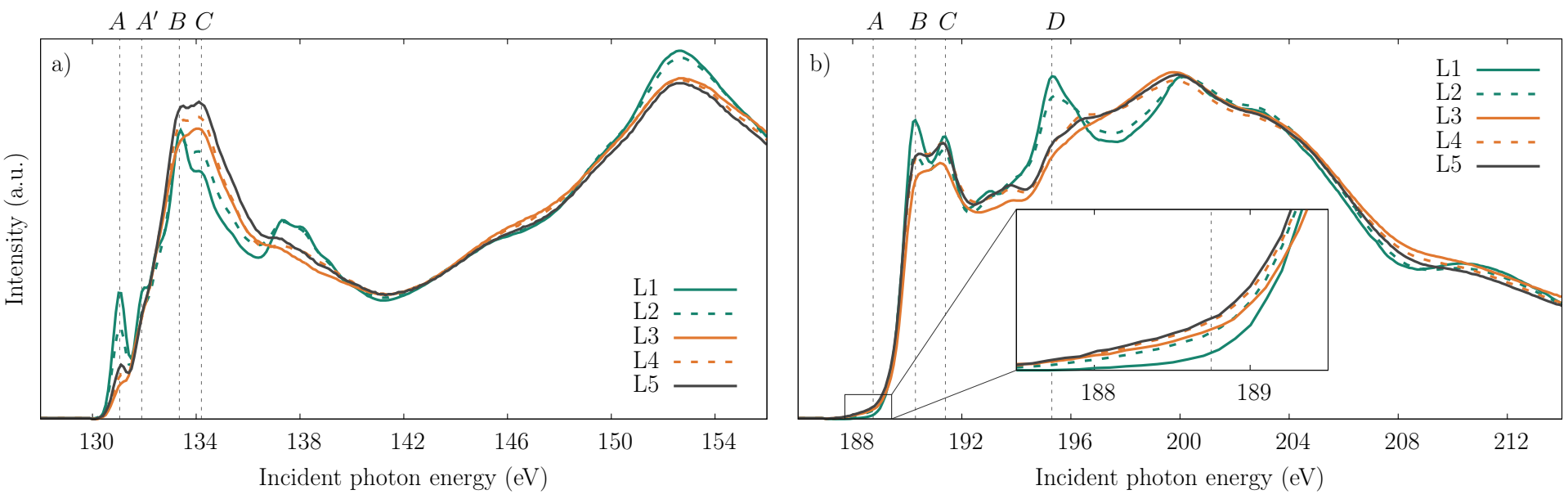

Figure 5.1: Experimentally collected x-ray absorption spectra for the (a) phosphorus $L_{2,3}$-edge and the (b) boron $K$-edge for five different $\mathrm{B}_{12} \mathrm{P}_{2}$ samples. The inset in panel (b) shows a close-up of the boron $K$-edge absorption pre-edge feature. The relative intensity is increasing for a decreasing deposition temperature of the sample. 
Turning our attention to the boron $K$-edge spectrum in Fig. 6.1(b), we observe the same trend as for the phosphorus $L_{2,3}$-edge, where features become less well defined for samples with a lower deposition temperature. Features $B$ and $C$ become less well defined for samples with lower deposition temperatures and their intensity ratio changes. Samples L1 and L2 show a relatively strong transition at feature $D$ which becomes suppressed for the other samples. All samples also showed a weak pre-edge transition, denoted as feature $A$, which is stronger with respect to the rest of the spectral features as the deposition temperature decreases. Similar to feature $A$ in the phosphorus $L_{2,3^{-}}$ edge, this feature can be related to a structural defect which will be discussed in detail in later sections.

\subsection{COMPUTATIONAL RESULTS}

\subsubsection{CRYSTAL STRUCTURE}

A schematic representation of the crystal structure of $\mathrm{B}_{12} \mathrm{P}_{2}$ is shown Fig. 5.2. The fundamental building block of $\mathrm{B}_{12} \mathrm{P}_{2}$ is the boron icosahedron, which is a structural unit of 12 boron atoms that occupy the vertices of a regular icosahedron. These boron icosahedra form an orthorhombic lattice that are interconnected with dimers of phosphorus atoms, oriented parallel to the longest diagonal of the rhombohedral cell. The crystal structure has only three symmetrically unique crystal lattice sites, a phosphorus site, a boron site bound to a phosphorus atom and a boron site bound to a boron atom from a neighboring icosahedron. These different boron sites will be referred to as P-bound and B-bound boron sites and are shown in Fig. 5.2 (b) as black and light gray spheres, respectively.

\subsubsection{FORMATION ENERGIES OF POINT DEFECTS}

The three unique lattice sites in the $\mathrm{B}_{12} \mathrm{P}_{2}$ crystal structure, give a total of 6 symmetrically inequivalent antisite and vacancy defects, a boron antisite $\mathrm{B}_{\mathrm{P}}$, a B-bound phoshporus antisite $\mathrm{P}_{\mathrm{B}(\mathrm{B})}$, a $\mathrm{P}$-bound phosphorus antisite $\mathrm{P}_{\mathrm{B}(\mathrm{P})}$, a B-bound boron vacancy $\mathrm{V}_{\mathrm{B}(\mathrm{B})}$, a $\mathrm{P}$-bound vacancy $\mathrm{V}_{\mathrm{B}(\mathrm{P})}$ and a phosphorus vacancy $\mathrm{V}_{\mathrm{P}}$. In addition, we consider interstitial boron defects, however in stark contrast with antisite and vacancy defects, the position of interstitial defects is by definition not known a priori. In this work, we will use the structural definition and naming convention of two interstitial boron defects $\mathrm{I}_{\mathrm{B}}^{\mathrm{A}}$ and $\mathrm{I}_{\mathrm{B}}^{\mathrm{B}}$, as described in another publication[84]. We have created each one of these defects in a $2 \times 2 \times 2 \mathrm{~B}_{12} \mathrm{P}_{2}$ supercell and computed the formation energies of the relaxed defects in two limits, that represent a boron-rich and phosphorus-rich environment, as shown in Table 5.2. 

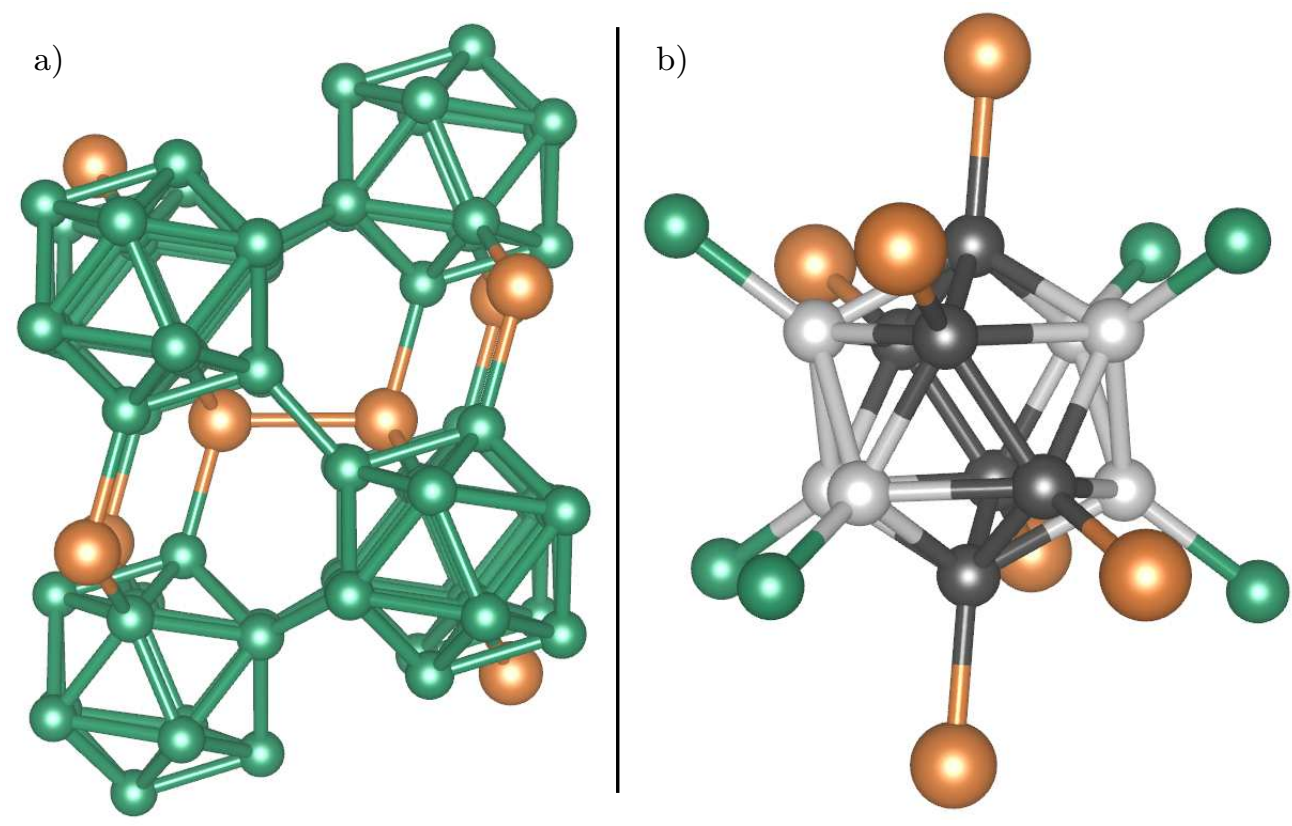

Figure 5.2: (a) A schematic depiction of the crystal structure of $\mathrm{B}_{12} \mathrm{P}_{2}$, where orange (light) spheres represent phosphorus atoms and green (darker) spheres represent boron atoms. This depiction shows six complete boron icosahedra in an orthorhombic formation with one complete phosphorus dimer positioned in the center. Panel (b) depicts a single complete icosahedron with twelve external $\mathrm{B}$ and $\mathrm{P}$ atoms it is bound to. Boron atoms bound to an external boron atom (B-bound) are indicated by a light grey sphere, whereas boron atoms bound to an external phosphorus atom (P-bound) are indicated by black spheres. Boronboron, boron-phosphorus and phosphorus-phosphorus bonds are drawn up to a maximum of 2,2 and $3 \AA$, respectively. 


\begin{tabular}{lcc}
\hline Defect & Boron-rich & Phosphorus-rich \\
\hline $\mathrm{B}_{\mathrm{P}}$ & 2.05 & 4.52 \\
$\mathrm{P}_{\mathrm{B}(\mathrm{B})}$ & 5.89 & 3.42 \\
$\mathrm{P}_{\mathrm{B}(\mathrm{P})}$ & 7.16 & 4.69 \\
$\mathrm{~V}_{\mathrm{B}(\mathrm{B})}$ & 5.02 & 4.66 \\
$\mathrm{~V}_{\mathrm{B}(\mathrm{P})}$ & 5.45 & 5.10 \\
$\mathrm{~V}_{\mathrm{P}}^{\mathrm{A}}$ & 4.17 & 6.29 \\
$\mathrm{I}_{\mathrm{B}}^{\mathrm{A}}$ & 4.84 & 5.20 \\
$\mathrm{I}_{\mathrm{B}}^{\mathrm{B}}$ & 5.06 & 5.41 \\
\hline
\end{tabular}

TABLE 5.2: Formation energies in eV, of boron and phosphorus, vacancies and antisites, as well as boron interstitial defects, under boron-rich and phosphorus-rich conditions. The chemical potentials for boron and phosphorus atoms were referenced with respect to the formation enthalpy of pure icosahedral boron and orthorhombic phosphorus solids.

All defect structures relaxed with only minor deformations of the defective icosahedron or any of the directly neighboring icosahedra, which shows that a boron icoshedron maintains structural integrity even when in a degraded state in the presence of vacancy or interstitial defect. However, the formation energies for all of the considered defects are significant ranging between $2 \mathrm{eV}$ to $7 \mathrm{eV}$, approximately. Under boron-rich conditions, the computed formation energies indicate that boron antisites and phosphorus vacancies are the most likely defects, of those considered, to be formed. When the conditions are phosphorus-rich, not surprisingly, the phosphorus vacancy has a larger formation energy, however, the boron antisite remains relatively favorable. The most likely point defect, under phosphorus rich conditions, based on these formation energies, is the phosphorus antisite at a B-bound boron crystal site. It is interesting to note that boron vacancies have relatively large formation energies in both the limits of boron- and phosphorus-richness.

\subsubsection{DENSITY OF STATES}

The density of states of the $\mathrm{B}_{12} \mathrm{P}_{2}$ crystal was calculated for both a generalized gradient approximation (PBE) and a hybrid (HSE06) exchange-correlation functional. The calculation using the PBE functional finds an indirect band gap of $2.42 \mathrm{eV}$, which compares well to other theoretical values 2.5[88], 2.63[89] and $2.79 \mathrm{eV}[90]$, reported in literature. The generalized gradient approximation is infamous for underestimating the band gap, and compared to the experimental value of $3.35 \mathrm{eV}[91]$, reported for $\mathrm{B}_{12} \mathrm{P}_{2}$, this is no exception. Hybrid functionals, which include a portion of exact exchange from Hartree-Fock theory in the exchange-correlation functional, are often used successfully to correct this underestimation. The density of states computed using the HSE06 functional, as shown in Fig. 5.3, shows a band gap of $3.39 \mathrm{eV}$, which agrees well with 


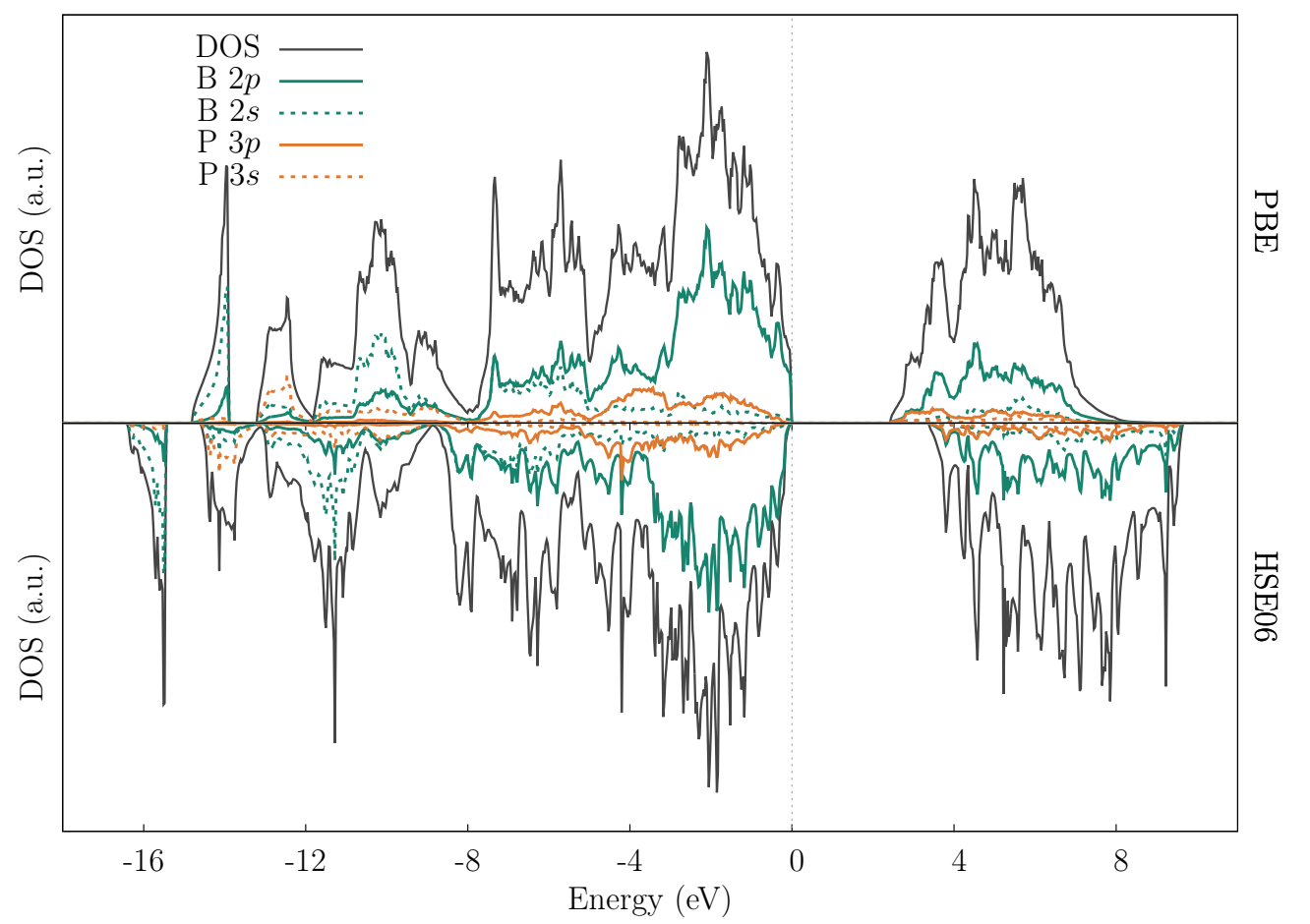

Figure 5.3: Total density of states (black solid line) and partial density of states for B $2 p$ (solid green), B $2 s$ (dashed green), P $2 p$ (solid orange) and $\mathrm{P} 2 s$ (dashed orange) orbitals computed for a PBE and HSE06 exchange-correlation functional. The energy is aligned with respect to the Fermi level, indicated by the vertical dashed line.

experiment. Apart from the distinctive difference in energy scales and the resulting difference in the band gap, the density of states for the two different functionals agree well. The top of the valence band is dominated by states that are located on the boron atoms of the icosahedra and have a distinctive $p$-like character and there is almost no contribution from states on the phosphorus atoms. Contrastingly, the bottom of the conduction band comprises both B $2 p$ and P $3 p$ states.

\subsubsection{SIMULATED X-RAY ABSORPTION SPECTROSCOPY}

In order to understand and further analyze the experimentally collected x-ray absorption spectra, we have simulated the phosphorus $L_{2,3}$-edge and boron $K$-edge for the perfect $\mathrm{B}_{12} \mathrm{P}_{2}$ crystal structure with the DFT-XCH method. The results are shown in Fig. 5.4. 

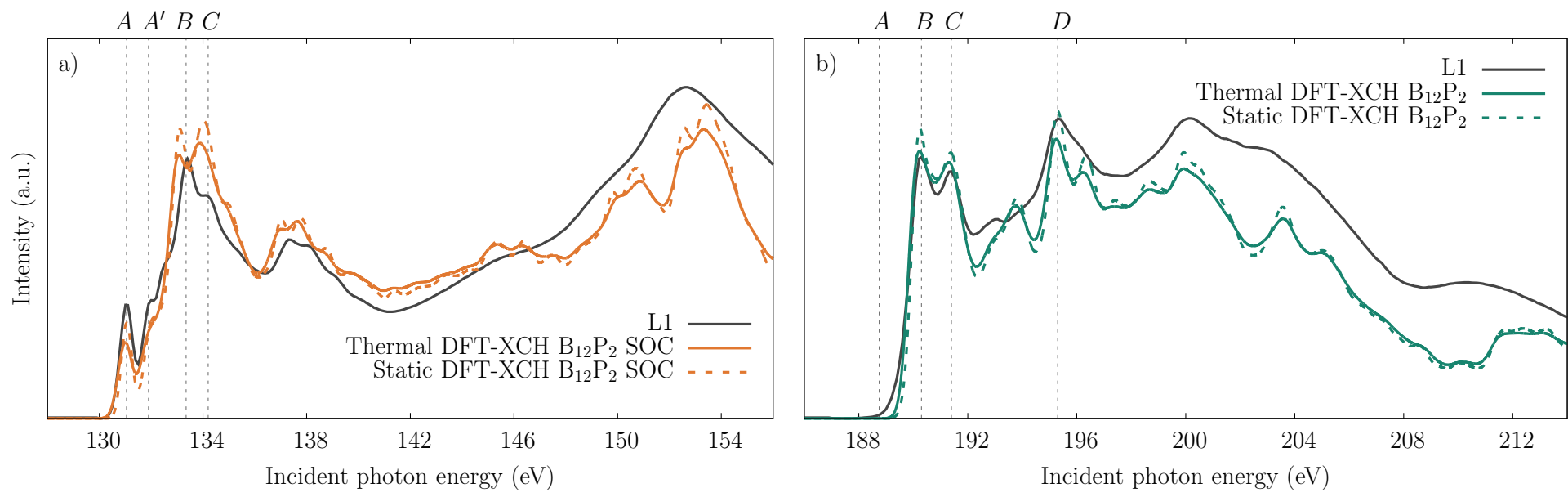

Figure 5.4: Simulated x-ray absorption spectra of the (a) phosphorus $L_{2,3}$-edge and (b) boron $K$-edge for the perfect $\mathrm{B}_{12} \mathrm{P}_{2}$ crystal structure at $0 \mathrm{~K}$ (colored dashed) and at $300 \mathrm{~K}$ (colored solid). For comparison, the experimental spectrum for sample L1 is plotted in both panels with a solid black line. The feature labels are identical to those in Fig. 6.1. 

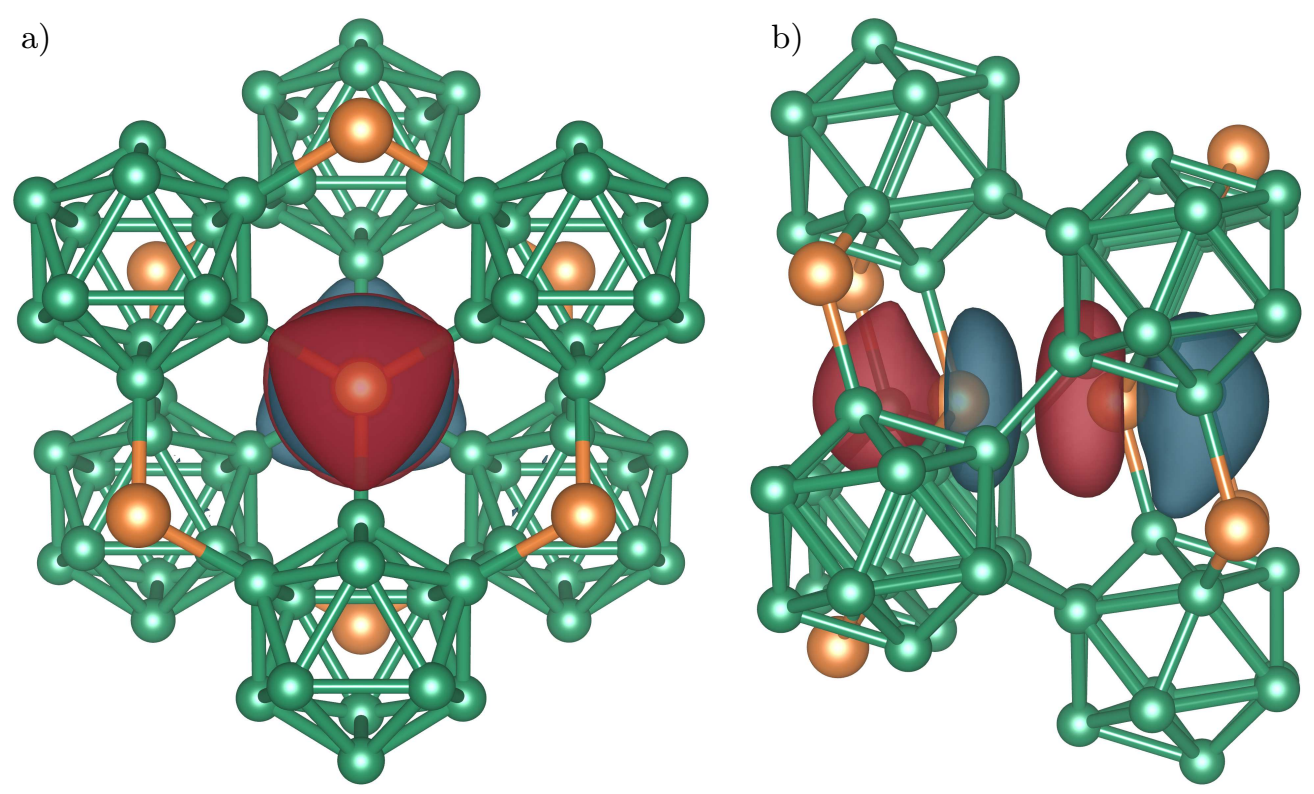

Figure 5.5: Visual representation of an isosurface of the squared wavefunction $|\psi(\vec{r})|^{2}$ of the state corresponding to the intense transition $A$ at $131 \mathrm{eV}$ in the $\mathrm{P} L_{2,3}$-edge spectrum, as seen along the (a) $\langle 00\rangle$ direction and (b) $\langle 010\rangle$ of the crystal. The excitonic state is highly localized on the phosphorus bond and has distinct $\sigma^{*}$ character. The two different colors of the isosurface represent the different phase of the wavefunction.

The colored dashed lines in Fig. 5.4 represent the spectrum for the static crystal at a temperature of $0 \mathrm{~K}$ and the solid colored lines represent a statistical average of structural snapshots sampled from a trajectory of the perfect crystal equilibrated at $300 \mathrm{~K}$. Overall the simulated spectra agree well with experiment, both for the static and thermal case. Usually, thermal distortions induced by finite temperature will smoothen the high frequency oscillations in the spectrum, as the summation of spectra from individual atoms, while they are slightly displaced from their equilibrium crystalline positions, from multiple snapshots, replicates the statistical averaging over time of the experiment[1]. In the case of $\mathrm{B}_{12} \mathrm{P}_{2}$ the effect of thermal structural distortions is minimal, with just a slight red shift of the onset of absorption for the boron $K$-edge.

The isolated pre-edge feature $A$ in the $\mathrm{P} L_{2,3}$-edge spectrum, mirrored in feature $A^{\prime}$ through spin-orbit coupling by transitions from the deeper $p_{1 / 2}$ core states into the same unoccupied orbital, can be attributed to an excited state with a strong $\sigma^{*}$ character that is highly localized on the phosphorus dimer bond of the excited phosphorus atom, as visualized in Fig. 5.5. Feature $C$ can be attributed to a state with negative electronic density on the phosphorus dimer, with a ring of positive electronic density concentric with the excited phosphorus atom, as displayed in Fig. 5.6. The outer ring of electronic density connects the three directly neighboring icosahedra that are bound to the excited 

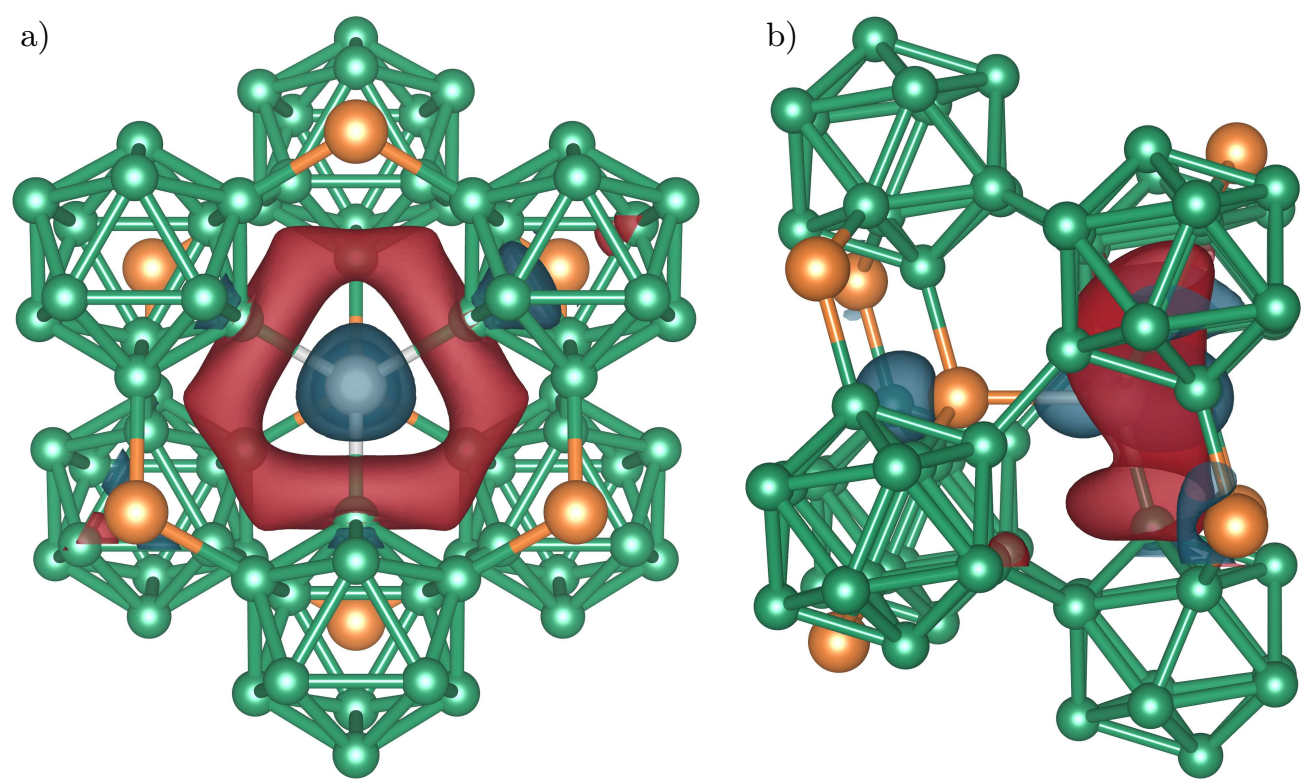

Figure 5.6: Visual representation of an isosurface of the squared wavefunction $|\psi(\vec{r})|^{2}$ of the state corresponding to the intense transition $C$ at $134 \mathrm{eV}$ in the $\mathrm{P} L_{2,3}$-edge spectrum, as seen along the (a) $\langle 00\rangle$ direction and (b) $\langle 010\rangle$ of the crystal. The two different colors of the isosurface represent the different phase of the wavefunction.

phosphorus atom. The intensity of feature $B$ originates from transitions into excited states at $k$-points other than the $\Gamma$ point and therefore a meaningful visualization can not be made.

In the case of the B $K$-edge, there are two populations of symmetrically inequivalent boron atoms, B-bound and P-bound, that each have a distinct absorption spectrum. Fig. 5.4 shows the average of these two spectra but a deconvoluted spectrum for Bbound and P-bound boron atoms can be found Figs. 5.7 (a) and 5.7 (b), respectively. B-bound atoms predominantly contribute to features $B, C$ and $D$ in Fig. 5.4 (b). The state that gives rise to feature $B$ is highly localized on the icosahedron containing the excited boron atom and consists of 12 lobes with distinct antibonding character. The excited state attributable to feature $C$, is slightly more delocalized compared to the previously described state, but remains mainly localized on the excited icosahedron. Directly on the excited boron atom, lobes with $p$-character are found, but the electronic density also extends along the bonds that the icosahedron forms with neighboring external atoms. Finally, the excited state giving rise to feature $D$ has mostly $\pi^{*}$-like character located on the surface of the icosahedron and along the bonds it forms with its neighboring phosphorus atoms. The excited states, corresponding to the described features $B, C$ and $D$, as just described, are visualized in Figs. 5.8, 5.9 and 5.10, with the corresponding labels $S_{1}, S_{2}$ and $S_{3}$ in Fig. 5.7 (a), respectively. 

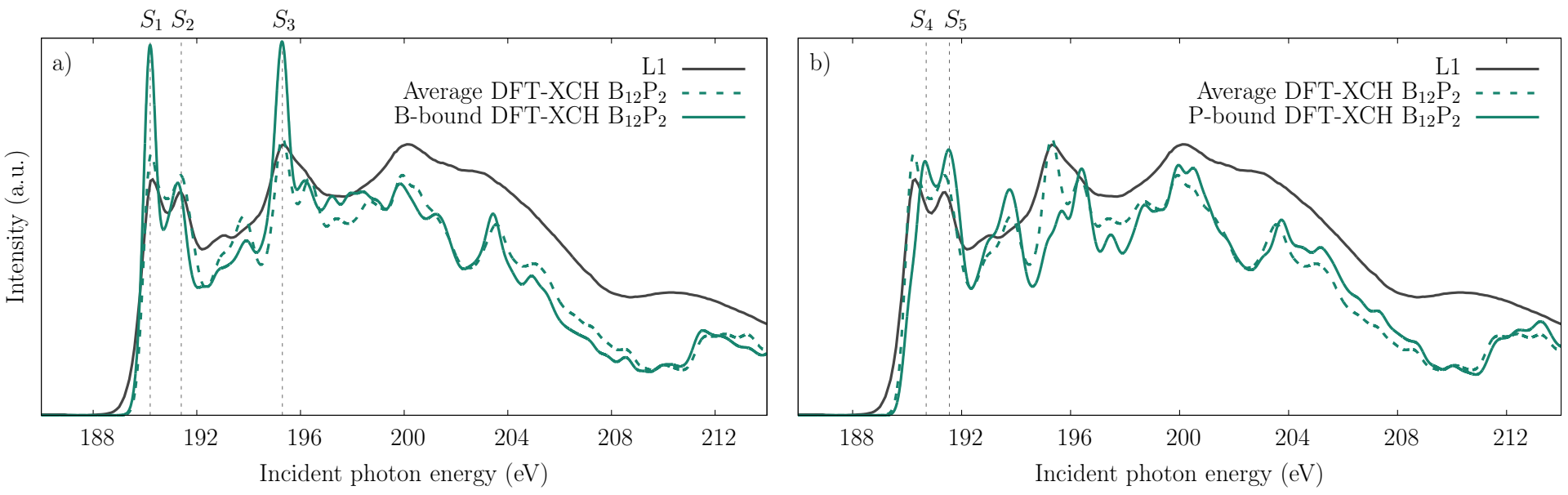

Figure 5.7: Deconvoluted average computed B $K$-edge for the static $\mathrm{B}_{12} \mathrm{P}_{2}$ crystal cell for (a) B-bound and (b) P-bound boron atoms. The solid green line represents the respective partial spectrum and the dashed green line is the average of the B-bound and P-bound spectrum, which corresponds directly to the dashed green line shown in Fig. 5.4 (b). The solid black line corresponds to the experimental spectrum collected for sample L1. The vertical dashed lines indicate the positions of states that are most intense and dominate the shape of the partial spectra. Visual representations of the electronic density of these states, are displayed in Figs. 5.8 to 5.12, corresponding to the labels $S_{1}$ to $S_{5}$, respectively. 


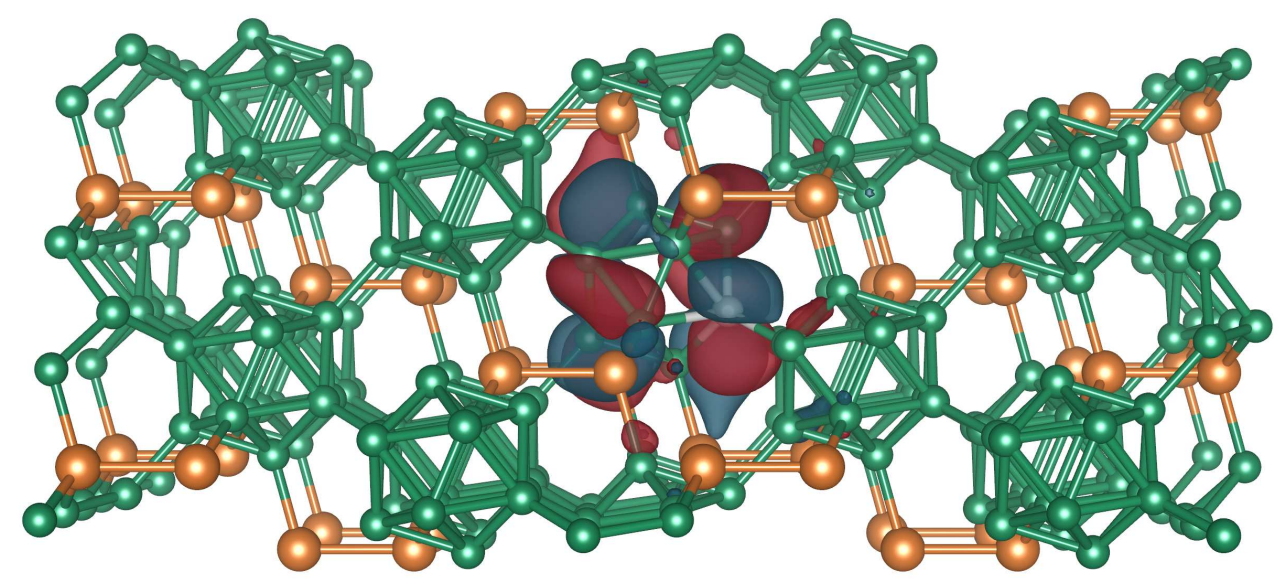

Figure 5.8: Visual representation of an isosurface of the squared wavefunction $|\psi(\vec{r})|^{2}$ of the state corresponding to the intense transition $S_{1}$ in the B $K$-edge spectrum of B-bound atoms [see Fig. $5.7(\mathrm{a})$ ], as seen along the $\langle 010\rangle$ of the crystal. The excited boron atom is shown in gray and the two different colors of the isosurface represent the different phase of the wavefunction.

The onset of absorption from P-bound boron atoms is slightly blue shifted compared to the transitions from B-bound atoms due to the discrepancy in local electron density which is driven by the difference in electronegativity between boron and phosphorus, as can be seen in the comparison between Figs. 5.7 (b) and 5.7 (a). A boron atom bound to a phosphorus atom will effectively give up more of its electrons therefore reducing the valence screening of core excitations and increasing their binding energy. The minimum required energy to excite a core electron into the first unoccupied orbital is therefore increased, which explains the blue shift. Excitations of core electrons from P-bound boron atoms mostly contribute to features $B$ and $C$, predominantly due to excitation of the core electron into two particular states. These states, labeled $S_{4}$ and $S_{5}$ in Fig. 5.7 (b) and visualized in Figs. 5.11 and 5.12, have similar character and localization compared to the states $S_{1}$ and $S_{2}$, respectively, which are the dominant states in the absorption spectrum of B-bound atoms. Feature $A$ is clearly not reproduced by the simulation for the perfect crystal, which suggests that states that contribute to this part of the spectrum may be associated with states that are associated with structural defects of the crystal. We will discuss this in more detail in the following sections. 


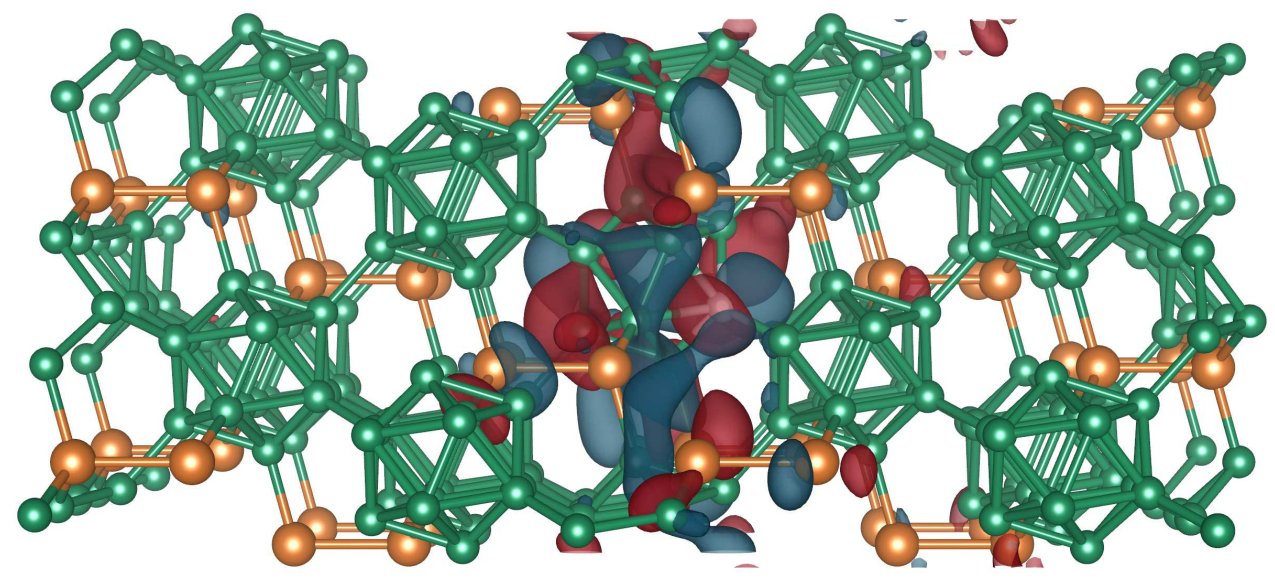

Figure 5.9: Visual representation of an isosurface of the squared wavefunction $|\psi(\vec{r})|^{2}$ of the state corresponding to the intense transition $S_{2}$ in the B $K$-edge spectrum of B-bound atoms [see Fig. $5.7(\mathrm{a})$ ], as seen along the $\langle 010\rangle$ of the crystal. The excited boron atom is shown in gray and the two different colors of the isosurface represent the different phase of the wavefunction.

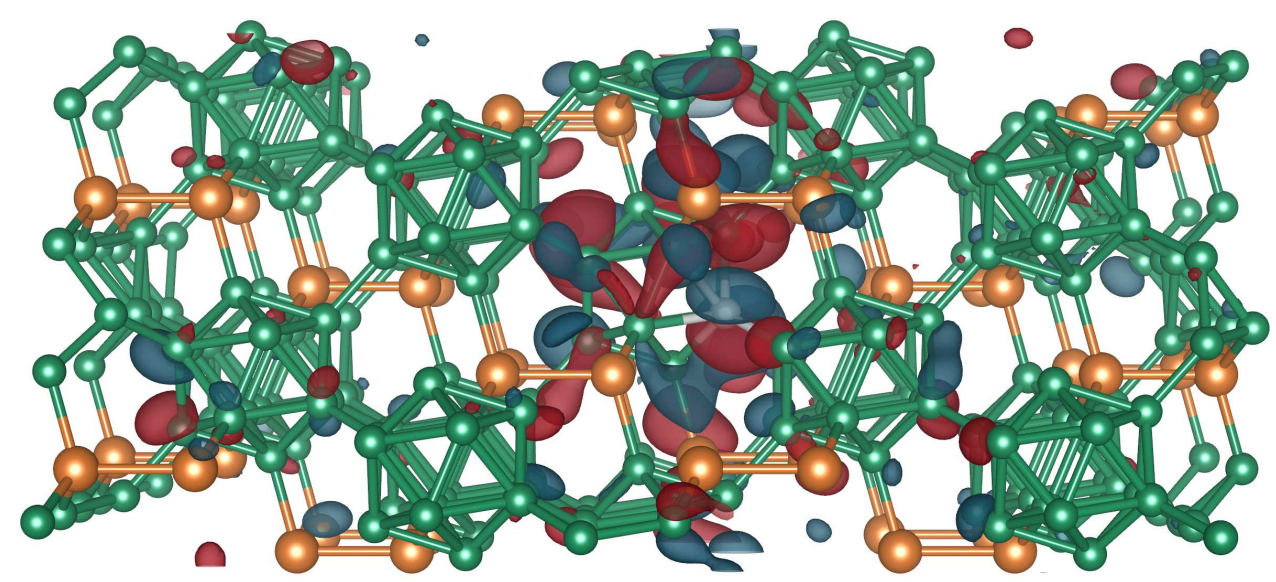

FiguRE 5.10: Visual representation of an isosurface of the squared wavefunction $|\psi(\vec{r})|^{2}$ of the state corresponding to the intense transition $S_{3}$ in the B $K$-edge spectrum of B-bound atoms [see Fig. 5.7 (a)], as seen along the $\langle 010\rangle$ of the crystal. The excited boron atom is shown in gray and the two different colors of the isosurface represent the different phase of the wavefunction. 


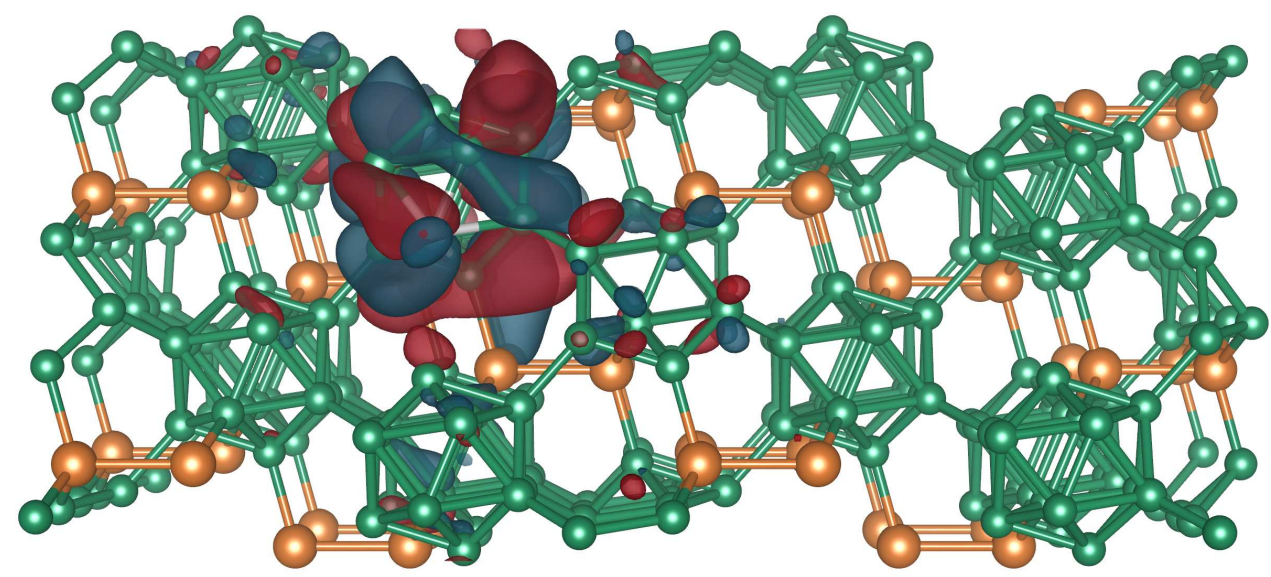

Figure 5.11: Visual representation of an isosurface of the squared wavefunction $|\psi(\vec{r})|^{2}$ of the state corresponding to the intense transition $S_{4}$ in the B $K$-edge spectrum of P-bound atoms [see Fig. 5.7 (b)], as seen along the $\langle 010\rangle$ of the crystal. The excited boron atom is shown in gray and the two different colors of the isosurface represent the different phase of the wavefunction.

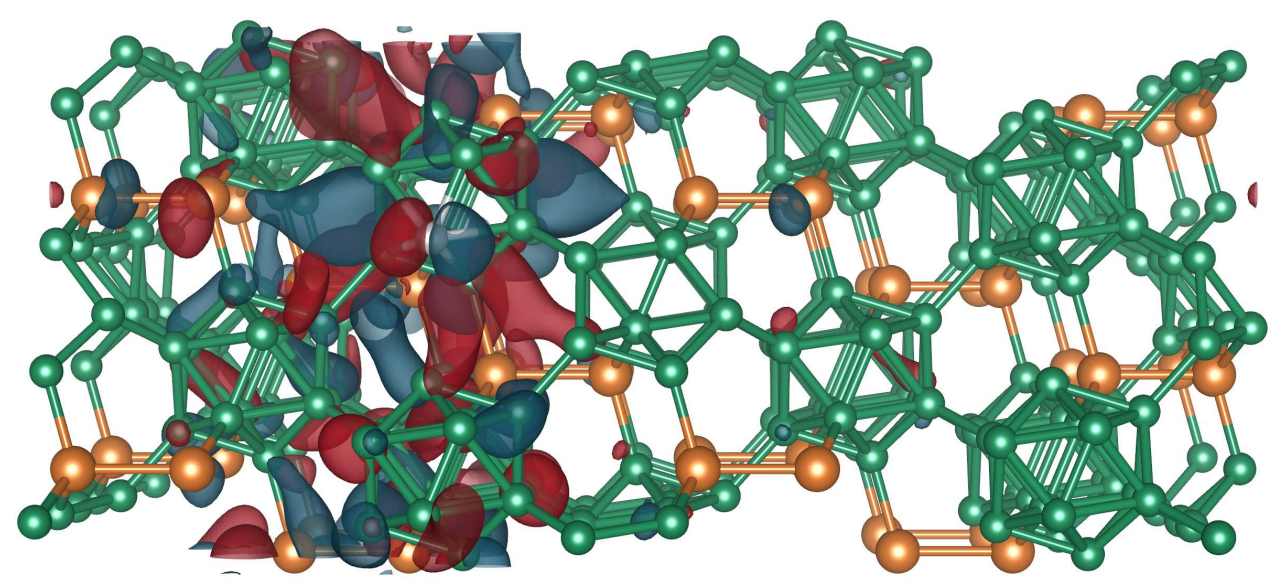

Figure 5.12: Visual representation of an isosurface of the squared wavefunction $|\psi(\vec{r})|^{2}$ of the state corresponding to the intense transition $S_{5}$ in the B $K$-edge spectrum of P-bound atoms [see Fig. 5.7 (b)], as seen along the $\langle 010\rangle$ of the crystal. The excited boron atom is shown in gray and the two different colors of the isosurface represent the different phase of the wavefunction. 


\subsubsection{DEFECT STATES AND SPECTRAL ALIGNMENT}

X-ray absorption spectra, as computed within the DFT-XCH approach, have relative energy scales and therefore, in order to allow for meaningful comparison of spectra of different systems, an energy alignment procedure, with respect to an objective reference, is required. In this work, we have adopted the approach of England et al.[67], which aligns the spectra with respect to the excitation energy of an isolated atom of the element of interest and the Kohn-Sham eigenvalue of the lowest energy unoccupied state into which the core-excited electron is placed. However, both the relative excitation energy, as computed from DFT total energies, and the Kohn-Sham eigenvalues include fictitious self-interaction effects. These fictitious contributions arise from the interaction of electronic orbitals with themselves as part of the effective potential of the system and become more significant for increasingly localized electronic states. Point defects often introduce defect states in the band gap of a material, which typically have a highly localized character. The point defects in $\mathrm{B}_{12} \mathrm{P}_{2}$ considered in this work are no exception and to illustrate this, the ground state density of states for the defects $\mathrm{P}_{\mathrm{B}(\mathrm{P})}$ and $\mathrm{V}_{\mathrm{P}}$ has been plotted in Fig. 5.13.

Both defects clearly introduce one or multiple defect states that are located in the band gap. In the case of $\mathrm{P}_{\mathrm{B}(\mathrm{P})}$, the defect state is completely occuppied (determined by the Fermi level indicated by the dashed line), in contrast with the phosphorus vacancy $\mathrm{V}_{\mathrm{P}}$, which introduces multiple defect states, several of which (indicated by an arrow in Fig. 5.13) remain unoccupied in the ground state. When the simulated system is core-excited, the electron is placed in the first available empty state, whose Kohn-Sham eigenvalue will be used in the energy alignment scheme, including the self-interaction contribution contained within it. As long as all systems that are compared with one another, have a first available empty state with similar character and localization, the self-interaction error will be comparable and will cancel out in the relative alignment. In this situation, this is the case for $\mathrm{P}_{\mathrm{B}(\mathrm{P})}$, for which the first available empty state is the bottom of the conduction band and therefore directly comparable to the case of the pristine crystal, however, in the case of the $V_{P}$ defect, the state into which the core-electron will be excited, is a defect state, which has distinct different character. As a result, the self-interaction error will also be different and the relative alignment scheme may fail.

To illustrate this, the highest occupied molecular orbital (HOMO), for the excited state of a $\mathrm{B}_{12} \mathrm{P}_{2}$ crystal containing either a $\mathrm{P}_{\mathrm{B}(\mathrm{P})}$ or $\mathrm{V}_{\mathrm{P}}$ defect and a reference atom far away from the structural defect in its core-excited state, is visualized in Figs. 5.14 (a) and 5.14 (b), respectively. The isosurface values were chosen such that an equal amount of the total charge density is contained within the respective surfaces and the isosurface representation of the HOMO in the bulk system shown in Fig. 5.5. This enables a direct comparison of the locality of the excited states. The HOMO of the core-hole excited system containing the $\mathrm{P}_{\mathrm{B}(\mathrm{P})}$ defect has a strong $\sigma^{*}$ character and strongly 


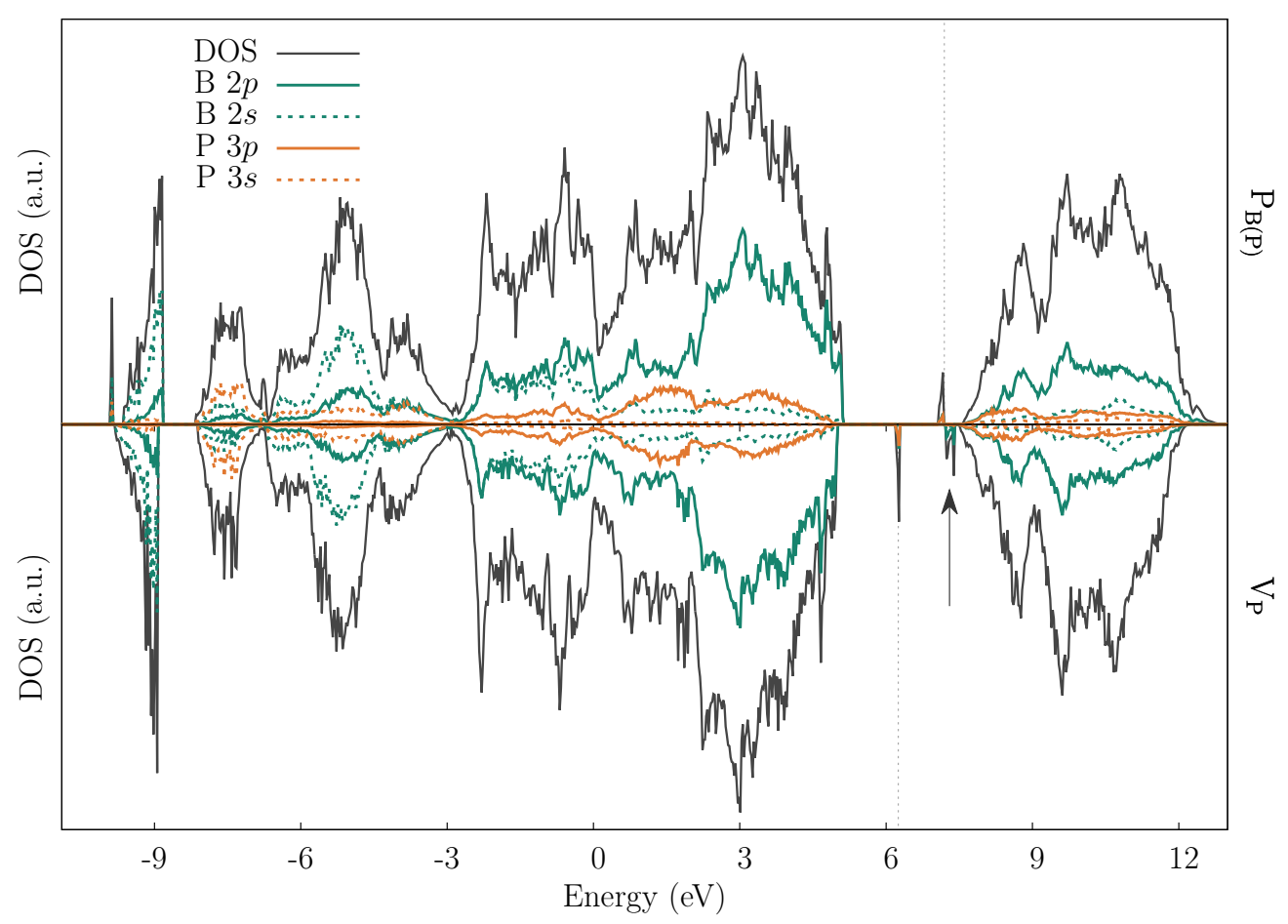

Figure 5.13: Ground state total density of states (black solid line) and partial density of states for B $2 p$ (solid green), B $2 s$ (dashed green), $\mathrm{P} 2 p$ (solid orange) and $\mathrm{P} 2 s$ (dashed orange) orbitals computed for the $\mathrm{B}_{12} \mathrm{P}_{2}$ supercell containing a (a) $\mathrm{P}_{\mathrm{B}(\mathrm{P})}$ or (b) $\mathrm{V}_{\mathrm{P}}$ defect. The Fermi level is indicated by a vertical dashed line. Defect states that are unoccupied in the ground state for the $V_{P}$ defect, are indicated by the black arrow. 

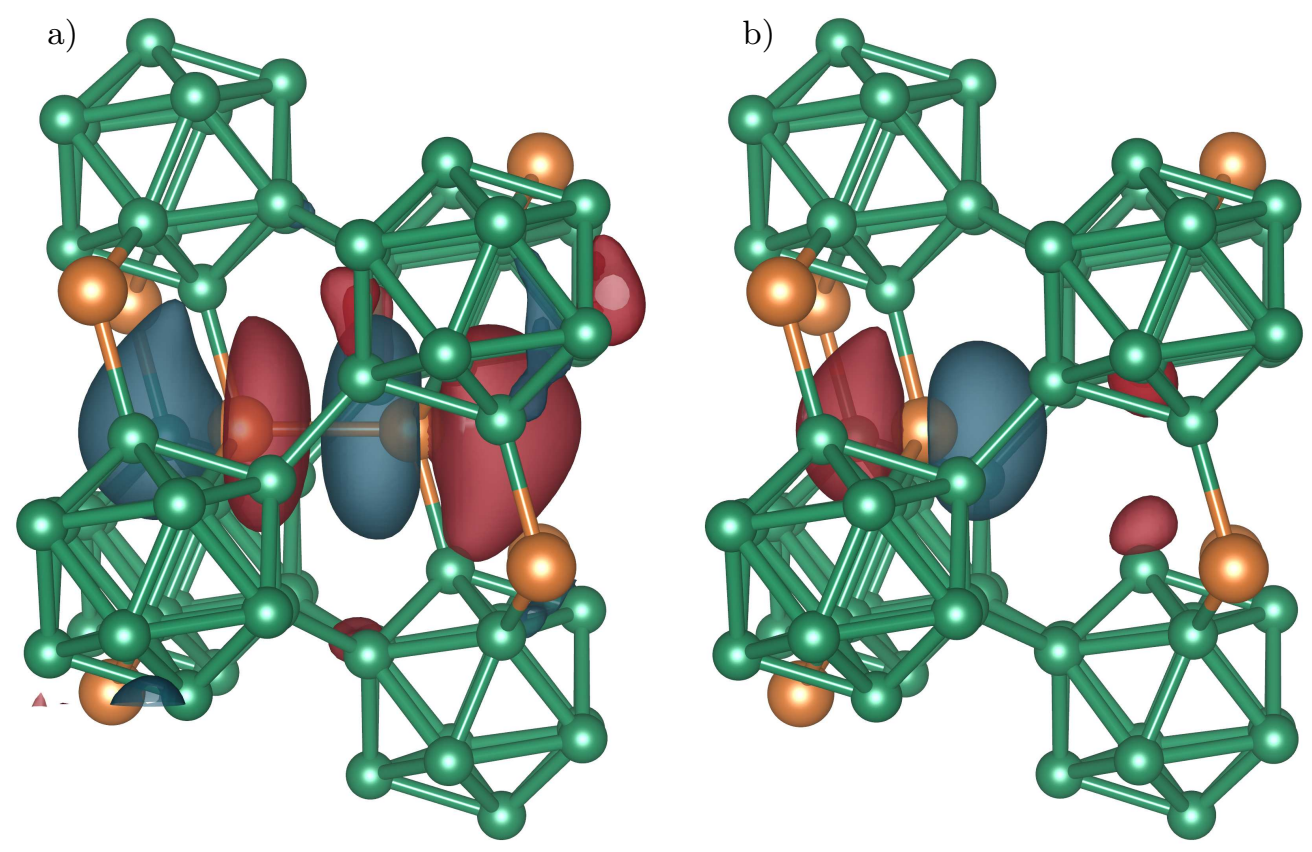

FIGURE 5.14: Visual representation of an isosurface of the squared wavefunction $|\psi(\vec{r})|^{2}$ of the highest occupied electronic states in the excited state of a system containing a (a) $\mathrm{P}_{\mathrm{B}(\mathrm{P})}$ or (b) $\mathrm{V}_{\mathrm{P}}$ defect. The core-hole excited atom is approximately $10 \AA$ removed from the structural defect and the excited state is localized on the excited boron atom in the case of the $\mathrm{P}_{\mathrm{B}(\mathrm{P})}$ defect. This is however not necessarily the case, as clearly, for the $V_{P}$ defect, the excited state is not localized on the excited atom, but rather on the $V_{P}$ defect itself. 


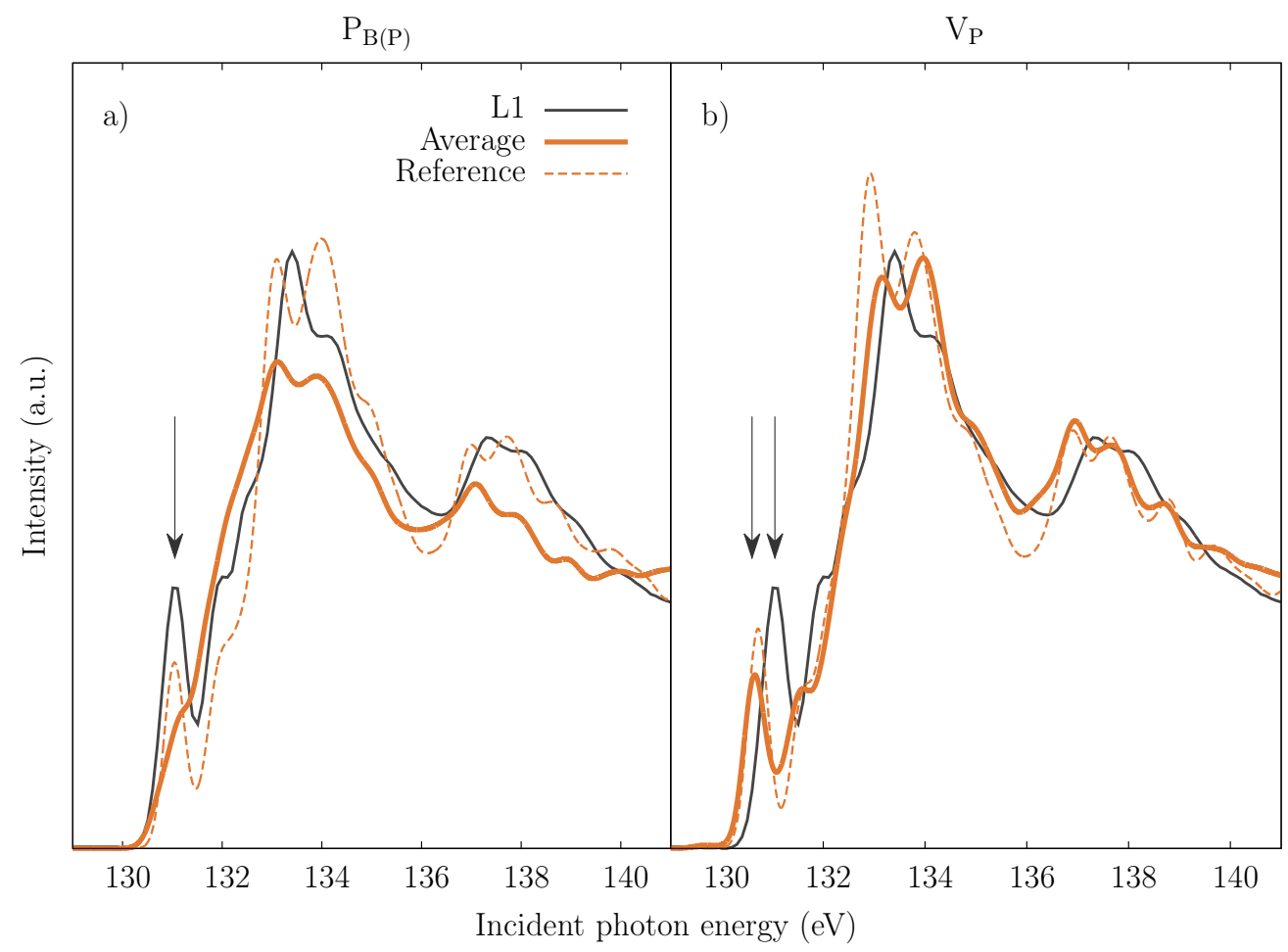

Figure 5.15: Experimental P $L_{2,3}$ spectrum of sample L1 (solid black line) and the computed spectra (orange lines) for a $\mathrm{B}_{12} \mathrm{P}_{2}$ supercell containing a $\mathrm{P}_{\mathrm{B}(\mathrm{P})}$ or $\mathrm{V}_{\mathrm{P}}$ defect, in the left and right panel, respectively. The solid orange lines represent the average spectrum of all the atoms in the direct vicinity of the defect, whereas the dashed orange line is the computed spectrum of a reference atom far removed from the structural defect. The vertical black arrows indicate the locations of the $\sigma^{*}$ transitions for both the experimental and simulated spectra.

resembles the HOMO of the core-excited perfect crystal [see Fig. 5.5 (b)] and both states are comparably localized, as predicted by the calculated density of states. However, contrastingly, the HOMO for the $\mathrm{V}_{\mathrm{P}}$ containing system, which corresponds to the empty state in the ground state density of states marked by the arrow in Fig. 5.13 (b), clearly has a more localized character, in this case due to the missing phosphorus atom. Due to the increased locality of this HOMO compared to the HOMO in the bulk system, the self-interaction energy error will increase and when using the same absolute alignment energy for both systems, the spectrum of the defective system will be red-shifted compared to experiment.

This can be seen in Figs. 5.15 (a) and 5.15 (b), which shows the computed x-ray absorption spectra for the $\mathrm{P}_{\mathrm{B}(\mathrm{P})}$ and $\mathrm{V}_{\mathrm{P}}$ containing system. The black solid line represents the measured spectrum for sample L1, whereas the solid orange line corresponds to the computed average spectrum for all atoms within a radius of $3.3 \AA$ of the structural 
defect and the dashed orange line is the computed spectrum for the distant reference atom, whose first excited state was visualized in Fig. 5.14(b). For the $\mathrm{P}_{\mathrm{B}(\mathrm{P})}$ defect, the $\sigma^{*}$ feature aligns perfectly with experiment, as the self-interaction error is comparable to that of the bulk calculation for which the initial alignment was done, however for the phosphorus vacancy, there is a significant mismatch of almost $0.5 \mathrm{eV}$. Note that the mismatch is also present for the spectrum of the reference atom, far removed from the defect. In this particular case, the mismatch for the average and reference atom spectrum are identical, but this is not necessarily always the case and therefore correcting the self-interaction alignment error by aligning with respect to the reference atom, is not an option. A robust scheme to correct for the self-interaction error in the current energy alignment scheme is not available. In this work, this problem is overcome by directly aligning the individual atomic spectra of a specific structural configuration with respect to the position of the $\sigma^{*}$ feature, relative to the same feature in the computed spectrum for the bulk material.

\subsubsection{POINT DEFECT X-RAY ABSORPTION SPECTROSCOPY}

To investigate the influence of structural point defects of the crystalline structure on the $\mathrm{x}$-ray absorption spectrum of $\mathrm{B}_{12} \mathrm{P}_{2}$, and whether they can explain the differences in the experimental spectra and reproduce features that are not predicted for the perfect crystal lattice, we consider vacancy, interstitial and antisite defects. We have computed the average x-ray absorption spectrum of boron and phosphorus atoms that are within a radius of 3.4 and $4.0 \AA$, respectively, of the point defect. Either the appearance of new features or the reduction of features that were present in the crystalline spectrum, can provide evidence for which point defects are present in the experimental samples. These changes are most apparent at the onset of the absorption spectrum and therefore Fig. 5.16 shows the onset of the computed x-ray absorption spectra for the eight different point defects listed in Table 5.2, for both the boron $K$-edge and phosphorus $L_{2,3}$-edge.

A perfect example of defects that introduce new spectral features, are boron vacancies, which open defect states within the band gap and as boron atoms, that are directly adjacent to the vacancy, are core excited into these defect states, a strong absorption feature appears below the main absorption edge of the B $K$-edge spectrum. For both types of boron vacancies, this defect feature is predicted to appear at an energy of $187 \mathrm{eV}$, which is far below the energy of the main absorption edge found in experiment. Even though this result suggests a lack of boron vacancies in the experimental samples, this does not guarantee a complete absence of boron vacancies, as the chance of detecting the defect feature depends on both the intensity resolution of the experimental setup, as well as the oscillator strength of the specific defect state and the relative abundance of vacancies present in the sample. Our simulations predict an oscillator strength for boron vacancy defect states that is approximately half the intensity of the 


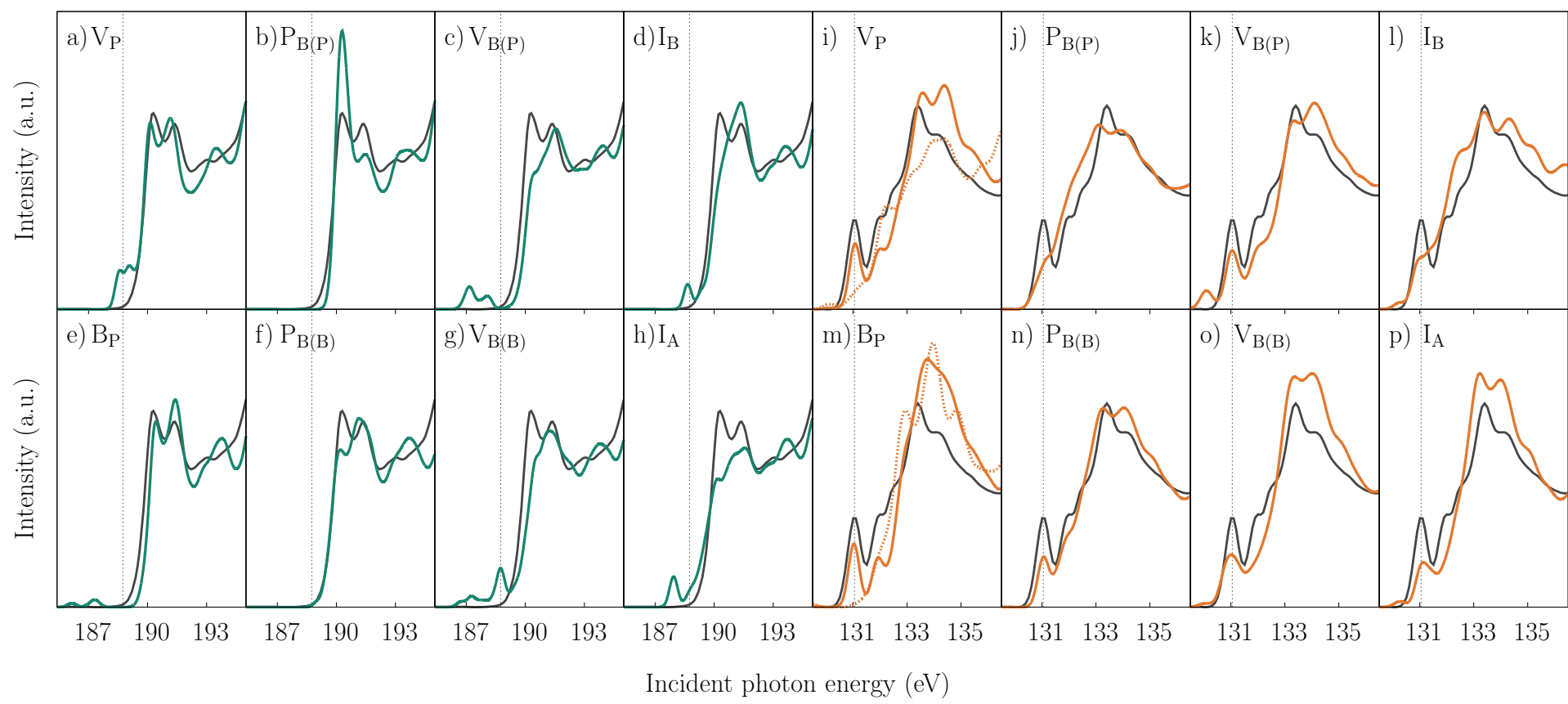

Figure 5.16: Computed x-ray absorption spectra for vacancy, interstitial and antisite defects for the (a-g) B $K$-edge and (h-p) P $L_{2,3}$ edge. The solid black lines represents the experimentally collected spectrum for samples L1 and the colored solid lines are the computed spectra. The computed spectra are the average of the boron and phosphorus atoms within a radius of 3.4 and $4.0 \AA$, respectively, of the point defect. The labels in the top left corner of the images indicate the type of the defect and correspond to the structures and formation energies in Table 5.2. Colored dashed lines, when present, indicate the spectrum of an atom directly neighboring or at the position of the defect. The vertical dashed lines indicate the position of features $A$ in both the $\mathrm{B} K$ and $\mathrm{P} L_{2,3}$ spectra, in correspondence with the labels used in Figs. 6.1 and 5.4 
most intense feature in the B $K$-edge for an unperturbed boron atom in a perfect crystal environment. With an experimental intensity resolution on the approximate order of $1 \times 10^{-2}$ and the fact that for every boron vacancy, five adjacent boron atoms will contribute to these defects states, we expect to be able to resolve a defect concentration of the order of $1 \times 10^{-3}$ to $1 \times 10^{-2}$. Following the same argumentation as for boron vacancies, the presence of $\mathrm{B}_{\mathrm{P}}$ and $\mathrm{I}_{\mathrm{B}}^{\mathrm{A}}$ defects in the samples is also found to be unlikely, since these defects also introduce defect transitions in the x-ray absorption spectra at energies where the experimental absorption is negligible.

The $\mathrm{V}_{\mathrm{P}}, \mathrm{P}_{\mathrm{B}(\mathrm{B})}$ and $\mathrm{I}_{\mathrm{B}}^{\mathrm{B}}$ defects also introduce defect states when neighboring boron atoms are core excited, however, in contrast with the previously discussed defects, these defect states appear at higher energies in the spectrum and overlap exactly with feature $A$ in the B $K$-edge, marked by the dotted line in Fig. 5.16(a)-(g). Feature $A$ can therefore be associated with any one of these defects and the fact that feature $A$ becomes relatively more intense for samples that were deposited at lower temperatures, for which one expects more structural defects, is in good agreement with this prediction. Additionally, the low formation energies for $\mathrm{V}_{\mathrm{P}}$ and $\mathrm{P}_{\mathrm{B}(\mathrm{B})}$ defects (see Table 5.2), show these are the most probable defects under boron and phosphorus-rich conditions, respectively.

Up till now, we determined the presence of point defects by verifying the presence of their characteristic defect transitions that would be absent in the spectrum of the perfect crystal. Analogously, it is also possible to observe the presence of certain defects by studying the suppression of spectral features observed in the spectrum of the perfect crystal, prime examples of which, are the boron antisite and phosphorus vacancy. Both the $\mathrm{B}_{\mathrm{P}}$ and the $\mathrm{V}_{\mathrm{P}}$ defect have the characteristic that the absorption spectrum of the excited phosphorus atom directly next to the antisite or vacancy position, respectively, no longer has the characteristic feature $A$ in the phosphorus $L_{2,3}$-edge. Note that this effect is not directly apparent in the average spectrum, represented by the solid line in Figs. 5.16 (i) and $5.16(\mathrm{~m})$, however the spectrum of the atom directly neighboring the point defect, indicated by the dashed line in the same figures, clearly shows the almost full suppression of feature $A$. Upon closer inspection, this is not suprising at all, since that absorption feature is due to a highly localized $\sigma^{*}$ state on the $\mathrm{P}_{2}$ dimer, as shown in Fig. 5.5, and both the $\mathrm{B}_{\mathrm{P}}$ and $\mathrm{V}_{\mathrm{P}}$ defect effectively destroy the phosphorus dimer and thereby the bond. The relatively decreasing intensity of feature $A$ as observed in experiment for the samples deposited at lower temperatures can therefore be directly linked to presence of boron antisite or phosphorus vacancy defects.

Based on observations made from the $\mathrm{B} K$-edge point defect spectroscopy, it can be concluded that the defects $\mathrm{V}_{\mathrm{P}}, \mathrm{I}_{\mathrm{B}}^{\mathrm{B}}$ and $\mathrm{P}_{\mathrm{B}(\mathrm{B})}$, are potentially present in the samples studied in this work. Analysis of the $\mathrm{P} L_{2,3}$ edge spectroscopy shows that both $\mathrm{V}_{\mathrm{P}}$ and $\mathrm{B}_{\mathrm{P}}$ defects can explain the gradual decrease of feature $A$ observed in the experimental samples deposited at lower temperatures. The fact that the presence of phosphorus vacancies is supported independently by the spectroscopy of both absorption edges 
and that the formation energy of the defect is computed to be the lowest, after the $\mathrm{B}_{\mathrm{P}}$ formation energy, for all considered defects in boron-rich conditions, is evidence that the analyzed samples contain a significant amount of phosphorus vacancies. Determining exact defect quantities is difficult, but the relative difference in the intensity of the $\sigma^{*}$ feature in the $\mathrm{P} L_{2,3}$-edge of the studied samples and the increasing intensity of feature $A$ in the B $K$-edge, would place a careful estimation of phosphorus vacancy abundances on the order of $1 \%$ for the samples deposited at the lower temperatures.

The ability to quantify defect levels with XANES is limited by both the relative intensity of defect states with respect to the spectrum of the bulk, as well as the intensity sensitivity of the experimental setup. The defects discussed in this work typically have associated defect transitions with intensities that are of the same order as bulk transitions and the relative intensity resolution of the experiment is on the approximate order of $1 \times 10^{-3}$ to $1 \times 10^{-2}$. This means that XANES is not a suitable technique to estimate, for example, doping concentrations, which typically have concentrations that are far more dilute. However, our results have clearly shown that x-ray absorption spectroscopy is sensitive to different types of defect populations with a relative concentration of the order of $1 \times 10^{-3}$ or higher, and that relative changes in their abundances can be qualitatively assessed. This technique can therefore prove to be valuable in monitoring defect populations in applications where the relevant material properties are affected negatively by the presence of a significant amount of structural defects.

\subsection{CONCLUSIONS}

We have presented the experimentally collected $\mathrm{x}$-ray absorption spectroscopy of the B $K$ and $\mathrm{P} L_{2,3}$ edge for several $\mathrm{B}_{12} \mathrm{P}_{2}$ samples, grown in a chemical vapor deposition reactor under varying experimental conditions. Simulated x-ray absorption spectra from first-principles, for the perfect crystal at zero and finite temperature, showed great agreement with experiment and facilitated the analysis of spectral features and tracing their origin in the electronic structure of the material. We have discussed point defects as the potential cause for the remaining discrepancies between simulated x-ray absorption spectra for the perfect crystal and the experimental data, as well as the source of the relative difference observed in the spectra for the different samples that were analyzed. To this end, we have simulated the spectroscopic signature of antisite, interstitial and vacancy point defects in both the $\mathrm{B} K$ and $\mathrm{P} L_{2,3}$ edge. The results showed that the relatively increasing intensity of the pre-edge feature in the $\mathrm{B} K$ edge and the relatively decreasing intensity of the first feature in the $\mathrm{P} L_{2,3}$ edge, for samples deposited at decreasing temperatures, both can be explained by the presence of phosphorus vacancies in the samples. Combined with the fact that the computed formation energy of phosphorus vacancies is among the lowest of all the considered 
defects under boron-rich conditions, it is likely that this is the type of defect that is most abundant in the analyzed samples.

Contrastingly, simulations of the x-ray absorption spectroscopy of boron vacancies predict strong absorption features below the main onset of absorption in the $\mathrm{B} K$ edge spectrum, due to the introduction of defect states in the band gap by the defect, which leads to the conclusion that the experimental samples contain little to no boron vacancies. The observed lack or absence of boron vacancies, is reminiscent of the "self-healing" property of icosahedral borides like $\mathrm{B}_{12} \mathrm{P}_{2}$, that has been reported in literature $[15,16]$. Transmission electron microscopy measurements of $\mathrm{B}_{12} \mathrm{P}_{2}$ showed virtually no evidence of amorphization or crystal structure damage, despite having been subjected to intense and highly energetic particle beams. The suggested cause for the observed lack of damage, was the spontaneous recombination of defects, created by the displacement of boron atoms. Recent theoretical work has shown that the activation energies for the recombination of boron vacancies with interstitial boron atoms can be so low that recombination will be spontaneous[84]. This is in strong agreement with the spectroscopic analysis discussed in this work which also finds strong evidence for the lack of boron vacancies. The simulated spectroscopic signatures of point defects, presented in this work, can be used in future studies of the dynamics of defect creation and recombination in $\mathrm{B}_{12} \mathrm{P}_{2}$.

\section{ACKNOWLEDGMENTS}

This work is supported by NanoNextNL, a micro and nanotechnology programme of the Dutch Government and 130 partners. We acknowledge the support of the Center for X-ray Optics of Lawrence Berkeley Laboratory and the Industrial Focus Group XUV Optics at the MESA+ Institute for Nanotechnology at the University of Twente, notably the partners ASML, Carl Zeiss SMT GmbH, and the Foundation FOM. All the computational work was performed at the Molecular Foundry which is supported by the Office of Science, Office of Basic Energy Sciences, of the United States Department of Energy under Contract No. DE-AC02-05CH11231. 


\section{REFERENCES}

[1] S. P. Huber, E. Gullikson, R. W. E. van de Kruijs, F. Bijkerk, and

D. Prendergast, Phys. Rev. B 92 (2015) 10.1103/physrevb.92.245310.

[15] M. Carrard, D. Emin, and L. Zuppiroli, Phys. Rev. B 51, 11270 (1995).

[16] D. Emin, Journal of Solid State Chemistry 179, 2791 (2006).

[17] J. C. Lund, F. Olschner, F. Ahmed, and K. S. Shah, MRS Proc. 162 (1989) 10.1557/proc-162-601.

[18] T. P. Viles, B. A. Brunett, H. Yoon, J. C. Lund, H. Hermon, D. Buchenauer, K. McCarty, M. Clifft, D. Dibble, and R. B. James, MRS Proc. 487 (1997) 10.1557/proc-487-585.

[36] D. Prendergast, and G. Galli, Phys. Rev. Lett. 96, 215502 (2006).

[42] J. Underwood, and E. Gullikson, Journal of Electron Spectroscopy and Related Phenomena 92, 265 (1998).

[43] E. M. Gullikson, S. Mrowka, and B. B. Kaufmann, Recent developments in euv reflectometry at the advanced light source, 2001.

[47] G. Kresse, and J. Furthmüller, Physical Review B 54, 11169 (1996).

[49] E. L. Shirley, Phys. Rev. B 54, 16464 (1996).

[57] I. Jiménez, A. F. Jankowski, L. J. Terminello, D. G. J. Sutherland, J. A. Carlisle, G. L. Doll, W. M. Tong, D. K. Shuh, and F. J. Himpsel, Phys. Rev. B 55, 12025 (1997).

[58] M. Niibe, K. Miyamoto, T. Mitamura, and K. Mochiji, Journal of Vacuum Science \& Technology A 28, 1157 (2010).

[59] I. Caretti, and I. Jiménez, Journal of Applied Physics 110, 023511, (2011).

[60] K. Simonov, N. Vinogradov, M. Ng, A. Vinogradov, N. Mårtensson, and A. Preobrajenski, Surface Science 606, 564 (2012). 
[61] P. E. Blöchl, Physical Review B 50, 17953 (1994).

[62] G. Kresse, and D. Joubert, Physical Review B 59, 1758 (1999).

[63] J. P. Perdew, K. Burke, and M. Ernzerhof, Phys. Rev. Lett. 77, 3865 (1996).

[65] H.-P. Komsa, T. T. Rantala, and A. Pasquarello, Physical Review B 86, 045112 (2012).

[66] C. G. V. de Walle, J. Appl. Phys. 95, 3851 (2004).

[67] A. H. England, A. M. Duffin, C. P. Schwartz, J. S. Uejio, D. Prendergast, and R. J. Saykally, Chemical Physics Letters 514, 187 (2011).

[73] H. J. Monkhorst, and J. D. Pack, Phys. Rev. B 13, 5188 (1976).

[83] Q. An, and W. A. Goddard, Chemistry of Materials 27, 2855 (2015).

[84] S. P. Huber, E. Gullikson, C. D. Frye, J. H. Edgar, R. W. E. van de Kruijs, F. Bijkerk, and D. Prendergast, Unpublished (2016).

[85] P. E. Blöchl, O. Jepsen, and O. K. Andersen, Phys. Rev. B 49, 16223 (1994).

[86] A. V. Krukau, O. A. Vydrov, A. F. Izmaylov, and G. E. Scuseria, The Journal of Chemical Physics 125, 224106 (2006).

[87] Y. Zhang, "Epitaxial growth of icosahedral boron arsenide on silicon carbide substrates: improved process conditions and electrical properties", PhD thesis (Kansas State University, 2011).

[88] D. R. Armstrong, J. Bolland, and P. G. Perkins, Theoretica Chimica Acta 64, 501 (1984).

[89] D. Li, and W. Y. Ching, Phys. Rev. B 52, 17073 (1995).

[90] W. Hayami, and S. Otani, J. Phys. Chem. C 112, 2711 (2008).

[91] G. A. Slack, T. McNelly, and E. Taft, Journal of Physics and Chemistry of Solids 44, 1009 (1983). 

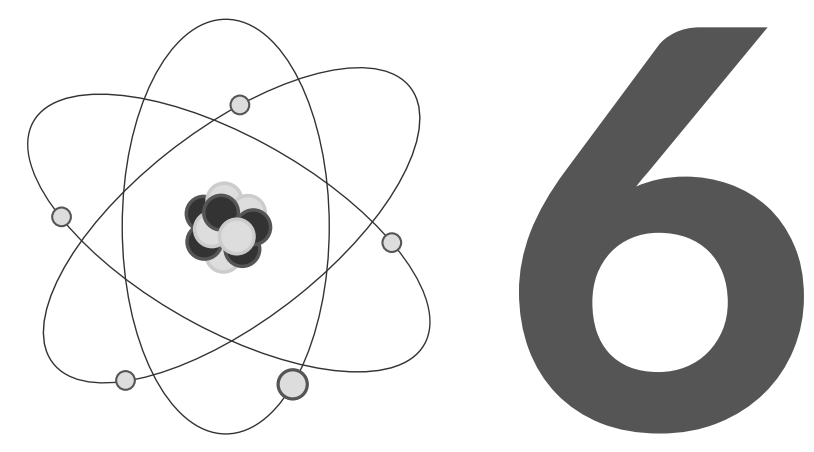

\section{DETERMINING CRYSTAL PHASE PURITY IN CUBIC BORON PHOSPHIDE THROUGH X-RAY ABSORPTION SPECTROSCOPY}




\section{ABSTRACT}

We employ x-ray absorption near-edge spectroscopy at the boron $K$-edge and phosphorus $L_{2,3}$-edge to study the structural properties of cubic boron phosphide $(c$-BP) samples. The x-ray absorption spectra are modeled from first-principles within the density functional theory framework using the excited electron core-hole (XCH) approach. A simple structural model of a perfect $c$-BP crystal accurately reproduces the $\mathrm{P} L_{2,3}$-edge, however it fails to describe the broad and gradual onset of the $\mathrm{B} K$-edge. Simulations of the spectroscopic signatures in boron $1 s$ excitations of intrinsic point defects and the hexagonal BP crystal phase, show that these additions to the structural model can not reproduce the broad pre-edge of the experimental spectrum. Calculated formation enthalpies show that, during the growth of $c$-BP, it is possible that amorphous boron phases can be grown in conjunction with the desired boron phosphide crystalline phase. In combination with experimental and theoretically obtained x-ray absorption spectra for an amorphous boron structure, which have a similar broad absorption onset in the B $K$-edge spectrum as the cubic boron phosphide samples, we provide evidence for the presence of amorphous boron clusters near the surface of the synthesized $c$-BP samples. 


\subsection{INTRODUCTION}

The group III-V compound boron phosphide has been most commonly studied in its cubic zinc blende crystal phase $(c-\mathrm{BP})$, in which it is characterized as a refractory indirect gap semiconductor. Its high thermal conductivity[92], hardness, chemical stability, high neutron capture cross section[23, 92, 93] and electronic structure, make it a promising candidate for application in high-temperature electronic devices, neutron detectors and radiation protective coatings. Boron phosphide has also been proposed for use in the optical elements of next-generation extreme ultraviolet photolithography tools, operating close to the $L_{2,3}$ absorption edge of the phosphorus[22]. Many studies have successfully grown this crystalline phase of boron phosphide; studies to further improve its structural quality are ongoing[23, 24, 92-99].

For the majority of applications of cubic boron phosphide, the structural crystal quality is of great importance, as the useful properties of the materials are often negatively affected by the presence of structural imperfections. These imperfections can range from simple intrinsic crystal point defects, to agglomerations of structural defects and grain boundaries. In principle, it is also possible that other crystal phases, or even amorphous phases, grow alongside the intended phase. To improve the capabilities of current growth methods to grow high quality crystals, it is crucial to be able to analyze and characterize the structural properties of samples synthesized with these methods. In parallel with typical experimental techniques such as x-ray diffraction, to characterize the long range order found in the sample crystal structure, one could use methods that study the structural and chemical properties on a local atomic scale. This is possible with X-ray absorption near-edge spectroscopy (XANES), as it is sensitive to structural point defects in the boron $K$-edge of various boron compounds $[1,3,57]$ and is typically highly sensitive to the different crystal phases of a particular compound. With the recent prediction[100] of the hexagonal phase of boron phosphide $(h$-BP), the subject of several recent studies[101-106], XANES is a promising candidate as a method that will be able to determine the presence of this unintended crystal phase.

In this work, we present experimental x-ray absorption spectroscopy data, collected in the total electron yield (TEY) mode for both the B $K$-edge as well as the $\mathrm{P} L_{2,3^{-}}$ edge, of $c$-BP samples grown through chemical vapor deposition. To the best of the authors' knowledge, to this date neither the boron $K$-edge nor the $\mathrm{P} L_{2,3}$ absorption edges have been reported in literature, either experimentally or theoretically. The experimentally collected spectra are simulated from first-principles within the density functional theory framework in order to model the structure of the grown $c$-BP samples. Our results show that a structural model defined by the perfect cubic crystal structure is able to accurately reproduce the $\mathrm{P} L_{2,3}$-edge but simultaneously fails to describe the B $K$-edge to the same degree of accuracy. We will discuss multiple refinements to the structural model that could hypothetically explain this juxtaposition, such as crystalline point defects and different crystal or amorphous phases. The results provide 


\begin{tabular}{ccccccc}
\hline $\mathrm{ID}$ & $\mathrm{B}_{2} \mathrm{H}_{6}\left(1 \%\right.$ in $\left.\mathrm{H}_{2}\right)$ & $\mathrm{PH}_{3}$ & $\mathrm{H}_{2}$ & $p$ (torr $)$ & $T\left({ }^{\circ} \mathrm{C}\right)$ & $t(\min )$ \\
\hline $\mathrm{S} 1$ & 30 & 80 & 4000 & 700 & 1200 & 30 \\
$\mathrm{~S} 2$ & 30 & 80 & 4000 & 700 & 800 & 5 \\
\hline
\end{tabular}

TABLE 6.1: Experimental conditions during deposition of the two reference samples studied in this work, with partial pressures of precursor gases in sccm, total pressure $p$ in torr, temperature $T$ in ${ }^{\circ} \mathrm{C}$ and deposition time $t$ in min.

strong evidence for the presence of amorphous boron phases incorporated in the cubic boron phosphide crystal structure.

\subsection{EXPERIMENTAL DETAILS}

In this section, we will report on the experimental details of sample deposition and the experimental x-ray absorption spectroscopy measurements of the boron $K$-edge and phosphorus $L_{2,3}$-edge, performed at the Advanced Light Source (ALS).

\subsubsection{CUBIC BORON PHOSPHIDE SAMPLE DEPOSITION}

The two cubic boron phosphide samples analyzed in this work were deposited onto $4 H$-SiC(0001) substrates by chemical vapor deposition (CVD). The CVD setup and experimental methods used in this work have been described in greater detail elsewhere[23, 24, 92]. The precursor gases were ultra-high purity phosphine $(99.999 \%)$ and diborane $\left(1 \%\right.$ in $\left.\mathrm{H}_{2}\right)$ in an ultra-high purity hydrogen carrier gas. Detailed deposition conditions employed are listed in Table 6.1. The deposition temperature and precursor gas flow rates were adjusted accordingly to achieve a highly crystalline structure (S1) and an amorphous structure S2, which was verified by scanning electron microscopy and x-ray diffraction analysis.

\subsubsection{BORON AND BORON NITRIDE SAMPLE DEPOSITION}

To compare structural and x-ray absorption spectroscopy properties of the cubic boron phosphide samples, we also deposited boron and boron nitride samples as a reference. These reference samples were deposited in a ultra-high vacuum (UHV) chamber with base pressure of $1 \times 10^{-8}$ mbar, using electron-beam evaporation of a boron target onto the native oxide of single crystalline silicon substrates. The target thickness was approximately $40 \mathrm{~nm}$ for both samples, monitored by quartz mass balances, which typically yields an uncertainty of $10 \%$ in the final film thickness. For the growth of the boron nitride sample, a repetitive process was employed where first a thin layer of $0.7 \mathrm{~nm}$ boron was deposited, which was subsequently treated with nitrogen ion implantation 
for $240 \mathrm{~s}$ to create boron nitride. Nitrogen ions were produced with a Kaufman type hot cathode ion source operated at $60 \mathrm{~mA}$ and $150 \mathrm{~V}$. Post-deposition x-ray photoelectron spectroscopy (XPS) analysis of the boron nitride sample revealed that all the boron had reacted with the nitrogen and no elementary boron remained. The sample substrate holder remained near room temperature during the deposition process and the structure of the grown samples were determined to be amorphous by post deposition $\mathrm{x}$-ray diffraction analysis.

\subsubsection{TOTAL ELECTRON YIELD X-RAY ABSORPTION SPECTROSCOPY}

All x-ray absorption spectroscopy measurements were carried out at beamline 6.3.2 of the Advanced Light Source (ALS) synchrotron at Lawrence Berkeley National Laboratory (LBNL). A detailed description and characterization of the beamline and measurement chamber can be found elsewhere[42, 43]. X-ray absorption measurements of the boron $K$-edge and phosphorus $L_{2,3}$ edge, were collected in total electron yield (TEY) mode. The linearly polarized incident soft x-ray beam was oriented parallel to the sample surface normal. Photon energies were calibrated by comparing to absolute absorption edges of $\mathrm{Si}$ and $\mathrm{B}$ filters installed at the beamline. The collected spectra have the dark current signal subtracted to account for the systematic error and noise in the collector electronics. Subsequently, the spectra are normalized by a spectrum collected by a photodiode to account for the intensity fluctuations in the x-ray beam as a function of photon energy.

\subsection{COMPUTATIONAL METHOD}

\subsubsection{X-RAY ABSORPTION SPECTROSCOPY}

X-ray absorption spectroscopy (XAS) was simulated within the density functional theory excited electron and core-hole (DFT-XCH) approach[36] where the photo excited atom is modeled by removing a core electron during the generation of the corresponding excited state pseudopotential and placing it in the first available empty state in subsequent valence electron calculations. The electronic structure problem, with this core-hole occupation constraint, is solved self-consistently. Non-self-consistent band structures and associated eigenstates are generated efficiently for numerical convergence in Brillouin zone integrations using the Shirley interpolation scheme[49]. Plane waves were expanded up to a maximum of $30 \mathrm{Ry}$ and ultrasoft pseuodpotentials $[107$, 108 ] were employed. The absorption spectrum is computed by evaluating the transition probability between the initial and final state as given by Fermi's golden rule within the dipole approximation. The resulting spectrum is broadened uniformly with a Gaussian of $0.2 \mathrm{eV}$ FWHM for all structural models. To correct for the well-known underestimation of the band gap and band width by local or semilocal functionals, such 
as the PBE functional, the energy scale is stretched uniformly by a factor of 1.08 for the phosphorus $L_{2,3}$-edge of all boron phosphide structures, both crystalline and amorphous. For the boron $K$-edge, in all boron and boron nitride structures, a stretching factor of 1.08 and 1.04 is used, respectively.

Due to the lack of an absolute energy reference inherent in the pseudopotential method, an energy alignment scheme was employed to yield comparably meaningful relative energies for structurally and chemically different systems[67]. This approach does not necessarily yield a perfect relative alignment, as is the case for the systems studied here, which will be addressed in greater detail later in this work. Finally, the spectra were shifted by a single value, which was kept identical for all computed spectra of one particular compound, regardless of its crystal phase or structure. The compound specific value was obtained by comparing the computed x-ray absorption spectrum for the perfect crystal structure with the corresponding experimental spectrum. In the case of an $L_{2,3}$-edge transition, electrons from either the $2 p_{3 / 2}$ or $2 p_{1 / 2}$ core level can be excited, which, due to spin-orbit coupling, are energetically split. Under the assumption that excitations from $2 p_{3 / 2}$ or $2 p_{1 / 2}$ core levels are equally probable, in this work, this effect is accounted for by adding the spectrum to itself, after shifting it by an energy $\Delta E$ equal to the spin-orbit splitting of the relevant element, and properly weighting the two spectra by a ratio of $2: 1$ that reflects the population ratio of the $2 p_{3 / 2}$ and $2 p_{1 / 2}$ core levels.

\subsubsection{STRUCTURAL OPTIMIZATIONS}

All structural optimizations were performed within the density functional theory (DFT) framework as implemented in the Vienna $a b$ initio simulation package vasP[47]. The DFT computations were performed using a generalized gradient approximation (GGA) functional parametrized by Perdew, Burke, and Ernzerhof (PBE)[63] and ultrasoft pseudopotentials within the projector augmented wave (PAW) method[61, 62], where the $2 s$ and $2 p$ electrons for boron and the $3 s$ and $3 p$ electrons for phosphorus are treated as valence electrons. Plane waves, to represent the wave functions, were expanded up to an energy of $400 \mathrm{eV}$ and the Brillouin zone was sampled at the $\Gamma$ point. The condition for structural relaxation was defined as a maximum Hellmann-Feynman force of $0.02 \mathrm{eV}^{-1}$ acting on any individual nucleus.

\subsubsection{CRYSTAL CELL DEFINITIONS AND MOLECULAR DYNAMICS}

The crystalline $\alpha$-rhombohedral boron $(\alpha$-B), hexagonal boron nitride $(h$-BN), hexagonal boron phosphide $(h$-BP) and cubic boron phosphide $(c$-BP) structures, were constructed as $2 \times 2 \times 2,4 \times 4 \times 2,4 \times 4 \times 2$ and $3 \times 3 \times 3$ supercells from the corresponding crystalline unit cell, respectively. The resulting $\alpha$-B, $h$-BN, $h$-BP and $c$-BP supercells had a total of 288, 128, 128 and 216 atoms, respectively. Finite temperature effects on the 
crystal structures were simulated by sampling the canonical ensemble $(N V T)$ at a temperature of $300 \mathrm{~K}$ regulated by a Nosé-Hoover thermostat. The time step of integration was set to $0.2 \mathrm{fs}$ for all crystal structures. The systems were thermally equilibrated for at least $5 \mathrm{ps}$, after which the micro canonical ensemble $(N V E)$ was sampled, taking at least 2 snapshots, separated by at least 1 ps. The calculated x-ray absorption spectrum for each crystal structure is defined as the average of the spectra computed for each of the snapshots taken from the corresponding equilibrated molecular dynamics trajectory. For the planar hexagonal structures, van der Waals interactions were accounted for by applying the corrective scheme of Tkatchenko and Scheffler[64] as implemented in VASP.

\subsubsection{SIMULATION OF AMORPHOUS STRUCTURES}

All amorphous structures discussed in this work were simulated using first-principles molecular dynamics methods implemented in the aforementioned software package VASP. The overall approach was identical for all generated amorphous networks and consisted largely of three consecutive steps. Firstly, the initial crystal structure was heated instantaneously to temperatures well above the experimental melting temperature of the respective crystal phase, by coupling the system to a Nosé-Hoover thermostat. The NVT ensemble was sampled at the elevated temperature until a structural defect arose that was strong enough to break the crystal symmetry and initiate the melting process of the crystal structure, which typically took between 200 fs to $4000 \mathrm{fs}$. The liquid was sampled at the elevated temperature for at least 3 ps to allow for significant atomic diffusion to occur, after which the temperature was gradually reduced to $300 \mathrm{~K}$ at a rate of approximately $1 \times 10^{-15} \mathrm{~K} \mathrm{~s}^{-1}$. After quenching from the liquid, the amorphous structure was equilibrated at $300 \mathrm{~K}$ for at least $3 \mathrm{ps}$ before the trajectory was sampled for snapshots or structural properties. The initial crystal cells that were used for the simulation of the amorphous boron $(a-\mathrm{B})$, boron nitride $(a$-BN) and boron phosphide $(a$-BP) structures, were the crystalline supercells described in the previous section, consisting of 288, 128 and 216 atoms, respectively.

\subsection{EXPERIMENTAL RESULTS}

\subsubsection{X-RAY ABSORPTION SPECTROSCOPY}

For each of the samples listed in Table 6.1, the x-ray absorption spectrum, at both the boron $K$-edge and the phosphorus $L_{2,3}$-edge, has been collected in the total electron yield (TEY) mode, as shown in Fig. 6.1. The area under the curves has been normalized to unity to enable meaningful comparison of relative changes in intensity of certain features. 

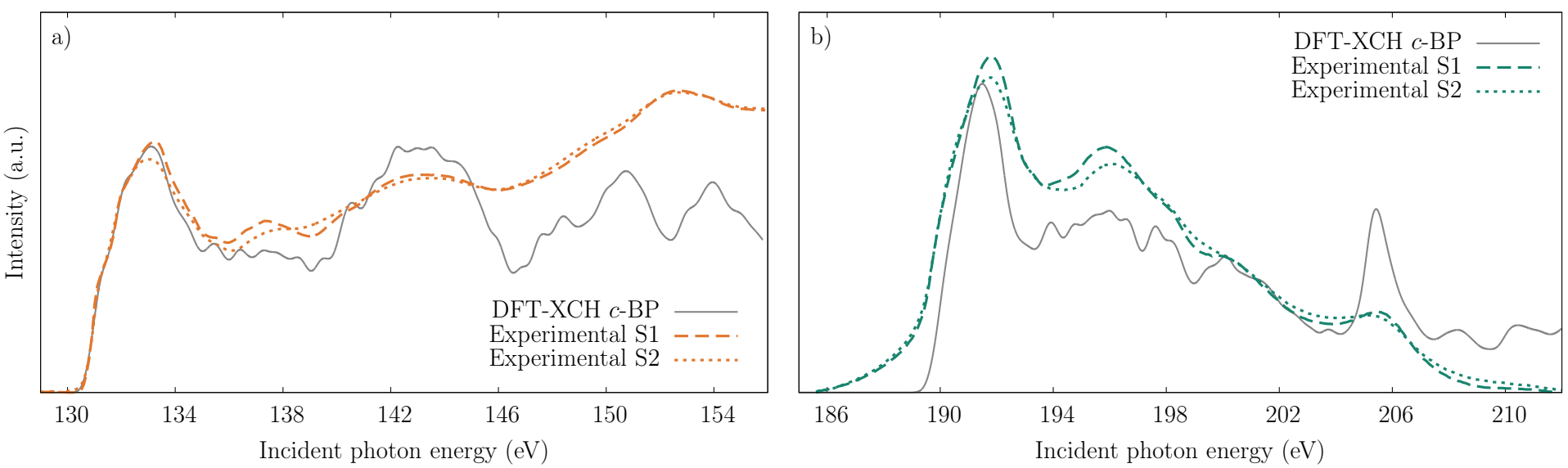

Figure 6.1: The dashed colored lines represent the experimentally collected x-ray absorption spectra for the (a) phosphorus $L_{2,3}$-edge and the (b) boron $K$-edge for the two reference $c$-BP samples, obtained in the total electron yield mode. The solid black line in both panels corresponds to the simulated spectrum, computed with the DFT-XCH method for the perfect $c$-BP crystal structure, equilibrated at room temperature. 
Despite the significant difference in experimental conditions, under which the two BP reference samples were deposited, specifically the difference in temperature, the collected spectra for both absorption edges are remarkably similar. The main discrepancy between the spectra of the two samples is the slight broadening of the spectral features for sample S2 compared to S1. Since XANES probes the chemical and structural environment in the direct vicinity of the excited atom, it is insensitive to long range amorphous or crystal structures. However, distortions of the perfect crystal lattice, as in for example amorphous phases, will also distort local structural properties, like bond angles and bond lengths. Distortions from the equilibrium structure on the local scale, induced by amorphization, are reflected in the x-ray absorption spectra by the broadening of the characteristic crystalline features. These broadening effects are partly due to new electronic transitions, induced by the structural defects of the crystal lattice, and are therefore not necessarily symmetrical or uniformly distributed, as would be the case in for example diffraction experiments. The broadening of features in the spectra for sample S2 can hence be attributed to the presence of amorphous phases, however it should be emphasized that the results show that the short range structural properties remain predominantly and distinctively cubic, as will be shown in the following section.

\subsection{COMPUTATIONAL RESULTS}

The solid black lines in Fig. 6.1 represent the simulated x-ray absorption spectra for the perfect $c$-BP crystal thermalized at a temperature of $300 \mathrm{~K}$. Overall the simulated spectra agree well with experiment for both the $\mathrm{B} K$-edge as well as the $\mathrm{P} L_{2,3}$ edge. The experimental features are generally smoother compared to the theory, which can be attributed to fact that the experimental samples are not perfect crystals and slight distortions from the perfect lattice lead to a broadening of the typical crystalline spectral features.

The DFT-XCH method used in the simulations, possesses the highest fidelity close to the absorption edge and will become less trustworthy for higher excitation energies. Discrepancies between experiment and theory close to the absorption edge are for that reason of most interest and therefore the most significant discrepancy in the data, is the absorption onset of the B $K$-edge. The theory models a sharp and sudden onset of the absorption edge, whereas the spectra collected for both samples S1 and S2, show a broad and gradual onset. In the following sections we will discuss several hypotheses that could potentially explain the discrepancy in the predicted and measured B $K$-edge, one of which is the presence of crystal point defects in the experimental samples. 


\subsubsection{X-RAY ABSORPTION SPECTROSCOPY OF POINT DEFECTS}

X-ray absorption spectroscopy of the $\mathrm{B} K$-edge is sensitive to point defects of the crystal structure in various boron compounds $[1,3,57-60]$. To determine whether point defects are capable of explaining the broad onset of absorption observed in experiment, we have computed the x-ray absorption spectra for several point defects. We consider boron antisite $B_{P}$, phosphorus antisite $P_{B}$, boron vacancy $V_{B}$ and phosphorus vacancy $V_{P}$ defects. The simulated $\mathrm{P} L_{2,3}$ and $\mathrm{B} K$-edge spectra for the four different point defects are shown in Fig. 6.2.

As a reference, the experimental spectrum of sample S1 is shown as orange or green solid line for the $\mathrm{P} L_{2,3}$ and $\mathrm{B} K$-edge, respectively. The solid black line represents the spectrum obtained by averaging the computed x-ray absorption spectra for every possible system with its excited atom located within a radius of $3.8 \AA$ of the point defect, which, depending on the specific defect and absorption edge, ranges between 12 and 17 atoms. For each defect, an individual atomic spectrum is also shown with a black dashed line, which corresponds to an atom closest to the defect, in the case of a vacancy defect, or of the defect atom itself in the case of an antisite defect. Note that, in the $\mathrm{P} L_{2,3}$ spectra the $\mathrm{V}_{\mathrm{P}}$ and $\mathrm{B}_{\mathrm{P}}$, and likewise in the $\mathrm{B} K$-edge spectra for the $\mathrm{V}_{\mathrm{B}}$ and $\mathrm{P}_{\mathrm{B}}$, these indiviual atomic defect spectra are not visible as they overlap directly with their corresponding average spectrum. In these specific cases, all atoms considered in the average, are symmetrically identical with respect to the defect, due to the radial symmetry of the crystal.

The boron vacancy introduces a defect state and corresponding defect transition in the boron $K$-edge at around $189 \mathrm{eV}$, as evidenced by the small pre-edge feature in Fig. 6.2(e). The intensity of the feature is rather low since the closest boron atoms to the vacancy are approximately at a distance of $3.25 \AA$. The effect on the spectrum of the phosphorus atoms directly adjacent to the vacancy, is much larger due to the proximity to the defect, and a significant contribution to the pre-edge in the $\mathrm{P} L_{2,3}$ spectrum can be seen in Fig.6.2(a). This pre-edge feature is not observed in the experimental $\mathrm{P} L_{2,3}$ spectra, and since the effects of boron vacancies on both edges are intrinsically connected and cannot be regarded independently from one another, the hypothesis of boron vacancies causing the broad absorption onset in the experimental B $K$-edge, has to be rejected.

The same conclusion can be drawn for the phosphorus antisite $\mathrm{P}_{\mathrm{B}}$, but for opposite reasons. As Fig. $6.2(\mathrm{~d})$ shows, the defect does not introduce any defect transitions in the $\mathrm{P} L_{2,3}$ edge, below the absorption edge found in experiment, that could disqualify it. However, it also fails to explain the broad absorption edge of the B $K$-edge, as can be seen in Fig. 6.2 (h). The defects $\mathrm{V}_{\mathrm{P}}$ and $\mathrm{B}_{\mathrm{P}}$ both introduce defect transitions in the $\mathrm{B} K$-edge, which in the case of $\mathrm{V}_{\mathrm{P}}$ leads to a particularly intense feature due to the proximity of four atoms directly adjacent to the defect. The $\mathrm{P} L_{2,3}$ edge is not affected in a way that would discount these defects, except for a slight pre-edge feature 

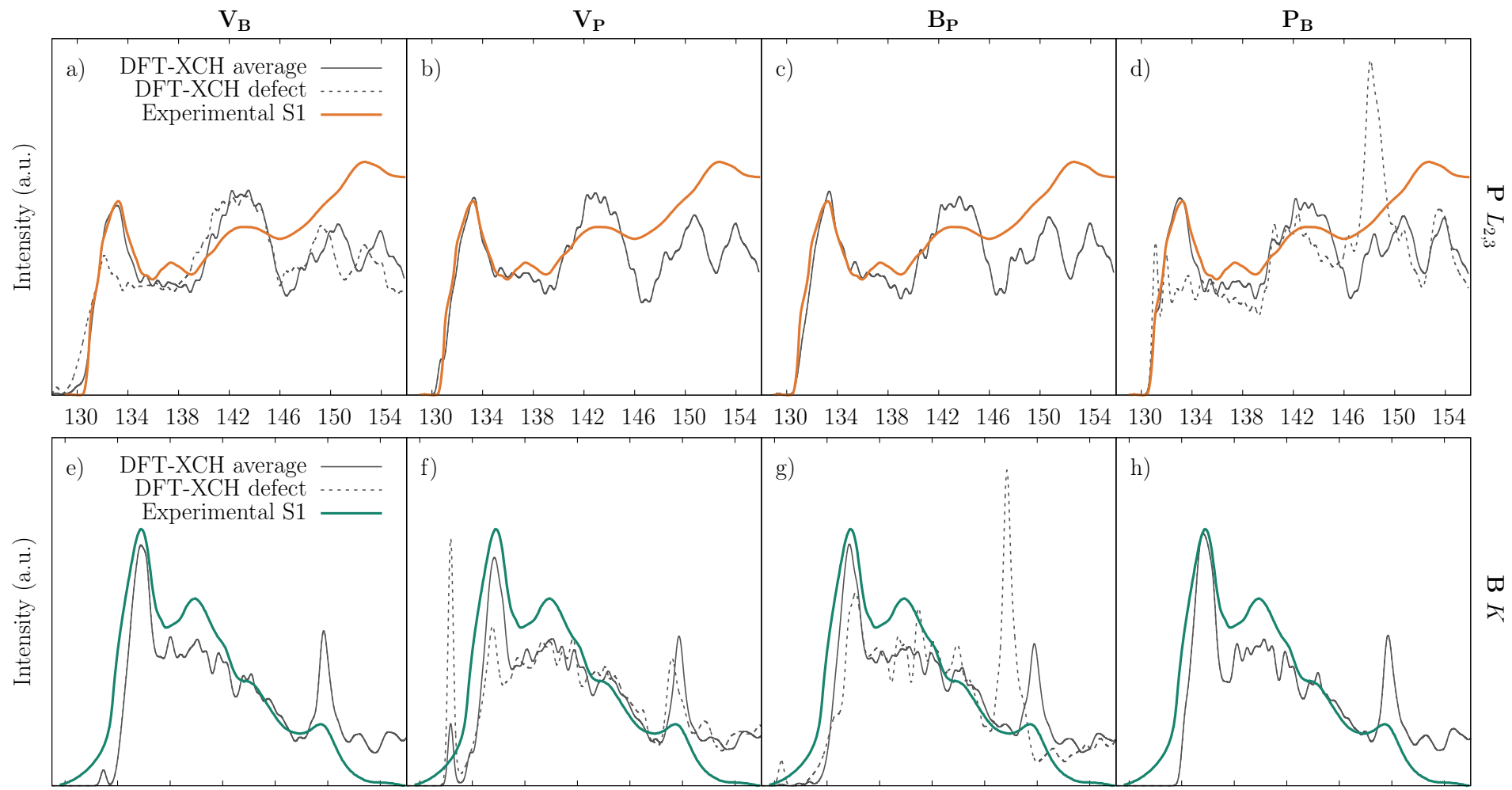

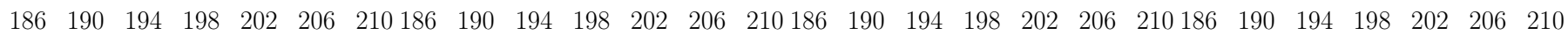
Incident photon energy $(\mathrm{eV})$

Figure 6.2: Computed spectroscopic signatures in the $\mathrm{P} L_{2,3}$-edge [B $K$-edge] of a (a)[e] boron vacancy $\left(\mathrm{V}_{\mathrm{B}}\right)$, (b) [f] phosphorus vacancy $\left(\mathrm{V}_{\mathrm{B}}\right),(\mathrm{c})[\mathrm{g}]$ boron antisite $\left(\mathrm{B}_{\mathrm{P}}\right)$ and $(\mathrm{d})[\mathrm{h}]$ phosphorus antisite $\left(\mathrm{P}_{\mathrm{B}}\right)$ point defect in a $c$-BP crystal. The solid black line represents the spectrum obtained by averaging the computed x-ray absorption spectra for every possible system with its excited atom located within a radius of $3.8 \AA$ of the point defect. The dashed black line represents the computed spectrum for the excited system where the excited atom is the atom that constitutes the point defect or the atom directly adjacent to the defect. 
that occurs in the $\mathrm{P} L_{2,3}$ edge for the $\mathrm{V}_{\mathrm{P}}$ defect, however its distance in energy with respect to the experimental absorption edge is within the experimental uncertainty due to intrinsic and instrumental broadening.

In summary, only $\mathrm{V}_{\mathrm{P}}$ and $\mathrm{B}_{\mathrm{P}}$ defects are predicted to introduce defect transitions in the B $K$-edge, at energies that overlap with the experimentally observed broad absorption pre-edge feature, while not introducing defect transitions in the $\mathrm{P} L_{2,3}$ spectrum that are not observed in experiment. However, the width of the defect transitions in the B $K$-edge are distinctively narrow compared to the broad absorption onset found in the experiment. Even a linear combination of the different defects and their corresponding defect features is unlikely to result in such a smooth broad absorption onset. Spectral broadening in the DFT-XCH method is artificially simulated by the uniform linewidth broadening of individual defect states at a fixed width of $0.2 \mathrm{eV}$, which is an empircal value. This value accurately models the broadening of various defect states in boron compounds, such as boron vacancies and oxygen substitutional defects in $h$-BN[1], as well as phosphorus vacancies in the icosahedral boron phosphide $\mathrm{B}_{12} \mathrm{P}_{2}[3]$. Even though the defect spectroscopy was simulated at a temperature of $0 \mathrm{~K}$ and spectral broadening can in principle be influenced by thermal structural distortions induced by a finite temperature, we have verified that the broadening of these defect transitions reamins unchanged at a finite temperature of $300 \mathrm{~K}$. Since the defect states, associated with the structural defects, are single excited states located in the band gap, which are therefore strongly localized, they are insensitive to perturbations of the local atomic structure. It therefore seems unlikely that the broad absorption onset in the B $K$-edge can be explained by the presence of antisite and or vacancy point defects.

\subsubsection{X-RAY ABSORPTION SPECTROSCOPY OF DIFFERENT CRYSTAL PHASES}

Another potential explanantion for the inability of the perfect cubic crystal lattice to reproduce the experimentally determined absorption onset, is that the experimental sample contained multiple crystal phases. A closely related material to boron phosphide is boron nitride, which, in addition to its cubic phase, has at least one other stable phase; the hexagonal phase. The boron $K$-edge for both the cubic $(c-\mathrm{BN})$ and hexagonal $(h$-BN) phase of boron nitride have been studied extensively in the literature and are well understood. The hexagonal phase has characteristic trigonal bonding, whereas the bonding in the cubic phase is tetragonal and therefore, given the sensitivity of XAS to local structure and chemistry, the x-ray absorption spectra for these two phases are distinct, as shown in Fig. 6.3 (a).

The trigonal bonding in $h$-BN [dashed blue line in Fig. 6.3 (a)] gives rise to a strong isolated pre-edge absorption feature at $192 \mathrm{eV}$, due to a highly localized excitonic state with $\pi^{*}$ character[1], which is located below the absorption edge of the spectrum of $c$-BN, indicated by the solid blue line in the same figure. Due to the many parallels that can be drawn between boron nitride and boron phosphide, it is reasonable to 


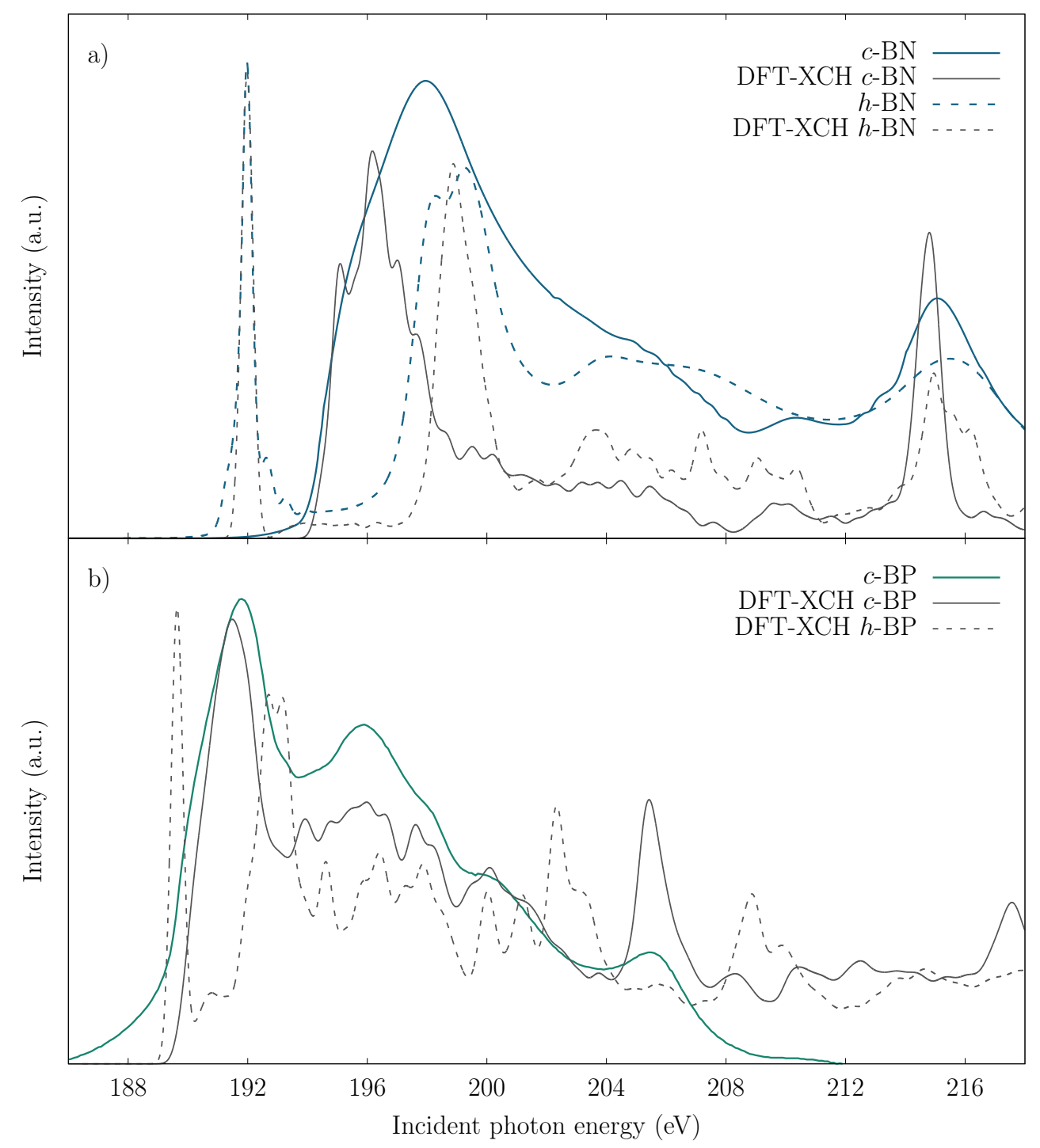

FiguRE 6.3: Experimental and theoretical boron $K$-edge absorption spectroscopy of (a) boron nitride and (b) boron phosphide. The colored lines correspond to experimental data and the black lines are results obtained from calculations following the DFT-XCH method. Solid lines correspond to absorption spectra for cubic phases, whereas the dashed lines represent absorption spectra of hexagonal phases. The experimental data for the $h$-BN and $c$-BN phases were obtained from Huber et al.[1] and McCulloch et al.[109], respectively. 

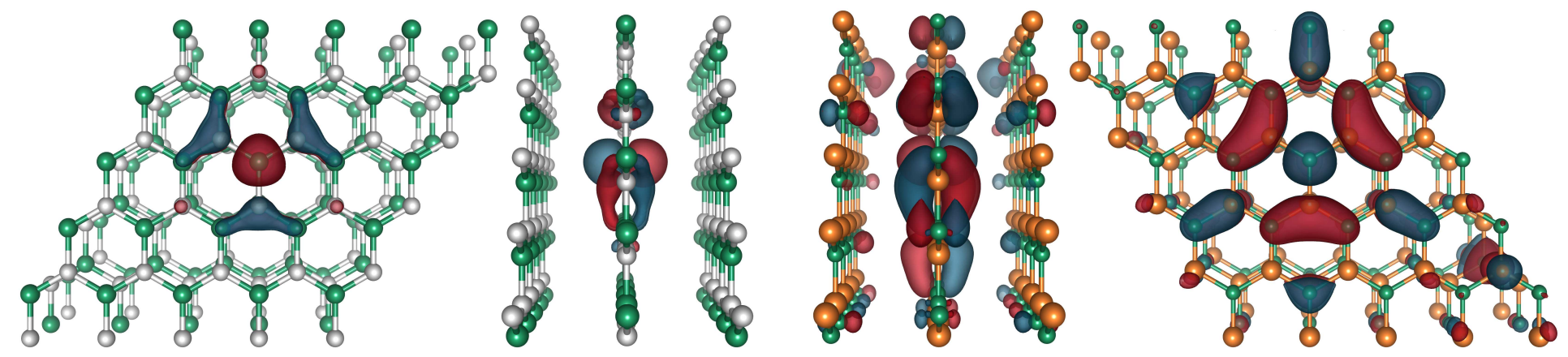

FiguRE 6.4: From left to right, a front and side view of an isosurface of the squared wavefunction $|\psi(\vec{r})|^{2}$ of the highest occupied state for a boron $1 s$ excitation in $h$-BN and, in reverse order, in $h$-BP. The values of the isosurface drawn is chosen such that $50 \%$ of the total charge density norm is contained within the surface. The excited states in $h$-BN and $h$-BP both have a strong $\pi^{*}$ character, however the state in $h$-BN is clearly more localized compared to the same state in $h$-BP. The two different colors of the isosurfaces represent the phase of the wave function in that region. 
hypothesize that the hexagonal boron phosphide phase will have similar absorption spectroscopy characteristics and, like $h$-BN, will have a strong absorption feature below the main absorption edge of its tetragonally bonded cubic counterpart. Since $h$-BP has not yet been successfully synthesized in isolated form, no experimental data exists for its boron $K$-edge absorption spectrum, however, it can be predicted employing the DFT-XCH approach, given the success of said method in simulating and characterizing the B $K$-edge of $h$-BN.

The computed spectrum of the boron $K$-edge for a perfect $h$-BP crystal is shown in Fig. 6.3 (b). It bears a strong resemblence to that of the $h$-BN crystal structure, as it also has a strong isolated $\pi^{*}$ feature, followed by a broader feature resulting from excitations into electronic states with $\sigma^{*}$ character, albeit it red-shifted by at least $2 \mathrm{eV}$ and contracted significantly, as evident in the reduced separation between the $\pi^{*}$ and $\sigma^{*}$ peaks. There is no experimental reference for $h$-BP to permit energy alignment of the computed spectrum and employing the same energy shift used in the alignment of $c$-BP may not necessarily be correct. The energy aligment scheme employed in this work[67], is designed to reference spectra of different structures but of similar chemical nature, to a single relative energy scale. In theory, a single constant value can then be used to align the computed x-ray absorption spectra for all structural configurations with respect to experiment. However, this method does not account for the presence of differing self-interaction errors in the computed energies of the highest occupied electronic orbital in the total energy of the core-hole excited state of the system, which are used directly in the alignment scheme.

As an example, due to the highly localized character of the first excited state in $h$-BN compared to the first excited state in $c$-BN, the self-interaction error is much larger in the former and therefore, accurate relative alignment consistent with experiment requires two different absolute alignment energy shifts of $191.5 \mathrm{eV}$ and $190.7 \mathrm{eV}$, respectively. Even though it is currently not possible to determine the correct absolute energy shift for $h$-BP, it should be smaller than the shift required for $h$-BN, since the first core-excited state used in the relative alignment is much more localized for $h$-BN as compared to $h$-BP. The difference in localization of the highest occupied electronic orbital for a boron $1 s$ excited state of $h$-BN and $h$-BP, is visualized in Fig. 6.4. Nevertheless, we use the same value of $\Delta E$ for $h$-BP as the one used for the alignment of the $h$-BN spectrum to experiment, which guarantess an upper limit for the required absolute energy shift in the $h$-BP spectrum. In other words, we would expect the true $h$-BP boron $K$-edge to be slightly red-shifted with respect to the computed spectrum in Fig. $6.3(\mathrm{~b})$.

Under the uncertainties in absolute energy alignment in the computed $h$-BP spectrum, it is still possible that the $\pi^{*}$ excitation overlaps with the absorption onset of $c$-BP. However, the width of the $\pi^{*}$ feature is rather narrow and, reminiscent of the spectroscopic signatures of point defects discussed in Section 6.5.1, and such narrow 
spectroscopic features are unlikely to give rise to such a smooth broad absorption edge observed in the exprerimental XAS for $c$-BP.

A structural property that can affect the broadening of absorption spectral features is the degree of crystallinity. Even though x-ray absorption spectroscopy probes the local structure of a specific element and is therefore insensitive to long range order, a loss of crystallinity will also affect characteristic structural properties on a local scale. Amorphous phases may still predominantly possess a local bond order that resembles that of the perfect crystal, however the disruption of the long range order will cause typical values of structural properties, such as bond lengths and bond angles, to obtain wider distributions. Spectral absorption features that directly correlate with these structural quantities will therefore experience a similar broadening.

\subsubsection{X-RAY ABSORPTION SPECTROSCOPY OF AMORPHOUS PHASES}

Once again, a suitable example to demonstrate the effect of amorphization of a material on its x-ray absorption spectroscopy, is hexagonal boron nitride. As a reference, Fig. 6.5 (a) depicts the B $K$-edge spectrum of the poly-crystalline $h$-BN sample, that is also shown in Fig. 6.3 (a) and discussed in detail in the corresponding section. Figure 6.5 (b) depicts the B $K$-edge x-ray absorption spectrum collected in TEY mode for a boron nitride sample deposited with electron-beam evaporative physical vapor deposition (see Section 6.2.2 for deposition details). Due to the low temperature during deposition, the structural character of this stochiometric boron nitride sample, is characterized not by crystalline but amorphous phases.

To model the absorption spectrum of the amorphous boron nitride sample, we have simulated an amorphous BN structure with a method implemented in ab initio molecular dynamics, details of which can be found in Section 6.3.4. Starting from the perfect crystal, a liquid was generated by elevating the temperature above the melting point, allowing the system to diffuse. The melt was subsequently quenched and brought to room temperature, where the resulting amorphous network was equilibrated at a constant temperature of approximately $300 \mathrm{~K}$. From the equilibrated trajectory, multiple snapshots were taken, for which the x-ray absorption spectrum was calculated. An example of such a snapshot, taken from the amorphous boron nitride trajectory is shown in Fig. 6.6 (a). The average of the absorption spectra computed for all the configurational snapshots is plotted in Fig. 6.5 (b) as the solid green line. Comparing the experimental x-ray absorption spectra in Figs. 6.5 (a) and 6.5(b), the changes in the boron $K$-edge, resulting from the amorphization of the hexagonal crystal structure, are captured well by the structural model of the amorphous boron nitride network. Overall, the characteristic spectral shape of the $h$-BN lattice is maintained, but the spectrum is significantly broadened. As mentioned before, this indicates that locally the trigonal bonding characteristic of the hexagonal lattice is maintained, but that 

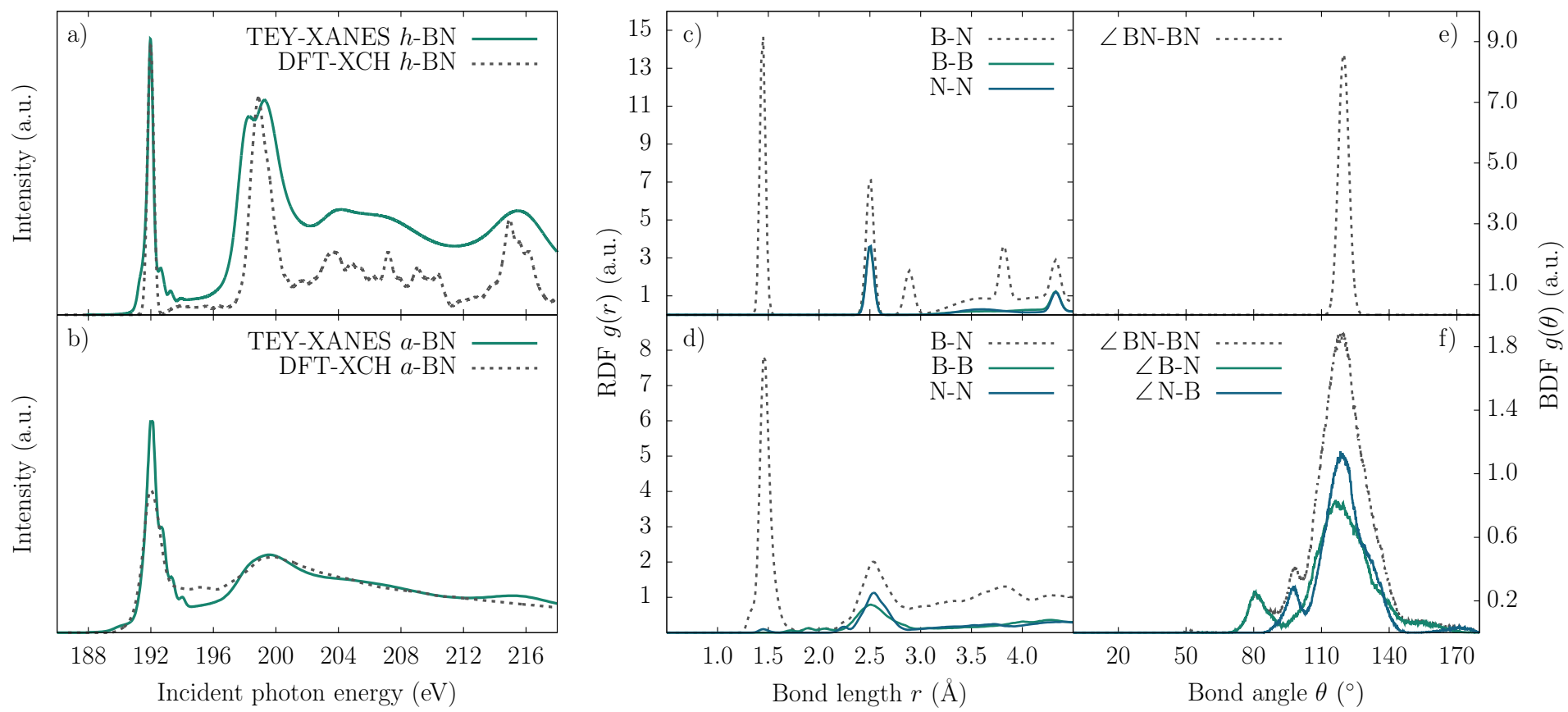

FiguRE 6.5: Experimental and theoretical boron $K$-edge x-ray absorption spectra for (a) crystalline ( $h$-BN) and (b) amorphous boron nitride $(a-\mathrm{BN})$. The solid green lines represent the experimentally collected XAS in TEY mode, whereas the dashed grey lines correspond to the computed spectrum for (a) the perfect crystal and (b) the amorphous network, respectively, both equilibrated around $300 \mathrm{~K}$. Panels (c) and (d) display the average radial distribution function for the equilibration trajectory of the crystal and amorphous structure, respectively. The dashed lines represent the distribution of the distance between both boron and nitrogen atoms, whereas the green and blue solid lines represent the homogeneous atomic distance distribution of boron and nitrogen atoms, respectively. Panels (e) and (f) display the average bond angle distribtution for the same molecular dynamics trajectories as described by the radial distribution function in panels (c) and (d). The dashed gray, solid green and solid blue line are calculated bond angle distributions for bond lengths up to $1.6 \AA$ and $1.8 \AA$ for figure (e) and (f), respectively. 

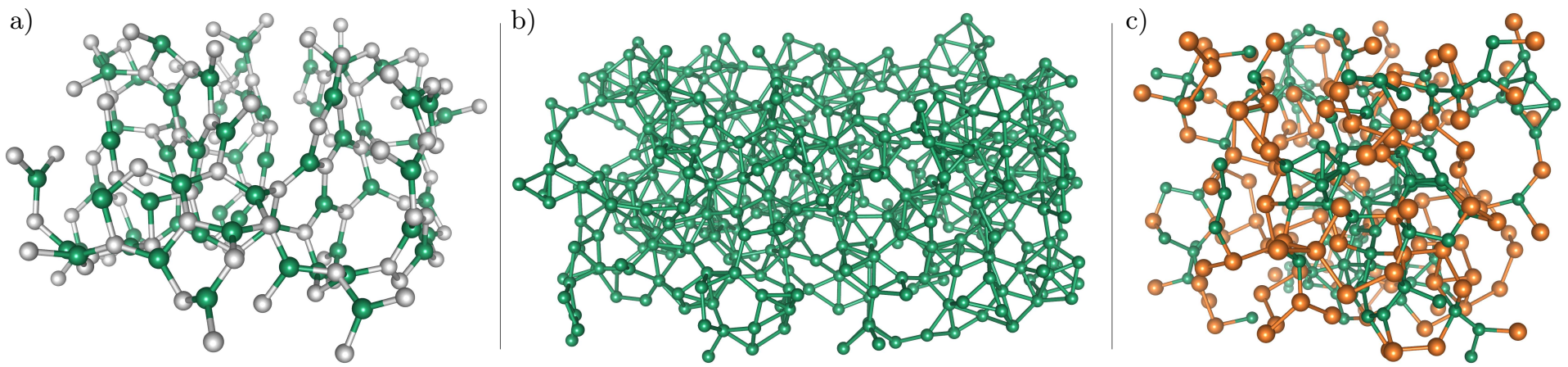

Figure 6.6: Snapshots from a molecular dynamics trajectory of an (a) amorphous boron nitride, (b) amorphous boron and (c) amorphous boron phosphide structure, equilibrated at approximately $300 \mathrm{~K}$. Boron, nitrogen and phosphorus atoms are represented by green, gray and orange spheres, respectively. Boron-boron, boron-nitrogen and boron-phosphorus bonds are drawn up to maximum distance of 1.8 , 2.0 and $2.4 \AA$, respectively. 
typical bond lengths and angles are distorted from the optimal equilibrium values due to the absence of the long range order of a crystal lattice.

Structural differences between the crystal and amorphous configurations can be quantified by the radial distribution and bond angle distribution functions. The radial distribution function (RDF) of the simulated thermalized $h$-BN crystal structure and the amorphous BN network, shown in Figs. 6.5 (c) and 6.5(d), respectively, represents the probability $g(r)$ of finding an atom at a distance $r$ from another atom. The dashed line considers both boron and nitrogen atoms and features a sharp peak around $1.46 \AA$ which is the characteristic bond length found in $h$-BN. The solid green and blue lines are defined similarly but only consider homogeneous boron or nitrogen bonds, respectively, and an absence of their contribution at the first-nearest neighbor peak in Fig. 6.5 (c), attests to the fact that there is no homogeneous bonding in the perfect crystal, as expected. For the amorphous structure, one can see a small contribution of homogeneous bonding, with N-N bonds around $1.48 \AA$ and B-B bonds at a slightly larger typical bond length, however this is truely a small fraction of the entire sample. Compared to the crystal structure, the majority bonding in the amorphous network remains heterogeneous, however the typical bond lengths are distorted, as indicated by the broadening of the peaks in the radial distribution function.

The bond angle distribution function (BDF), which defines the probability $g(\theta)$ of finding an atom with two bonds at an angle $\theta$, is plotted in Figs. 6.5 (e) and $6.5(\mathrm{f})$, for the crystal and amorphous structure, respectively. The notation $\angle \mathrm{X}-\mathrm{Y}$, is defined as an angle formed by two bonds where the atom at the vertex is of type $\mathrm{X}$ and the atoms it is bound to are of type $\mathrm{Y}$. The types $\mathrm{X}$ and $\mathrm{Y}$ can each be a set of one or more elements. The BDF of the crystal structure is defined by a single peak at $120^{\circ}$ which is directly determined by the hexagonal symmetry of the hexagonal boron nitride lattice. Due to atomic vibrations, induced by the thermal effects of a finite temperature, which slightly displace atoms from their ideal equilibrium lattice positions, the distribution is not a delta function at this angle, but rather a slightly broadened distribution. The distribution of bond angles between all atoms, as found in the amorphous network, is significantly broader, but is still centered around an angle of $120^{\circ}$, characteristic of trigonal bonding. The two satellite peaks centered around a bond angle of $80^{\circ}$ and $100^{\circ}$ approximately, correspond to bond angles formed by heterogeneous bonds with a boron $(\angle \mathrm{B}-\mathrm{N})$ or nitrogen $(\angle \mathrm{N}-\mathrm{B})$ atom at the vertex, respectively, as is visualized by the solid green and blue line in Fig. 6.5 (f). In summary, the loss of long range order due to the amorphization of a crystal lattice, can significantly broaden spectral features in the x-ray absorption spectroscopy, however the overall spectral character will still be determined by the local chemistry and structural order of the element that is being excited.

To determine whether amorphous boron phosphide phases could explain the broad absorption onset observed in the experimental B $K$-edge spectra collected for $c$-BP samples, an amorphous BP network $(a$-BP) was simulated in similar fashion as the 
amorphous boron nitride structure, described previously. A $c$-BP supercell was melted to create a liquid, which was subsequently quenched, resulting in an amorphous structure, a snapshot of which is visualized in Fig. 6.6(c). In stark contrast with boron nitride, in the boron phosphide melt and its derived quenched amorphous configurations, a strong segregation of boron and phosphorous atoms was observed. Almost instantaneously following the nucleation point of the melting process, boron atoms were observed to group together, leading to separate boron and phosphorous clusters. This observation can be quantified by the average RDF and BDF for the amorphous boron phosphide trajectory equilibrated at room temperature, as plotted in Figs. 6.7 (a) and 6.7 (b), respectively.

The computed radial distribution functions for the boron nitride structures, both crystalline and amorphous as shown in Figs. 6.5 (c) and 6.5 (d), showed a first-nearest neighbor peak that was clearly defined by a single Gaussian distribution, indicating that all atomic bonds belonged to a single population type and had similar character. Contrastingly, the first-nearest neighbor peak in the RDF computed for the amorphous boron phosphide trajectory, clearly comprises at least three distinct populations of bond lengths, as evidenced by the solid black line in Fig. 6.7 (a). The position of the highest peak out of the three overlaps with the position of the RDF for the perfect $c$-BP crystal thermally equilibrated at room temperature, represented by the dashed black line, indicating that there is still a significant population of boron-phosphorus bonds with a bond length comparable to the one found in the cubic crystal. The deconvoluted distribution, represented partly by the green (orange) solid curve in the same figure, shows that the shorter (longer) bond lengths can be attributed to homogeneous boron (phosphorous) bonds. This is a direct result of the earlier described segregation of boron and phosphorous atoms in separate clusters, directly following the melting of the $c$-BP crystal.

A similar effect can be seen in the bond angle distribution function computed for the amorphous boron phosphide structure, which is plotted in Fig. 6.7 (b). The BDF of the $a$-BP structure still has a majority contribution around an angle of $109^{\circ}$, which is characteristic of tetragonal bonding, as evidenced by the BDF computed for the perfect $c$-BP crystal, represented by the dashed black line. However, the bond angle distribution for $a$-BP is considerably broadened and a new feature at $60^{\circ}$ appears which does not correspond to tetragonal bonding found in $c$-BP. The deconvolution of the $\mathrm{BDF}$ in the distribution function of homogeneously bonded atoms, reveals that the contribution at $60^{\circ}$ can be attributed to homogeneous boron bonding, as evidenced by the solid green line. This is again due to the presence of local boron clusters in the amorphous boron phosphide structure, in which the boron atoms predominantly form rings amongst each other in the shape of equilateral triangles.

Quantifying the sizes and distributions of rings formed in amorphous networks is another useful measure to describe non-crystalline systems. We have employed the 


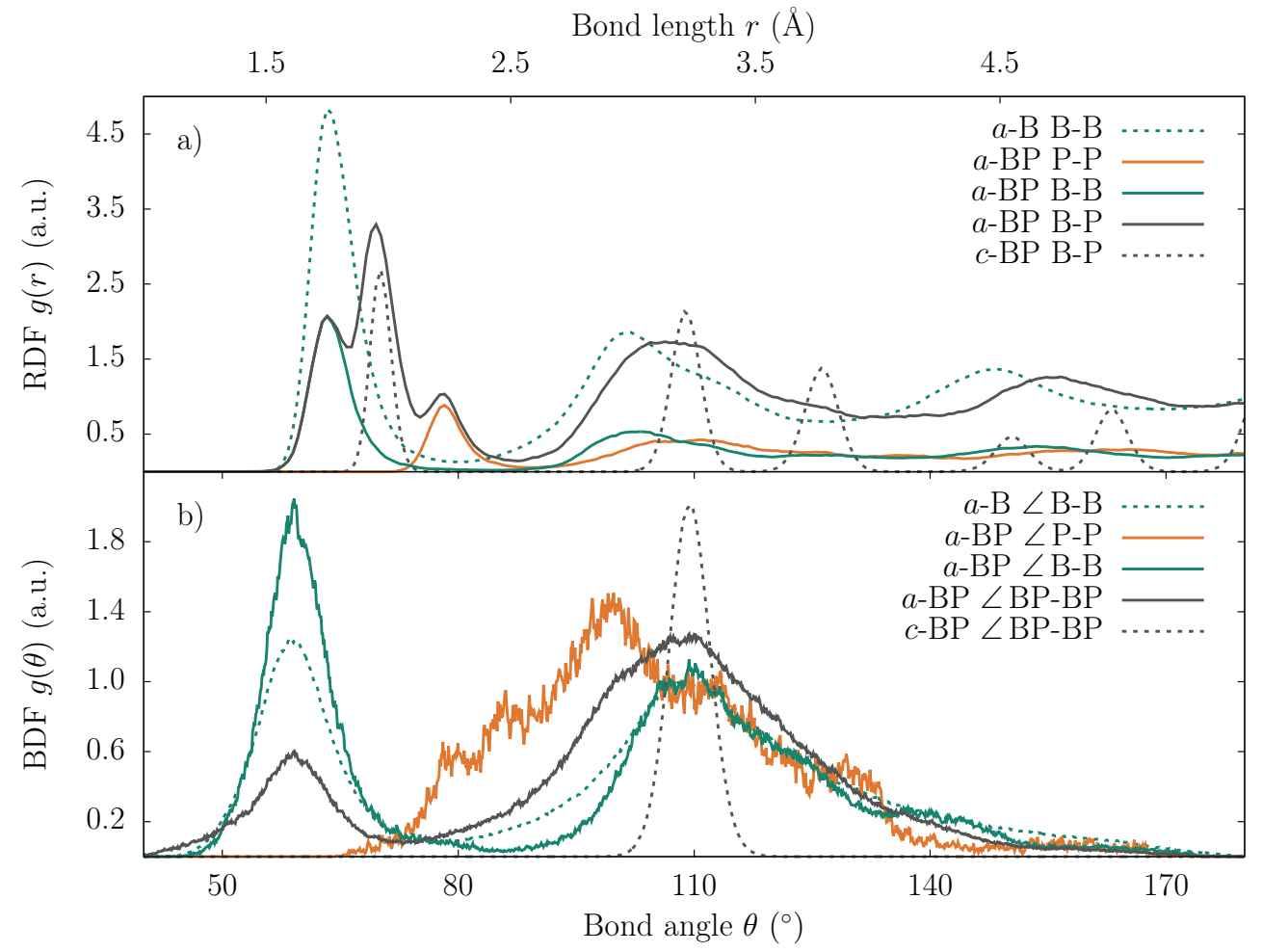

Figure 6.7: The (a) radial distribution an (b) bond angle distribution function for the simulated amorphous boron phosphide network, calculated as an average over 9000 snapshots from the trajectory of the structure equilibrated at room temperature. The solid black lines correspond to bonds and bond angles between all boron and phosphorus atoms, whereas the solid orange and green line only consider homogenous bonds between phosphorous and boron atoms, respectively. The dashed green and black lines in the top (bottom) panel corresponds to the computed RDF (BDF) of an amorphous boron and cubic boron phosphide structure, and serve as a reference. 


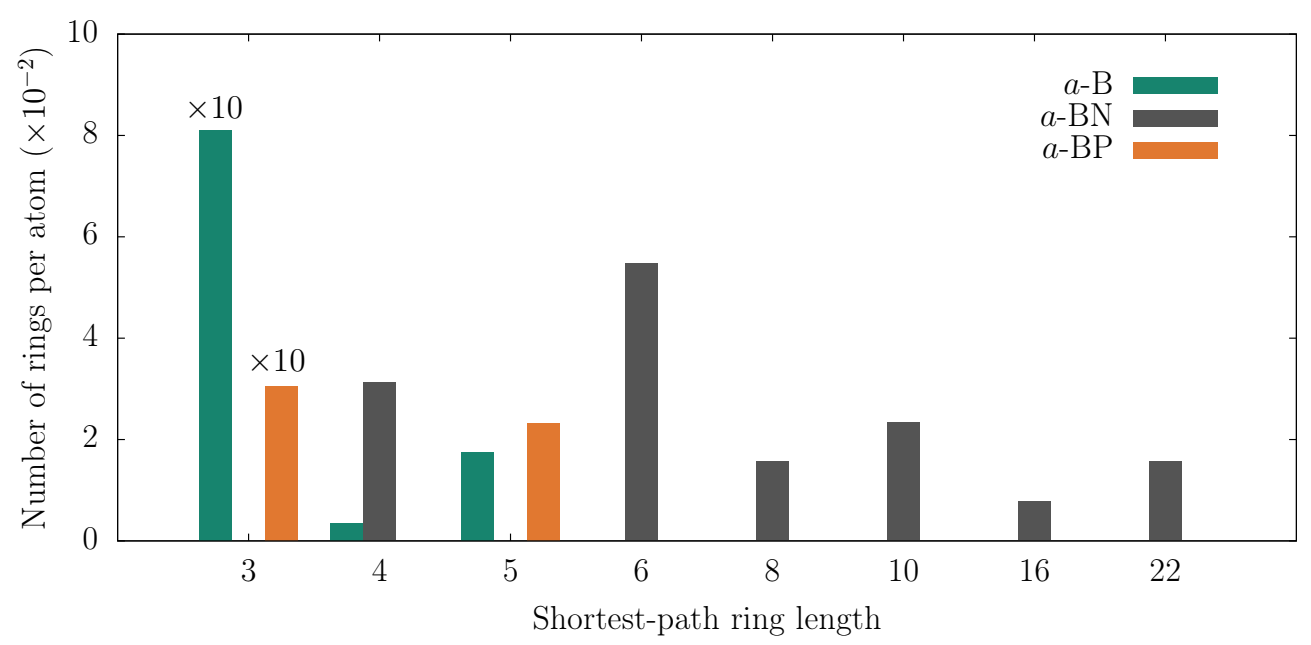

FIgURE 6.8: Shortest-ring path analysis performed on the equilibrated trajectories of the amorphous boron $(a-\mathrm{B})$, boron nitride $(a$-BN) and boron phosphide $(a$-BP $)$ networks. The horizontal axis defines the size of the ring, or number of atoms that make up the ring, and the vertical axis is the frequency of occurrence of that ring size. All frequencies are normalized to the number of atoms in the cell of the simulation. The frequencies for rings of size 3 have been scaled down by a factor of 10 to improve visual clarity.

shortest-path ring analysis as described by Franzblau[110], which finds all the shortestpath rings in a structural network and determines their ring length distribution. A ring is defined as any closed path formed by neighboring atoms at a pre-defined maximum distance. With this simple definition, rings of arbitrarily large size can be found in almost any network, reducing the descriptive value of the structural property, which is why the constraint of shortest-path is added to the definition. This addition requires that any path does not contain a shorter closed path within itself. With this definition, we have computed the averaged shortest-ring path distributions for the trajectories of the amorphous boron, boron nitride and boron phosphide systems, as shown in Fig. 6.8. The amorphous boron nitride network has shortest-path rings of varying lengths, but all of them contain an even number of atoms. This is a direct result of the predominantly heterogeneous bonding of this amorphous structure, as evidenced by the previously discussed RDF and BDF shown in Fig. 6.5. If there is no homogeneous bonding, then any shortest-path ring has to consist of an even numbers of atoms. The most populous ring type in amorphous boron nitride is made of 6 atoms, which corresponds directly to the hexagonal rings of the $h$-BN crystal structure which shows once more that, despite the lack of long range order, the amorphous network maintains a local structure that is reminiscent of the hexagonal crystal lattice. The amorphous boron network does not contain any rings longer than 5 atoms and rings formed by three boron atoms are numerous. These rings of 3 boron atoms take on 


\begin{tabular}{lccc}
\hline Phase & $N_{\text {atom }}$ cell $^{-1}$ & $E_{\text {tot }}$ & $\Delta H_{f}$ \\
\hline$\alpha$-B & 36 & -241.2 & -12.7 \\
$c$-BP & 8 & -51.7 & -10.7 \\
$h$-BP & 4 & -24.3 & -9.9 \\
\hline$h$-BN & 4 & -35.1 & -14.1 \\
$c$-BN & 8 & -70.0 & -9.5 \\
\hline
\end{tabular}

TABlE 6.2: Computed formation enthalpies for different boride crystal phases. The second column represents the number of atoms in the unit cell used in the calculation, $E_{\text {tot }}$ represents the total energy for the unit cell as obtained through DFT and $\Delta H_{f}$ is the derived formation enthalpy from its atomic constituents per formula unit. Both the total energy and formation enthalpy are given in $\mathrm{eV}$.

the shape of equilateral triangles, exactly as they are found in the icosahedral units of the icosahedral boron crystal structures. The shortest-path ring distribution for the amorphous boron phosphide network is highly comparable to that of the amorphous boron structure and confirms the similarities between the $a$-B and $a$-BP structures.

The similarity between $a$-B and $a$-BP networks, is corroborated by the RDF and BDF as shown in Figs. 6.7 (a) and 6.7 (b), where the dashed green line represents the computed RDF and BDF, respectively, for an amorphous boron structure equilibrated at room temperature. The characteristic bond length is centered around $1.76 \AA$ and the typical bond angles are $60^{\circ}$ and $107^{\circ}$, which correspond exactly to the dominant bond lengths and bond angles found in icosahedral borides. The typical bond lengths and angles of the B-B bonds in $a$-BP show a high degree of similarity with the characteristic values found in the simulated amorphous boron structure, so it is apt to label the boron clusters in the $a$-BP as an amorphous boron phase. This raises the question, why boron nitride maintains a predominantly heterogeneously and trigonally bonded structure when amorphisized by quenching from a melt, yet in boron phoshide, a phase separation is observed, where its atomic constituents group into elementally homogeneous clusters and the boron atoms seem to form structures characteristic of purely amorphous boron phases. To answer this question, one can approach the formation of the various boron compounds discussed so far, from a thermodynamic point of view.

\subsubsection{FORMATION ENTHALPIES OF BORON COMPOUNDS}

The stability and likelihood of formation of a certain crystal phase can be estimated by calculating the formation enthalpy. To assess the probability of hexagonal boron phosphide or amorphous boron phases growing in parallel with the intended cubic phase of the studied boron phosphide samples, we have computed the formation enthalpies of various boron and boron phosphide phases, as tabulated in Table 6.2. 
The formation enthalpy $\Delta H_{f}$ in Table 6.2 is given per formula unit and is computed as

$$
\Delta H_{f}=E_{\mathrm{tot}}-\sum_{i}^{N} n_{i} E_{i}
$$

where $E_{\text {tot }}$ is the total energy of the system and the sum iterates over all $N$ elemental types $X$ in the cell, where $n_{i}$ corresponds to the number of atoms of type $X$ in the cell and $E_{i}$ represents the total energy of an isolated atom of type $X$.

The lower the formation enthalpy, the more stable and the more likely the corresponding crystal phase is to form. The computed $\Delta H_{f}$ show that the cubic phase of boron phosphide is more stable compared to the hexagonal phase, which is in line with the successful synthesization of $c$-BP but the current lack of a pure $h$-BP synthesis process. We have also computed the formation enthalpy of the $\alpha$-rhombohedral phase of elemental boron, labeled $\alpha$-B in Table 6.2, which is energetically more favorable to form than both boron phosphide phases. This result is in line with the observation described in the previous section, that upon melting of boron phosphide, the boron and phosphorus atoms tend to segregate into homogeneous clusters, where the boron atoms form structures with structural properties characterstic for icosahedral boron phases. Consequently, this suggests that in a boron phosphide growing process, there is a reasonable chance of the formation of elemental boron phases. Note that contrastingly, hexagonal boron nitride is energetically more favorable than the elemental boride structure, as was already suggested by the lack of homogeneous bonding found in both the experimental and theoretical analyses of amorphous boron nitride. We now pose the question, whether the presence of amorphous boron phases in the experimentally produced $c$-BP samples, could explain the broad absorption onset observed in the experimental boron $K$-edge absorption spectrum.

\subsubsection{X-RAY ABSORPTION SPECTROSCOPY OF AMORPHOUS BORON}

We have collected the B $K$-edge for an amorphous boron sample, deposited with electron beam evaporated physical vapor deposition, represented by the solid green line in Fig. 6.9. Details of the deposition can be found in Section 6.2.2. The solid black line in the same figure is the calculated spectrum for the simulated amorphous boron structure and the dashed green line references the experimentally collected spectrum for the $c$-BP sample S1.

For the amorphous boron structures, both experiment and theory find a broad and smooth spectrum without any distinctive narrow spectral features. The dashed line labeled $C$, marks the position of a spectral feature present in the experimental spectrum at $194 \mathrm{eV}$, due to the boron oxide $\mathrm{B}_{2} \mathrm{O}_{3}$ phase[1], which is present on the sample surface due to exposure to atmospheric conditions after sample deposition. Most interestingly, both the simulated and experimental spectrum, show a broad and gradual onset of 


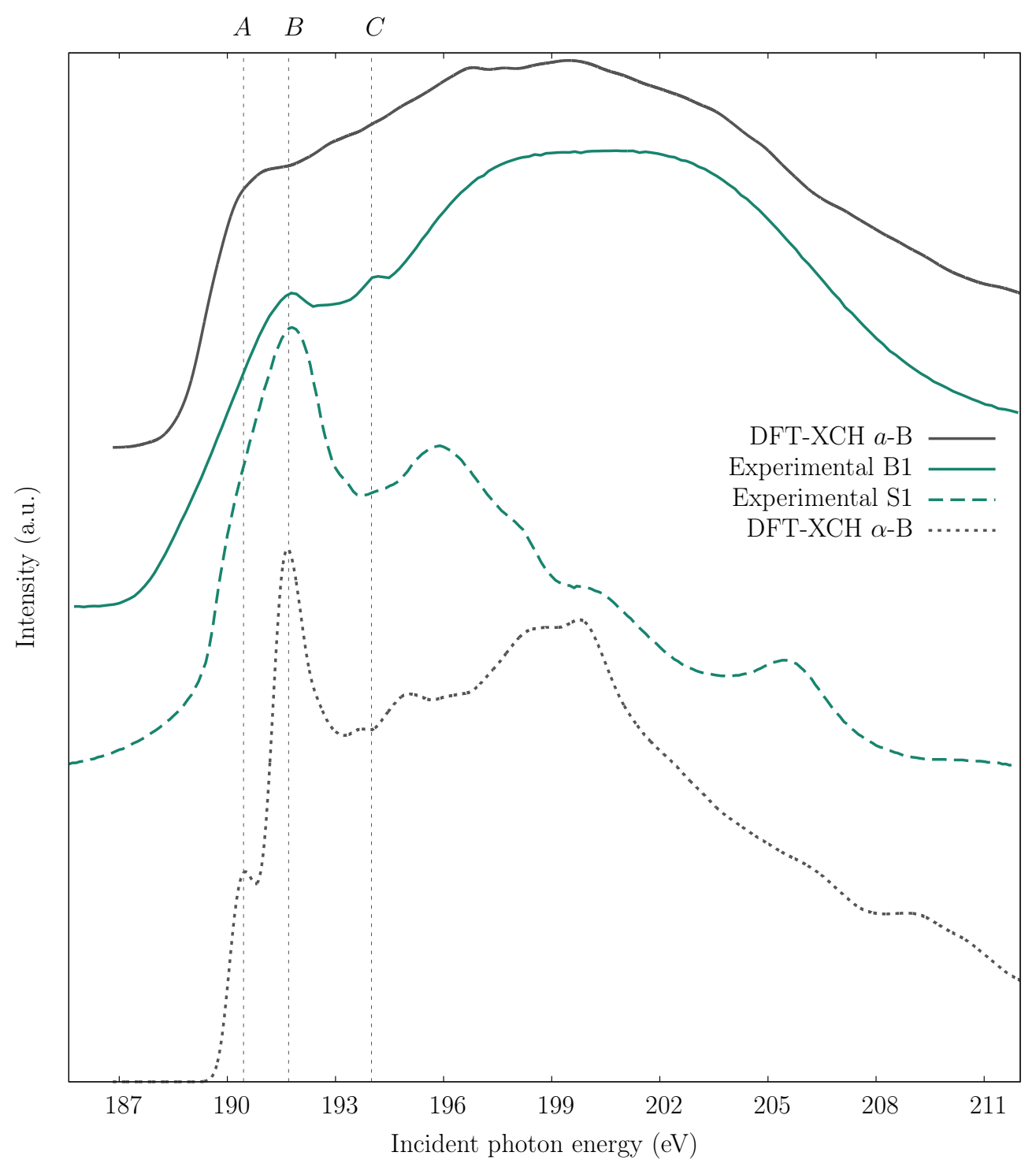

FiguRE 6.9: Experimentally collected x-ray absorption spectra of an amorphous boron (B1) and cubic boron phosphide (S1) sample, represented by a solid and dashed green line, respectively. The solid and dashed black lines represent the simulated B $K$-edge spectrum for the amorphous boron structure and the thermally equilibrated $\alpha$-rhombohedral boron crystal structure, respectively. The spectra have been translated vertically in order to improve visual clarity. 
the absorption edge, reminiscent of what has been observed for the $c$-BP samples investigated in this work, shown as a dashed green line in Fig. 6.9. To show that the broad onset is indeed caused by the amorphous character of the boride, we have also simulated the boron $K$-edge for the most simple allotrope of elemental boron, $\alpha$-rhombohedral boron $(\alpha-\mathrm{B})$, which is represented by the dashed black line in Fig. 6.9. The most prominent feature in the $\alpha$-rhombohedral spectrum, indicated by the dashed line labeled $B$, by definition associated with icosahedral boron atoms, is also still present in the amorphous boron spectrum, which proves that there are boron atoms present in the amorphous structure with an icosahedral configuration. However, the spectrum for the rhombohedral boron crystal structure clearly has a sharper absorption onset and is significantly blue shifted compared to the other spectra. This shows that it is not the crystalline but the amorphous character of the boron structure, that is responsible for the broad and gradual absorpion onset. This gives reasonable cause to believe that the broad absorption edge, as observed for $c$-BP, is due to the presence of amorphous boron phases in the studied samples.

Moreover, this hypothesis concerns a structural model that, containing exclusively boron atoms, strictly only influences the spectrum for excitations of $1 s$ electrons from boron atoms (i.e. the boron $K$-edge). By adding amorphous boron phases, to the initial purely cubic boron phosphide crystalline structural model of the $c$-BP samples, only the boron $K$-edge spectroscopy is affected and the phosphorus $L_{2,3}$ spectrum remains unchanged. The hypothesis of the presence of amorphous boron phases in the $c$-BP samples, therefore also explains the juxtaposition of the ability of the cubic boron phosphide structure to successfully model the $\mathrm{P} L_{2,3}$-edge and its simultaneous inability to accurately reproduce the B $K$-edge. This hypothesis indirectly implies that the boron to phosphorus ratio in the samples has to be off-stoichiometric, with an excess of boron.

\subsection{CONCLUSIONS}

We have presented experimentally collected x-ray absorption near-edge spectroscopy measurements at the boron $K$-edge and phosphorus $L_{2,3}$-edge for cubic boron phosphide samples, grown with chemical vapor deposition. Simulated x-ray absorption spectra from first principles show that the $\mathrm{P} L_{2,3}$-edge is aptly described by a simple $c$-BP crystal structural model, however the same model fails to reproduce the broad absorption onset observed in the B $K$-edge. Intrinsic crystal point defects and the hexagonal boron phosphide phase are discussed as a potential explanation, however calculations show that the corresponding spectroscopic signatures, or a superposition of a combination of these structures are too narrow to be able to reproduce the smooth and broad character of the experimental B $K$-edge. 
Broad spectral features can arise due to the amorphization of crystalline phases, which was illustrated by means of experimental and theoretical x-ray absorption spectra for hexagonal and amorphous boron nitride structures. Ab initio molecular dynamics simulations were employed to model amorphous boron phosphide structures, which revealed that upon the melting of a BP crystal, boron and phosphorous atoms segregated in predominantly homogeneously bonded clusters. This effect was explained by the formation enthalpies of the various boride phases, which showed that the formation of an elemental boron phase is energetically more favorable compared to the cubic boron phosphide phase. This leads to the conclusion that during the $c$ - BP growth process there is a significant probability of the formation of amorphous boron phases. The boron clusters that formed in the simulated amorphous boron phosphide structure, were shown to adopt a structure commonly found in icosahedral and amorphous boron structures. Measured and simulated x-ray absorption spectroscopy of amorphous boron structures showed a broad absorption onset in their B $K$-edge, directly comparable to that observed in the spectra obtained for the $c$-BP samples. These results show that the broad absorption onset, measured in the B $K$-edge of the CVD grown $c$-BP samples, can be attributed to amorphous boron phases, which provides strong evidence for the presence of elemental boron clusters in the $c$-BP samples.

\section{ACKNOWLEDGMENTS}

This work is supported by NanoNextNL, a micro and nanotechnology programme of the Dutch Government and 130 partners. We acknowledge the support of the Center for X-ray Optics of Lawrence Berkeley Laboratory and the Industrial Focus Group XUV Optics at the MESA+ Institute for Nanotechnology at the University of Twente, notably the partners ASML, Carl Zeiss SMT GmbH, and the Foundation FOM. All the computational work was performed at the Molecular Foundry which is supported by the Office of Science, Office of Basic Energy Sciences, of the United States Department of Energy under Contract No. DE-AC02-05CH11231. 


\section{REFERENCES}

[1] S. P. Huber, E. Gullikson, R. W. E. van de Kruijs, F. Bijkerk, and

D. Prendergast, Phys. Rev. B 92 (2015) 10.1103/physrevb.92.245310.

[3] S. P. Huber, E. Gullikson, C. D. Frye, J. H. Edgar, R. W. E. van de Kruijs, F. Bijkerk, and D. Prendergast, Submitted to Chemistry of Materials.

[22] V. Medvedev, A. Yakshin, R. van de Kruijs, and F. Bijkerk, Optical Materials Express 5, 1450 (2015).

[23] B. Padavala, C. D. Frye, X. Wang, Z. Ding, R. Chen, M. Dudley, B. Raghothamachar, P. Lu, B. N. Flanders, and J. H. Edgar, Crystal Growth \& Design 16, 981 (2016).

[24] B. Padavala, C. Frye, X. Wang, B. Raghothamachar, and J. Edgar, Journal of Crystal Growth (2016) 10.1016/j.jcrysgro.2016.05.031.

[36] D. Prendergast, and G. Galli, Phys. Rev. Lett. 96, 215502 (2006).

[42] J. Underwood, and E. Gullikson, Journal of Electron Spectroscopy and Related Phenomena 92, 265 (1998).

[43] E. M. Gullikson, S. Mrowka, and B. B. Kaufmann, Recent developments in euv reflectometry at the advanced light source, 2001.

[47] G. Kresse, and J. Furthmüller, Physical Review B 54, 11169 (1996).

[49] E. L. Shirley, Phys. Rev. B 54, 16464 (1996).

[57] I. Jiménez, A. F. Jankowski, L. J. Terminello, D. G. J. Sutherland, J. A. Carlisle, G. L. Doll, W. M. Tong, D. K. Shuh, and F. J. Himpsel, Phys. Rev. B 55, 12025 (1997).

[58] M. Niibe, K. Miyamoto, T. Mitamura, and K. Mochiji, Journal of Vacuum Science \& Technology A 28, 1157 (2010).

[59] I. Caretti, and I. Jiménez, Journal of Applied Physics 110, 023511, (2011). 
[60] K. Simonov, N. Vinogradov, M. Ng, A. Vinogradov, N. Mårtensson, and

A. Preobrajenski, Surface Science 606, 564 (2012).

[61] P. E. Blöchl, Physical Review B 50, 17953 (1994).

[62] G. Kresse, and D. Joubert, Physical Review B 59, 1758 (1999).

[63] J. P. Perdew, K. Burke, and M. Ernzerhof, Phys. Rev. Lett. 77, 3865 (1996).

[64] A. Tkatchenko, and M. Scheffler, Phys. Rev. Lett. 102, 073005 (2009).

[67] A. H. England, A. M. Duffin, C. P. Schwartz, J. S. Uejio, D. Prendergast, and R. J. Saykally, Chemical Physics Letters 514, 187 (2011).

[92] B. Padavala, C. Frye, Z. Ding, R. Chen, M. Dudley, B. Raghothamachar, N. Khan, and J. Edgar, Solid State Sciences 47, 55 (2015).

[93] G. Li, J. K. Abbott, J. D. Brasfield, P. Liu, A. Dale, G. Duscher, P. D. Rack, and C. S. Feigerle, Applied Surface Science 327, 7 (2015).

[94] Z. Jia, J. Zhu, C. Jiang, W. Shen, J. Han, and R. Chen, Applied Surface Science 258, 356 (2011).

[95] W. Liu, H. Tu, H. Yang, S. Zhang, L. Yan, C. Huo, and X. Su, Journal of Alloys and Compounds 538, 169 (2012).

[96] V. A. Mukhanov, P. S. Sokolov, Y. L. Godec, and V. L. Solozhenko, J. Superhard Mater. 35, 415 (2013).

[97] V. A. Mukhanov, P. S. Sokolov, O. Brinza, D. Vrel, and V. L. Solozhenko, J. Superhard Mater. 36, 18 (2014).

[98] H. Sugimoto, M. Fujii, and K. Imakita, RSC Adv. 5, 8427 (2015).

[99] V. A. Mukhanov, D. Vrel, P. S. Sokolov, Y. L. Godec, and V. L. Solozhenko, Dalton Trans. (2016) 10.1039/c6dt00435k.

[100] A. López-Castillo, Int. J. Quantum Chem. 112, 3152 (2012).

[101] J. Yu, and W. Guo, Appl. Phys. Lett. 106, 043107 (2015).

[102] D. Çakır, D. Kecik, H. Sahin, E. Durgun, and F. M. Peeters, Phys. Chem. Chem. Phys. 17, 13013 (2015).

[103] J. Dong, H. Li, and L. Li, NPG Asia Materials 5, e56 (2013).

[104] M. Mirzaei, and M. Meskinfam, Solid State Sciences 13, 1926 (2011).

[105] Z. Sohbatzadeh, M. Roknabadi, N. Shahtahmasebi, and M. Behdani, Physica E: Low-dimensional Systems and Nanostructures 65, 61 (2015).

[106] S.-f. Wang, and X.-j. Wu, Chin. J. Chem. Phys. 28, 588 (2015).

[107] D. Vanderbilt, Phys. Rev. B 41, 7892 (1990).

[108] K. Laasonen, R. Car, C. Lee, and D. Vanderbilt, Phys. Rev. B 43, 6796 (1991).

[109] D. McCulloch, D. Lau, R. Nicholls, and J. Perkins, Micron 43, 43 (2012). 
[110] D. S. Franzblau, Phys. Rev. B 44, 4925 (1991). 

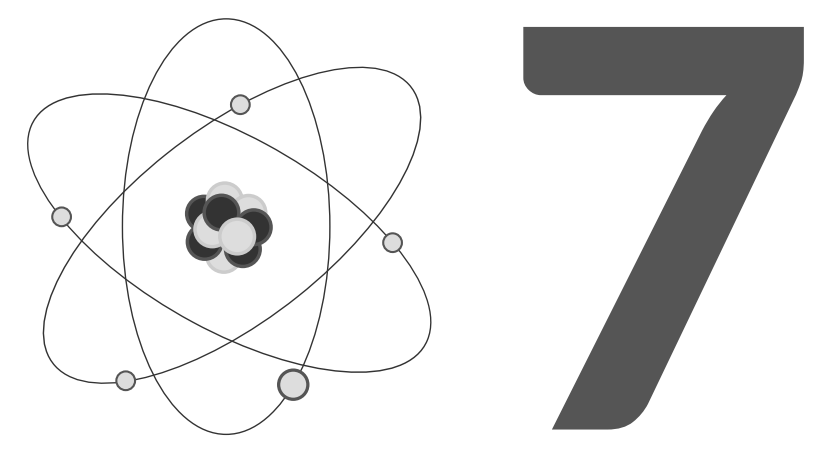

VALORIZATION 


\section{ABSTRACT}

The research described in this work has relied heavily on a single piece of software to facilitate the simulation of x-ray absorption spetroscopy. This code package, named ShirleyXAS, has been designed and is still actively developed by the Prendergast group at the Molecular Foundry user facility, which is part of Lawrence Berkeley National Laboratory. ShirleyXAS is a typical piece of academic software; intrinsically powerful with a large potential for solving interesting and relevant questions in both fundamental research and applied environments, but fundamentally inaccessible to non-experts and only efficiently operable after extensive training with a steep learning curve.

The Molecular Foundry is a nanoscience research facility that aims to provide users from a wide range of disciplines with access to cutting-edge experimental instrumentation and theoretical expertise. Providing users with a direct access to this theoretical expertise, without having to rely on the experts to operate ShirleyXAS, would yield a significant increase in independence and efficieny for the users. To this end, a graphical user interface (GUI) was designed and developed, that provides an instinctive environment for the user to perform x-ray absorption spectroscopy calculations on the highly optimized computing resources of the Molecular Foundry. The interface has been built as a browser based application, allowing this webtool, dubbed WEBXS, to provide easy access from any location with an internet connection. In this valorization chapter, the challenges faced in designing and building WEBXS will be described, which may prove useful for other scientific research groups that wish to make their own academic codes available to a wider audience. The chapter will be concluded with a list of recent publications that have partly been made possible through the use of WEBXS for their $\mathrm{x}$-ray absorption spectroscopy simulations and analysis. 


\subsection{GOALS AND SPECIFICATIONS}

The original goals envisioned for WEBXS were defined by two key descriptions: userfriendliness and deployability through configurability. The first keyword, user-friendliness, is obviously aimed at the end user of the webtool. The interface provided by the webtool should be easily accessible and intuitive, in order for users to efficiently perform complicated x-ray absorption calculations from first principles without any specific training. WEBXS has been built as a browser based application, which can in principle satisfy both conditions. Internet connections and browsers nowadays are ubiquitous, making the webtool easily available to any user without having to download, compile and or install a dedicated application. Additionally, due to the widespread use of browser based applications, many programming toolkits exist to aid the development of graphical user interfaces, and the resulting interfaces will be familiar to a significant portion of users. Designing user-friendly interfaces is a study in itself and is certainly not the author's strong suit nor specialization and therefore, outside the global considerations described up till this point, did not receive any further focused attention.

The second key description of the design goals of WEBXS is deployability through configurability. The goal of the webtool is to facilitate access for its end users to the computational resources that are made available to them, which are often massively parallelized clusters of supercomputers designed for high throughput and computationally intensive software. In general, a user can have access to many different computing resources, each one of which can be made up of yet more individual computing systems. These computational systems can have a wide range of different architectures and available platforms, to which the webtool needs to be adapted in order to function properly. Rewriting the webtool for each individual computing resource as they become available is untenable and therefore it needed to be designed in such a way, that it could be easily transferred and deployed to a new system, by a minimal amount of configuration.

\subsection{GLOBAL ARCHITECTURE AND DESIGN}

To explain the global architecture and design of the WEBXS webtool, the computing resources of the National Energy Research Scientific Computing Center (NERSC), on which WEBXS has been deployed and has been used actively by users of the Molecular Foundry, will be used as an example. During the development of WEBXS, it has been deployed on three NERSC computing clusters named Hopper, Edison and Cori. A schematic overview of this situation is shown in Fig. 7.1. The WEBXS webtool provides a graphical user interface which presents data to the user and allows the user to submit new calculations to a computing cluster. The communication of the webtool with the computing cluster is facilitated through some communication protocol which will be described later. 


\section{NERSC}

\section{WebXS}

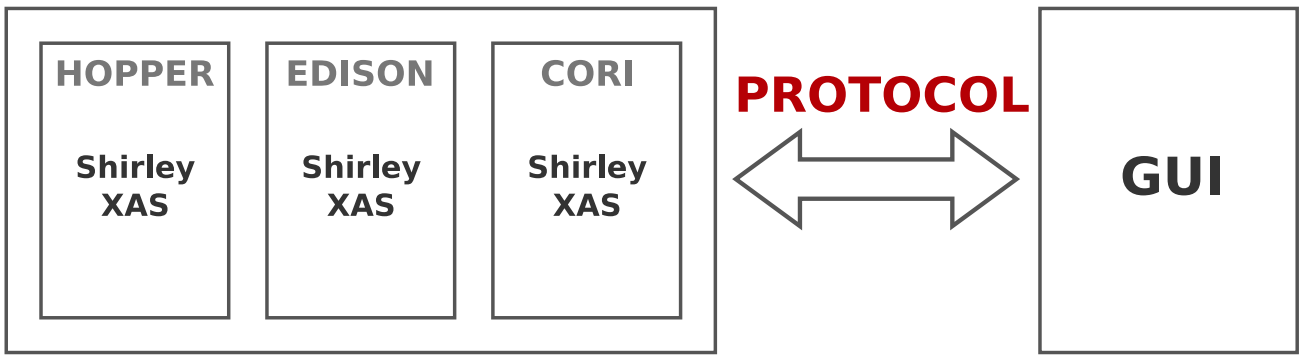

Figure 7.1: A schematic overview of the interaction between WeBXS and in this example the computing resources at NERSC as facilitated by the NERSC web toolkit (NEWT). Hopper, Edison and Cori are the names of the computing machines at NERSC on which ShirleyXAS calculations were ran during the development of WeBXS. WeBXS can communicate with the NERSC machines over a communication protocol that will be specified later.

\subsubsection{DECOUPLING COMPUTATION AND DATA PRESENTATION}

To run any code (in this case ShirleyXAS) on a big parallel computational cluster, input files will have to be generated that define the locations of the required binaries, as well as batch scripts that will be submitted to the scheduler, that will allow the code to be run on multiple processors. The locations of the required binaries and the scheduler most likely will change from machine to machine, which can drastically change how these input files have to be formatted. However, regardless of how a calculation should be run on any specific machine, the eventual presentation of the data to the user in the web interface should be completely independent and agnostic of these specifics. This realization brings forth the most important and infuential design choice of the whole webtool, which is to separate the WEBXS webtool into two parts; the webportal and the webservice. This situation is drawn schematically in Fig. 7.2, to indicate how the different parts of the WEBXS webtool would interact with one another and with the specific cluster that it is deployed on.

When a user wants to submit a calculation, the webportal collects the relevant input parameters provided by the user in the interface and submits a request through the communication protocol directly to the webservice. The webservice, configured for the machine that it is deployed on, will transform the input parameters into the input files that the ShirleyXAS code requires, and will submit the calculation to the scheduler of the cluster. Similarly, the webportal can make a request to the webservice to retrieve the status of submitted calculations as well as retrieve data from completed calculations. The webportal will translate the received data and visualize it in the web interface for the end user.

With this decoupled setup, the WEBXS can be deployed on any computational resource 


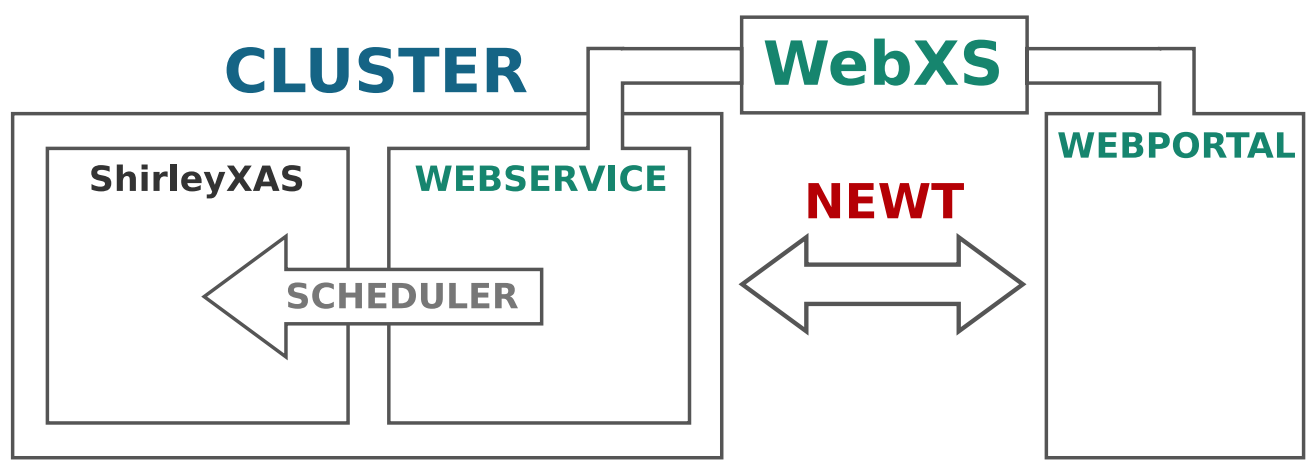

Figure 7.2: A schematic overview of the split of WEBXS into the webportal and the webservice. The webservice needs to be deployed on the cluster to have direct access to the scheduler in order to submit calculations. The webportal does not necessarily have to be on a server that has direct access to the filesystem of the cluster. The webportal will communicate with the webservice through the communication protocol (NEWT).

and the only part that has to be adapted is the interaction between the webservice and the cluster. In addition to this significant benefit, there is another important argument to separate the webservice and the webportal. If the webtool were to be one component, the web interface with which the user interacts, must by definition have direct access to the filesystem of the cluster, which can pose a security threat. For this reason, computational resources often do not even allow web interfaces to be served from the actual cluster but have to be ran on an isolated server, in which case the webtool hás to be separated into two components.

\subsubsection{COMMUNICATION PROTOCOL}

To allow the webportal to communicate with the webservice, a communication protocol is required, which NERSC provides in the form of an application programming interface (API) called NEWT. NEWT is developed by NERSC and specifically designed to work in conjunction with the NERSC computing resources. It cannot be assumed that identical functionality will be available at any other computing resource where WEBXS might be deployed. To maintain the configurability and deployability, the assumptions about the communication protocol that the webtool makes, have to be abstracted to the bare minimum.

A minimum requirement for any communication protocol that would make the webtool possible, is the ability to issue a command on the operating system of the target computing resource. Therefore, we restrict any interaction between the webportal and webservice to be defined as a direct call to a top level script of the webservice. This 
maximizes the probability that the required functionality will be implemented by the available communication protocol.

Similar considerations have to be made for the format of any data that has to accompany calls from the webportal to the webservice. To maximize compatibility with any feasible communication protocol, we have standardized the format of data communication between the webportal and the webservice according to the JavaScript Object Notation (JSON). This lightweight data-interchange format is based on a subset of the JavaScript programming language, which is a language that is predominantly used to create user interfaces in browsers, as is the webportal of WEBXS. Additionally, the usage of JSON is ubiquitous and heavily standardized and many programming languages provide either native integration or mature libraries to parse and write JSON data.

\subsection{CONCLUSIONS}

In this valorization chapter, I have described the process of designing the web based application WEBXS, that provides users of the Molecular Foundry user facility easy and direct access to the academic software ShirleyXAS, which has been developed and used heavily in this work. User interfaces such as WEBXS, enable non-experts to directly tap from the power that academic codes often possess but is typically hard to harvest without specific training or specialized skills. During the design, I focused on creating an architecture that would make the webtool easily deployable and compatible with a wide range of computational environments that are often encountered in scientific computing. This would ensure that the webtool could be used for all the computing resources a user has access to, without having to rewrite the code everytime.

The most significant design choices, which can be useful and applied to any piece of academic software that aims to provide a web interface to computational resources, can be summarized in two points

- Separate computation and data representation functionality

- Restrict the method and data format of communication

The part of the webtool that serves as an interface between the user input and the cluster, depends heavily on the specific computational resource that it is deployed on. However, the data presentation in the graphical user interface should not be dependent on these specifics, and therefore this part of the webtool should be decoupled. This decoupling forces the introduction of a communication protocol that facilitates the communication between the webportal and webservice, which leads to the second point.

Since the specific implementation of the communication protocol available at a particular computing resource is not always controllable, assumptions about its functionality 
and/or interface have to be minimized to maintain deployability. To achieve this, WEBXS restricts communication between the webportal and the webservice to be unidirectional calls from the webportal to scripts of the webservice, where all necessary data is transmitted as a single JSON serialized string. Following these two design principles, WEBXS can be deployed on almost any computational resource with minimal efforts, by simply adapting a single configuration file for the webportal and the webservice.

The WEBXS webtool has been actively used by several users of the Molecular Foundry over the last two years and in that capacity it has contributed to scientific work that has led to multiple publications. A short list of publications that have benefitted from the use of ShirleyXAS through the WEBXS webtool is presented below.

\section{PUBLICATIONS PARTLY MADE POSSIBLE BY WEBXS}

[111] A. B. Altman, C. D. Pemmaraju, C. Camp, J. Arnold, S. G. Minasian, D. Prendergast, D. K. Shuh, and T. Tyliszczak, "Theory and x-ray absorption spectroscopy for aluminum coordination complexes - Al $K$-edge studies of charge and bonding in (BDI) $\mathrm{Al},(\mathrm{BDI}) \mathrm{AlR}_{2}$, and (BDI) $\mathrm{AlX}_{2}$ complexes", J. Am. Chem. Soc. 137, 10304 (2015).

[112] W. S. Drisdell, R. Poloni, T. M. McDonald, T. A. Pascal, L. F. Wan, C. D. Pemmaraju, B. Vlaisavljevich, S. O. Odoh, J. B. Neaton, J. R. Long, D. Prendergast, and J. B. Kortright, "Probing the mechanism of $\mathrm{CO}_{2}$ capture in diamine-appended metal-organic frameworks using measured and simulated x-ray spectroscopy", Phys. Chem. Chem. Phys. 17, 21448 (2015).

[113] T. A. Pascal, C. D. Pemmaraju, and D. Prendergast, "X-ray spectroscopy as a probe for lithium polysulfide radicals", Phys. Chem. Chem. Phys. 17, 7743 (2015).

[114] S. N. Patel, G. M. Su, C. Luo, M. Wang, L. A. Perez, D. A. Fischer, D. Prendergast, G. C. Bazan, A. J. Heeger, M. L. Chabinyc, and E. J. Kramer, "NEXAFS spectroscopy reveals the molecular orientation in blade-coated pyridal[2,1,3]thiadiazole-containing conjugated polymer thin films", Macromolecules 48, 6606 (2015).

[115] C. P. Schwartz, and D. Prendergast, "Communication: on the difficulty of reproducibly measuring $\mathrm{PbCl}_{2}$ x-ray absorption spectra", The Journal of Chemical Physics 143, 111102 (2015). 
[116] C. Schwartz, D. Nordlund, T.-C. Weng, D. Sokaras, L. Mansfield, A. S. Krishnapriyan, K. Ramanathan, K. E. Hurst, D. Prendergast, and S. T. Christensen, "Electronic structure study of the CdS buffer layer in CIGS solar cells by x-ray absorption spectroscopy: experiment and theory", Solar Energy Materials and Solar Cells 149, 275 (2016).

[117] J. W. Smith, R. K. Lam, O. Shih, A. M. Rizzuto, D. Prendergast, and R. J. Saykally,

"Properties of aqueous nitrate and nitrite from x-ray absorption spectroscopy", The Journal of Chemical Physics 143, 084503 (2015).

[118] L. F. Wan, J. Wright, B. R. Perdue, T. T. Fister, S. Kim, C. A. Apblett, and D. Prendergast, "Revealing electronic structure changes in chevrel phase cathodes upon mg insertion using x-ray absorption spectroscopy", Phys. Chem. Chem. Phys. 18, 17326 (2016). 


\section{BIBLIOGRAPHY}

[1] S. P. Huber, E. Gullikson, R. W. E. van de Kruijs, F. Bijkerk, and

D. Prendergast, Phys. Rev. B 92 (2015) 10.1103/physrevb.92.245310.

[2] S. P. Huber, E. Gullikson, J. Meyer-Ilse, C. D. Frye, J. H. Edgar, R. W. E. van de Kruijs, F. Bijkerk, and D. Prendergast, Submitted to Chemistry of Materials.

[3] S. P. Huber, E. Gullikson, C. D. Frye, J. H. Edgar, R. W. E. van de Kruijs, F. Bijkerk, and D. Prendergast, Submitted to Chemistry of Materials.

[4] S. P. Huber, V. V. Medvedev, E. Gullikson, B. Padavala, J. H. Edgar, R. W. E. van de Kruijs, F. Bijkerk, and D. Prendergast, Submitted to Phys. Rev. B.

[5] S. P. Huber, R. W. E. van de Kruijs, A. E. Yakshin, E. Zoethout, K.-J. Boller, and F. Bijkerk, Opt. Express 22, 490 (2014).

[6] T. Ogitsu, F. Gygi, J. Reed, Y. Motome, E. Schwegler, and G. Galli, J. Am. Chem. Soc. 131, 1903 (2009).

[7] K. S. Novoselov, Science 306, 666 (2004).

[8] C. Zhi, Y. Bando, C. Tang, H. Kuwahara, and D. Golberg, Adv. Mater. 21, 2889 (2009).

[9] L. Song, L. Ci, H. Lu, P. B. Sorokin, C. Jin, J. Ni, A. G. Kvashnin, D. G. Kvashnin, J. Lou, B. I. Yakobson, and P. M. Ajayan, Nano Letters 10, 3209 (2010).

[10] Y. Chen, J. Zou, S. J. Campbell, and G. Le Caer, Applied Physics Letters 84, 2430 (2004).

[11] A. Zunger, A. Katzir, and A. Halperin, Phys. Rev. B 13, 5560 (1976). 
[12] K. K. Kim, A. Hsu, X. Jia, S. M. Kim, Y. Shi, M. Hofmann, D. Nezich, J. F. Rodriguez-Nieva, M. Dresselhaus, T. Palacios, and J. Kong, Nano Letters 12, 161 (2012).

[13] C. R. Dean, A. F. Young, I. Meric, C. Lee, L. Wang, S. Sorgenfrei, K. Watanabe, T. Taniguchi, P. Kim, K. L. Shepard, and J. Hone, Nature Nanotech 5, 722 (2010).

[14] J. C. Meyer, A. Chuvilin, G. Algara-Siller, J. Biskupek, and U. Kaiser, Nano Letters 9, 2683 (2009).

[15] M. Carrard, D. Emin, and L. Zuppiroli, Phys. Rev. B 51, 11270 (1995).

[16] D. Emin, Journal of Solid State Chemistry 179, 2791 (2006).

[17] J. C. Lund, F. Olschner, F. Ahmed, and K. S. Shah, MRS Proc. 162 (1989) 10.1557/proc-162-601.

[18] T. P. Viles, B. A. Brunett, H. Yoon, J. C. Lund, H. Hermon, D. Buchenauer, K. McCarty, M. Clifft, D. Dibble, and R. B. James, MRS Proc. 487 (1997) 10.1557/proc-487-585.

[19] Y. Y. Platonov, L. Gomez, and D. Broadway, in X-ray mirrors, crystals, and multilayers II, edited by A. K. Freund, A. T. Macrander, T. Ishikawa, and J. L. Wood, (Dec. 2002).

[20] T. Tsarfati, R. van de Kruijs, E. Zoethout, E. Louis, and F. Bijkerk, Thin Solid Films 518, 1365 (2009).

[21] I. A. Makhotkin, E. Zoethout, R. van de Kruijs, S. N. Yakunin, E. Louis, A. M. Yakunin, V. Banine, S. Müllender, and F. Bijkerk, Opt. Express 21, 29894 (2013).

[22] V. Medvedev, A. Yakshin, R. van de Kruijs, and F. Bijkerk, Optical Materials Express 5, 1450 (2015).

[23] B. Padavala, C. D. Frye, X. Wang, Z. Ding, R. Chen, M. Dudley, B. Raghothamachar, P. Lu, B. N. Flanders, and J. H. Edgar, Crystal Growth \& Design 16, 981 (2016).

[24] B. Padavala, C. Frye, X. Wang, B. Raghothamachar, and J. Edgar, Journal of Crystal Growth (2016) 10.1016/j.jcrysgro.2016.05.031.

[25] J. A. Bearden, and A. F. Burr, Reviews of Modern Physics 39, 125 (1967).

[26] E. Schrödinger, Phys. Rev. 28, 1049 (1926).

[27] E. Schrödinger, Ann. Phys. 384, 489 (1926).

[28] E. Schrödinger, Ann. Phys. 385, 437 (1926).

[29] L. H. Thomas, Math. Proc. Camb. Phil. Soc. 23, 542 (1927).

[30] R. O. Jones, Reviews of Modern Physics 87, 897 (2015). 
[31] P. Hohenberg, and W. Kohn, Phys. Rev. 136, B864 (1964).

[32] W. Kohn, and L. J. Sham, Phys. Rev. 140, A1133 (1965).

[33] K. Burke, The Journal of Chemical Physics 136, 150901 (2012).

[34] A. D. Becke, The Journal of Chemical Physics 140, 18A301 (2014).

[35] R. V. Noorden, B. Maher, and R. Nuzzo, Nature 514, 550 (2014).

[36] D. Prendergast, and G. Galli, Phys. Rev. Lett. 96, 215502 (2006).

[37] J.-J. Velasco-Velez, C. H. Wu, T. A. Pascal, L. F. Wan, J. Guo, D. Prendergast, and M. Salmeron, Science 346, 831 (2014).

[38] A. M. Duffin, C. P. Schwartz, A. H. England, J. S. Uejio, D. Prendergast, and R. J. Saykally, The Journal of Chemical Physics 134, 154503 (2011).

[39] A. H. England, A. M. Duffin, C. P. Schwartz, J. S. Uejio, D. Prendergast, and R. J. Saykally, Chemical Physics Letters 514, 187 (2011).

[40] T. A. Pascal, C. D. Pemmaraju, and D. Prendergast, Phys. Chem. Chem. Phys. 17, 7743 (2015).

[41] W. S. Drisdell, R. Poloni, T. M. McDonald, J. R. Long, B. Smit, J. B. Neaton, D. Prendergast, and J. B. Kortright, J. Am. Chem. Soc. 135, 18183 (2013).

[42] J. Underwood, and E. Gullikson, Journal of Electron Spectroscopy and Related Phenomena 92, 265 (1998).

[43] E. M. Gullikson, S. Mrowka, and B. B. Kaufmann, Recent developments in euv reflectometry at the advanced light source, 2001.

[44] G. Kresse, and J. Hafner, Phys. Rev. B 47, 558 (1993).

[45] G. Kresse, and J. Hafner, Phys. Rev. B 49, 14251 (1994).

[46] G. Kresse, and J. Furthmüller, Computational Materials Science 6, 15 (1996).

[47] G. Kresse, and J. Furthmüller, Physical Review B 54, 11169 (1996).

[48] P. Giannozzi, S. Baroni, N. Bonini, M. Calandra, R. Car, C. Cavazzoni, D. Ceresoli, G. L. Chiarotti, M. Cococcioni, I. Dabo, A. D. Corso, S. de Gironcoli, S. Fabris, G. Fratesi, R. Gebauer, U. Gerstmann, C. Gougoussis, A. Kokalj, M. Lazzeri, L. Martin-Samos, N. Marzari, F. Mauri, R. Mazzarello, S. Paolini, A. Pasquarello, L. Paulatto, C. Sbraccia, S. Scandolo, G. Sclauzero, A. P. Seitsonen, A. Smogunov, P. Umari, and R. M. Wentzcovitch, Journal of Physics: Condensed Matter 21, 395502 (2009).

[49] E. L. Shirley, Phys. Rev. B 54, 16464 (1996).

[50] K. Watanabe, T. Taniguchi, and H. Kanda, Nat Mater 3, 404 (2004).

[51] K. K. Kim, A. Hsu, X. Jia, S. M. Kim, Y. Shi, M. Dresselhaus, T. Palacios, and J. Kong, ACS Nano 6, 8583 (2012).

[52] C. Jin, F. Lin, K. Suenaga, and S. Iijima, Phys. Rev. Lett. 102, 195505 (2009). 
[53] N. Alem, R. Erni, C. Kisielowski, M. D. Rossell, W. Gannett, and A. Zettl, Phys. Rev. B 80, 155425 (2009).

[54] K. Suenaga, H. Kobayashi, and M. Koshino, Phys. Rev. Lett. 108, 075501 (2012).

[55] O. Cretu, Y.-C. Lin, M. Koshino, L. H. G. Tizei, Z. Liu, and K. Suenaga, Phys. Rev. Lett. 114, 075502 (2015).

[56] S. Azevedo, J. R. Kaschny, C. M. C. de Castilho, and F. de Brito Mota, Nanotechnology 18, 495707 (2007).

[57] I. Jiménez, A. F. Jankowski, L. J. Terminello, D. G. J. Sutherland, J. A. Carlisle, G. L. Doll, W. M. Tong, D. K. Shuh, and F. J. Himpsel, Phys. Rev. B 55, 12025 (1997).

[58] M. Niibe, K. Miyamoto, T. Mitamura, and K. Mochiji, Journal of Vacuum Science \& Technology A 28, 1157 (2010).

[59] I. Caretti, and I. Jiménez, Journal of Applied Physics 110, 023511, (2011).

[60] K. Simonov, N. Vinogradov, M. Ng, A. Vinogradov, N. Mårtensson, and A. Preobrajenski, Surface Science 606, 564 (2012).

[61] P. E. Blöchl, Physical Review B 50, 17953 (1994).

[62] G. Kresse, and D. Joubert, Physical Review B 59, 1758 (1999).

[63] J. P. Perdew, K. Burke, and M. Ernzerhof, Phys. Rev. Lett. 77, 3865 (1996).

[64] A. Tkatchenko, and M. Scheffler, Phys. Rev. Lett. 102, 073005 (2009).

[65] H.-P. Komsa, T. T. Rantala, and A. Pasquarello, Physical Review B 86, 045112 (2012).

[66] C. G. V. de Walle, J. Appl. Phys. 95, 3851 (2004).

[67] A. H. England, A. M. Duffin, C. P. Schwartz, J. S. Uejio, D. Prendergast, and R. J. Saykally, Chemical Physics Letters 514, 187 (2011).

[68] W. Tang, E. Sanville, and G. Henkelman, Journal of Physics: Condensed Matter 21, 084204 (2009).

[69] Q. An, and W. A. Goddard, Chemistry of Materials 27, 2855 (2015).

[70] Q. An, K. M. Reddy, H. Dong, M.-W. Chen, A. R. Oganov, and W. A. Goddard, Nano Letters (2016) 10.1021/acs.nanolett.6b01204.

[71] G. Henkelman, B. P. Uberuaga, and H. Jónsson, The Journal of Chemical Physics 113, 9901 (2000).

[72] G. Henkelman, and H. Jónsson, The Journal of Chemical Physics 113, 9978 (2000).

[73] H. J. Monkhorst, and J. D. Pack, Phys. Rev. B 13, 5188 (1976).

[74] P. A. Schultz, Phys. Rev. Lett. 84, 1942 (2000). 
[75] M. Leslie, and N. J. Gillan, J. Phys. C: Solid State Phys. 18, 973 (1985).

[76] G. Makov, and M. C. Payne, Phys. Rev. B 51, 4014 (1995).

[77] S. Lany, and A. Zunger, Phys. Rev. B 78 (2008) 10.1103/physrevb.78.235104.

[78] C. Freysoldt, J. Neugebauer, and C. G. V. de Walle, Phys. Rev. Lett. 102 (2009) 10.1103/physrevlett.102.016402.

[79] T. L. Aselage, D. R. Tallant, and D. Emin, Phys. Rev. B 56, 3122 (1997).

[80] T. Stoto, N. Housseau, L. Zuppiroli, and B. Kryger, J. Appl. Phys. 68, 3198 (1990).

[81] T. Stoto, L. Zuppiroli, and J. Pelissier, Radiation Effects 90, 161 (1985).

[82] O. Cretu, Y.-C. Lin, and K. Suenaga, Micron 72, 21 (2015).

[83] Q. An, and W. A. Goddard, Chemistry of Materials 27, 2855 (2015).

[84] S. P. Huber, E. Gullikson, C. D. Frye, J. H. Edgar, R. W. E. van de Kruijs, F. Bijkerk, and D. Prendergast, Unpublished (2016).

[85] P. E. Blöchl, O. Jepsen, and O. K. Andersen, Phys. Rev. B 49, 16223 (1994).

[86] A. V. Krukau, O. A. Vydrov, A. F. Izmaylov, and G. E. Scuseria, The Journal of Chemical Physics 125, 224106 (2006).

[87] Y. Zhang, "Epitaxial growth of icosahedral boron arsenide on silicon carbide substrates: improved process conditions and electrical properties",

PhD thesis (Kansas State University, 2011).

[88] D. R. Armstrong, J. Bolland, and P. G. Perkins, Theoretica Chimica Acta 64, 501 (1984).

[89] D. Li, and W. Y. Ching, Phys. Rev. B 52, 17073 (1995).

[90] W. Hayami, and S. Otani, J. Phys. Chem. C 112, 2711 (2008).

[91] G. A. Slack, T. McNelly, and E. Taft, Journal of Physics and Chemistry of Solids 44, 1009 (1983).

[92] B. Padavala, C. Frye, Z. Ding, R. Chen, M. Dudley, B. Raghothamachar, N. Khan, and J. Edgar, Solid State Sciences 47, 55 (2015).

[93] G. Li, J. K. Abbott, J. D. Brasfield, P. Liu, A. Dale, G. Duscher, P. D. Rack, and C. S. Feigerle, Applied Surface Science 327, 7 (2015).

[94] Z. Jia, J. Zhu, C. Jiang, W. Shen, J. Han, and R. Chen, Applied Surface Science 258, 356 (2011).

[95] W. Liu, H. Tu, H. Yang, S. Zhang, L. Yan, C. Huo, and X. Su, Journal of Alloys and Compounds 538, 169 (2012).

[96] V. A. Mukhanov, P. S. Sokolov, Y. L. Godec, and V. L. Solozhenko, J. Superhard Mater. 35, 415 (2013). 
[97] V. A. Mukhanov, P. S. Sokolov, O. Brinza, D. Vrel, and V. L. Solozhenko, J. Superhard Mater. 36, 18 (2014).

[98] H. Sugimoto, M. Fujii, and K. Imakita, RSC Adv. 5, 8427 (2015).

[99] V. A. Mukhanov, D. Vrel, P. S. Sokolov, Y. L. Godec, and V. L. Solozhenko, Dalton Trans. (2016) 10.1039/c6dt00435k.

[100] A. López-Castillo, Int. J. Quantum Chem. 112, 3152 (2012).

[101] J. Yu, and W. Guo, Appl. Phys. Lett. 106, 043107 (2015).

[102] D. Çakır, D. Kecik, H. Sahin, E. Durgun, and F. M. Peeters, Phys. Chem. Chem. Phys. 17, 13013 (2015).

[103] J. Dong, H. Li, and L. Li, NPG Asia Materials 5, e56 (2013).

[104] M. Mirzaei, and M. Meskinfam, Solid State Sciences 13, 1926 (2011).

[105] Z. Sohbatzadeh, M. Roknabadi, N. Shahtahmasebi, and M. Behdani, Physica E: Low-dimensional Systems and Nanostructures 65, 61 (2015).

[106] S.-f. Wang, and X.-j. Wu, Chin. J. Chem. Phys. 28, 588 (2015).

[107] D. Vanderbilt, Phys. Rev. B 41, 7892 (1990).

[108] K. Laasonen, R. Car, C. Lee, and D. Vanderbilt, Phys. Rev. B 43, 6796 (1991).

[109] D. McCulloch, D. Lau, R. Nicholls, and J. Perkins, Micron 43, 43 (2012).

[110] D. S. Franzblau, Phys. Rev. B 44, 4925 (1991).

[111] A. B. Altman, C. D. Pemmaraju, C. Camp, J. Arnold, S. G. Minasian, D. Prendergast, D. K. Shuh, and T. Tyliszczak, J. Am. Chem. Soc. 137, 10304 (2015).

[112] W. S. Drisdell, R. Poloni, T. M. McDonald, T. A. Pascal, L. F. Wan, C. D. Pemmaraju, B. Vlaisavljevich, S. O. Odoh, J. B. Neaton, J. R. Long, D. Prendergast, and J. B. Kortright, Phys. Chem. Chem. Phys. 17, 21448 (2015).

[113] T. A. Pascal, C. D. Pemmaraju, and D. Prendergast, Phys. Chem. Chem. Phys. 17, 7743 (2015).

[114] S. N. Patel, G. M. Su, C. Luo, M. Wang, L. A. Perez, D. A. Fischer, D. Prendergast, G. C. Bazan, A. J. Heeger, M. L. Chabinyc, and E. J. Kramer, Macromolecules 48, 6606 (2015).

[115] C. P. Schwartz, and D. Prendergast, The Journal of Chemical Physics 143, 111102 (2015).

[116] C. Schwartz, D. Nordlund, T.-C. Weng, D. Sokaras, L. Mansfield, A. S. Krishnapriyan, K. Ramanathan, K. E. Hurst, D. Prendergast, and S. T. Christensen, Solar Energy Materials and Solar Cells 149, 275 (2016). 
[117] J. W. Smith, R. K. Lam, O. Shih, A. M. Rizzuto, D. Prendergast, and R. J. Saykally, The Journal of Chemical Physics 143, 084503 (2015).

[118] L. F. Wan, J. Wright, B. R. Perdue, T. T. Fister, S. Kim, C. A. Apblett, and D. Prendergast, Phys. Chem. Chem. Phys. 18, 17326 (2016). 



\section{ACKNOWLEDGMENTS}

First and foremost, I thank David, for bravely taking on the task of being my daily supervisor for the past three years. Coincidence had it that, barely a week after our first conversation on the phone, you happened to be in Utrecht for a conference, which gave me the opportunity to "corner" you in a restaurant and convince you to take me on as your student. I can not help but wonder how different this work would have turned out, had this string of unplanned events not taken place. You truly have taught me a lot during my stay at LBNL, not just from a scientific perspective, but also on a personal level; how to deal with those moments of self-doubt and sense of inadequateness, which are arguably inevitable when collaborating with truely ambitious and intelligent people. It has also been thanks to your endless optimism that, no matter how stuck I felt in the research, I would always leave our weekly discussions with a renewed sense of direction and motivation.

Of course this entire project has been made possible to begin with by Fred, to whom as my promotor I am very grateful. Together with Robbert as my supervisors in the Netherlands, you have made sure that the overall planning of the work went smoothly. I can only image that being a supervisor to a student that is half way across the world for most of the time, is challenging to say the least. Despite this difficulty, you have always helped me greatly during our discussions, whether over the phone or the few times in person whenever I was in Twente, in keeping the bigger picture and thinking of possible directions to take the research in.

To Eric, both my experimental supervisor and partner in crime on the bike with the Berkeley Bicycle Club, I extend my gratitude. I still think that our chats during the usual wednesday morning bike rides going up Tunnel Road, are a particularly efficient and enjoyable way of discussing a project's progress. With your expertise you have really helped the experimental part of this work and I am grateful for the many slots of beam time that you have made available to me. I also want to thank Julia for lending an expert hand during all those beam time experiments.

To my colleagues at the theory group of the Molecular Foundry; Tod, Das, Yufeng, Sabrina, Артём, Joyjit and Kristi, you have really helped me a great deal in getting up to speed in the complex and beautiful world of computational materials science. With your various specific fields of expertise, you have truly made a huge difference in the progress of my research, as often any problem could be solved by a quick visit to your office, which made for a really motivating environment. Additionally, and not wholly unimportantly, the group has always felt as a really friendly, supportive and collaborative group. A big thanks to all of you.

Then to Stein, Gustav, a.k.a. Double G, the one and only Batty Boy (the list goes on). I could never have imagined, sitting on the lawn of het Minnaert in 2005, that eleven years later, we would both still be attempting to do physics. Many unforgettable 
moments since then, such as impromptu arm wrestle brawls, the formulation of sharp ansatzes, the distributions (diestroeboeties) and police corollaries of Kuznetsov, the jungle pants and fanny pack of a certain cosmologist and countless exercises made in a futile attempt to come to grips with the fundamentally incomprehensive theory of quantum mechanics, have all become invaluable memories. Our road trip of the United States this year, cemented the realization that you are a truly exceptional friend on an intellectual, spiritual and musical level. Monorail.

Dear Игорь, unfortunately the considerable distance between Berkeley and Twente, has prevented us from collaborating as intensively as we might have wanted, however I still owe you many thanks. Working closely with you during my master's research has prepared me well for the challenges that lay ahead of me in this research project. I hope we will get the chance to collaborate once again in the future.

Слава, as the one who introduced me to boron phosphide, I owe you an enormous thank you, as more than half of this work would not have come to a realization, had you not brought it to my attention. Also, it has been a great pleasure to directly collaborate with you, despite not being able to do it in person like when we were still both working at Rijnhuizen. It has very nearly been as pleasing as that one time you nearly died laughing after I tried to swear in Russian.

Thanks to all my colleagues of the XUV-TNW group, with a special shout out to Eric, Marko, Дмитрий and Андрей, who have helped me with coordinating experiments in Twente.

My dearest "huisbutjes" Hilde, Frederik, Roderik, Jochum and their significant others, Judith, Floor, Lieveke en Myrthe can not be omitted from this giving of thanks. Despite me having to leave our beloved Normandie 170 and therewith your company, to travel halfway across the world, you have always remained a valuable source of motivation and great listeners whenever I needed to vent. Rather counterintuitive given how annoyed you get with my quotes. Not really counterintuitive, I just needed that slip that word in for the challenge.

Then to conclude with the people that have been there from the start. Pââhpt, moederlief and snoer, I have never ever had to doubt the fact that you were proud of me, which is arguably the biggest motivator a person can have. I love you to bits. 


\section{NEDERLANDSE SAMENVATTING}

Boor, het vijfde element in het periodiek stelsel der elementen, vormt de rode draad door dit proefschrift. Dit element heeft een elektronen configuratie waarbij de buitenste schil drie elektronen bevat, wat direct ten grondslag ligt aan het brede scala aan chemische verbindingen en kristalstructuren die boor kan vormen met andere elementen. De verschillende verbindingen waarin boor voorkomt spreiden dientengevolge ook een grote variatie in elektronische en structurele eigenschappen tentoon. Zo vormt het hexagonale boor nitride $(h-\mathrm{BN})$ met zijn honingraatstructuur een goede insulator, maar is het rhombohedrische boor phosphide $\left(\mathrm{B}_{12} \mathrm{P}_{2}\right)$ een halfgeleider. Dit zijn slechts twee voorbeelden van boorverbindingen die bijzonder interessante eigenschappen hebben voor verschillende toepassingen. Echter zijn er nog open vragen wat betreft de precieze structuur van deze kristallen en specifiek hoe kristal defecten ontstaan en deze de wenselijke eigenschappen beinvloeden. In dit werk is bestudeerd hoe x-ray absorptie near-edge spectroscopie (XANES) inzicht kan geven in de origine en kenmerken van deze boor kristal structuren en de mogelijke defecten daarin.

X-ray absorptie spectroscopie berust op de eigenschap dat elektronen in atomen zich ordenen in zogeheten elektronenschillen en dat afhankelijk van de schil waarin het elektron zich bevindt, alleen fotonen met een karakteristieke energie geabsorbeerd kunnen worden. Deze karakteristieke energie is kenmerkend voor de elektronenschil, maar ook voor het element waartoe het atoom behoort. Dientengevolge is XANES een element gevoelige techniek die in verbindingen met meerdere elementen, selectief met één element kan interacteren. Bovendien worden de karakteristieke energieen waarvoor de elektronen gevoelig zijn, ook beinvloed door de directe omgeving van het atoom waartoe ze behoren. Dat wil zeggen, afhankelijk van de chemie en structuur die het atoom met zijn directe buur atomen vormt, verandert het karakteristieke x-ray absorptie spectrum. Omgekeerd kan een verandering in het spectrum dus ook herleid worden naar een verandering in chemie en structuur.

De experimentele x-ray spectroscopie metingen die zijn verricht met de bundellijn 6.3.2 van de Advanced Light Source aan de verscheidene boorverbingen bevatten in theorie dus informatie over de structurele en chemische eigenschappen van de verbindingen op een atomaire schaal. Om deze informatie te extraheren, moet een structureel model geconstrueerd worden waarvoor de x-ray absorptie spectroscopie wordt gesimuleerd. Het simuleren van XANES is in dit werk gedaan met behulp van density functional theory (DFT), een methode gegrond in de quantum mechanica, die stelt dat voor de potentiaal van een gegeven atoom structuur, de elektronen dichtheid distributie uniek bepaald is. Met deze stelling kan de tijdsonafhankelijke Schrödinger vergelijking opgelost worden door deze unieke elektronen dichtheid te bepalen, wat de golf vergelijking van het systeem oplevert en daarmee alle benodigde informatie verschaft, om welke eigenschap van het systeem dan ook te berekenen. Een voorbeeld daarvan is de x-ray 
absorptie spectroscopie van het systeem in kwestie, de eigenschap van interesse in dit proefschrift.

Met deze DFT methode om experimenteel verkregen x-ray absorptie spectroscopie te modelleren, zijn verschillende open vragen betreffende de structurele eigenschappen van boorverbindingen beantwoord. Zo is in de literatuur veelvuldig het ontstaan van driehoeksvormige gaten in $h$-BN geobserveerd met behulp van een elektronen microscoop, alsmede drie bijbehorende karateristieke absorptie lijnen in de x-ray spectroscopie. Dit werk laat zien dat de kenmerkende absorptie lijnen veroorzaakt worden door zuurstofatomen die zijn opgenomen in het hexagonale kristalrooster. De verschillende coordinatie voorkeur van zuurstof ten opzichte van stikstof verklaart ook waarom de gaten die in het materiaal gevormd worden driehoeks symmetrie hebben, waarbij de rand gevormd wordt door de zuurstof atomen die de gaten stabiliseren.

Experimenten uit de jaren negentig aan $\mathrm{B}_{12} \mathrm{P}_{2}$ suggereerden dat het materiaal een zelfreparerende eigenschap bezit, nadat opnamen met een elektronen microscoop een vrijwel onbeschadigd materiaal onthulden, ondanks dat het materiaal direct daarvoor met hoog energetische deeltjes was gebombardeerd. De oorsprong van de zelf-repararende kwaliteit was tot op heden niet bekend, maar de simulaties gepresenteerd in dit proefschrift laten zien dat $\mathrm{B}_{12} \mathrm{P}_{2}$ inderdaad "self-healing" is en dat dit mede is dankzij de robuustheid van het boor icosahedron, het bouwblok waaruit de $\mathrm{B}_{12} \mathrm{P}_{2}$ kristalstructuur bestaat. Nudged elastic band (NEB) simulaties laten zien dat gaten die onstaan, door de verplaatsing van boor atomen uit het kristalrooster, weer opgevuld worden in een door de boor atomen zelf gecoordineerde en collaboratieve beweging. De energie barriere voor deze schade herstellende processen zijn bijzonder laag, waardoor het de ogenschijn wekt dat het schadeherstel uit zichzelf voorkomt. X-ray absorptie spectroscopie experimenten, uitgevoerd in dit werk, hebben bevestigd dat $\mathrm{B}_{12} \mathrm{P}_{2}$ samples inderdaad weinig boor defecten bevatten, een direct gevolg van de zelf-helende eigenschap van dit materiaal.

De resultaten gepresenteerd in dit werk, laten zien dat x-ray absorptie spectroscopie een krachtige methode is om de structuur van boorverbindingen op atomaire schaal te beschrijven. Een verbeterd inzicht in de structurele eigenschappen van deze materialen levert vervolgens de mogelijkheid om hun geschiktheid voor de respectievelijke toepassingen ook te verbeteren. Een bijzonder waardevol aspect van het verbeteren van de simulatie van x-ray absorptie spectroscopie, door middel van een "first-principles" methode zoals DFT, is dat als deze betrouwbaar genoeg wordt, materiaal eigenschappen ook voorspeld kunnen worden, alvorens experimenten zijn uitgevoerd. Dit opent de weg naar het voorspellen van het bestaan en het ontwikkelen van nog niet eerder ontdekte materialen met specifieke waardevolle eigenschappen. 
(1)

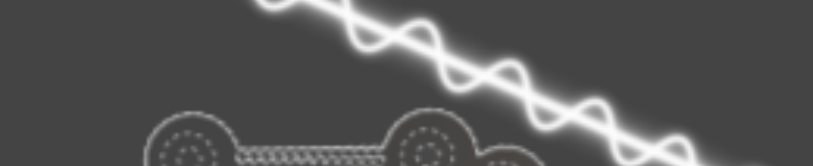

23

Ditis

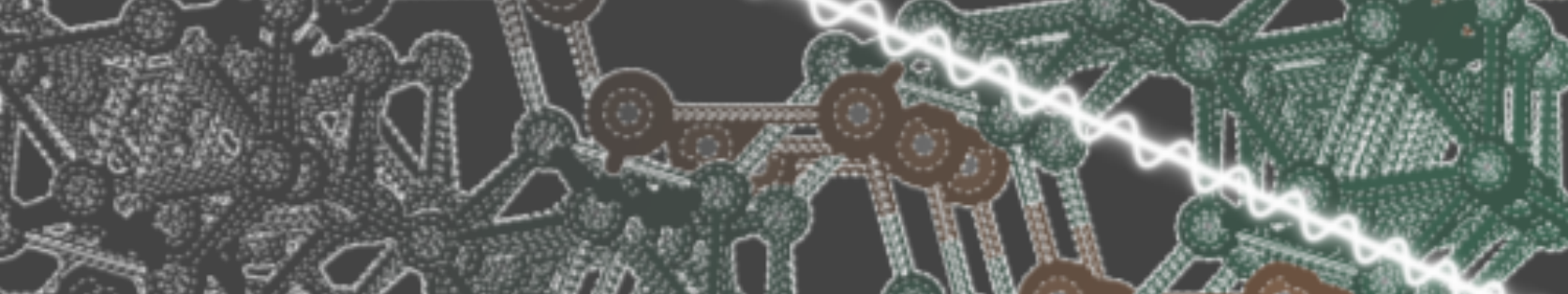
201210 PIn

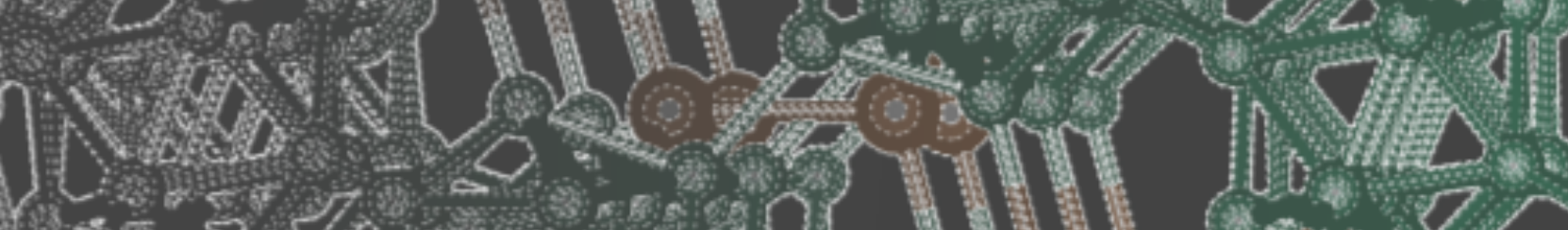

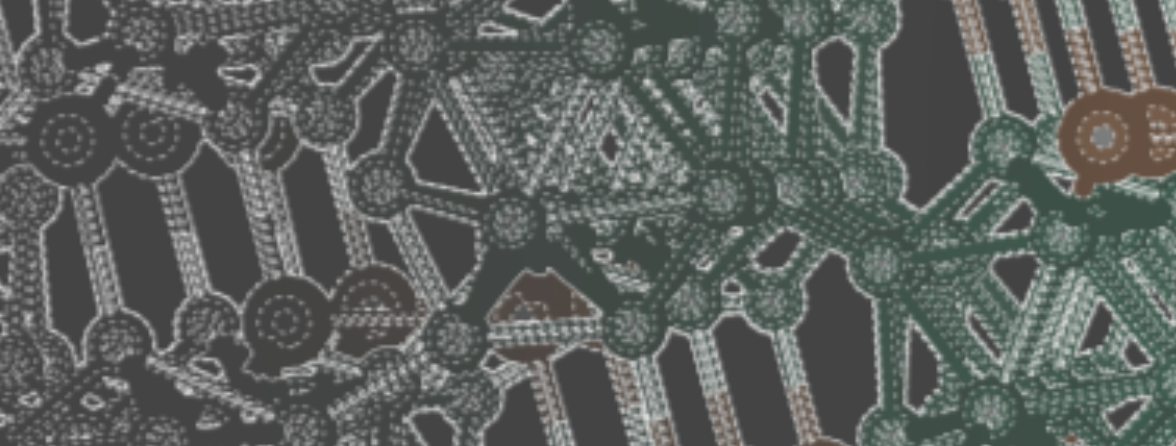

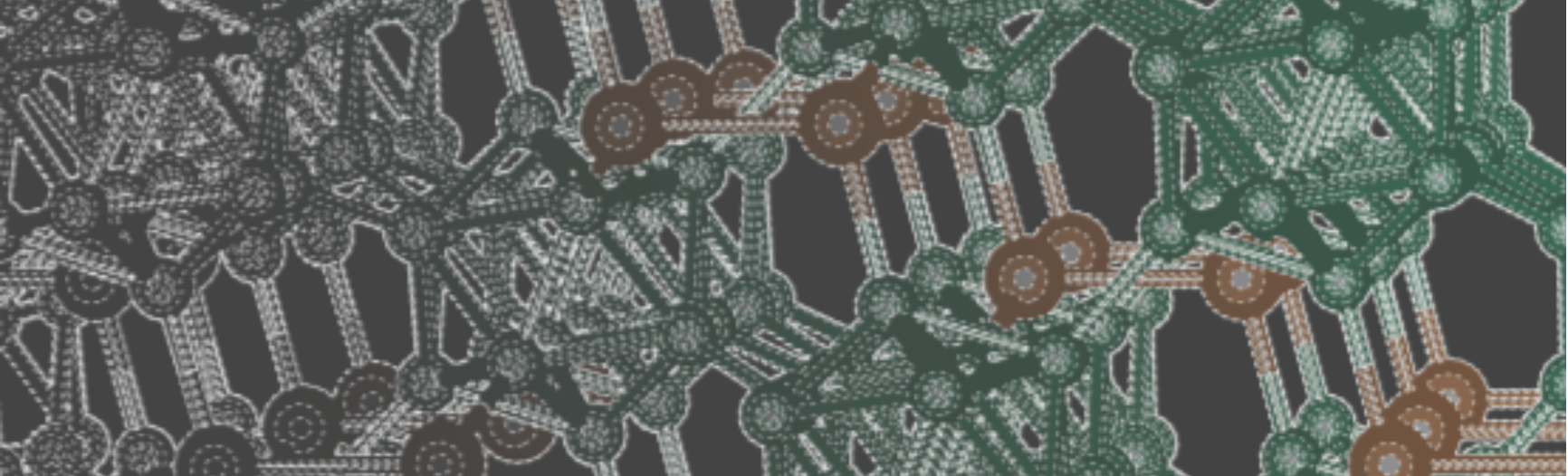

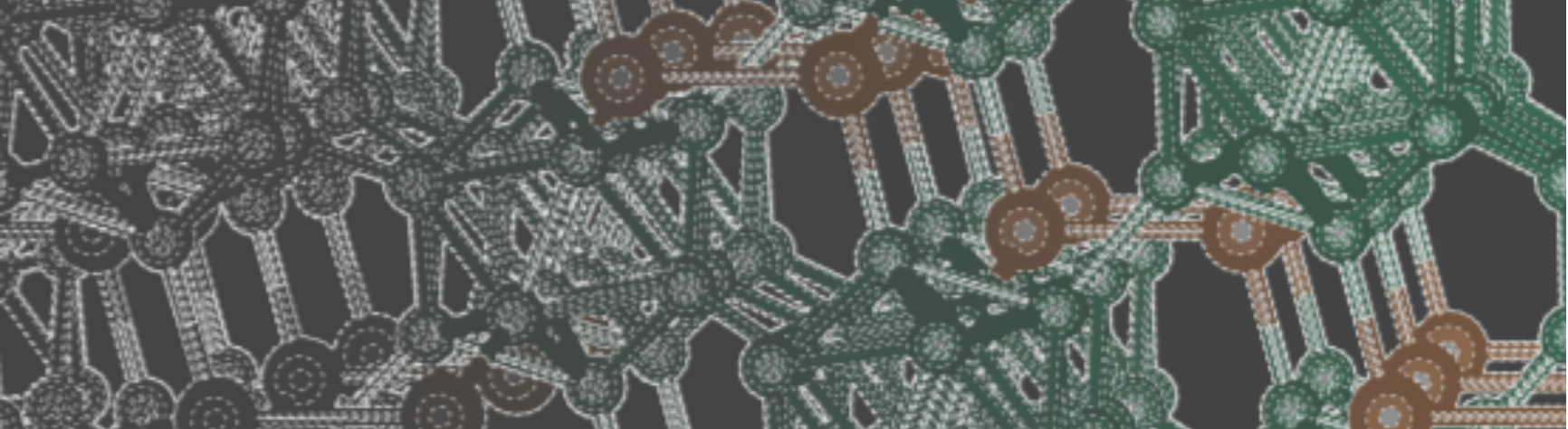

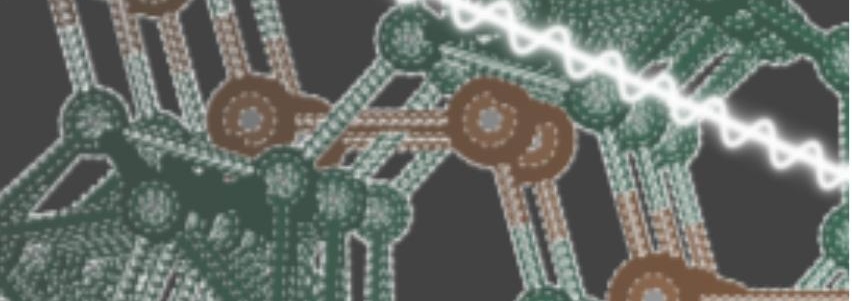

